$f^{5}+x^{2}+2 x$

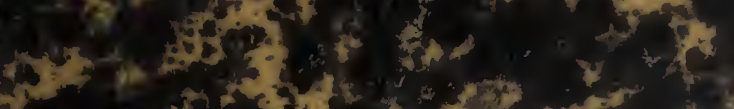

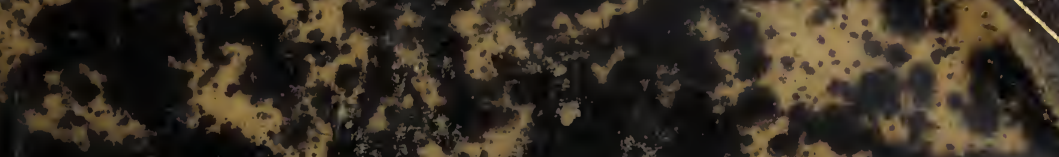

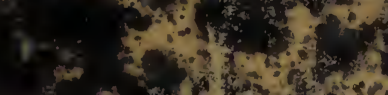

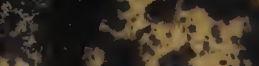

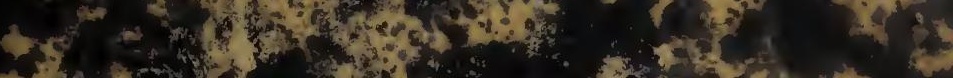

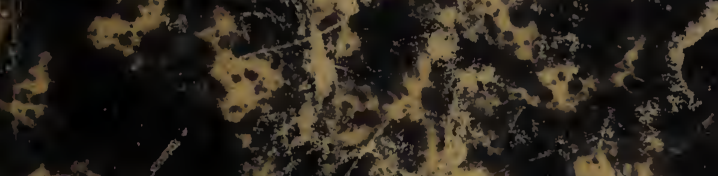

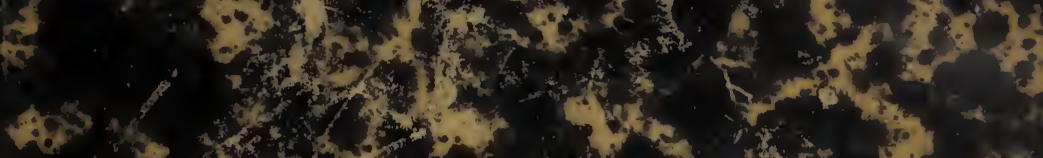

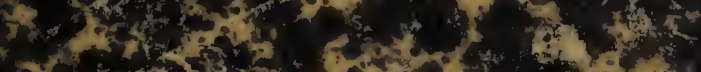

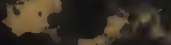

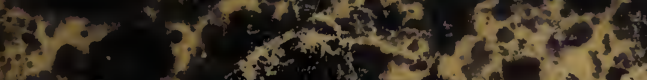

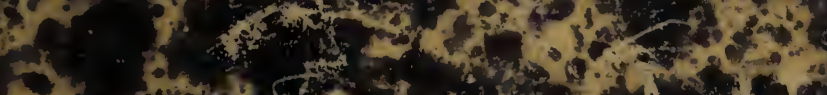

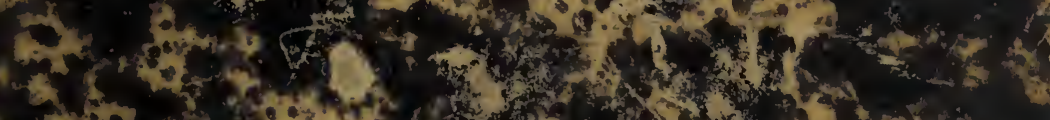

7.

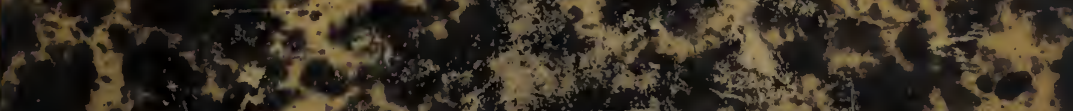

2.

97 . (20)

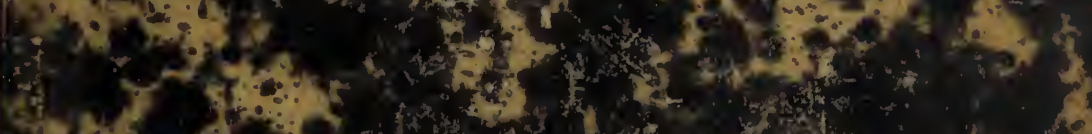

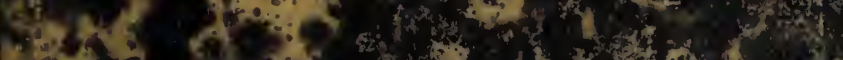

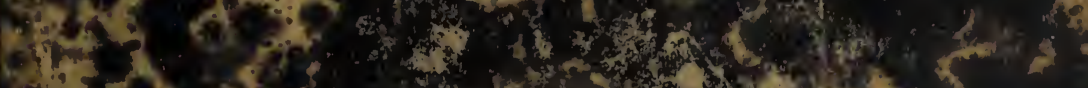

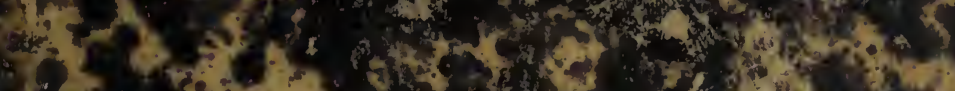

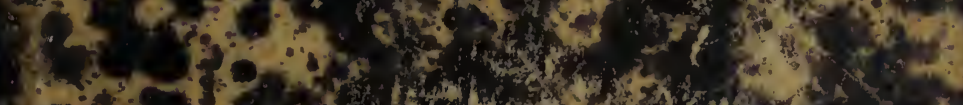

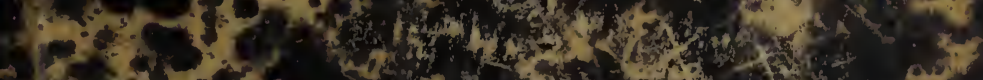

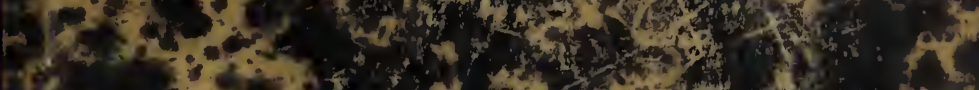

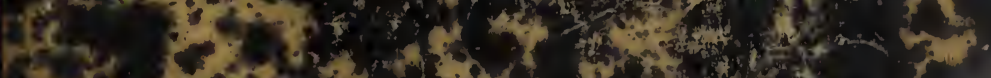
3.t. 3.

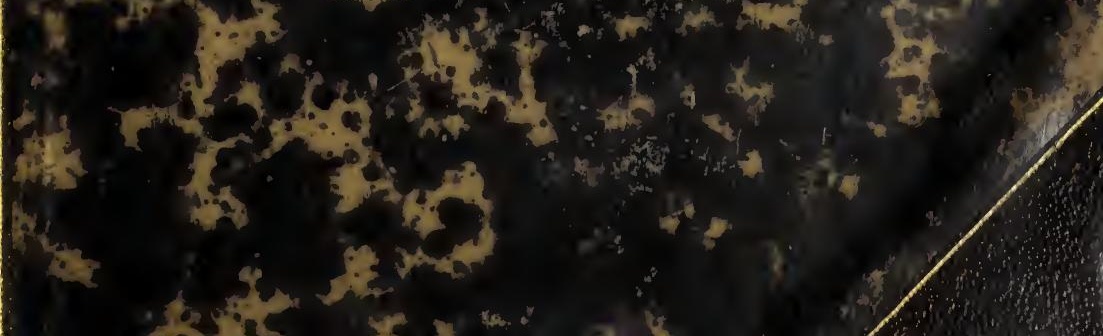


- REMTTE STORAGE
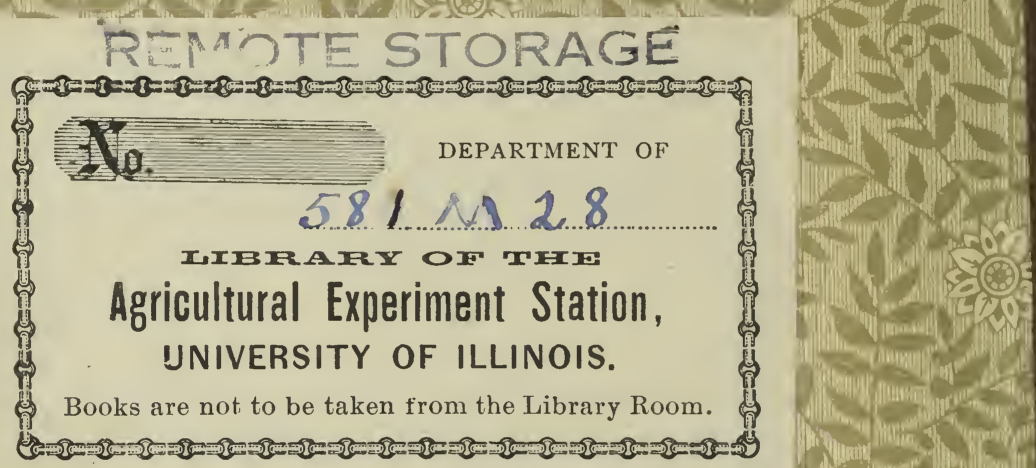

Von
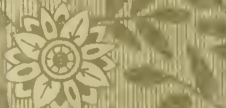

(19) 


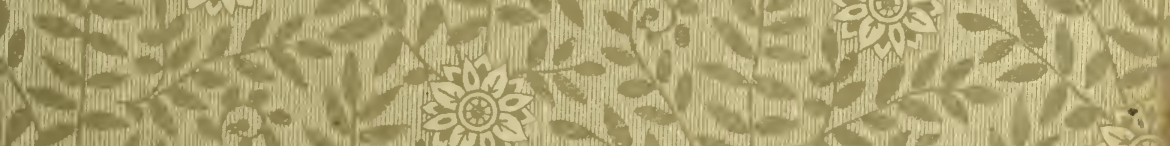

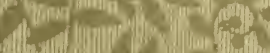

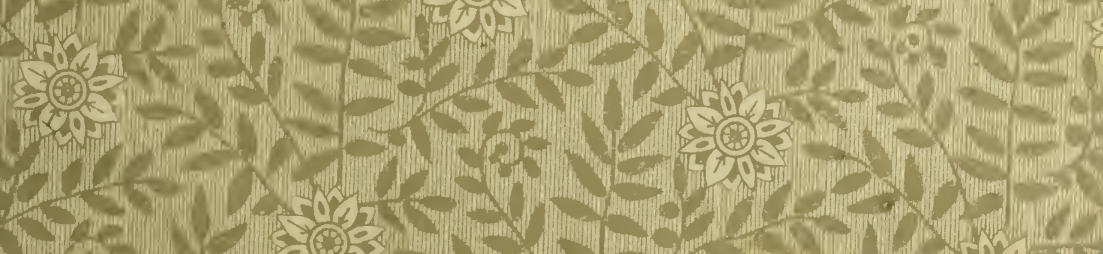


Digitized by the Internet Archive in 2016 with funding from

University of Illinois Urbana-Champaign Alternates

https://archive.org/details/botaniqueanatomi00mais 




\section{NOUVEAU COURS D'HISTOIRE NATURELLE}

BOTANIQUE 
TYPOGRAPHIE

EDMOND MON NOYER

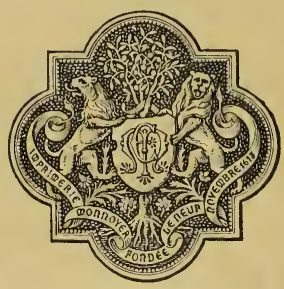

AU MANS (SARthe) 


\section{NOULEAU COURS D'TIISTOIRE MATURELLI}

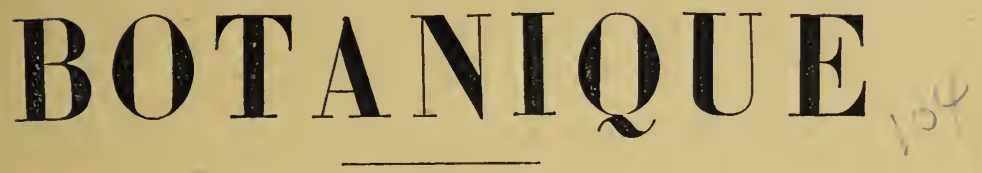

\section{A N A T O M I E}

\section{ET PHYSIOLOGIE VÉGÉTALES}

OUVRAGE RÉDIGÉ CONFORMÉMENT AUX PROGRAMMES OFFICIELS

DU 2 JANVIER 1883

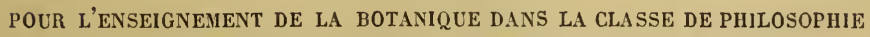
ET L'EXAMEN DU BACCALAURÉAT ĖS LETTRES

\section{PAUL MAISONNEUVE}

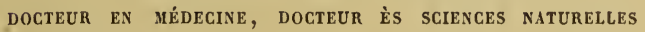
PROFESSEUR a La FaCULTÉ LIBRE DES SCIENCES D'ANGERS

Natura non facit saltus.

Tout s'enchaine dans la nature.

3. Lixxḱ.

Ourrage orné de 154 figures

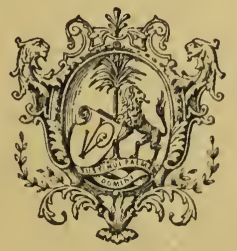

\section{PARIS}

VIGTOR PALMÉ, LIBRAIRE-ÉDITEUR

76, rue des Saints-Pères, 76

BRUXELLES

SOCIÉTÉ BELGE DE LIBRAIRIE

12, rue des Paroissiens, 12
GENEेVE

H. TREMBLEY, ÉDITEUR

4, rue Corraterie, 4 


$$
\begin{aligned}
& 580 \\
& 128 \\
& 20
\end{aligned}
$$

REMOTE STORAGE a.e.

$$
\text { t.e. }
$$

$$
1 . .
$$




\title{
PROGRAMME OFFIGIEL
}

\section{IUU COULIS D’ANATONIE ET DE P.IISIOLOGIE VÉGÉTALES \\ POUR LA CLASSE DE PHILOSOPHIE}

\begin{abstract}
Arrêté ministériel du 22 janvier 1885.
\end{abstract}

\section{Anatomie et Physiologie végétales.}

Pages.

Caractères généraux des végétaux.................. xı. x.

Cellule végétale, formation des tissus. - Principaux tissus... 1

I. - Nutrition (Étude spéciale d'une plante phanérogame). Tige : croissance et fonctions de la tige.............. $4 \mathbb{4}$

Racine. - Radicelles. - Croissance et fonctions de la racine. 82

Feuille: structure, croissance et fonctions............. 94

Nutrition en général : plantes à chlorophylle, plantes sans chlorophylle. - Aliments. - Réserves nutritives. - Respiration. 106

II. - Reproduction (Étude spéciale d'une plante phanérogame).

Fleur : enveloppes florales; structure de l'étamine; anthère ; pollen; structure des carpelles; ovule........... 149

Fécondation et développement................... 188

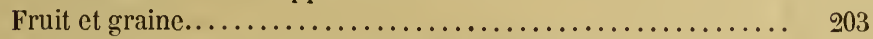

Germination : phénomènes qui l'accompagnent........... 213

Cryptogames : reproduction et formes alternantes. -

parasitisme............................ 220

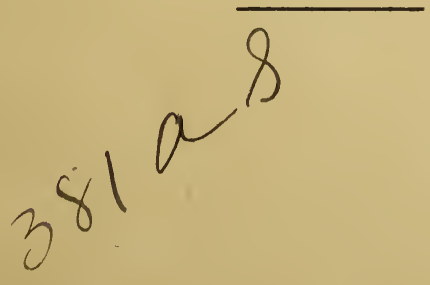





\section{PRÉFAGE}

Nous n'en sommes plus au temps de Tournefort, où les seigneurs et les dames de la cour elles-mêmes, entrainés par l'enseignement séduisant de ce grand naturaliste, allaient dans les bois des environs de Paris étudier la Botanique, devenue en quelque sorte une science à la mode. Pourquoi cette aimable science est-elle si négligée de nos jours et ne trouvet-elle plus guère d'adeptes que parmi ceux qui ont quelque prétention au titre de savant ou qui ont besoin de la connaître par devoir professionnel? Sans vouloir sonder la question ou remonter aux causes d'un semblable état de choses, peut-être me sera-t-il permis, tout au moins, d'émettre l'avis que l'abondance et la variété des notions qui sont imposécs aux jeunes.gens n'y sont pas étrangères, de sorte que le temps leur suffit à peine pour acquérir une connaissance suffisante des matières dont sont 
chargés leurs programmes, afin d'obtenir, au plus tôt, le diplôme désiré de bachelier, puis de s'enfermer, du moins ceux qui ont le goût du travail, dans les écoles spéciales ou se livrer aux études ardues de quelque carrière laborieuse dont la préparation absorbe tous leurs instants.

Et pourtant, l'étude de l'Histoire naturelle n'est-elle pas des plus propres à reposer l'esprit trop tendu par des travaux souvent exagérés? La Botanique notamment, l'étude des plantes, qui est inséparable de promenades au milieu des champs et des bois, n'est-elle pas la meilleure et la plus saine des distractions que l'on puisse proposer à un jeune homme? Cette intéressante étude, conduite avec intelligence, exerce le corps et l'esprit, tout en les fortifiant et reposant l'un et l'autre à la fois.

Mais, pour la plupart, nous passons à côté des merveilles que la nature généreuse étale à nos yeux, sans y prêter le plus souvent la moindre attention, sans daigner nous baisser pour contempler l'une de ces beautés. Cependant, c'est une phrase comme stéréotypée, que tout dans la nature est merveilleux, que tout a été fait avec nombre, poids et mesure. Mais, grand Dieu! qu'ils sont nombreux ceux qui croient à cette vérité sur la foi des autres, et se trouveraient bien embarrassés d'en faire la démonstration, alors qu'il leur serait facile et si doux de la vérifier par eux-mêmes.

On ne saurait donc trop encourager les jeunes gens à consacrer quelques-uns de leurs loisirs à 
l'étude des choses de la nature. Quelle plus charmante occupation, par exemple, que de considérer une fleur, se rendre compte de sa forme, en examiner attentivement l'une après l'autre, chaque partie, le calice, aux folioles assez épaisses, recouvrant à demi les délicats pétales de la corolle aux couleurs vives et au délicieux parfum qui attirent les insectes, êtres inconscients du rôle souvent considérable qu'ils vont jouer dans la multiplication de la plante, en transportant au moyen de quelque partie de leur corps la fine poussière fécondante qui sort de petites capsules, les anthères, et qui mise au contact d'autres petits corps que la fleur recèle dans son sein et que protègent tous les organes extérieurs artistement disposés autour d'eux, deviendra la graine, en qui se trouve renfermée la promesse d'une génération nouvelle?

Maintenant, si poursuivant cette étude, au lieu de se contenter de considérer la forme et les relations superficielles de ces organes, le jeune homme aidé d'un instrument grossissant pénètre plus avant dans la connaissance de l'organisation, étudie la structure intime, la délicatesse des tissus, les cellules aux parois si fragiles qui, agencées de différentes façons, forment la charpente :de cet élégant édifice, pourra-t-il ne pas être saisi d'admiration en présence de tant de merveilles accumulées dans de si petits objets?

Que sera-ce encore, si poussant plus loin ses recherches, il s'efforce de surprendre les manifesta- 
tions vitales, les fonctions de cet être auquel la Providence a accordé une sorte de vie, s'il voit les mouvements qui s'accomplissent dans les cellules, la circulation, dans toutes les parties de l'organisme, de certains liquides, sorte de sang que des vaisseaux sont chargés de distribuer; s'il surprend les procédés employés par la Plante pour se nourrir, en empruntant au règne minéral les aliments dont elle a besoin, la voie par laquelle elle les introduit dans son organisme, les métamorphoses physiques et chimiques qu'elle leur fait subir dans l'intimité de ses tissus; comment un petit nombre de principes minéraux, carbone, hydrogène, oxygène, azote, se transforment en cette variété infinie de composés organiques si complexes que renferme la Plante, tels que fécule, sucre, matières grasses, substances albuminoïdes; comment elle amasse dans certains organes des provisions ou réserves nutritives, pour les utiliser à un moment donné; les substances variées qu'elle sécrète et qui, renfermées dans de petites cavités closes ou dans certains canaux, sont souvent recherchées par l'homme pour servir à son alimentation ou à des usages médicaux ou industriels?

Quel que soit le point de vue auquel on se place pour étudier la Botanique, on est sûr d'y trouver un grand charme et souvent une réelle utilité pratique.

Pénétré des idées qui viennent d'être exposées, nous avons composé cet ouvrage où se trouvent réunies les notions élémentaires de la Botanique, avec le désir qu'il ne soit pas, pour tous, seulement 
un Manuel destiné à répondre aux exigences du Baccalauréat, mais, au moins pour quelques-uns, une initiation à la plus aimable des sciences, qu'il en développe le goût et excite en eux le désir de pénétrer plus avant dans sa connaissance.

Le plan que nous avons suivi est des plus naturels, et celui qui paraît le mieux convenir aux ouvrages didactiques qui ont pour objet les sciences expérimentales. Procédant du simple au composé, nous considérons d'abord les parties élémentaires des Végétaux, qui tous ont pour point de départ la Cellule, dont nous étudions la composition et les transformations qu'elle peut subir. Passant ensuite à l'étude des substances contenues dans les cellules végétales, nous passons en revue les principales, aussi bien celles qui jouent un rôle dans la nutrition de la Plante et doivent être entraînées, à un moment donné, dans le tourbillon vital, que celles qui constituent des matières d'excrétion et qui ne paraissent plus devoir jouer aucun rôle dans la vie de l'organisme. De l'étude des éléments anatomiques et des tissus qu'ils forment, passant aux organes, nous examinons d'abord ceux qui concourent à la nutrition, à savoir la Tige, la Racine, les Feuilles, pour aborder, aussitôt après, l'étude de leurs fonctions. On remarquera que celles-ci portent les mêmes noms que ceux employés à propos des Animaux; c'est qu'en effet, comme on va le dire dans les pages suivantes, les procédés mis en œuvre et les résultats obtenus sont très comparables à ceux que nous 
offrent ces derniers. Passant alors aux organes qui servent à la reproduction de la Plante, c'est-à-dire à la Fleur, nous en indiquons brièvement la composition, la structure, afin de pouvoir suivre les phénomènes intimes qui précèdent ou qui accompagnent la formation du Fruit et de la Graine. L'exposé de ces mêmes phénomènes considérés chez les organismes végétaux les plus inférieurs termine cette étude en nous réservant de véritables surprises par leur singulière et merveilleuse complexité. La question si importante du Parasitisme chez les Végétaux sera ensuite rapidement traitée. Enfin, quelques considérations sur les mouvements partiels ou d'ensemble offerts par les végétaux complèteront l'ouvrage.

Ainsi aurons-nous passé en revue les traits principaux de la vie des Plantes, dont les notions d'anatomie acquises chemin faisant faciliteront singulièrement l'intelligence.

Angers, 6 janvier 1886. 


\section{INTRODUCTION}

\section{Caracteires gétéradu des véGÉTaux}

Les Plantes peuvent être étudiées à différents points de vue ; ainsi on peut considérer leur organisation, leur structure, autrement dit, leur Anatomie, qui se partage en Organographie, Organogénie, Histologie ou anatomie proprement dite, selon qu'on se borne à l'examen de la configuration et des caractères extérieurs des organes, ou qu'on observe le mode d'apparition de ceux-ci, ou bien, enfin, qu'il s'agit de la connaissance des parties élémentaires ou éléments anatomiques qui entrent dans leur constitution.

On peut encore se placer à un autre point de vue, celui des fonctions' accomplies par les Végétaux, ou ce qu'on appelle leur Physiologie, c'est-à-dire, examiner comment ils se nourrissent, s'accroissent, se reproduisent, etc... (1).

Or, ces deux parties qui, au premier abord, paraissent si différentes, sont intimement liées l'une à l'autre, de sorte

(1) Nous laissons volontarrement de côté un troısième point de vue, qui a également une grande importance en Botanique, à savoir la distribution méthodique des plantes et leur groupement en espèces, genres, familles, etc., auquel répond le programme de la Classe de Cinquième. (Voyez Nolions elémentaires de Zoologie, Botanique, Géologie, rédigées conformément au programme officiel, pour les Classes de Sixième, Cinquième, Quatrième, par le docteur P. MAISONNEUVE.) 
que leur étude ne peut guère être séparée. Comment, en effet, serait-il possible d'observer et de suivre les phénomènes dont les Plantes sont le siège, si l'on n'a pas, au préalable, pris connaissance de la disposition, de la structure des parties qui entrent en jeu? Pour observer comment fonctionne une machine, ne faut-il pas d'abord en connaître les rouages?

D'autre part, l'étude de la structure, si elle n'était pas suivic de celle de la fonction, n'arriverait pas à satisfaire l'esprit, car elle ne constituerait forcément qu'une notion incomplète ; ce serait quelque chose d'analogue à l'énoncé d'un principe dont on ne tirerait pas les conclusions. On n'apprendrait ainsi à connaître qu'une partie de l'être et non pas l'être lui-même, tel que la nature nous le présente.

Aussi le programme officiel de l'étude de la Botanique pour la Classe de Philosophie veut-il que, tout en insistant sur les questions de Physiologie végétale, l'élève ne néglige pas celles d'Anatomie, dont l'utilité n'est pas douteuse pour qu'il puisse se faire des premières une idée juste et suffisante.

Nous suivrons donc de près les données de ce programme; et toutes les notions anatomiques qui ont pris -place au commencement de ce livre doivent être regardées comme une introduction indispensable aux questions relatives à la physiologie.

- Les Végétaux sont des êtres vivants, et leur étude rentre dans la Biologie, vaste science, qui comprend en même temps celle des Animaux, que tant de caractères, on doit le constater, rapprochent des premiers.

En effet, en sa qualité d'être vivant, la Plante naît, respire, se nourrit, se reproduit et finit par mourir : autant d'opérations que nous rencontrons également chez l'Animal, et qui séparent nettement, comrne par une barrière infranchissable, les deux règnes organisés du monde des êtres inorganiques.

Bien plus, quoique la distinction entre la plupart des animaux et des végétaux paraisse, au premier abord, des plus faciles, un examen un peu approfondi des uns et des 
autres montre relativement à un groupe important de fonctions, celles qu'on appelle végétatives ou de nutrition, la similitude la plus frappante, au point que les différences que l'on peut noter dans ce travail de comparaison ne portent que sur des particularités de second ordre.

On ne sera pas étonné outre mesure de ce résultat, quand on aura appris que, chez les Végétaux comme chez les Animaux, l'organisme est formé d'éléments arratomiques ou cellules, contenant une substance spéciale, le protoplasma, agent indispensable des manifestations vitales, et doué d'un grand nombre de propriétés communes aux uns et aux autres; ce qui n'empêche pas toutefois de reconnaître que le protoplasma végétal, d'une part, le protoplasma animal, de l'autre, doivent posséder, en outre, des propriétés spéciales, mais que l'imperfection de nos sens et de nos instruments de recherche ne nous permet pas de mettre en évidence.

D’ailleurs, cette question ayant été déjà suffisamment traitée dans le Cours de Zoologie, nous n'y insisterons pas davantage ici (1).

C'est en vain que l'on chercherait dans la vie sédentaire des plantes un critérium absolu qui séparerait d'une façon rigoureuse le règne végétal du règne animal, car tandis que certaines plantes, d'ordre inférieur, il est vrai, se meuvent et se déplacent avec une rapidité variable suivant les espèces (Voy. Chap. xiv), on connaît nombre d'animaux également sédentaires, fixés au sol comme les végétaux, ce qui les avait fait ranger pendant longtemps parmi

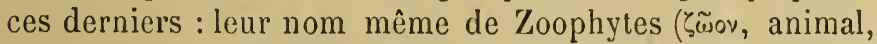
yutòv, plante) rappelle l'analogie qu'ils offrent, à ce point de vue, avec les végétaux.

L'absence d'un tube digestif ou d'un appareil circulatoire véritable, etc., ne fournit pas davantage le signe caractéristique que nous cherchons, car les fonctions auxquelles ces organes se rapportent s'accomplissent chez les Plantes

(1) Cours de Zoologie, Anatomie et Physiologie animales, par le Dr P. Maisonneuve. Voy. Introduction, p. xvr, et Chap. vu, p. 193 à 202 . 
aussi bien que chez les Animaux; et du reste si ces appareils eux-mêmes manquent chez les Plantes, il s'en faut bien qu'ils se rencontrent chez toutes les espèces animales.

Pour nous résumer, répétons que les fonctions végétatives ou de nutrition sont communes aux deux règnes organiques.

Si donc, nous voulons tenter d'établir une marque de séparation entre ces deux groupes, nous devons la chercher dans une sphère plus élevée, dans ces hautes fonctions qui sont l'apanage des seuls Animaux, et qu'on appelle, en raison de cette circonstance, les propriétés animales; à savoir, la sensibilité et le mouvement volontaire.

Et si, dans les Plantes, les manifestations extérieures d'une sorte d'irritabilité dont l'existence n'est pas contestable chez elles, semblent devoir faire illusion, du moins à un examen superficiel, nous espérons montrer, qu'en réalité, la Plante ne sent pas, de même qu'elle ne se meut pas volontairement. Aussi, malgré les rapports certains qui unissent les deux grands règnes vivants entre eux, devonsnous reconnaître qu'ils présentent, par contre, une différence fondamentale, bien qu'il ne soit pas toujours facile d'en faire sans hésitation l'application aux cas particulier's.

G'est pourquoi, avec Linné, qui avait cependant si bien saisi les nombreuses relations qui enchaînent les uns aux autres les divers êtres créés, ce qui l'avait amené à poser l'axiome célèbre : "Natura non facit saltus ", on doit admettre que les trois règnes de la nature sont séparés l'un de l'autre par l'apparition d'un caraclère d'ordre d'autant plus élevé que l'on va du règne inférieur au règne le plus parfait, et dire avec le grand naturaliste suédois : Mineralia crescunt; Vegetalia crescunt et vivunt; Animalia crescunt, vivunt et sentiunt. 


\section{TRAITE DE BOTANIQUE}

A NATOMIE

ET

PHYSIOLOGIE VÉGÉTALES 



\section{A NATOMIE}

ET

\section{PHYSIOLOGIE VÉGETALES}

\section{H A P ITRE I}

\section{Éléments anatomiques des Végétaux.}

La Cellule est la base de tout organisme végétal. Constitution de la Cellule: Protoplasma, Vacuoles et Courants protoplasmiques; Suc cellulaire; Noyau et Nucléole. Membrane d'enveloppe; ses modifications de structure : cellules ponctućes, rayées, aréolées, etc. - Fibres. - Vaisseaux. - Laticifères. - Les Tissus des végétaux.

LA CEllule est la base de tOut ORganisme végétal - Si l'on étudie à l'aide du microscope une plante quelconque, qu'elle appartienneàux plus humbles parses dimensions, aux plus simples par sa structure, un Champignon, ou une Algue, par exemple, ou qu'elle soit prise parmi les familles les plus parfaites du règne végétal, comme le Chêne, l'Orme, etc.; que l'on borne ses recherches à l'examen de la tige, de la racine, ou bien qu'on les poursuive dans les feuilles et les organes de la fleur, on est amené à reconnaître que l'organisation de tout végétal se résout, en somme, en une infinité de petits corps distincts, que l'on appelle les éléments anatomiques. 
Tandis que parmi les plantes, les unes en comptent un nombre pour ainsi dire infini, il en est d'autres qui ne sont formées que d'un petit nombre, certaines même d'un scul de ces éléments (fig. 1).

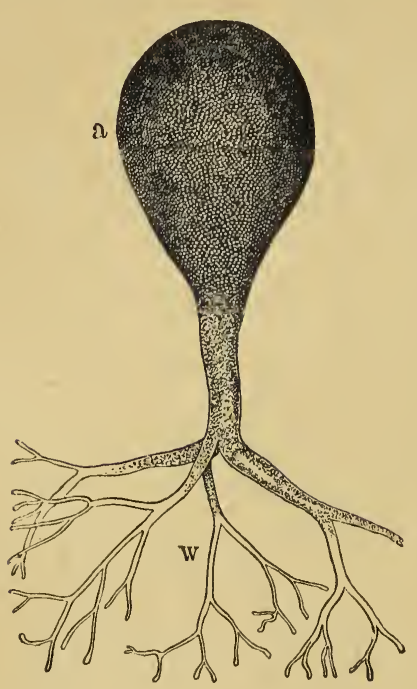

Fig. 1. - Une Algue unicellulaire (Botrydium granulatum) grossic 15 fois; $\boldsymbol{a}$, partie supéricure; $w$, racines.

Cette réduction de l'organisation végétale en éléments anatomiques établit une grande analogie entre les plantes et les animaux au point de vue de la struclure, les premières comme les seconds n'étant en définitive qu'un échafaudage de ces petits corps (1). On verra, d'ailleurs, chemin faisant, qu'il y a bien d'autres rapports saisissants entre les êtres des deux règnes organiques.

Le plus simple des éléments anatomiques et celui dont tous les autres dérivent est la cellule; c'est donc par elle qu'il convient de commencer.

L'étude de la cellule va être divisée en trois parties:

$1^{\circ}$ la constitution de la cellule elle-même et des éléments anatomiques qui en dérivent;

2. l'agencement des cellules ou de leurs dérivés pour constituer les tissus;

$3^{\circ}$ les produits cellulaires.

Constitution de la cellule. - Les cellules peuvent offrir undegré de complication variable. Dans toute cellule complète on peut considérer deux parties, à savoir: un contenant,

(1) Voycz Cours de Zoologic. Anatomie et Physiologie animales, chap. ViII, p. 203, par le Dr P. Maisonneuve, Palnić, 1883. 
l'enveloppe de la cellule ou paroi cellulaire, et un contenu, le protoplasma avec les parties qui en dépendent.

Protoplasma. - Le proloplasmá (1) a été défini par un illustre naturaliste anglais, Huxley, "la base phiysique de la Importance vie »; c'est qu'en effet, cette substance est la partie fondamentale de tout organisme, celle sans laquelle la vie ne peut pas se produire ou se maintenir. On le rencontre chez les végétaux aussi bien que chez les animaux, et avec des caractères objectifs si manifestement identiques dans les deux groupes, que dans l'état actuel de la science on ne peut ćtablir de différence essenticlle de structure et de fonctions entre l'un et l'autre. C'est même là une preuve, pour le dire en passant, qu'il y a dans cette substance douée de vie, une force que nos moyens ne nous permettent pas de saisir, mais qui n'en existe pas moins, car si l'identité du protoplasma végétal et du protoplasma animal était absolue, il n'y aurait pas de raison pour que, considéré dans le germe, le premier donnât toujours naissance à une plante, et l'autre à un animal, plutôt que d'évoluer toús deux dans le même sens.

Vu au microscope, lc protoplasma a l'aspect d'une gouttelette de substance mucilagineuse (fig. 2), incolore ou opaline, quelquefois teintée.

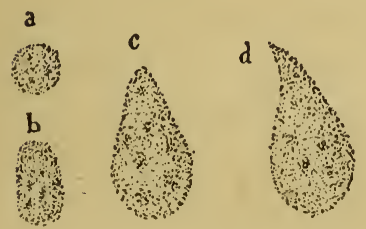

Fig. 2. - Protoplasma de cellules nues $(a, b, c, d)$. phore et quelques autres corps moins fréquents ou en moindre quantité. se compose d'Oxygène, d'Hydrogène, d'Azole et de Carbone; il fait done partie des substances albuminoïdes, appelées encore substances quaternaires, azotées, protéiques. Comme éléments accessoires on y trouve du Soufre, du Phos-
Caractères pliysiques et chimiques.

La chimie nous apprend qu'il

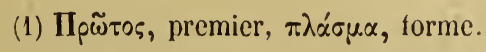


Sous l'influence de la chaleur, de l'alcool, des acides minéraux dilués, il se coagule. En présence des acides il offre des réactions caractéristiques: il perd promptement ses propriétés vitales, mais se colore de diverses façons, en jaune par l'acide azotique, couleur qui s'accentue si on le traite ensuite par l'ammoniaque ou la potasse, en rougeâtre par l'acide sulfurique, en rose violet par l'acide chlorhydrique. Les alcalis, tels que la potasse ou l'ammoniaque, le détruisent promptement, réaction caractéristique des substances albuminoïdes, telles que la fibrine, la caséine, l'albumine.

Propriétés organiques.

Le protoplasma est essentiellement la substance vivante de l'organisme végétal; il est le siège des différents actes vitaux en vertu desquels la plante se nourrit et se développe. Toute cellule qui renferme du protoplasma vit; toute cellule qui en est dépourvue ne vit plus, elle est morte; ce n'est qu'un squelette désormais non susceptible d'accroissement et incapable de quelque manifestation vitale que ce soit.

Le protoplasma respire, c'est-à-dire qu'il puise dans le monde extérieur certains gaz (oxygène), et en dégage certains autres (acide carbonique). Il se nourrit en absorbant des substances gazeuses ou liquides, qu'il puise dans l'air atmosphérique ou dans le sol. C'est de lui que dépend l'accroissement de la plante, car sous l'influence de la nourriture qu'il absorbe, il augmente de volume et donne naissance à de nouvelles petites masses protoplasmiques.

Un caractère particulièrement frappant du protoplasma végétal et qui le rapproche manifestement de celui de l'Animal, c'est la propriété qu'il a de se contracter, bien plus, de se mouvoir et de se déplacer. Ses mouvements ont reçu le nom d'amiboïdes, parce qu'ils rappellent, d'une manière frappante, ceux des animaux inférieurs appelés Amibes. Cette propriété se constate facilement dans les cellules de grande dimension, des Chara, par exemple, et surtout dans certains champignons inférieurs, les Myxomycètes, qui voyagent d'un endroit à l'autre, lentement, au moyen d'un glissement continu, enfin dans les anthérozoïdes ou corpuscules reproducteurs d'un grand nombre de plantes inférieures, qui 
nagent avec unc grande vivacité dans les eaux où ils tombent (1).

La chaleur, la lumière, l'état électrique de l'air ont une grande influence sur les manifestations vitales du protoplasma. La façon dont il réagit sous l'action des divers agents extérieurs est telle qu'il paraît doué d'une sensibilité particulière dont les manifestations sont, jusqu'à un certain point, comparables à celle des animaux. C'est ainsi que lorsqu'il est dans des conditions à pouvoir se déplacer, il se dirige vers la lumière ou la fuit au contraire lorsqu'elle est trop vive; c'est à lui surtout que sont dus les changements de situation éprouvés par les feuilles pendant le jour et pendant la nuit. On verra au Chapitre xir, qui traite de cette question, qu'il n'y a pas là de mouvement volontaire, mais simplement une réaction causée par un agent physique ou chimique; que ce mouvement est toujours approprié à un but; qu'il n'est pas voulu par la plante, laquelle n'y peul rien changer, bien qu'il offre parfois, d'une façon singulière, les apparences de la spontanéité, et qu'en réalité, les animaux les plus inférieurs qui touchent, comme on sait, de si près au règne végétal, n'en présentent pas de beaucoup plus manifestement volontaire.

Vacuoles et Courants protoplasmiques; Suc cellulaire.
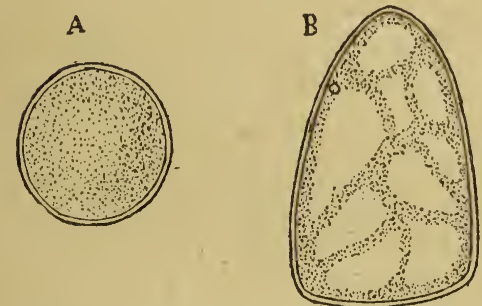

Fig. 3. - A, Cellule dont le protoplasma remplit toute la cavité. $B$, Cellule dont le protoplasma est creusé de vacuoles.
- Quand la cellule est jeune, c'est-à-dire quand elle vient de prendre naissance, le protoplasma la remplit exactement (fig. $3, A$ ). Il n'en est pas de même un peu plus tard; et si l'on examine une cellule un peu plus âgée, on voit que le protoplasma, au lieu de former une gouttelette uniforme, homo-

(1) Voy. Chap. xII. Mouvement et Sensibilitê des Végétaux. 
gène, se creuse de vacuoles et prend la forme d'un petit sac, en même temps que des prolongements ou rubans protoplasmiques partent de différentes régions de cette enveloppe pour se réunir les uns aux autres $(\boldsymbol{B})$.

Dans la zone périphérique du pro-

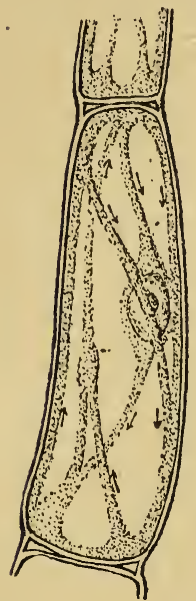

Fig. 4. - Cellule avec protoplasma creusé de vacuoles et disposé en rubans le long desquels se produisent des courants dont la direction est indiquéc par les flèches. toplasma, aussi bien que dans les rubans protoplasmiques se produisent des courants (fig. 4), que l'on peut suivre grâce ầ la présence de très fins corpuscules qui s'y trouvent en suspension et qui sont entraînés par ces courants; il y a donc une véritable circulation intra-cellulaire. D'autres fois on constate un mouvement d'ensemble de toute la masse protoplasmique qui se déplace dans sa totalité par un mouvement de glissement contre la paroi cellulaire, lequel a reçu le nom de rotation du protoplasma (fig. 5).

Les espaces creusés dans le protoplasma ne sont pas vides, mais remplis d'un liquide aqueux, transparent, qu'on appelle le suc cellulaire. Il se compose surtout d'eau renfermant en dissolution certains principes, notamment des sels, naguère contenus dans le protoplasma et qui en ont été excrétés. Le suc cellulaire est le milieu dans lequel s'accumulent les aliments qui ont été absorbés en excès et qui ne trouvent pas un emploi immédiat, soit qu'ils s'y transforment en corps gras ou en fécule, soit qu'ils y restent à l'état de solution. C'est dans ce milieu que le protoplasma puise, suivant ses besoins, les aliments nécessaires au maintien de sa constitution normale et à son accroissement.

Noyau ét Nucléole. - Le plus souvent, outre les parties quı viennent d'être passées en revue, la cellule renferme 
un petit corps, en général arrondi ou ovalaire, enfoui dans le protoplasma. Ge petit corps, qui

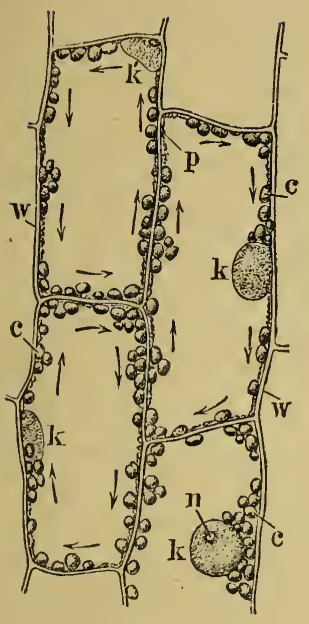

Fig. 5. - Cellules de feuille de Vallisneria spiralis montrant la rotation du protoplasma; $w$, paroi cellulaire; $p$, protoplasma; $c$, grains de chlorophylle; $k$, noyau cellulaire; $n$, nucléole. Les flèches indiquent la direction suivie par le protoplasma. se distingue nettement au microscope par sa réfringence ordinairement bien plus grande que le milieu dans lequel il est plongé, porte le nom de nucléus ou de noyau. Toujours enveloppé de protoplasma, il est situé dans la zone périphérique de celui-ci, ou bien se trouve en quelque sorte soutenu au milieu de la cellule par des rubans protoplasmiques qui se réunissent sur lui (fig. 4, 5 et 6 ).

Le noyau paraît être de la nature même du protoplasma, mais à un état de plus grande condensation. Comme il a la propriété d'absorber plus énergiquement les matières colorantes que le protoplasma, on a par là un moyen trèsfacile de le mettre en évidence dans les préparations microscopiques, pour s'en faire une idée plus complète. D'autre part, l'acide acétique lui donne une netteté remarquable, en même temps que le reste du protoplasma est dissous par ce réactif. Le noyau a donc des caractères communs avec le protoplasma et en diffère nettement par certains autres.

Ses fonctions sont encore enveloppées d'obscurité; il paraît, en tout cas, jouer un rôle important dans la vie de la. cellule et surtout dans sa multiplication, d'où le nom de

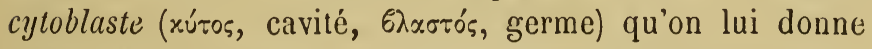
souvent. Ajoutons qu'au lieu d'un seul noyau, on en rencontre parfois deux et mêıne plus dans une même cellule.

Enfin le noyau offre le plus souvent dans son intérieur un, Nuclíoles. deux ou plusicurs corpuscules beaucoup plus petits que lui, 
qui apparaissent sous forme de petits points brillants, qu'on appelle des nucléoles, mais sur le rôle desquels on n'est nullement fixé (fig. 6).

Constitution physique et chimique.
Membrane d'enveloppe ou paroi cellulaire. - Il est des cas où le protoplasma reste, du moins pendant un certain temps, complètement nu, c'est-à-dire qu'à lui seul il constitue toute la cellule et n'est séparé par aucune barrière du monde extérieur, avec lequel il communique directement (fig. 2). Mais le plus souvent, au bout de peu de temps il s'entoure d'une enveloppe qu'il sécrète lui-même et qu'on appelle paroi cellulaire ou membrane d'enveloppe (fig. $3,4,5$ et 6 ).

Désormais protégé par cette mem-
Fig. 6. - Cellules montrant un noyau avec un ou deux nucléoles.

brane, c'est à travers les pores qu'elle présente que le protoplasma communiquera avec l'extéricur pour opérer les échanges gazeux de la respiration et accomplir les phénomènes de nutrition. Celte enveloppe est souvent d'une extrême minceur et très molle, surtout quand elle est de formation récente; mais, par la suile, elle s'épaissil et acquiert une grande dureté. Il en résulte qu'elle devient incapable de se laisser pénétrer par les gaz et les liquides; aussi arrive-t-il un moment où le protoplasma en a disparu; il ne- reste plus, en quelque sorte, que la coque de la cellule, une demeure vide, dont l'habitant est sorti.

Quoiqu'il en soit, la membrane cellulaire est forméc de cellulose, substance ternaire, et a la même composition chimique que la fécule $\left(\mathrm{G}^{12} \mathrm{H}^{10} \mathrm{O}^{10}\right)$. Elle se dissout dans l'acide sulfurique et se gonfle sans se dissoudre dans une solution de potasse caustique; traitéc par la teinture d'iode et l'acide sulfurique, elle offre une coloration bleue caractéristique.

L'apparence de la paroi cellulaire, et par suite, de la cellule, est très variable.Quand elle est de formation récente, 
elle est arrondie, ovoïde ou oblongue (fig. 7 ); mais bientôt elle devient cylindrique ou polyédrique par suite de la compression exercée sur elle par les cellules voisines;

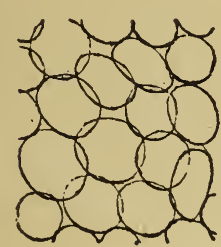

Fig. 7.

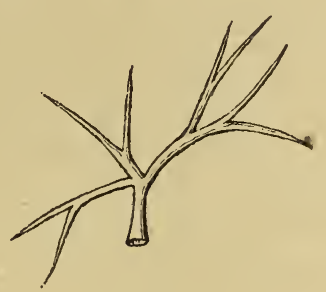

Fig. 8.

Fig. 7. - Groupe de cellules à parois minces et à contours arrondis.

Fig. 8. - Cellule rameuse (un poil de Crucifère).

d'autres fois elle offre des prolongements qui lui donnent un aspect rayonné, rameux, comme c'est le cas des cellules qui constituent les poils d'un certain nombre de plantes (fig. 8).

Modifications présentées par la parol cellulare. Comme on l'a dit un peu plus haut, la paroi cellulaire ne reste pas longtemps mince; le plus souvent elle s'épaissit (fig. 9) par suite du dépôt, dans l'épaisseur de ses parois,

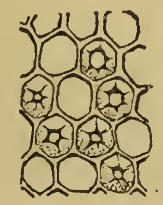

Fig. 9.

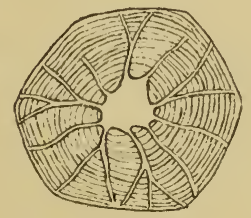

Fig. 10.

Fig. 9. - Groupe de cellules à parois épaisses et à contours polyédriques.

Fig. 10. - Cellule á paroi épaisse creusée de fins canaux.

de certaines matières, le plus souvent du ligneux, substance très analogue à la cellulose et de même composition chimique, qui constitue la partie essentielle du bois des végé- 
taux. Mais parfois aussi des substances minérales peuvent s'y déposer, telles que de la silice, du calcairc, et souvent des matières colorantes.

La paroi cellulairc épaissie paraît à un fort grossissement composéc d'un certain nombre de couches superposées, qui semblent, au premier abord, dues à des dépôts successifs venant se recouvrir les uns les autres (fig. 10). Il n'en est rien, en réalité : l'épaississement de la paroi cellulaire se fait, non par superposition de parties, mais par intussusception. On entend par là que les substances absorbées qui viennent accroître la paroi de la cellule, au lieu de se déposer à la surface soit extérieure, soit intérieure, pénètrent molécule à molécule dans l'épaisseur même du tissu. L'aspect zonaire est dû à un phénomène secondairc qui se produit après que la membrane a acquis une certaine épaisseur; il est dû à une alternance de couches plus denses et de couches moins denses.

Le plus souvent, cet épaississement de la paroi cellulaire ne se fait pas d'une façon uniforme, mais seulement dans c ertaines régions à l'exclusion de certaines autres qui, dès lors, vues par transparence, paraissent plus claires, tandis
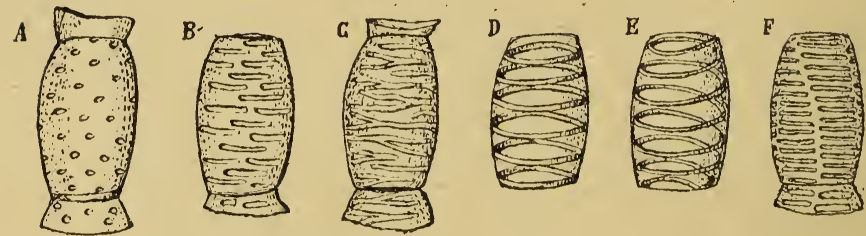

Fig. 11. - Cellules présentant des épaississements divers. $A$, cellule ponctuéc; $B$, cellule rayéc; $C$, cellule réticulée $; D$, cellule anneléc; $E$, cellule spiralée; $\boldsymbol{F}$, cellule scalariforme.

que les premières offrent une teinte plus sombre; de là une très grande variété dans l'aspect de la membrane (fig. 11). Ainsi, par exemple, lorsque les parties non épaissies sont de simples points $(A)$, li paroi semble comme criblée de petite perforations dues à la minceur de ces parties, et la cellule est dite ponctuée; si les parties non envahies par 
l'épaississement ont la forme de lignes $(\boldsymbol{B})$, la cellule est dite rayée. Il peut se faire, par contre, que l'épaississement se produise seulement suivant des lignes affectant la forme de réseaux, d'anneaux ou de spirales $(C, D, E)$, ce qui donne les cellules réticulées, annelées, spiralées; enfin si les lignes d'épaississement ont la forme de lignes régulièrement disposées comme les rayons d'une échelle $(\boldsymbol{F})$, la cellule est appelée scalariforme.

Une des dispositions les plus remarquables est celle dans laquelle les cellules ponctuées présentent chacune de Cellules aréolées leurs ponctuations entourée d'un zone claire (fig. 12), d'où le nom d'aréolées, qu'on leur donne. Cette disposition, qui est fréquente dans le bois des Conifères ou arbres verts, tient à ce que la paroi qui sépare deux cellules voisines (fig. $13, A, b)$ restant très mince sur une faible étendue,
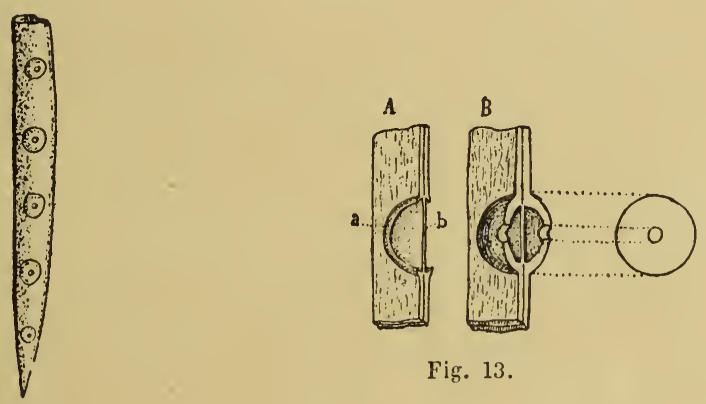

Fig. 13.

Fig. 12 .

Fig. 12. - Ponctuations aréolées sur une fibre ligneuse.

Fig. 13. $-A, B$, Ces dessins sont destinés à montrer la formation des aréoles, dues à la production, autour d'une ponctuation ordinaire $(b)$, d'un bourrelet circulaire (a) qui s'accroît de plus en plus de façon à limiter un étroit orifice central.

s'épaissit tout autour de cet espace: l'espèce de bourrelet circulaire $(a)$ qui en résulte s'avance au-dessus de la membrane primitive $(b)$ qu'il surplombe, en resserrant de plus en plus son orifice. Il cn résulte l'apparence de deux cercles concentriques, l'un plus griand, qui répond à l’épaississement circulaire, l'autre plus petit et concentrique au premier, répondant à la portion non épaissie de la membrane primitive vue à travers l'orifice du bourrelet. 
Elles dérivent des cellules.

Structure des tibres.

Fibres. - Lorsque par les progrès de leur développement, les cellules, comprimées, serrées les unes contre les autres, s'allongent en une sorte de tube terminé en pointe à chaque extrémité, de façon à affecter la forme d'un fuseau, on leur donne le nom de fibres (fig. 14, $A, B$ ). Les fibres ne sont donc pas des éléments anatomiques absolument différents des cellules; ils n'en sont au contraire que des modifications.

Les fibres serrées les unes à côté des autres avec leurs extrémités aiguës intercalées entre celles des fibres placées

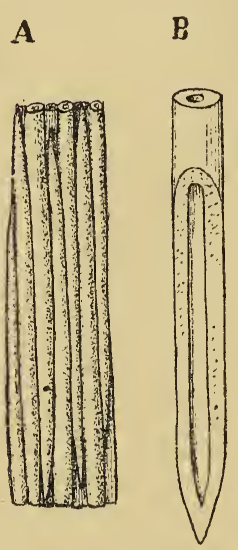

Fig. 14. - $A$, fibres serrées et accolées entre elles; $B$, une cellule isolée et coupée dans une partie de sa longueur pour en montrer la structure.

au-dessus et au-dessous, adhèrent fortement entre elles, sont souvent ramifiées et constituent des faisceaux fort résistants. En outre, les parois des fibres sont très épaissies, de sorte que le canal central dont chaque fibre est creusée est fort étroit. La plus grande partie du bois des végétaux est formée par ces éléments, au milieu desquels sont intercalés les vaisseaux dont il va être bientôt question. Ce sont les fibres aussi qui constituent ces filaments si résistants que fournissent les parties corticales d'un grand nombre de plantes et qui servent à la confection des étoffes (Lin, Chanvre), ou à la fabrication des cordages (Chanvre, Aloès, etc.). Enfin ce sont ces mêmes éléments qui composent, pour la plus grande partie, les nervures des feuilles.

Puisque les fibres dérivent des cellules, dont elles ne sont qu'une modification, on ne sera pas surpris d'apprendre que leurs parois offrent toutes les particularités que les parois des cellules nous ont déjà montrées, et qu'il y en a de ponctuées, de rayées, d'aréolées, elc. C'est même chez elles que cette dernière disposition se rencontre le plus communément (fig. 12). 
Vaissenux. - Comme les fibres, les vaisseaux dérivent Ils dérivent des des cellules. Ils résultent en effet de la réunion bout à bout d'un certain nombre de ces dernières, entre lesquelles il s'établit une large communication par suite de la disparilion des parois interposées entre leur's cavités.

Les vaisseaux constituent des tubes très fins, fort longs et qui, contrairement aux fibres, ne sont, à l'exception de ceux qu'on appelle laticifères, jamais ramifiés.

Comme les cellules qui le composent, un vaisseau peut être ponctué, rayé, spiralé ou réticulé (fig. 15, $a, b, c, d$ ).

Structure des vaisseaux.

Trachées.

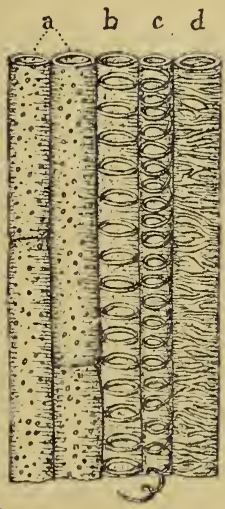

Fig. 15.

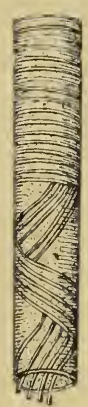

Fig. 16 .

Fic. 15. - Vaisscaux ponctués, annelé, spiralé, ríticulé $(a, b, c, d$.)

Fıg. 16. - Un vaisseau spiralé (feuille de Bananier.)

Ceux qui présentent la disposition spiralée $(c)$ méritent d'arrêter quelques instants l'attention. L'épaississement, dans ce cas, s'est produit sous forme d'un petit cordon spiral qui fait saillie dans l'intérieur du vaisseau. Sa résistance est assez grande, et son adhérence à la paroi assez faible pour quon puisse par la traction le dérouler au dehors comme un petit ressol't, ou bien c'est la paroi même du vaisseau qui se déchire suivant la spirale en question. C'est ce qu'il est on ne peut plus facile de reconnaître en déchirantavec quelque précaution un pétale de rose: on 
voit alors que les deux fragments sont réunis par des fils élastiques d'une finesse extrême, qui ne sont autre chose qne les petits épaississements spiraux qui se déroulent. Dans la feuille du Bananier ils sont encore bien plus faciles à voir, car au lieu d'un seul filament par vaisseau, il y en a plusieurs accolés les uns aux autres (fig. 16) et se déroulant sous forme d'un ruban. Les tours de spire sont plus ou moins espacés; parfois ils sont si serrés qu'ils recouvrent entièrement la paroi du vaisseau.

On a trouvé une certaine analogie entre les vaisseaux spiralés des plantes et les tubes respiratoires ou trachées des Insectes, qui sont, en effet, garnis d'un fil très fin enroulé de la sorte; aussi a-t-on appelé trachées les vaisseaux des plantes qui offrent celle disposition; quant au filament luimême, il porte le nom de spiricule. Malgré leur finesse, les spiricules paraissent, au moins dans certains cas, être creusés d'un canal dans toute leur longueur. Les trachées ne sont pas indifféremment répandues dans toutes les parties des végétaux : daus les Dicotylédones elles s'observent seulement dans cette partie de la tige qui entoure la moelle et qu'on appelle l'étui médullaire, tout le reste du bois et de l'écorce en étant dépourvu. Ghez Ies Monocotylédones on les trouve dans tous les faisceaux fibro-vasculaires disséminés dans la tige. Les nervures des feuilles et diverses parties des fleurs en contiennent toujours; on en rencontre enfin dans les racines.

Rôle des vaisseaux.

La paroi des vaisseaux, contrairement à celle des fibres reste, en général, mince; lorsque ces organes sont encore jeunes, ils sont remplis de suc, ou pour mieux dire de sève; c'est par leur intermédiaire en effet que ce liquide monte; un peu plus tard, la sève les abandonne el lcur cavité ne renferme plus que des gaz. (Voyez Chap. V, Circulation.)

Vaisseaux laticifères. - Tandis que les vaisseaux qui viennent d'être étudiés ne communiquent en aucune façon les uns avec les autres et forment autant de tubes continus à peu près rectilignes, il en est d'une certaine catégorie qui 
s'abouchent par leurs parois latérales, se rejoignent par des branches transversales, de façon à former un système ramifié; ce sont les vaisseaux laticifères (fig. 17). Ils dérivent

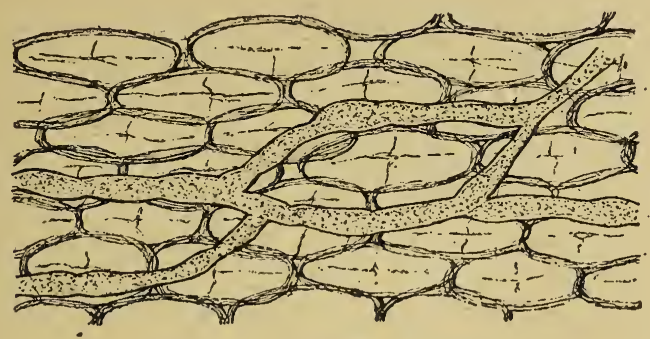

Fig. 17. - Vaisseaux laticiferes.

de cellules glandulaires, c'est-ì-dire de cellules spéciales destinées à la sécrétion de certaines substances. Placées en file, leurs parois de séparation se sont résorbées en totalité ou en partie de façon à établir entre elles une facile communication. On trouve de semblables vaisseaux, par exemple, dans les feuilles et les écailles de l'Ail commun, dans les feuilles de l'Aloès, où ils paraissent chargés de la secrétion du principe amer qui porte le nom de cette plante, dans l'Érable, où ils fournissent un liquide sucré, etc. D'autres fois les files de cellules sécrétrices sont reliées par d'autres, disposées dans le sens transversal, de façon à constituer un réseau à mailles plus larges ou plus serrées suivant les espèces de plantes. Cies réseaux sont sourent très sinueux et très compliqués, pourvus d'un grand nombre de ramifications anastomosées entre elles ou terminées en cul-de-sac: telle est la disposition que l'on rencontre dans les Pavots, les Laitues.

Les parois de ces vaisseaux sont ordinairement fort minces, sans poncluations ou épaississements quelconques; leur calibre, au lieu d'être uniforme, présente des alternances de dilatation et d'étranglement qui ont mérité à ces canaux l'épithète de moniliformes.

On les rencontre d'ordinaire dans l'écorce des Dicolylédones, surtout vers sa partie interne, tandis que chez les 
Monocotylédones ils se voient parmi les faisceaux vasculaires disséminés dans la tige. Les nervures des feuilles en prẻsentent souvent. Dérivés des cellules comme les précédents, ils renferment un suc propre ou latex, liquide bien différent de la sève. Il sera étudié à la fin du chapitre II.

\section{Les Tissus.}

Différentes SORTES DE tissus. - Il ne suffit pas, pour connaître la structure des plantes, d'avoir appris quels en sont les éléments conslituants. Il n'est pas moins nécessaire de savoir comment ces éléments sont groupés entre eux et quels sont leurs rapports réciproques.

Serrés les uns contre les autres, confondant souvent leurs parois, communiquant ou non entre eux par leurs cavités, ces éléments constituent les tissus. Ceux-ci sont au nombre de trois principaux:

Lorsque des cellules composent à elles seules le tissu, c'est le tissucellulaire ou utriculaire, a ppelé encore parenchyme (1);

Lorsqu'il est formé par les éléments que nous avons appelés des fibres, c'est le tissu fibreux ou prosenchyme (2);

Enfin, s'il est constitué par des vaisseaux, il prend le nom de tissu vasculaire.

Nous avons vu comment les tissus fibreux et vasculaires se forment aux dépens du tissu cellulaire. Il nous reste maintenant à étudier comment se produit lui-même ce dernier, point de départ de tous les autres.

Tissu cellulaire; son mode de formation. - Le tissu cellulaire est dû à l'apparition de cellules nouvelles résultant de la multiplication des cellules préexistantes. Voyons donc par quels procédés se multiplient, s'engendrent, si l'on veut, les cellules qui le constituent.

(1) Ilapś $\gamma \chi u \mu \alpha$, substance des organes.

(2) IIpós, impliquant lidée de force, $\varepsilon^{\prime} \gamma / u \mu \alpha$, substance. 
Si l'on examine au microscope ces Algues vertes filamenteuses (Conferves) qui abondent dans les eaux stagnantes, et dont chacune se compose uniquement d'une rangée de cellules mises bout à bout,

A

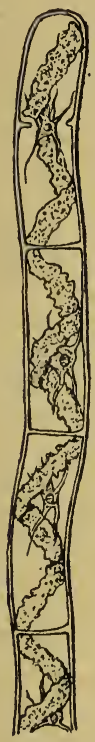

Fig. 18. - Multiplication cellulaire par division transversale; $\boldsymbol{A}$, une portion d'Algue filamenteuse (Conferve); $B$, cellule terminale en voie de division; $C$, la formation des deux cellules est complite. on peut suivre toutes les phases de la formation cellulaire (fig. 18). Et d'abord il n'y a qu'une seule cellule qui ait la faculté d'en produire d'autres; c'est toujour's la dernière formée, celle qui occupe le sommet du filament (A). On remarque que le noyau et le protoplasma qui l'entoure s’allongent un peu, s'étranglent en quelque sorte (B), puis se séparent en deux portions, en même temps qu'une cloison membraneuse se forme pour séparer les deux nouveaux noyaux et le ur protoplasma, de façon à constituer une cellule nouvelle $(\mathrm{C})$.

Il peut se faire que la segmentation de la cellule commence par la membrane d'enveloppe ; le noyau et le protoplasma ne subissant qu'après elle la même opération.

La formation des cellules ne procède pas toujours comme il vient d'être indiqué. Parfois, le noyau et le protoplasma se divisent en deux ou plusieurs parties, sans qu'il y ait en même temps ou auparavant production d'une cloison de cellulose, celle-ci ne se formant que plus tard de façon à entourer chaque petite masse d'une enveloppe complète; c'est ce qu'on appelle la formation cellulaire libre (fig. 19). 
Une fois formée, chaque nouvelle cellule grandit, puis ne tarde pas à se diviser à son tour, pourvu que la nutrition soit

A

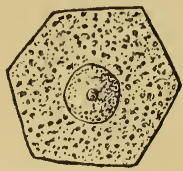

B

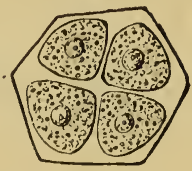

G

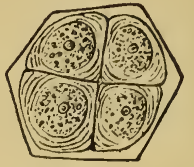

Fig. 19. - Multiplication des cellules par formation libre; $A$, cellule avec noyau; $B$, cellule dont le protoplasma se divise en quatre; $C$, formation des cloisons qui séparent les nouvelles cellules.

suffisamment active; c'est ainsi que se fait l'accroissement de la plante.

DIFFÉRENTES SORTES de TISSU CELlulaire. - Le tissu cellulaire ou parenchyme offre dans son aspect un certain nombré de variélés qui liennent à la

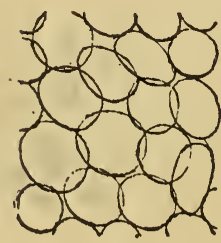

Fig. 20. - Tissu cellulaire ou parencliyme arrondi. forme et au mode de groupement des éléments constituants. Quand elles sont jeunes, les cellules offrent la forme arrondic ou ovalaire et constituent le tissu cellulaire arrondi (fig. 20); les plantes grasses, les fruits pulpeux en offrent de bons exemples. Dans ce cas, des espaces vides plus ou moins considérables se trouvent interposés entre les points de contact des cellules; ce sont les méats intercellulaires.

Le plus souvent, par suite de leur accroisssement et de leur multiplication, les cellules se compriment réciproquement et forment un parenchyme polyédrique, hexagonal (fig. 21); la moelle des plantes en offre des exemples caractéristiques. D'autres fois, les cellules sont disposées comme les pierres de taille d'une muraille et constituent le parenchyme muriforme (fig. 22). Les cellules peuvent présenter des prolongements plus ou moins irréguliers, d'où le 
nom de parenchyme rameux (fig. 23); et si ces prolongements sont disposés avec une certaine régularité, on a le

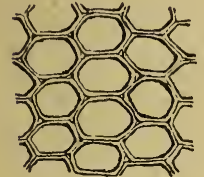

Fig. 21

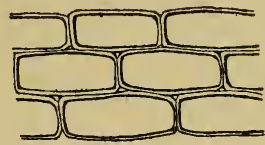

Fig. 22.

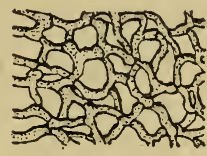

Fig. 23.

Fıg. 21. - Parenchyme polyédrique.

Fig. 22. - Parenchyme muriforme.

Fig. 23. - Parenchyme rameux.

parenchyme étoilé (fig. 24), qui s'observe avec une trèsgrande netteté dans la tige du Jonc des marais; les larges espaces vides qui résultent de cette disposition permettent aux gaz de circuler entre les cellules.

Matière intercellulaire. - Les cellules sont réunies les unes aux autres, sont accolées,

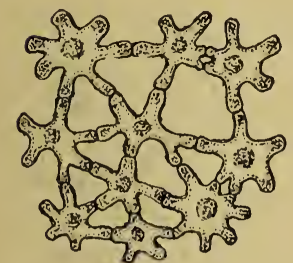

Fig. 21. - Parenchyme à cellules étoilées. soudées plus ou moins intimement, de façon à former un tissu. Ce résultat est obtenu par l'interposition, entre les cellules, d'une substance que l'on a considérée pendant longtemps comme en étant distincle, et qui se serait épanchée entre elles pour les agglutiner à la manière d'un ciment, d'où le nom de matière intercellulaire qu'on lui a donné. On peut conserver ce nom, mais on devra se rappcler que cette substance n'est autre chose que la couche extérieure plus molle des parois cellulaires. Son abondance varic beaucoup avec les tissus; d'ordinaire très peu épaisse, elle se trouve au contraire en grande quantité dans les tissus mous, mucilagineux, comme on en rencontre dans cerlaines Algues.

Répartition des différents tissus. - Les différents 
tissus qui viennent d'être étudiés sont loin de se trouver également répartis dans tous les végétaux et dans les différents organes d'une même plante. C'est ainsi qu'un grand nombre de végétaux inférieurs, appartenant au groupe des Cryptogames (Lichens, Algues, C'hampignons, Mousses) sont exclusivement composés de cellules et méritent le nom de végétaux cellulaires, qu'on leur donne souvent, par opposition aux autres Cryptogames (Fougères, Prêles, etc.) et aux Phanérogames qui offrent, outre le tissu cellulaire, des tissus fibreux et vasculaires. Ces derniers même y prédominent notablement, le premier ne se rencontrant que dans les parties jeunes des plantes. 


\section{CHAPITRE II}

\section{Substances diverses contenues dans les cellules végétales.}

Classitication des produits cellulaires. Amidon; Inulne; Dextrine; Chlorophylle; Substances albuminoïdes cristallisées, Aleurone; Matières grasses; Matières sucrées; Tannin; Matières colorantes; Matières gommeuses; Concrétions minérales solides; Gaz. Organes sécréteurs et Sécrétions proprement dites: Essences, Résines, etc.; Latex.

Classification des produits cellulaires. - Les cellules des végétaux renferment outre le protoplasma un grand nombre de substances qui sont dues à son activité ; la plupart sont des matériaux utiles, des substances nutritives qui seront utilisées au fur et à mesure des besoins de la plante, ou des réserves qui seront employées à un moment donné; les autres ne paraissent pas avoir une grande importance dans la vie de la plante, mais peuvent être regardées plutôt comme des produits de désassimilation, produits qui cependant pourront, par suite de modifications chimiques accomplies dans l'intérieur même des tissus, concourir de nouveau à la nutrition.

Parmi les premiers, nous étudierons l'amidon, l'inuline, la dextrine, la chlorophylle, les matières albuminoïdes cristallisées, l'aleurone, les matières grasses, sucrées, le tannin; parmi les secondes nous passerons en revue les matières colorantes autres que la chlorophylle, puis les matières gommeuses, les concrétions minérales solides, les sels, les acides, les gaz.

Un paragraphe spécial sera consacré aux Sécrétions proprement dites, essences, résines, etc., et aux organes qui 
qui les produisent. Enfin nous terminerons cette étude par celle du latex, renfermé dans les vaisseaux laticifères.

Sa répartition dans les végétaux.

Amidon. - De toutes les substances résultant du travail accompli dans les cellules, l'amidon ou fécule est la plus abondante et l'une des plus importantes pour la vie du végétal. La plupart des plantes en renferment et souvent en quantité si considérable dans leur tige, dans leur racine, dans leurs feuilles ou leurs graines, qu'elles entrent à ce titre dans le régime de l'homme et des animaux, ou bien qu'on peut l'en extraire pour les usages industriels ou alimentaires. Citons, parmi les plus importants, le Blé, le Seigle, l'Avoine et les autres céréales; beaucoup de graines de Légumineuses, haricots, lentilles, etc., certains Palmiers, qui donnent l'arrow-root et le tapioca, la Pomme de terre, etc., etc.

Caractères clii. C'est un hydrate de carbone $\left(\mathrm{C}^{12} \mathrm{H}^{10} \mathrm{O}^{10}\right)$, dont la constimiques.

tution élémentaire est par conséquent semblable à celle de la cellulose. Il offre avec la teinture d'iode une réaction caractéristique, consistant dans la couleur bleue ou violette qu'il prend alors ; le chlorure de zinc lui donne la même teinte, mais en altérant bientôt la forme des grains.

Il faut ajouter encore que chaque grain d'amidon est formé dedeux substances intimement mêlées, mais parfaitementisolables et qu'on appelle la granulose et l'amylose. La première est soluble dans la salive et se colore en bleu par l'iode; l'autre est insoluble dans la salive et se colore en jaune ou ne se colore pas par l'iode. Cette dernière constitue en quelque façon le squelette du grain d'amidon, de sorte que la granulose en étant enlevée, bien qu'elle soit en beaucoup plus forte proportion, le grain conserve sa forme et son apparence primitive. Lamylose ne constitue que $60 / 0$ de l'amidon de la pomme de terre et seulement $20 / 0$ de celui du blé.

Caractères phy. L'amidon est sous forme de grains d'une extrême petitesse, siques. d'aspect variable, arrondis, ovoïdes, discoïdes, ellipsoïdes, etc., mais assez constant dans une même espèce de plantes et assez nettement tranché de l'une à l'autre, pour qu'il soit possible, le plus souvent, de les distinguer entre eux à l'aide 
du microscope; aussi n'est-il rien de plus facile que de reconnaître les fraudes commerciales qui consistent á mêler à la farine du froment celle de haricots, de fèves ou de pommes de terre.

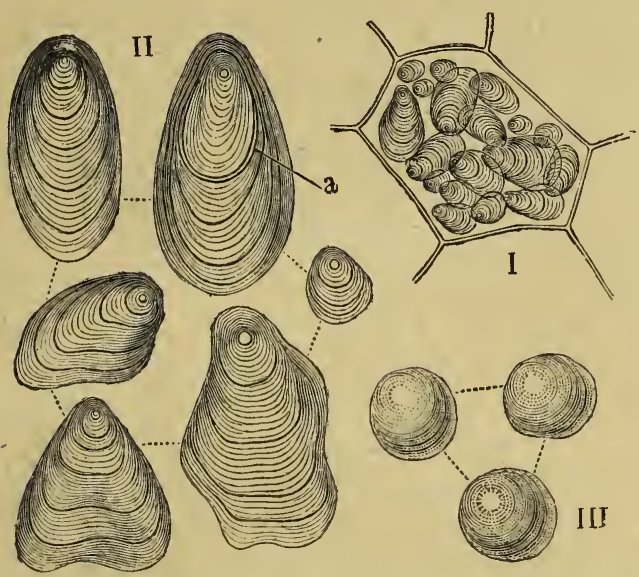

Fig. 25. - Grains d'amidon; I, une cellule du parenchyme de la ponme de terre, grossie 150 fois, renfermant des grains d'amidon; II, ceux-ci plus grossis montrant nettement leurs zones concentriques $(a)$, (gross. 600 fois); III, grains d'amidon du blé, (gross. 600 fois).

Vu au microscope, chaque grain paraît formé d'un certain nombre de couches concentriques disposées autour d'une partie plus sombre, le hile, appelé ainsi parce qu'il ressemble à une petite cicatrice, que l'on croyait jadis être son point d'altache. Le hile peut être placé au centre du grain, de telle sorte que les zones soient régulièrement disposées autour de lui, ou bien en un point excentrique et alors ces dernières sont beaucoup plus nombreuses d'un côté que de l'autre. Sa forme est celle d'un point quand le grain est à peu près arrondi, mais prend l'apparence d'une fente quand il est allongé.

La disposition zonaire des grains d'amidon n'est pas due, comme on pourrait le croire, à des dépôts successifs; c'est un phénomène qui se produit secondairement et qui tient à ce que la petite masse se dispose en couches alternativement plus denses et moins denses par suite d'une 
différence dans la proportion d'eau qu'elles renferment. Toutefois, les recherches les plus récentes tendent à prouver que l'accroissement des grains d'amidon ne se fait pas par intussusception, mais par des dépôts à la surface extérieure, comme se fait l'accroissement des cristaux inorganiques. Bien plus, on démontre que chaque grain n'est en somme que l'agglomération d'une grande quantité de cristaux prismatiques disposés en rayons.

Les grains d'amidon ne se présentent pas toujours à ce degré de simplicité : dans certaines plantes on les trouve accolés plusieurs ensemble; l'amidon du Manioc, si employé dans notre alimentation sous le nom de tapioca, en fournit un excellent exemple; quand il s'offre à cet élat on le dit agrégé. Dans d'autres cas, les grains commencent par être simples, mais par suite de leur division en 2,4 ou un plus grand nombre de parties qui restent soudées les unes aux autres, chaque grain paraît formé de plusieurs intimement unis entre eux, et on dit alors que les grains sont composés.

Mode de formation.

Rôle de lamidon.

Quant au mode de formation de l'amidon, on n'est pas complètement fixé à son sujet. On sait toutefois qu'il apparaît sous forme de fines granulations dans le proloplasma, et notamment dans les petits corps ou leucites qui constituent des grains de chlorophylle, dont l'étude va bientôt nous occuper; aussi est-on autorisé à croire que le rôle de celle-ci est considérable dans l'élaboration de l'amidon, ct même que c'est là une de ses principales fonctions; toutefois il n'y a pas que les leucites chlorophylliens à produire l'amidon, celui-ci se formant aussi dans des leucites spéciaux qui paraissent n'avoir pas d'autre fonction que de travailler à son élaboration.

L'amidon, si abondamment répandu dans les plantes, joue un rôle considérable au point de vue de leur nutrition. Il est insoluble dans l'eau, il est vrai, celle-ci ne faisant autre chose que de le gonfler et de le ramollir, de sorte qu'il se prend alors en une masse visqueuse, transparente, bien connue en économie domestique sous le nom d'empois. Mais la plante produit un principe particulier, un ferment, la diastase, qui a le pouvoir de modifier l'amidon et 
de le transformer en dextrine, puis en glycose, état sous lequel il est soluble et susceptible alors d'être transporté dans les différentes parties de la plante pour servir à sa nutrition actuelle, ou bien pour s'accumuler dans certaines régions afin d'y constituer des réserves nutritives. (Voy. Chap. de la Digestion, Réserves nutritives.)

Inuline. - Son nom vient de l'Aunée (Inula Helenium), plante de la famille des Composées, où elle est très abon-

Sa répartition dans les dante; d'ailleurs la plupart des plantes de cette famille en présentent; les racines de Dahlia, de Topinambour en sont particulièrement riches; on la trouve encore chez les végétaux de plusieurs familles voisines de celle-ci, et aussi dans des Algues, des Champignons, etc. Elle parait suppléer l'amidon, si bien que celui-ci fait défaut dans les plantes où existe l'inuline.

C'est, comme l'amidon, une sub-

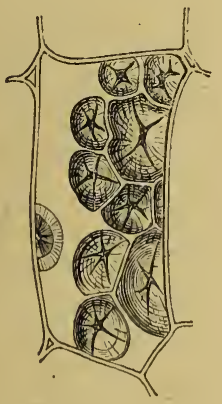

Fig. 26. - Inuline en cristaux sphériques dans une cellule de la racine de Dahlia, gross. 500 fois. stance ternaire et de même compo-

Composition chimique. sition chimique $\left(\mathrm{C}^{12} \mathrm{H}^{10} \mathrm{O}^{10}\right)$, mais qui en diffère à d'autres points de vue.

Insoluble dans l'eau froide, l'inuline se dissout très bien dans l'eau chaude; l'iode ne la colore pas en bleu comme l'amidon.

Contrairement à l'amidon elle est Caractìres phy. soluble dans le suc cellulaire, et c'est siques. à l'état de solution qu'elle se trouve dans la plante vivante; mais si les organes de la plante se dessèchent, ou bien, si on en recueille les sucs poul les faire évaporer, on observe l'inuline à l'état solide, et sous forme soit de granulations, soit de petites masses cristallisées, à peu près sphériques, composées chacune d'un grand nombre de petits cristaux prismatiques rayonnant d'un centre commun.

Chose curieuse, un même cristal peut se former aux dépens de l'inuline renfermée dans plusieurs cellules contiguës, et se trouver moitié dans une cellule et moitié dans 
l'autre; de sorte que la cristallisation de cette substance n'est nullement empêchée par l'interposition des membranes cellulaires.

dextrine. - Nous ne dirons qu'un mot de la dextrine, parce qu'elle dérive de l'amidon, dont il a été longuement question un peu plus haut; elle a une importance considérable, puisque c'est sous cet état que l'amidon chemine à travers les tissus. Très abondamment répandue dans les plantes, elle est à peu près incolore, transparente et visqueuse; sa formule est celle de l'amidon $\left(\mathrm{C}^{12} \mathrm{H}^{10} \mathrm{O}^{10}\right)$; son nom vient de ce qu'elle dévic à droite la lumière polarisée.

Répartition de la chlorophylle.
Chlorophylle. - Matière verte très répandue dans les végétaux et dont le rôle est de la plus haute importance(Voy. Chap. VII, Fonction chlorophyllienne); elle fait cependant défaul dans toute une classe du règne végétal, celle des Champignons. D'autres plantes paraissent en manquer, par exemple, les Algues rouges ; mais ce n'est alor's qu'une apparence, la couleur de la chlorophylle étant simplement masquée par une autre teínte; il en est de mème pour les feuilles de certaines plantes phanérogames, dont les cellules superficielles sont colorées par un pigment rouge ou jaune, la chlorophylle étant renfermée dans les cellules plus profondes. Chose remarquable, les plantes ne sont pas les seuls êtres organisés qui présentent de la chlorophylle; on connaît parmi les animaux inférieurs plusieurs espèces, telles que des Infusoires, des Vers, qui en contiennent également et chez lesquels la chlorophylle joue le même rôle que nous lui voyons remplir dans la Plante. Ce n'est donc pas dans la présence ou labsence de cet élément qu'il faut chercher un indice certain de séparation entre les deux règnes organiques.

Origine de la chlorophyile.

La chlorophylle se forme dans le protoplasma cellulaire et toujours sous l'influence de la lumière solaire. Toute plante qui est privée de l'action de celte dernière s'étiole, c'est-à-dire que la matière verte ne s'y développe pas et que celle qui y existait se détruit. Cependant il existe par- 
fois des parties colorées en vert qui n'ont jamais subi l'action de la lumière, telles que les embryons de quelques plantes qui, encore renfermés dans la graine, offrent déjà celte coloration, résultat de la présence d'une petite quantité de chlorophylle.

D'autre part, il faut faire remarquer que, sous l'influence d'une lumière trop intense la chlorophylle disparaît également, et que la plante s'étiole.

La chlorophylle est en réalité essentiellement formée de protoplasma dans lequel elle apparaît sous forme de petits

Nature de la chlorophylle. corpuscules blancs ou incolores appelés leucites ( $\lambda \varepsilon \tilde{x}$ xos, blanc), qui prennent d'abord et indépendamment de la
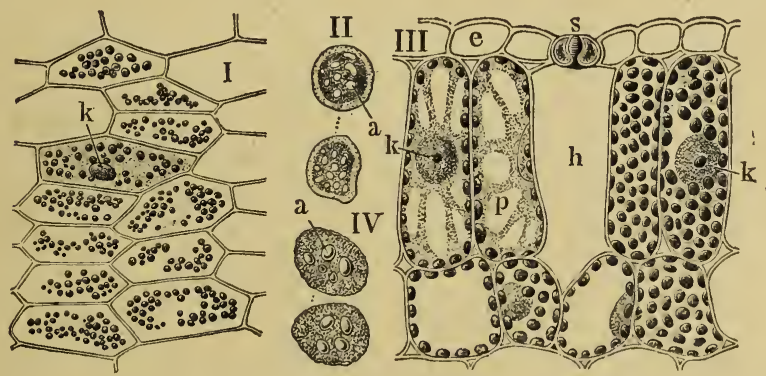

Fig. 2\%. - Cellules avec grains de chlorophylle; I, cellules d'une feuille de Marchantia polymorpha; $k$, noyau cellulaire (gross. 200 fois); II, les grains de chlorophylle des mêmes cellules renfermant des grains d'amidon (a), (gross. 900 fois); III, coupe a travers une feuille de Five, dont les cellules sont remplies de grains de chlorophylle; $k$, noyau cellulaire; $p$, protoplasma; $e$, épiderme; $s$, stomate $h$, chambre stomatique.

lumière une teinte jaune due à un principe spécial, la

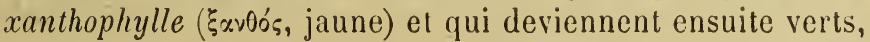
mais seulement par suite de leur exposition à la lumière, laquelle provoque la formation d'un second principe colorant qui est la chlorophylle proprement dite.

Il résulte de ceci que les corps chlorophylliens ou les grains de chlorophylle, comme on les appelle souvent, existent en réalité dans les plantes placées à l'obscurité, la teinte verte seule leur faisant défaut.

Au lieu de se présenter', comme c'est la règle, à l'état de granules, la chlorophylle peut exister sous forme de long.s 
rubans disposés en spirale, comme on le voit dans les fig. 18 et 28; il en est ainsi dans bon nombre d'Algues inféricures.

La chlorophylle ct lamidon.

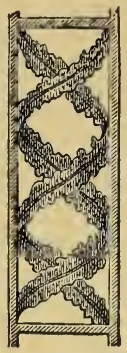

Fig. 28. - Chlorophylle en rubans spiraux dans une rellule d'Algue (Spirogyra) (gross. 300).

La chlorophylle étant soluble dans l'alcool et dans la benzine, en traitant par ces deux réactifs des plantes riches en chlorophylle, préalablement broyées, on obtient une solution qui par l'évaporation du liquide laisse cristalliser la chlorophylle sous forme de petites aiguilles rayonnées.

Entre la chlorophylle et l'amidon il existe d'importantes relations. Si la cellule dans laquelle la matière colorante se développe ne contient pas d'amidon, elle apparaît brusquement par place; si elle en contient, c'est autour des grains de celui-ci qu'elle apparaît.

D’un autre côté, dans les grains de chlorophylle se forment presque toujours des grains d'amidon placés soit vers son centre, soit dans sa couche périphérique; les grains d'amidon grossissent de plus en plus, de sorte qu'il arrive souvent, surtout quand il y en a plusieurs, qu'ils absorbent tout le corpuscule chlorophyllien qui disparaît complètement; mais à partir de ce moment, les grains d'amidon ne peuvent plus s'accroître.

Quant à l'accroissement des grains de chlorophylle, il a lieu par intussusception; et quand ils sont arrivés à un certain volume, ils s'étranglent et se divisent en deux leucites nouveaux.

Au moment où la végétation se ralentit, les grains de chlorophylle éprouvent d'importants changements; ainsi à l'automne, au moment où les feuilles vont tomber, ils se dissolvent dans le protoplasma, abandonnent les parties qui meurent et se portent vers celles qui continuent ì vivre.

Substances albuminoïdes cristallisées; Aleurone. Il est curieux de rencontrer dans les plantes des corps de 
nature albuminoïde à l'état cristallisé, c'est-à-dire ayant les caractères géométriques et optiques et présentant les clivages des cristaux ordinaires, mais qui en diffèrent en ce que, au lieu d'être de structure homogène, ils sont formés de couches emboîtées les unes dans les autres, alternativement plus dures et plus molles, la plus externe étant toujours la plus dure, l'interne, la plus molle; ces particularités tiennent à une proportion d'eau différente d'une zone à l'autre.

Cette propriété de se présenter sous une forme cristallisée, a valu à ces corps le nom de cristallö̈des, mais il faut bien remarquer qu'ils ne sont pas tous identiques, et que la façon différente dont ils se comportent avec les réactifs montre que l'on doit les rapporter à des principes albuminoïdes distincts.

Les cristalloïdes sont tantôt complètement libres dans les cellules, tantôt enclavés dans certaines substances qui sont des matériaux de réserve et auxquelles on donne le nom d'aleurone.

Les graines oléagineuses, notamment celles du Ricin, sont favorables à l'étude de cette substance. Elle est très répandue Répartition dans les végétaux, parfois même plus abondante que l'amidon, surtout dans les graines huileuses.

Elle se présente sous la forme de grains ovoïdes, incolores ou grisâtres, ou di.
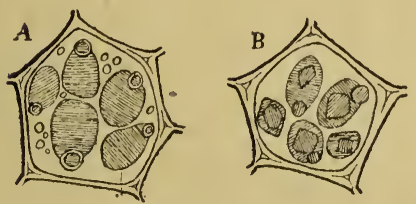

Fig. 29. - Cellules très grossies de la graine du Ricin, renfermant de l'aleurone; A, chaque grain d'aleurone montre seulement un globoïde (préparation dans la glycérine), et en $B$, un globoïde et un cristaloïde (préparation chauffée dans la glycérine), (d'après SACHS). versement colorés dan certaines plantes. Les uns ne se composent pas d'autre chose que de protoplasma amorphe; mais les autres ont une structure complexe et fort singulière. Sous une couche de protoplasma amorphe se trouve un cristalloïde de nature albuminoïde, qui occupe la partie la plus renflée du grain d'aleurone, tandis que l'autre extrémité renferme un corps arrondi qu'on appelle le globoïde. En somme ces deux parties, le cris- 
talloïde et le globoïde, doivent être regardées comme des enclaves accessoires des grains d'aleurone, car l'un ou l'autre peut manquer, ils peuvent même faire défaut tous les deux, comme on l'a vu tout à l'heure. Ajoutons que les grains d'aleurone renferment parfois des cristaux d'oxalate de chaux.

Leurs réactions chimiques comme leur structure diffèrent de celles de l'amidon; ainsi la teinture d'iode les colore en brun-jaunâtre, l'azotate acide de mercure en rouge-brique, réactions caractéristiques des substances albuminoïdes.

Origine de l'aleurone.

Leur formation et leur rôle sont assez mal connus; on croit qu'ils dérivent de l'amidon; en tous cas, ils n'apparaissent dans la graine qu'au moment de sa complète maturité, alors qu'elle commence à se dessécher; leur formation correspond donc à la perte d'une certaine quantité d'eau. Quand les grains renferment un cristalloïde et un globoïde, ceux-ci apparaissent en même temps, mais à an degré d'extrême petilesse, puis s'accroissent ensuite et s'entourent d'une couche amorphe qui est l'aleurone proprement dite.

Rôle Au moment où la graine va germer, l'aleurone se dissout de l'aleurone. dans le suc cellalaire et se résorbe lentement pour fournir à l'alimentation de la plantule. Sa formation correspond par conséquent à celte période dans laquelle la plante passe de la vie active à la vie latente, et son emploi à la période dans laquelle la plante passe au contraire de la vie latente it la vie active.

On doit donc considérer l'aleurone comme un aliment de réserve, au même titre que l'amidon; mais tandis que celui-ci est un hydrate de carbone, une substance ternaire, l'autre est un aliment quaternaire, de nature albuminoïde.

Leur répartition.

Matières Grasses. - Après l'amidon les corps gras constituent les substances ternaires les plus importantes et les plus répandues. Souvent à l'état liquide ou d'huile, ils se trouvent, dans d'autres cas, à des degrés de consistance variable, qui leur ont valu, comme aux produits similaires du règne animal, les noms de beurre, de suif et de cire. 
Presque toujours le contenu cellulaire présente une certaine proportion de substance grasse, de consistance huileuse, sous forme de goultelettes arrondies, que leur réfringence permet de reconnaître au premier abord.

De plus, certains organes sont gorgés de matières grasses en telle quantité que l'on peut les extraire avec avantage, soit pour l'alimentation, soit pour divers usages industriels. Ces organes sont assez variables suivant les plantes; en gé néral cependant ce sont les fruits, soit la partic charnue ou péricarpe, soit la graine, ce qui est le cas de beaucoup le plus fréquent.

Ainsi les fruits ou les graines d'Olivier, de Noyer, de Noisetier, de Golza, de Navette, d'Amandier, d'Arachide, etc., contiennent une forte proportion d'huile, que l'on extrait dans un but alimentaire ou industriel.

Un arbre qui croît en Chine, le Gluttier à suif, donne un produit qui sert à fabriquer des chandelles très employées en Chine et au Japon.

Les fruits du Cocotier, du Cacaoyer, du Muscadier et plusieurs autres donnent un beurre comestible ou médicinal.

La cire est produite à la surface des feuilles d'un grand nombre de plantes et s'y dépose sous forme de granules, de bâtonnets ou de lamelles; certains arbres de l'Amérique du sud, les Palmier's à cire, en produisent une assez grande quantité pour qu'elle soit recueillie avec avantage et employée aux mêmes usages que la cire d'abeilles. Nombre de plantes de notre pays offrent une mince couche cireuse à la surface de leurs feuilles, qui empêche l'eau d'y adhérer' et de les mouiller: telles sont entre autres celles du Chou, de la Capucine, du Rosier, etc. D'autres fois c'est à la sur'face des fruits que la cire se rencontre, notamment sur les prunes, el surtout ceux du Myrica cerifera, plante de la Louisiane. Enfin c'est parfois la tige elle-mème qui ell est recouverte, celle de la Canne à sucre, par exemple.

Parni les substances grasses que produisent les plantes, les unes n'ont aucune utilité pour leur nutrition, mais cons. Rôle des matières grasses. tituent des moyens de protection, et peuvent être regardées 
comme des matériaux à éliminer; les autres, au contraire, notamment celles qui sont accumulées dans les graines, sont destinées à jouer, dans la nutrition du végétal, un rôle important. A l'époque de la germination, sous l'influence d'une substance azotée produite par le protoplasma, le ferment émulsif, elles sont émulsionnées, puis saponifiées, c'est-ìdire dédoublées en un acide gras et en glycérine, lesquels, à leur tour, s'oxydent et subissent des modifications diverses, pour produile, en fin de compte, des hydrates de carbone et notamment de l'amidon.

Enfin, il paraît hors de doute qu'une portion des substances grasses est brûlée lentement dans l'organisme végétal et se transforme en eau et acide carbonique, en produisant une certaine quantité de chaleur. (Voy. Ghap vir, Respiration.)

Matières sucrées. - Très abondamment répandues dans les cellules végétales, à l'étal de solution dans le suc cellulaire et contribuant à la nutrition actuelle, ou bien accumulées dans certains organes pour servir de réserves nutritives, les matières sucrées se présentent sous trois formes, de composition chimique et de propriétés physiques différentes, à savoir la Glycose, la Saccharose et la Mannite.

Glycose. La glycose $\left(\mathrm{C}^{12} \mathrm{H}^{12} \mathrm{O}^{12}\right)$ offre surtout deux variétés bien connues: la glycose ordinaire ou sucre de raisin ou dextrose, qui dévie à droite le plan de polarisation de la lumière, est très soluble dans l'eau, où elle forme, à la suite d'un repos prolongé, des cristaux groupés en masses mamelonnées. Elle doit sa formation au dédoublement de la saccharose par le ferment inversif ou à l'hydratation de la maltose. La lévulose, qui abonde dans les fruits mûrs acides, dévie à gauche la lumière polarisée, cristallise en aiguilles et est due également au dédoublement de la saccharose par le ferment inversif ou encore à l'hydratation de l'inuline.

On connaît encore d'autres glycoses : la sorbine dans les baies du Sorbier, les fleur's de l'Amandier, du Co- 
gnassier, etc.; l'inosine, dans les fruits du Haricol, les feuilles du Noyer, du Chou, etc.

La saccharose ou sucre de canne $\left(\mathrm{C}^{24} \mathrm{H}^{22} \mathrm{O}^{22}\right)$, très abondante dans la Canne à sucre, les racines de Betterave, de Carotte, la tige de l'Érable, les graines du Châtaignier, etc., est très soluble dans l'eau, et cristallise en prismes rhomboïdaux obliques; sa solution dévie à droite le plan de la lumière polarisée.

Sous l'influence du ferment inversif, il prend deux équivalents d'eau el se dédouble en glycose et lévulose, dont le mélange porte le nom de sucre interverti, parce qu'il dévie non plus à droite mais à gauche la lumière polarisée.

Il existe plusieurs variétés de saccharose : la maltose, qui résulte du dédoublement de l'amidon par la diastase; la synanthrose, très répandue dans les plantes de la famille des Composées, et qui existe dans les graines du Seigle, tandis que les autres céréales renferment du sucre de canne; la mélitose, qui se trouve dans l'Eucalyptus, etc.

La mannite $\left(\mathrm{G}^{12} \mathrm{H}^{14} \mathrm{O}^{12}\right)$ est un hydrate de carbone renMannite. fermant de l'hydrogène en excès. Elle existe abondamment dans l'Érable, le Frêne, l'Olivier, le Céleri, etc., plusieurs Algues (Laminaria saccharina). Soluble dans l'eau, elle cristallise en prismes rhomboïdaux droits, très fins, soyeux, souvent rayonnés. Sa solution dévie légèrement à gauche le plan de la lumière polarisée. On connaît un assez grand nombre d'espèces de mannite, dont l'énumération serait icí peu utile.

Matières tanniques. - Le tannin $\left(\mathrm{G}^{14} \mathrm{H}^{10} 0^{9}\right)$ est une substance acide voisine des glycoses, très généralement répandue dans les régétaux, soit à l'état de dissolution dans le suc cellulaire, soit accumulée dans certaines cellules, comme celles de l'écorce du Chêne, surtout des jeunes rameaux. Il existe dans nombre de feuilles, de fleur's, de fruits, surtout avant leur maturité; c'est dans les galles de Chêne, vulgairement connues sous le nom de noix de galle, qu'il est le plus abondant, sa proportion allant jusqu'à $26 \%$.

Il offre des réactions caractéristiques avec les sels de fer, 
qui lui donnent une coloration bleue ou noire, d'où son emploi dans la fabrication de l'encre; il jouit de la propriélé de se combiner avec les substances arimales en les rendant imputrescibles, ce qui en fait une substance précieuse pour le tannage; enfin il coagule les solutions de gélatine et de matière albuminoïde, propriété qui le fait encore utiliser dans plusieurs industries.

Au point de vue de la physiologie de la plante, on doit le regarder comme un aliment de réserve susceptible de se transformer en amidon ou en glycose, par exemple, dans les fruits qui arrivent à leur maturité.

Matières colorantes autres que la chlorophylle (1). Des matières colorantes autres que la chlorophylle se rencontrent dans divers organes des végétaux. Leur lieu d'élection est surtout les pétales des fleurs et parfois les feuilles ou les frondes des Cryptogames. Le groupe des Floridées, parmi les Algues, nous en fournit de remarquables exemples. On y voit souvent, en effet, des corpuscules ou leucites colorés en rouge ou en brun, parfois en bleu ou en jaune, et assez abondants pour masquer la chlorophylle.

Mais la coloration ne dépend pas toujours de la présence de corpuscules solides; ainsi qu'on le dira dans un autre chapitre, des liquides ou des gaz peuvent en être la véritable cause. (Voy. Ghap. vir. Structure du Calice et de la Corolle.)

Matières gommeuses. - Le suc cellulaire d'un certain nombre de plantes renferme une matière épaisse, mucilagineuse ou gommeuse, qui offre une grande analogie avcc la dextrine; les racines de Guimauve, de Consoude, de Saponaire en sont surtout abondamment pourvues. Cette substance semble être parfois le résultat d'une élaboration accomplie par le protoplasma; mais peut-être aussi n'est-clle que

(1) Ce sujet devant revenir à l'occasion de la coloration des fleurs, sera traité brièvement ici. 
le fait d'une altération, d'une gélification de la paroi interne de la membrane cellulaire, altération qui prend des proportions considérables dans les plantes qui donnent les différentes sortes de gommes arabique, adragante, ou autres, employées à plusieurs usages, et celle que nous voyons s'écouler à l'extérieur par les crevasses de nos arbres fruitiers. Ces derniers produits paraissent constamment dus à de véritables maladies, ayant pour effet de ramollir et de détruire les parois des cellules et de les transformer en une sorte de gelée qui finit par se faire jour au dehors.

Un phénomène analogue se produit quand on humecte des graines de lin ou de coing : les cellules extérieures absorbent l'eau avidement, se distendent, se déchirent et se transforment en un mucilage.

Concrétions minérales solides. - Le suc cellulaire tient souvent en dissolution une certaine proportign de sels, qui ont presque toujours la chaux pour base. Ils sont parfois en si grande abondance qu'ils se précipitent sous forme de cristaux ou de concrétions.

Les principaux sont l'oxalate, le carbonate, le tartrate, le sulfate de chaux; mais le premier est de beaucoup le plus commun.

Les cristaux d'oxalate de chaux (fig. $30, I I$ i $V$ ) se présentent parfois sous forme d'aiguilles, réunies en paquets ou faisceaux et désignées sous le nom de raphides, disposition fréquente chez les Monocotylédones, où l'on voit certaines cellules qui en sont presque entièrement remplies.

D’autre fois, leur forme appartient au système clinorhombique ou au système quadratique. Souvent ils sont pour ainsi dire encastrés les uns dans les autres, pour former des mâcles (fig. 30, III), dont la masse remplit presque entièrement la cavité cellulaire et se trouve souvent enveloppée de protoplasma ou de cellulose en continuité avec les parois de la cellule, comme dans la fig. $30, V$.

Le carbonate de chaux (fig. $30, I$ ) est ordinairement à l'état de concrétions amorphes, connues sous le nom de 


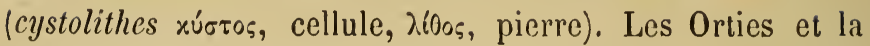
plupart des plantes de la famille des Urticées en présentent constamment et de forme très variable. Celles des feuilles
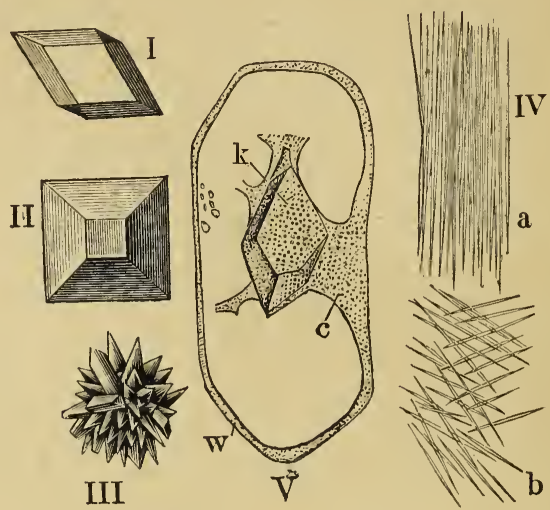

Fig. 30. - Cristaux des cellules végétales; I, cristal rhomboèdrique de carbonate de chaux; II à V, cristaux d'oxalate de chaux : II dans le système quadratique; III, en mâcles, pris dans un nectaire de Mauve (gross. 600); IV, $a, b$, raphides de feuilles de Fuchsia (gross. 450); V, cellule du fruit du Rosier avec un cristal $(k)$ soutenu au milieu de la cellule $(w)$ par un prolongement $(c)$ du protoplasma.

de la plante ornementale connue sous le nom de Caoutchouc (Ficus elastica) sont particulièrement remarquables. On dirait un petit lustre attaché par un fin cordon de cellulose à la voûte de la cellule. Le microscope montre que ces concrétions sont composées de cellulose mêlée de grains calcaires.

Silice. La silice se rencontre à l'état de concrétions dans les tissus d'un assez grand nombre de végétaux, notamment dans les Prêles, où son abondance dans les parties superficielles de la tige fait employer ces plantes au polissage du bois et des métaux; dans les tiges de Bambous où elle forme des concrétions connues sous le nom de tabaschir; dans les Rotangs, vulgairement appelés Rotins, qui lui doivent leur poli et leur dureté. Citons, pour terminer, les 
Algues du groupe des Diatomées, entièrement recouvertes d'une carapace siliceuse.

GAZ. - Le suc cellulaire tient toujours en dissolution certains principes gazeux, notamment de l'air et de l'acide carbonique, que les racines ou les feuilles ont puisé dans l'air ou dans l'eau. Ces gaz circulent également en dehors des cellules, dans les méats intercellulaires; mais on les trouve surtout dans les cellules dont le protoplasma a disparu. On verra dans un autre chapitre que les vaisseaux du bois sont surtout réservés à la circulation des gaz.

Organes sécréteurs et Sécrétions végétales.

Ce qu'Il faut entendre par sécrétio:s véGétales. Dans le sens le plus général, on pourrait définir les sécrétions : "la formation de toutes les substances élaborées par le protoplasma ", et faire rentrer sous ce titre aussi bien la production de l'amidon et de la chlorophylle, par exemple, que la formation et le dégagement de la vapeur d'eau et de l'acide carbonique.

Mais en se plaçant à un point de vue plus restreint, on réserve ce nom aux fonctions de certains organes affectant souvent une forme particulière et qu'on appelle les organes sécréteurs ou glandes.

Quant à une distinction entre les sécrétions et les excrétions, elle n'a qu'une faible importance physiologique et consiste en ce que, dans le premier cas, le produit sécrété reste enfermé dans les tissus de la plante, tandis que dans le second, il se fait jour à l'extérieur.

Les produits des sécrétions végétales doivent être tous regardés comme excrémentitiels et naturellement étrangers à la nutrition; toutefois, ils peuvent, du moins quelques- 
uns, être repris par les cellules voisines et rentrer dans la circulation générale.

Glandes superficielles.

Organes sécréteurs. - Ils ont la forme, la structure et la situation les plus variées. Et d'abord, les uns sont superficiels, les autres profonds. Les premiers sont silués dans les
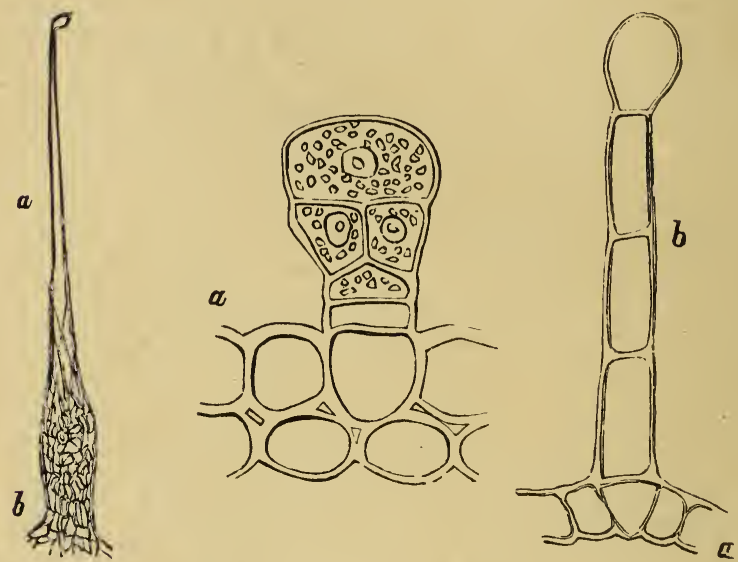

Fig. 31. - La fig. de gauche représente un des poils urticants de l'Ortie; $a$, tige du poil ; $b$, sa base en forme de bourrelet. - La figure suivante représente un poil sécréteur du pétiole d'une feuille de l'Ortie blanche, portés par les cellules de l'épiderme. - La figure de droite montre un poil d'une tige de Géranium; $a$, cellules épidermiques; $b$, poil.

cellules de l'épiderme ou dans des cellules prolongées en forme de poils qui en dépendent; tel est le cas de l'Ortie commune, dont les poils terminés par une pointe aiguë, renferment dans leur cavité centrale un liquide très irritant qui s'épanche dans la petite piqûre faite par elle et dans laquelle elle se brise; tel est encore celui de nombre de Labiées, dont les poils épidermiques renferment souvent des liquides odorants; dans l'Ortie blanche, chaque poil est renflé à son extrémité el renferme une glande formée de plusieurs cellules. Il faut faire rentrer dans cette catégorie les glandes du stigmate du pistil et des jeunes bourgeons, qui laissent, les uns et les autres, échapper un liquide 
visqueux, et encore les glandes pédiculées si répandues à la surface des feuilles des plantes dites carnivores. (Voy. Chap. vir. Digestion.)

Les glandes profondes, situées dans l'épaisseur des feuilles, des organes floraux, de la tige ou des fruits, sont extrêmement variables de forme et de structure.

Les unes sont formées d'une cellule unique, mais plus souvent d'un petit groupe de cellules, dans l'intérieur desquelles s'accumule le produit élaboré ; souvent ces cellules éclatent et se déchirent, el il en résulte une petite cavité commune remplie du liquide sécrété, comme c'est le cas

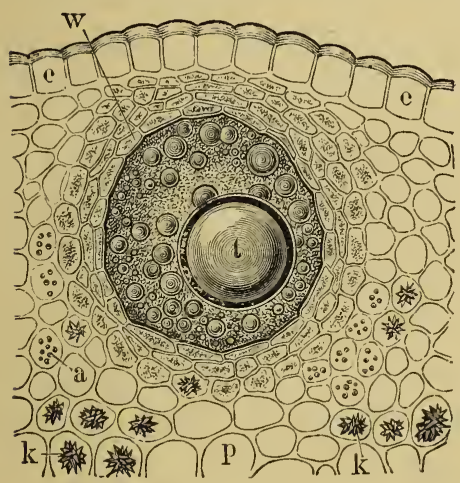

Fig. 32. - Glande huileuse de l'ovaire du Metrosideros buxifolia coupé en travers et grossi $300 \mathrm{f}$; $e$, épiderme; $p$, parenchyme; $w$, paroi interne de la glande; $t$, grosse goutte d'huile; $k$, cristaux; $a$, grains d'amidon. des glandes des fruits du Citronnier, de l'Oranger. D'autres fois, les cellules sécrétantes sont disposées autour d'une cavité centrale ou petite poche, qui peut être arrondie, comme c'est le csa des Conifères, ou allongée en forme d'un petit canal, comme cela se voit dans le fruil des Ombellifères, où les glandes ont reçu pour cette raison le nom de bandelettes. Les cellules glandulaires ont elles-mêmes une forme allongée, et sont disposées en files superposées; les cloisons intermédiaires se résorbent; on a alors de véritables canaux sécréteurs, qui conduisent par des transitions insensibles jusqu'à la structure des vaisseaux laticifères.

Le plus souvent, le produit de sécrétion resle enfermé dans les tissus et ne sort pas à l'extérieur, mais il y a un assez grand nombre d'exceptions; ainsi les glandes stigmatiques du pistil, celles des bourgeons, laissent échapper un liquide visqueux qui, dans le premier cas, relient

Glandes profondes 
les grains de pollen, et dans le second cas sert de vernis protecteur; les nectaires, dont la plupart des fleurs sont pourvues, laissent écouler leur sécrétion au fond de cellesci, où elle attire les insectes; de même, les glandes des plantes dites carnivores laissent exsuder un liquide qui s'accumule comme une petite perle à leur' extrémité. Tantôt la sortie de la sécrétion se fait par simple exhalation à travers la paroi cellulaire et tantôt par déchirure ou destruction de celle-ci.

Produits de la sÉcrétion des glandes. - Les organes sécréteurs qui viennent d'être étudiés donnent naissance à des produits très divers, le plus souvent liquides, quelquefois solides, presque toujours aromatiques et chimiquement formés de carbone et d'hydrogène. Mais la plupart d'entre eux, aussitôt formés, prennent de l'oxygène et absorbent de l'eau, de sorte que la combinaison primitivement binaire devient ternaire: le carbure d'hydrogène devient un hydrate de carbone. Ces produits portent le nom d'essences, d'oléorésines, de résines, de gommes-résines et de baumes.

Essences. Les essences n'ont aucun rapport, malgré les noms d'huiles volatiles, d'huiles essentielles qu'on leur donne souvent, avec les substances grasses. Toutes sont liquides à la température ordinaire, à une exception près, le Camphre. Elles résultent du mélange de deux principes, ou si l'on veut, de deux huiles essentielles distinctes, dont l'une est cristallisable (stéaroptène) et dont l'autre reste toujours liquide (éléoptène). A la longue, elles s'altèrent à l'air et à la lumière, s'oxydent et se résinifient. Citons parmi les principales essences : celles de Rose, de Laurier, le camphre du Japon, qui se forment dans le parenchyme des feuilles; celles de Thym, d'Hysope et d'un grand nombre de Labiées, qui se forment à l'intéricur de cellules très superficielles ou de poils épidermiques; les essences d'Anis, de Coriandre, de Fenouil, fournies par les fruits de ces plantes; celles de Cannelle, le camphre de Bornéo, élaboré dans la tige et les rameaux.

On les obtient par pression ou distillation; quelques-unes 
ne se forment que par la trituration des organes qui les contiennent : ainsi celle d'amandes amères, qui résulte du mélange de deux principes naturellement séparés dans la graine.

Les oléo-résines résultent du mélange d'une résine et oléo-résines. d'une essence. 0 n peut citer comme types: les térébenthines, qui découlent d'incisions pratiquées sur la tige de plusieurs espèces de Conifères, Mélèze, Pin, Sapin, etc., dans le bois et l'écorce desquels se trouvent accumulés les organes sécréteurs. La distillation de la térébenthine donne l'essence de ce nom et une résine, la colophane; citons encore l'oléo-résine désignée à tort sous le nom de baume de la Mecque ou de Judée, qui découle du Baumier de la Mecque, la térébenthine de Chio, celle de Copahu, improprement appelée baume de Copahu.

Les résines sont des corps solides à la température ordinaire, plus ou moins friables, à cassure vitreuse et d'odeur plus ou moins forte; nous nous contenterons d'en indiquer quelques-unes : la résine de gaïac, la résine mastic, fournie par le Pistachier lentisque, de la région méditerranéenne, et qui s'en écoule sous forme de larmes, à la suite de légères incisions du tronc et des branches vent comme masticatoire, pour parfumer l'haleine, fortifier les gencives et blanchir les dents, d'où son nom de mastic; la résine copals ou résine animée, produite par des plantes de la famille des Légumineuses, et qui sert à la fabrication de vernis siccatifs; la sandaraque, que fournit une espèce de Conifère du nord de l'Afrique, employée dans les vernis et pour rendre du corps au papier aminci par le grattoir; le jalap, utilisé en médecine comme purgatif.

Les gommes-r'ésines, résultant du mélange de résines et Gommes-résines. de matières gommeuses, sont produites par des plantes herbacées de l'Afrique et de l'Asie. Les principales sont l'asa fretilla, l'opoponax, la gomme ammoniaque, le galbanum, fournis par des plantes de la famille des Ombellifères, la myrrhe et l'oliban, par des Térébinthacées, la gomme-gutte, la scammonée, etc., par d'autres plantes.

Les baumes, enfin, sont des substances complexes, dans

Résines. 
lesquelles il entre des résines, des essences et un acide aromatique, tel que l'acide benzoïque ou cinnamique. Les plus employés sont le benjoin, le storax, le liquidambar, le baume de Tolu, le baume du Pérou, etc., qui sont utilisés en médecine et surtout dans la parfumerie.

Latex. - Les vaisseaux laticifères (fig. 17) renferment un suc spécial, le suc propre ou latex, formé de deux parties, un liquide incolore, dans lequel flottent en grand nombre des globules, tantôt incolores et tantôt vivement colorés en rouge, en jaune, en blanc laiteux. La consistance du latex est souvent plus ou moins visqueuse.

On n'est pas bien fixé sur le rôle que joue le latex dans l'économie de la plante. Autrefois on lui a attribué une importance considérable au point de vue de la nutrition. On croit généralement qu'il peut être repris par les cellules et concourir à l'alimentation ou à la calorification de la plante. Toutefois, dans un certain nombre de cas il paraît n'être plutôt qu'un produit excrémentitiel.

Il contient des substances très variables, qui diffèrent suivant les espèces végétales, et dont un grand nombre sont utilisées à des usages industriels ou médicaux, quelquefois même alimentaires; beaucoup renferment des poisons violents. Nous citerons quelques-uns des plus importants.

Le latex du Pavot somnifère blanc constitue l'opium; pour l'obtenil on incise superficiellement le fruit de la plante ou capsule; il s'en écoule un liquide laiteux qui se concrète en larmes que l'on recueille avec soin; celui de la Laitue, fourni par des incisions pratiquées au sommet de la tige, est connu sous le nom de lactucarium.

Le caoutchouc se rencontre dans le latex d'un assez grand nombre de plantes de l'Amérique du sud, de l'Inde et de l'Afrique; on l'obtient en faisant des incisions qui traversent toute l'épaisseur de l'écorce; la gutta-percha est le suc desséché d'un grand arbre de la Malaisie, l'Isonandra gutta; on l'obtient au moyen d'incisions faites sur le tronc.

Parmi les plantes qui fournissent un latex alimentaire, citons le Galactodendron utile ou l'arbre à la vache, de 
l'Amérique, dont le latex, semblable à du lait, en possède les propriétés nutritives; le Papaya carica, dont le latex contient une substance azotée, la papaïne, de même composition chimique que la pepsine retirée du suc gastrique des animaux, et qui, comme elle, a la propriété de digérer les substances albuminoïdes; aussi l'administre-t-on comme la pepsine aux personnes dont la digestion se fait mal.

Certaines plantes très utiles au point de vue alimentaire, telles que les Maniocs, qui donnent la cassave et le tapioca, sécrètent un suc propre très vénéneux, dont il faut avoir soin de débarrasser la fécule, sous peine d'empoisonnement; les Euphorbiacées en général, même celles de notre pays, renferment un suc très âcre et qui peut donner lieu à des accidents.

Le latex de l'Upas antiar et de plusieurs plantes voisines est très toxique et sert aux lndiens à empoisonner leurs flèches; celui qui s'écoule de plusieurs Champignons de notre pays est le plus souvent nuisible, mais il y a des exceptions, certains Champignons laiteux étant comestibles.

Enfin le latex peut contenir des résines, des baumes, du tannin, etc., ce qui ne doit pas surprendre, puisque, comme on l'a vu plus haut, il existe une série de formations intermédiaires entre les laticifères proprement dits et les appareils glandulaires chargés d'ordinaire de la sécrétion de ces substances. 


\title{
CHAPITRE III
}

\author{
ORGANOGRAPHIE VÉGÉTALE
}

OU

\section{ANATOMIE DESCRIPTIVE DES ORGANES DES PLANTES}

\section{Io ORGANES DE LA NUTRITION}

\section{Tige.}

Division des fonclions des végétaux. Division des organes de la nutrition. - Tige. Définition; notions générales sur la Tige. Tiges aériennes : ramification, forme, durée, consistance, port. Tiges souterraines : Rhizome, Bulbe, Tubercule. - Structure de la tige des Dicotylédones : système corlical; zone génératrice ou Cambium; système ligneux ou Bois. Développement de la Tige; le bourgeon terminal et l'accroissement de la Tige. Tableau résumé de la structure de la Tige. Modifications offertes par la tige de certaines Dicotylédones. - Structure de la tige des Monocotylédones. Modifications offertes par la tige de cerlaines Monocotylédones. - Structure de la tige des Acotylédones.

Division des fonctions des végÉtaux. - La Plante est un être vivant; elle doit par conséquent avoir, d'une part, la faculté de conserver, d'entretenir, d'accroître ses différentes parties, et d'autre part, celle de reproduire son espèce; d'où deux ordres de fonctions, celles de nutrition et celles de reproduction.

Toute l'activité de la plante tend vers l'un ou l'autre de ces deux buts; d'où une division toute naturelle des organes des végétaux, suivant qu'ils ont un rôle à jouer dans la nutrition ou dans la reproduction.

Nous commencerons cette étude par les premiers de ces organes et les fonctions qu'ils accomplissent; puis nous 
passerons au second groupe, à savoir les organes de la reproduction et leurs fonctions.

On le voit, il n'y a pas chez les végétaux, comme il en existe chez les animaux, un troisième ordre de fonctions, celles qu'on désigne sous le nom de fonctions de relation. Cependant, comme il se passe chez les premiers certains phénomènes qui ont quelque analogie avec des actes accomplis par les seconds se rapportant à la faculté de relation, un chapitre spécial leur sera consacré à la fin de l'ouvrage.

Division des organes de la nutrition. - Toutes les parties des végétaux qui concourenı à la nutrition, quelle que soit d'ailleurs leur forme, leur dimension et leur structure, peuvent se ramener à l'un ou l'autre de ces trois organes: la tige, la racine, les feuilles.

Il en est du moins ainsi chez les Phanérogames, car chez les végétaux inférieurs de l'embranchement des Cryptogames, les parties sont moins compliquées, de sorte qu'il n'y a plus lieu de décrire des organes aussi nettement différenciés.

Définition DE LA TIGE. - La tige est cette partie de la plante, qui, en général, s'élève en l'air et se dirige vers la lumière, par conséquent dans une direction opposée à celle de la racine; mais parfois elle rampe sous le sol comme une vraie racine. Le caractère essentiel de la tige, celui qui la distingue constamment de la racine, c'est qu'elle porte des feuilles, tandis que celle-ci n'en offre jamais.

Notions générales sur li tige. - Il est utile, avant d'aborder la description anatomique de la tige, de donner certaines indications relatives aux principales particularités qu'offre cette partie des végétaux, au point de vue de la forme, de la consistance, de la durée, de la direction, etc. Nous les étudierons d'abord dans les tiges aériennes, puis dans les tiges souterraines. 
10 Tiges aériennes.

A. Existence apparente ou cachée. La tige existe constamment, mais elle est parfois très réduite, ou bien cachée sous le sol, de sorle que l'on pourrait croire à son absence; on dit alors que la plante est acaule (a privatif, caulis, tige), par

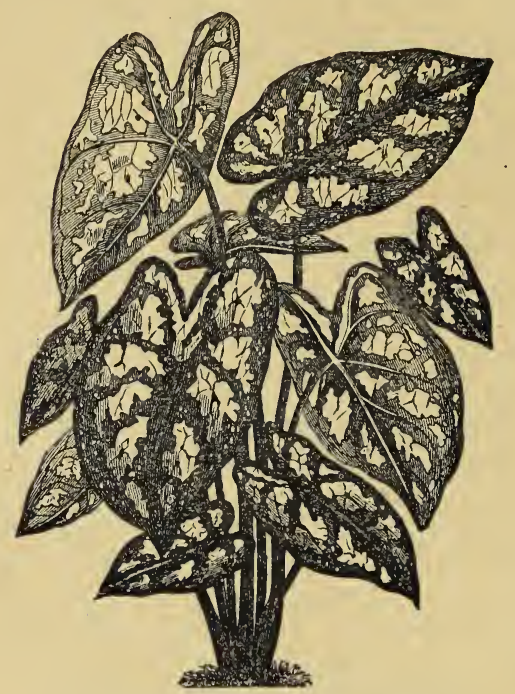

Fig. 33. - Plante acaule (Caladiumi.

opposition à celles qui ont une tige apparente, bien développée, et sont dites caulescentes.

B. Ramification. La tige est tantôt simple, c'est-à-dire réduite à l'axe principal ou primaire, tantôt plus ou moins ramifié, et pourvue alors de ramifications ou axes secondaires, tertiaires, etc., vulgairement appelés branches, rameaux, ramuscules. Ces axes successifs peuvent se former par bifurcations (fig. 34) ou trifurcations de l'axe précédent, et la tige est dite alors dichotome ou trichotome. Si les rameaux naissent de la base même de la tige de façon à se trouver en contact avec le sol et à y enfoncer même des racines, ils portent le nom de drageons. Sur les arbres de nos forêts les rameaux ne partent que d'une certaine hauteur, et leur ensemble constitue ce qu'on appelle la cime. Si les rameaux sont dressés, presque verticaux (Peuplier d'Italie), la cime est dite fastigiée; la disposition inverse, bien connue dans certains Saules ou Sophoras, mérite à ces arbres le nom de pleureurs; enfin dans la position intermédiaire, les rameaux sont horizontaux et dits étalés (Cèdre du Liban). 
C. Forme. Le plus souvent la tige est arrondie, cylindrique; parfois elle est aplatie ou comprimée, triangulaire, comme dans les Carex, ou bien quadrangulaire (Labiées).

D. Durée. La durée de la tige n'est pas toujours égale à celle de la plante elle-même. Lorsque celle-ci est annuelle, c'est-à-dire subit en un an toute son évolution et périt ensuite, la tige est également annuelle

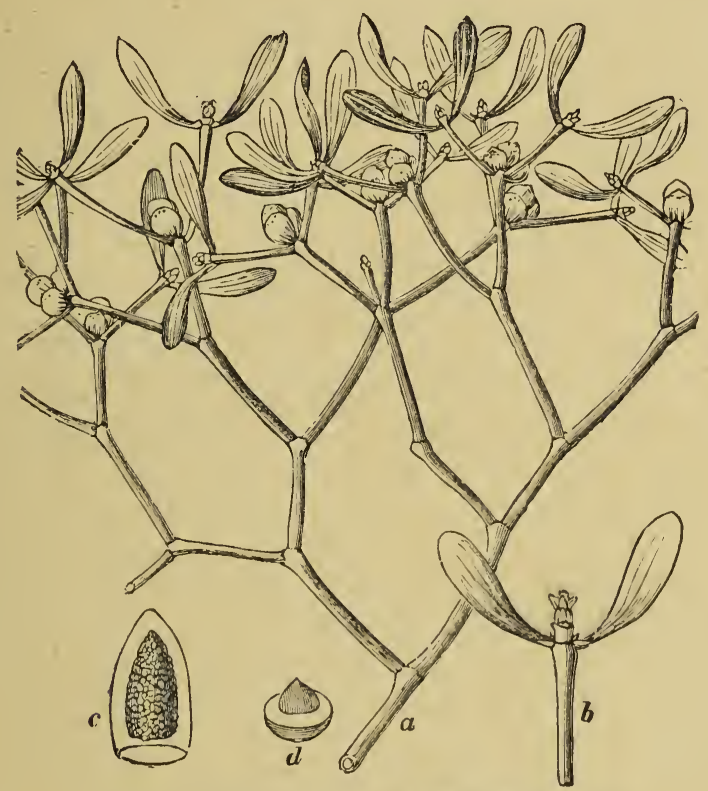

Fig. 34. - Tige à ramification dichotomique (Gui).

(Chanvre, Lin, Blé).ll en est de mème pourles plantes bisannuelles, c'est-à-dire qui mettent deux ans à germer, croître et fructifier, pour mourir après (Carotte, Betterave). Mais parmi les plantes vivaces, c'est-à-dire qui vivent un grand nombre d'années, les unes conservent leurs tiges et leurs rameaux dans leur totalité ou en grande partie, ce qui est le cas de tous les végétaux ligneux, tandis que les autres perdent chaque année toutes leurs parties extérieures après la fructification, et la plante ne continue à exister 
que par ses parties souterraines, à savoir, les racines et la base de la tige, ou par un rhizome (plantes herbacées vivaces : Nénuphar, Guimauve, etc.)

$\mathrm{Au}$ lieu de classer les plantes d'après leur durée absolue, on se base souvent sur la propriété qu'elles ont de produire leurs fruits une seule fois, pour mourir peu après, ou au contraire, plusieurs années de suite : ainsi on appelle monocarpiennes ou monocarpiques celles qui après avoir produit leurs fruits meurent aussitôt, résultat qui, pour s'accomplir demande un temps très variable suivant les espèces. Ainsi, pendant que le Blé se développe et mûrit ses graines dans une période d'une année, l'Agave americana y emploie 10 à $1 \zeta$ ans dans son pays d'origine, et 50 à 60 ans dans notre climat. De même, on appelle polycarpiennes ou polycarpiques, célles qui produisent des fruits pendant plusieurs années.

$\boldsymbol{E}$. Consistance. La tige est dite ligneuse quand elle est incrustée d'une matière dure qui forme le bois, comme

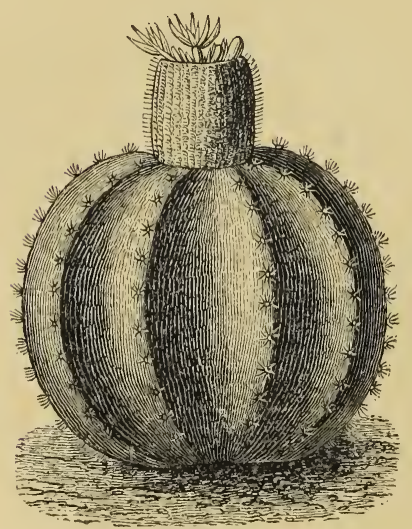

Fig. 35. - Plante grasse (Echinocactus). sont les arbres de nos forêts ; herbacée, lorsque sa consistance est molle, comme celle des herbes; frutescente, celle dont la consistance est intermédiaire aux deux précédentes, les extrémités des rameaux seuls ne se lignifiant pas et se détruisant chaque année; charnue, enfin, celle qui, tout en étant vivace, reste très molle, gorgée de sucs, ce qui a valu aux végétaux qui offrent ce

caractère le nom de plantes grasses.

F. Port. Citons d'abord trois sortes principales des tiges considérées surtout au point de vue de leur port, de leur physionomie en quelque sorte : le tronc, auquel 
appartient la lige des arbres de nos forêts, dont la base est sensiblement plus large que le sommet et qui porte

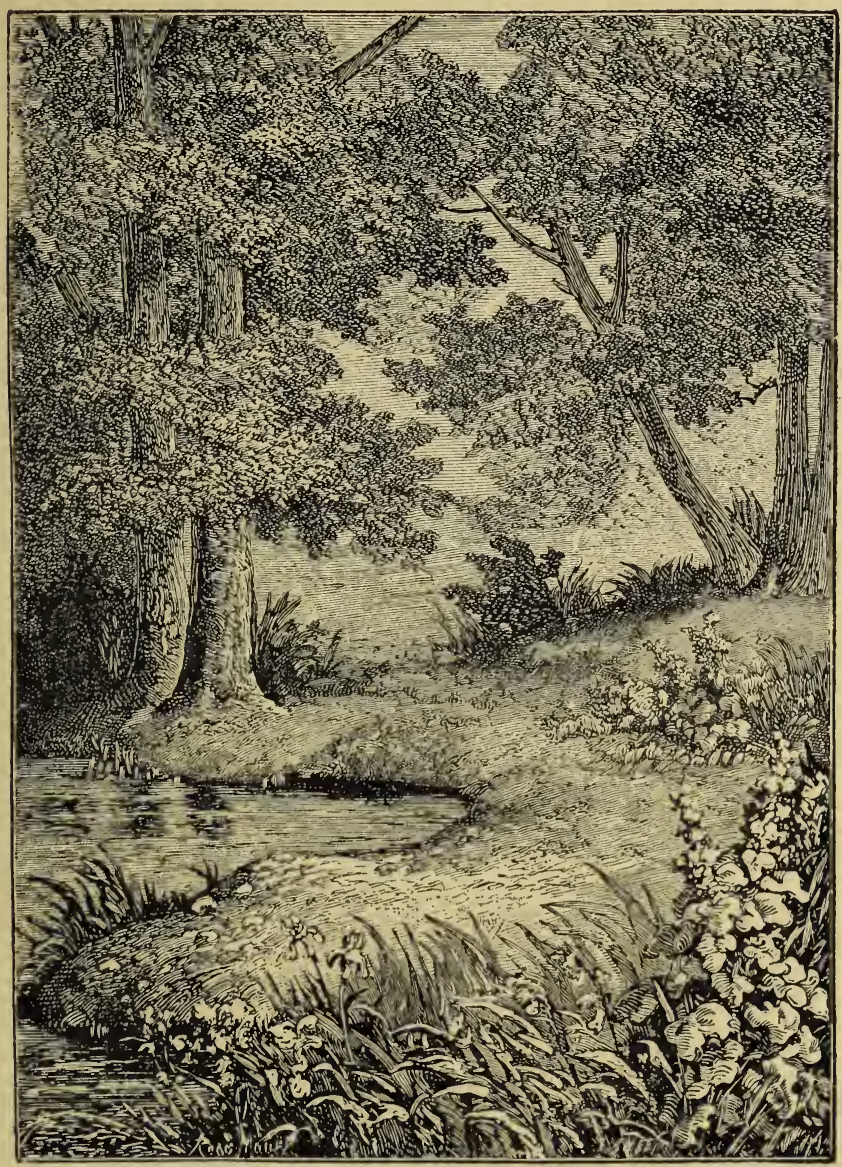

Fig. 36. - Tronc des arbres de nos forêts.

un grand nombre de rameaux; le stipe, tige d'un grand nombre de Monocotylédones arborescentes (Palmiers, Dracœna), qui s’élevant comme une colonne droite, à peu 
près de même diamètre à la base et au sommet, n'offre pas de ramifications, mais se termine par un énorme

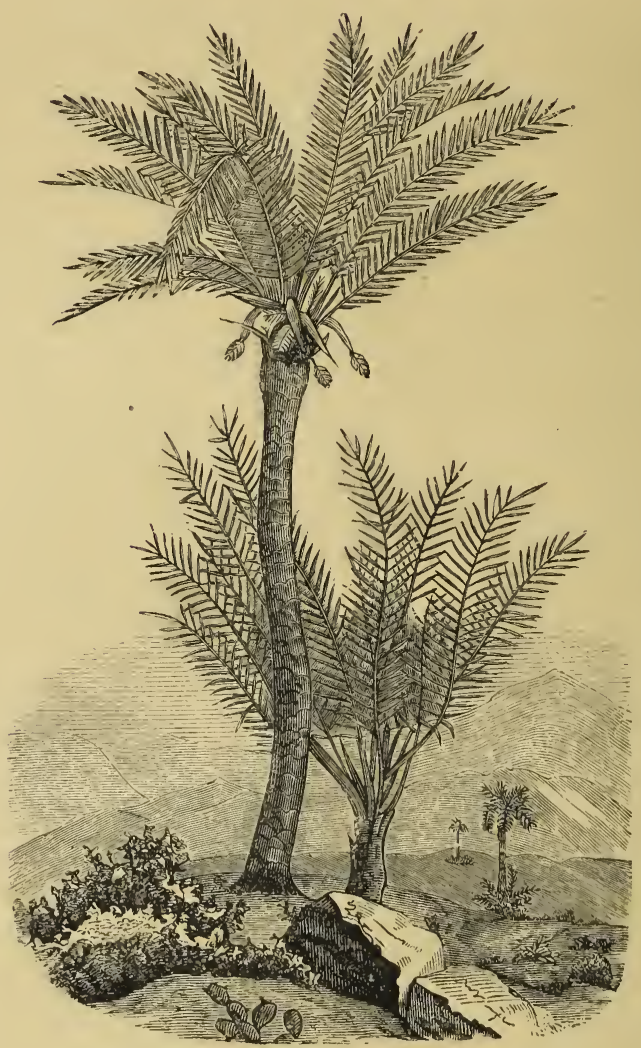

Fı. 37. - Stipe des Palmiers.

bouquet de feulles; le chaume, qui peut être de consistance herbacée (Blé, Seigle, etc.) ou ligneuse (Bambous), est creux à l'intérieur et coupé d'entre-nœuds qui divisent sa cavité par autant de cloisons horizontales d'où partent les feuilles.

Mais il existe bien d'autres traits caractéristiques de la tige 
au point de vue du port ou de la direction : quelques-unes sont obliqnies, c'est-ì-dire plus ou moins inclinées; d'autres

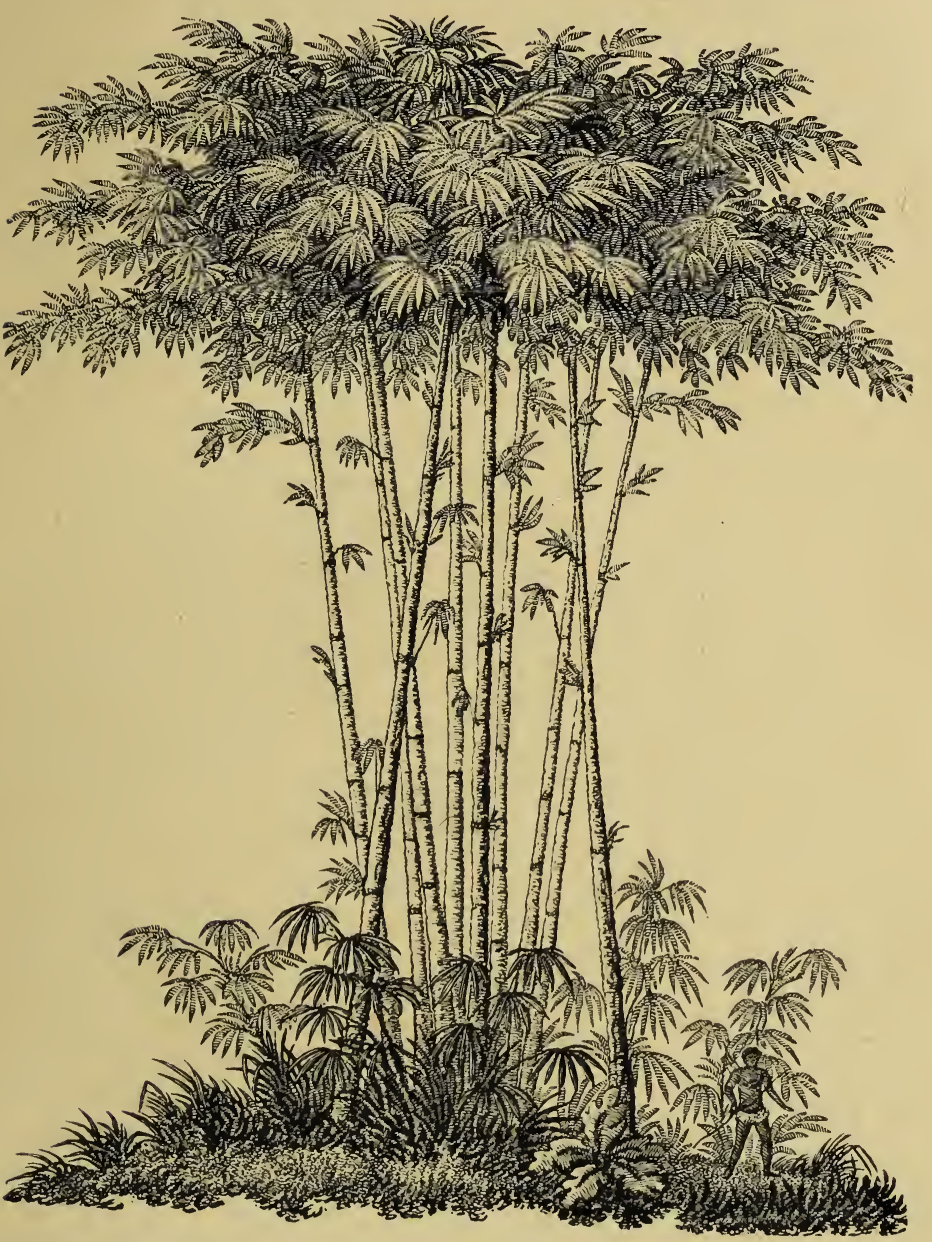

Fig. 38. - Chaume ou tige fistulense des Bambous.

couchées et reposent par toute leur longueur sur le sol, en s'y fixant par de petites racines adventives qui naissent à son contact, et qui donnent de distance en 
distance des rameaux grêles, désignés sous le nom de cou_ lants, gourmiands ou stolons (Fraisier).

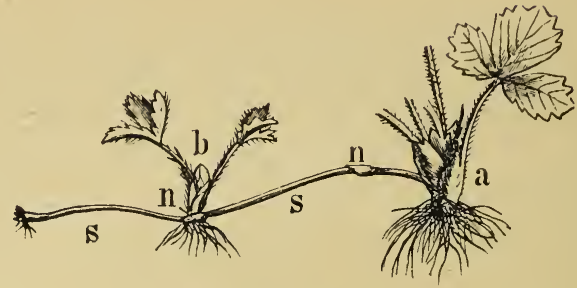

Fig. 39. - Fraisier (a) d'où est sorti un stolon (ns), lequel au contact du sol a produit des racines et une tige nouvelle ( $b$, d'où sort un nouveau stolon (ns).

Certaines tiges, tout en étant ligneuses, sont cependant trop faibles pour se tenir verticales et sont obligées de s'appuyer

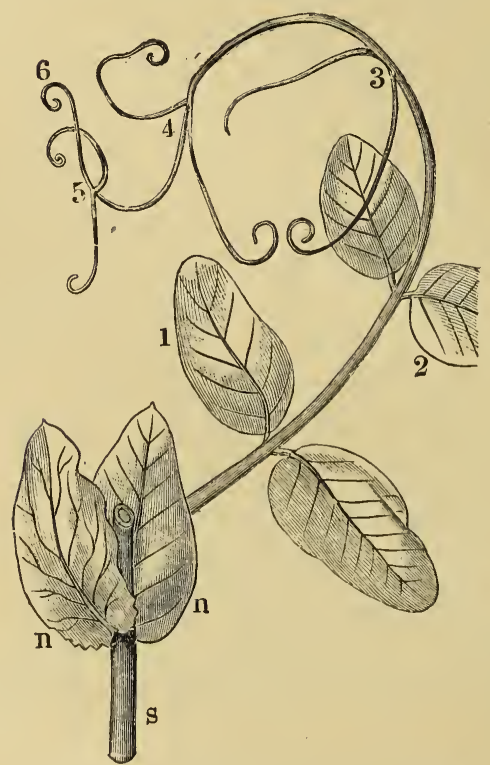

Fig. 40. - Rameau de Haricot (Pisum sutivum) dont les feuilles opposées deux par deux sont remplacées à l'extrémité de la tige par des vrilles ou cirres.

la plante reprend d'elle-même surle sol ou de s'accrocher aux corps voisins au moyen de certains appendices appelés vrilles (Vigne, Chèvrefeuille), on les appelle sarmenteuses (fig. 41); d'autres s'attachent, s'incrustent au moyen de crampons sur les arbres ou les murs voisins et sont dites grimpantes (Lierre); d'autres encore, appelées volubiles, s'enroulent autour d'un support quelconque el la plupart toujours dans le même sens, pour une espèce donnée, de sorte que si l'on vient à contrarier cette direction normale sa position première, 
ou finit par périr; celles qui s'enroulent de gauche à droite (Haricot) sont dites dextrorsum, et celles qui le font de droite à gauche, sinistrorsum, (Houblon, Chèvre-feuille).

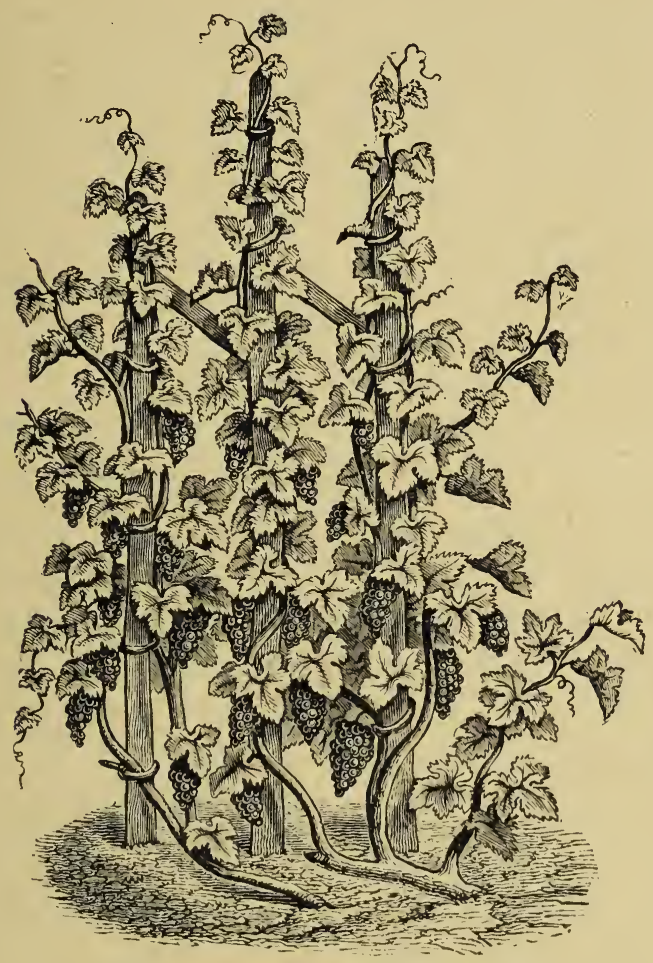

Fig. 41. - Tige sarmenteuse (Vigne).

Parfois la plante grimpante enserre si étroitement l'arbre qui lui sert de tuteur, qu'elle s'y enfonce profondément à travers l'écorce, en arrêtant la sève de retour, et finit par étouffer son support, comme le Chèvre-feuille en offre de fréquents exemples dans nos bois. Le type des plantes grimpantes, le plus célèbre par son incroyable développement, est celui des lianes des pays chauds, qui, tout en conservant constamment un faible diamètre, atteignent une 
longueur qui peut aller jusqu'à 300 mètres, et qui s’élançant debranche en branche et d'arbre en arbre, contribuent à transformer parfois les forêts intertropicales en fourrés inextricables.

G.Désignation des plantes phanérogames, d'après leur port, leur dimension el leur consistance. Les herbes sont des tiges molles, complètement herbacées; les sous-arbrisseaux, des tiges ligneuses avec les rameaux herbacés (Sauge, Vigne vierge, Clématite); les arbustes, des tiges ligneuses, ramifiées dès la base et ne dépassant pas la taille d'un homme (Groseiller); les arbrisseaux, des tiges ligneuses ramifiées dès leur base et pouvant avoir trois ou quatre fois la taille d'un homme; les arbres, enfin, ont un tronc ligneux s'élevant à cinq ou six mètres au moins et ne poussant d'ordinaire des branches qu'à partir d'une certaine hauteur, pour constituer une cime.

II Tiges souterraines.

Tandis que toutes les sortes de tiges qui viennent d'être passées en revue méritent le nom d'aériennes, puisqu'elles s'élèvent au-dessus du sol, celles d'un nombre considérable de plantes méritent en raison de leur situation celui de souterraines. On en distingue trois sortes: les rhizomes, les bulbes et les tubercules.

A. Rhizomes. On peut les considérer comme des tiges rampantes qui, au lieu de s'étaler à la surface du sol, rampent au-dessous de lui. Les uns sont définis, les autres indéfnis : dans le premier cas, l'axe principal sort de terre par son extrémité et produit une ou plusieurs fleurs, qui meltent un terme à sa végétation; mais à l'aisselle d'une des feuilles portées par cette tige souterraine naît un axe secondaire qui, après s'être développé horizontalement dans le sol, se relève à son tour comme l'axe primaire, puis meurt après avoir produit une fleur, et ainsi 
de suite d'année en année. Dans le deuxième cas, l'axe principal reste loujours caché dans le sol ets'y accroîtindéfiniment

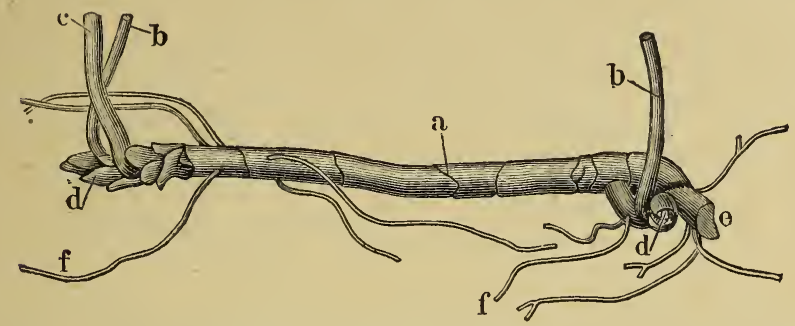

Fig. 42. - Rhizome ou tige souterraine d'Anémone (grand.nat.); $a$, cicatrices de feuilles; $b$, pédoncules foliaires; $c$, hampe florale; $d$, bourgeon foliaire; $e$, section du rhizome; $f$, racines.

par son extrémité; nuais de distance en distance, il donne naissance à un rameau qui porte des feuilles et des fleurs et

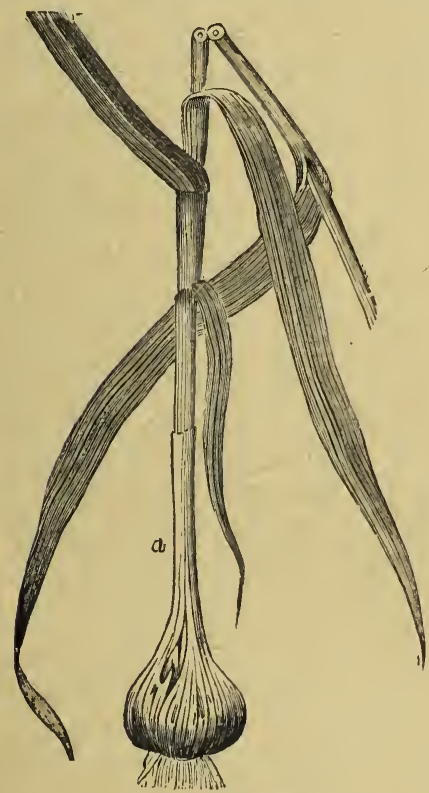

Fig. 43. - Bulbe tuniqué. qui meurt chaque année.

Dans les rhizomes, à mesure que la plante vieillit, la portion ancienne se détruit, tandis que la partie plus récente tend à s'éloigner de plus en plus du point de départ, de sorte que chaque année les rameaux aériens apparaissent à une place un peu différente de celle de l'année précédente. Comme exemple de rhizomes on peut citer l'Iris, le Sceau de Salomon.

B. Bulbe. Les bulbes sont une modification du rhizome; au lieu de s'allonger, ils restent très raccour'cis et sont formés d'une sorte de platealu arrondi, de la face inféricure duquel par- 
tent des racines, tandis que la face supérieure porte un bourgeon enveloppé d'écailles, qui se transformera en feuilles et
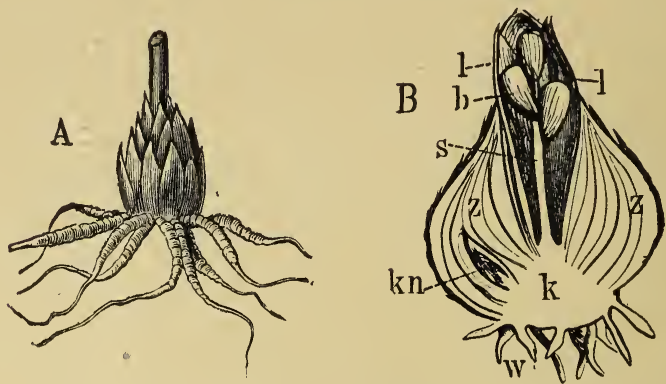

Fig. 44. - Bulbes écailleux; A, du Lis martagon, avec ses racines; B, de la Jacinthe, coupé en long; $k$. plateau; $k n$, un bourgeon ou caïcu; $z$, écailles du bulbe; $l$, enveloppe de l'inflorescence; $b$, fleurs non épanouies ; $s$, hampe florale; $w$, racines.

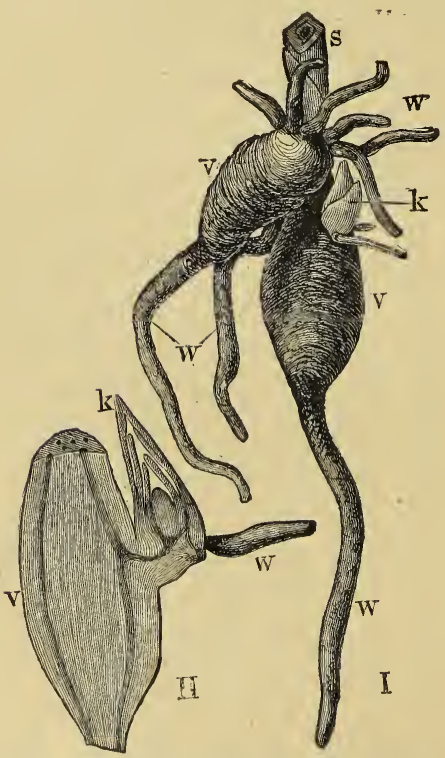

Fig. 45. - Bulbe plein de la Jacinthe sylvestre (Platanthera bifotia); I, vue d'ensemble, $v, v$, bulbes ou tiges souterraines renflées; celui de droite est en pleine activité; $k$, bourgeon foliaire; $w$, racines; $s$, hampe florale coupée; II, bulbe de la même plante coupé en long; $k$, bourgeon; $w$, racines. en fleurs. Sur les côtés du plateau, à l'aisselle des feuilles, naissent des bourgeons secondaires, appelés caïeux.

On distingue plusieurs variétés de bulbes: lesbulbestuniqués, dont les écailles se recouvrent complètement les unes les autres, comme autant de tuniques; on les appelle vulgairement oignons (Poireaux, Oignons comestibles, Jacinthes) ; les bulbes écailleux, dont les écailles charnues sont imbriquées à la façon des tuiles d'un toit (Lis blanc); les bulbes solides, dont 
toute la masse est solide et pleine, par suite de l'épaississement de l'axe, que recouvrent seulement quelques minces tuniques sèches (Glaïeul, Jacinthe sylvestre).

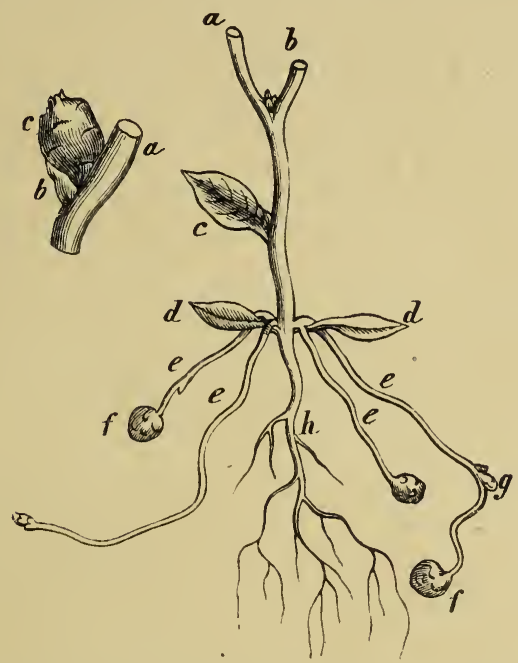

Fig. 46. - Tige et racine de la Pomme de terre; $a, b$, rameaux aériens coupés : $c, d$, feuilles; $e, e$, rameaux souterrains renflés à leur extrémité en tubercules $f / ; g$, bourgeon souterrain $h$, racine. A gauche et en haut un bourgeon souterrain isolć.

C. Tubercules. Ce sont encore des tiges souterraines, formées d'une masse renflée, épaisse, charnue et ordinairement gorgée de fécule (Pomme de terre, Topinambour). Les petits enfoncements que présente la surface des tubercules de pomme de terre et que les jardiniers appellent les yeux, sont occupés par de petits bourgeons dont chacun suffit à reproduire la plante entière.

\section{STRUCTURE DE LA TIGE}

A. Dicotylédones.

Parties constitutieg de la tige. - Pour se rendre compte de la structure de cette partie des végétaux, il faut prendre 
une tige d'un an ou deux, et avec un rasoir bien affilé en détacher une rondelle aussi mince que possible, d'autre part, fendre le rameau dans sa longueur et en couper de même une mince lamelle parallèlement à son axe. Ces deux préparations examinées au microscope permettront de se rendre compte à la fois de la forme des éléments constituants et de la façon dont il sont agencés.

C'est ainsi que l'on constatera que la tige se compose, en allant de l'extérieur vers l'intérieur, de trois systèmes distincts concentriques l'un à l'autre, à savoir : un extérieur, le système cortical, comprenant l'épiderme, le liège ou couche subéreuse, le parenchyme cortical ou couche herbacée et le liber; un intermédiaire, la zone génératrice ou cambium; un interne, le système ligneux, comprenant le bois et la moelle.

\section{Système cortical.}

$1^{\circ}$ Epiderme. - L'épiderme forme une couche continue parfaitement distincte, souvent hérissée de poils formés aux dépens de ses cellules, entre lesquelles se voient les orifices microscopiqués d'un assez grand nonıbre de stomates, petits organes qui seront étudiés à propos de la structure des feuilles. L'épiderme peut être formé d'une couche unique de cellules, ou bien de 2, 3 ou 4 couches superposées. Le caractère dominant de 'ces éléments est d'être aplatis, transparents, incolores; ils sont fortement reliés entre eux, de sorte que l'épiderme peut facilement s'arracher par lambeaux (fig. 47 et 48 ).

Cuticule. La surface extérieure de l'épiderme est r'ecouverte d'une sorte de vernis, sous forme d'une pellicule très mince, qu'on appelle la cuticule, et dont le rôle est de protéger les cellules épidermiques contre l'action direcle des agents extérieurs. Bien qu'elle soit une production de l'épiderme, 
la cuticule a une composition chimique différente, car elle n’offre pas comme lui les réactions propres à la cellulose.

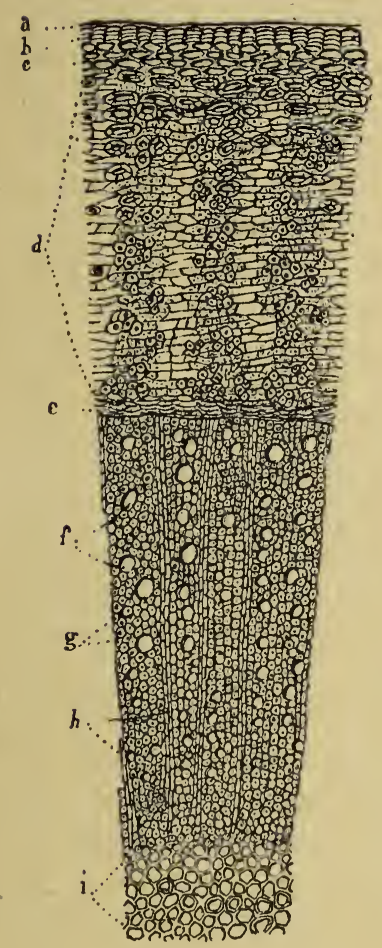

Fig. 47. - Segment d'une coupe transversale d'une jeune tige (Camphrier de Bornéo); $a$, cuticule; $b$, épiderme et couche subéreuse; $c$, parenchyme herbacé ; $d$, liber; $e$, cambium ; $f$, vaisscaux du bois; $g$, fibres ligneuses; $h$, rayons médullaires; $i$, moelle. $2^{\circ}$ LiÈge ou Couche SUBÉreuse. - Cette portion de l'écorce est formée par des éléments que l'on doit regarder comme privés de vie. Ce sont des cellules aplaties, de couleur foncée, serrées les unes contre les autres et alignées avec une assez grande régularité, ne contenant plus trace de protoplasma, en même temps que leur réaction chimique, en présence de la teinture d'iode et de l'acide sulfurique, n'y décèle plus l'existence de la cellulose. Ces cellules ont été vivariles à un moment donné et faisaient partie du parenchyme épidermique ou du parenchyme cortical, puis elles se sont subérifiées (fig. $47, b ; 48, c$ ). Dans certaines tiges le suber prend un développement énorme, se forme avec une grande régularité et présente des propriétés qui permettent de l'employer à divers bien connu de liège. Le ou Chêne-liège, que l'on 
cultive en Algérie, précisément pour mettre son écorce en coupe réglée (1).

Périderme. Chaque zone annuelle de liège est séparée de celle qui se produit l'année suivante par une couche de cellules d'aspect différent, à parois bien

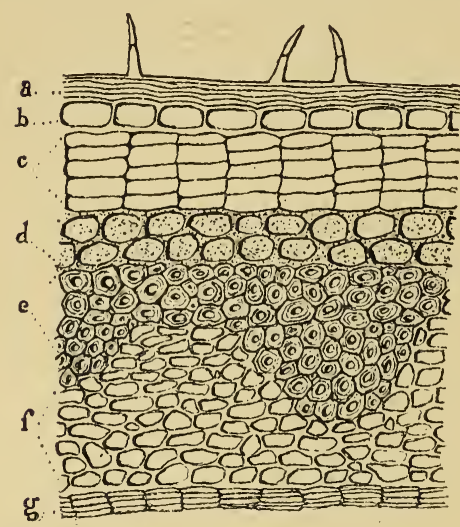

Fig. 48. - Coupe transversale de l'écorce très grossie; $a$, cuticule sur laquelle se voient plusieurs poils; $b$, épiderme; $c$, liège ou couche subéreuse; $d$, parenchyme herbacé ; $e$, fibres libériennes; $f$, cellules du parenchỵme libérien; $g$, zone génératrice ou cambium. plus épaisses, à laquelle on donne le nom de périderme, et qui peut même avoir une épaisseur plus grande que le liège lui-même. Il en est ainsi dans le Bouleau, le Platane, où le périderme forme des feuillets superposés résistants et à peu près incorruptibles à l'eau. LesGanadiens font avec cette partie de l'écorce prise sur le Bouleau papyracé, des canots d'une extrême légèreté et assez résistants.

Mais il peut se faire que des lames de ce genre se produisent jusque dans l'épaisseur du liber et isolent complètement certaines portions de celui-ci qui, dès lors, perdent forcément leur activité nutritive, meurent et tombent par grands lam-

(1) Quand le Chêne-liège atteint l'âge de dix à quinze ans, on trace sur son écorce des incisions longitudinales, puis on enlève les lames d'écorce ainsi circonscrites, c'est le démasclage; le liège ainsi obtenu manque de souplesse, est tout crevassé, sc prête mal aux usages industricls, les ouvriers l'appellent liège mále; son enlèvement met à nu l'enveloppe herbacée; e'est ectte partie jointe au liber que les ouvriers appellent le lard ou la mère, parecque e'est elle qui formela du liège nouveau. $\Lambda u$ bout de sept à huit ans, un liège beaucoup plus fin et plus souple s'est développé; c'est le liège femelle, qui est enlevé à son tour. Un Chêne-liège peut durer environ cent cinquante ans $\mathrm{et}$ fournir 18 à 20 récoltes. 
beaux, comme cela se voit chaque année sur nos Platanes, Bouleaux, Cerisiers, etc.

C'est une formation analogue qui détermine ces crevasses rugueuses dont l'écol'ce d'un grand nombre de nos arbres est sillonnée; mais dans ce cas, au lieu de se détacher complètement, les parties enveloppées de périderme continuent à adhérer au reste de l'écorce; c'est à cette écorce ainsi crevassée que l'on a donné le nom de rhytidome (putis, ride, et $\delta \tilde{\omega} \mu \alpha$, couverture); tout le monde a pu observer cette particularité sur le Pin, le Chêne, l'Orme el bien d'autres arbres.

D'autres formations apparlenant au suber sont appelées Lenticelles. lenticelles, nom qui rappelle la forme de lentilles qu'elles présentent. Ce sont de petites proéminences du liège à travers les parties superficielles de l'écorce, probablement au niveau des petites fentes des stomates. Elles se voient nettement sur les rameaux de l'année chez les Dicotylédones ligneuses, mais manquent dans les autres embranchements; elles sont des plus manifestes sur le Bouleau et le Fusain.

Remarque. - Pour être complet dans l'énumération des zones constitutives de l'écorce, disons qu'entre le liège et le parenchyme cortical, qui va être étudié, on trouve souvent une couche de cellules variables dans leur forme, à parois souvent très épaisses, et que l'on désigne sous le nom de sous-épiderme, mésoderme, hypoderme.

3" Parencinie contical. - Appelée encore couche herbacée, celte partie de l'écorce renferme une grande quantité de matière verte ou chlorophylle qui, sur les jeunes rameaux, se voit à travers la couche subéreuse et l'épiderme transparents (fig. 48, d). Elle atteint son maximum de développernent dans les plantes herbacées. Elle est formée de cellules globuleuses, polyédriques, à parois minces et en pleine voie d'activité; aussi est-elle le siège de l'élaboration d'un certain nombre de produits, tels que fécule, sucre, gomme, latex, etc. 
$4^{\circ}$ Liber. - Celte zone qui doit son nom à ce qu'elle est formée de couches minces superposées, qui peuvent même se lever comme les feuillets d'un livre, se compose de plusieurs sortes d'éléments. On y trouve, en effet, un tissu cellulaire, qui est très

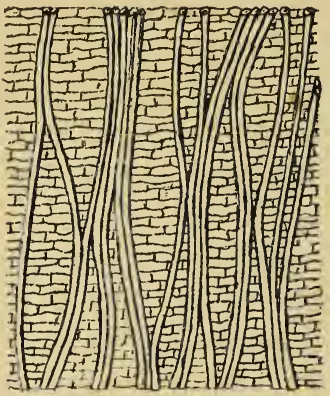

Fig. 49. - Coupe longitudinale d'un fragment d'écorce montrant la marche des faisceaux de fibres du liber, entre lesquels se voit le tissu cellulaire ou parenchyme libérien. analogue à celui de la couche herbacée, et au milieu duquel se trouvent plongés des faisceaux de fibres, síparés les uns des autres par ce tissu (fig. 49). Ces faisceaux de fibres libériennes ont une direction générale verticale, mais en même temps plus ou moins flexueuse, jusqu’à venir même au contact les uns les autres et se souder fortement dans ces points de jonction, de manière à former une sorte de treillis qui offre en général une grande résistance. Cette disposition est aussi manifeste que possible dans un arbre des Antilles, le Laghetta lintearia, chez lequel les feuillets du liber ressemblent à une dentelle, d'où le nom de Boisdentelle donné à cette plante.

Les fibres du liber sont des tubes à parois épaisses mais non lignifiées et restées flexibles, dont le canal très étroit peut même diminuer jusqu'à s'oblitérer complètement, dans le Chanvre, par exemple, et qui se terminent en pointe oblique à leurs deux extrémités fortement soudées à celles des fibres voisines (fig. 14). Ces fibres contribuent à donner de la solidité à la plante et livrent passage aux fluides gazeux qui circulent dans son intérieur.

Ce sont ces mêmes fibres qui constituent la partie textile du Chanvre, du Lin, de l'Ortie, du China-grass (Urtica nivea) très employé en Chine, et de quelques autres plantes recherchées pour la fabrication des tissus. Par le rouissage, c'est-à-dire la macération dans l'eau, 
ces fibres se séparent les unes des autres par suite de la destruction de leur gangue, formée des cellules du parenchyme libérien au milieu desquelles elles se trouvent plongées.

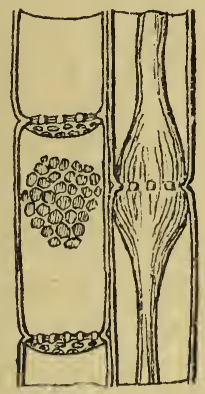

Fig. 50. - Cellules grillagées. A gauche, on voit une cellule coupée en long, dont chaque extrémité est fermée par une sorte de crible horizontal; sur un point de la paroi se voit une disposition semblable. A droite, deux autres cellules, séparces par un diaphragme criblé qui permet au protoplasma de passer de l'une à l'autre.

Dans une très jeune branche, les faisceaux libériens sont disposés sur une seule ligne circulaire (fig. 48,e); mais par les progrès de la végétation, de nouveaux faisceaux apparaissent en dedans des premiers, et dans lecours de l'année il peut se former ainsi jusqu'à cinq rangées de faisceaux concentriques (fig. $47, d$ ).

D'autres éléments impor- Tubes cribreux tants du liber consistent grillagées. dans ce qu'on appelle les tubes cribreux el les cellules grillagées. Ce sont des tubes ou des cellules larges, dont les parois minces présentent d'énormes ponctuations sur lesquelles s'étend une membrane excessivement délicate marquée d'un fin réseau qui lui donne l'aspect d'un crible microscopique (fig. 50). Elles sont extrêmement communes dans nombre de végétaıx, entre autres le Poirier, le Tilleul, le Noyer, etc.

\section{Zone génératrice ou Cambium.}

Constitution et Rôle nu Gavbium. - Interposée entre les tissus précédents, réunis vulgairement sous le nom d'écorce, et ceux qui vont être bientôt étudiés sous le nom de bois, 
cette zone est formée de cellules à parois minces, très actives, gorgées de sucs, et qui ont la propriété de se multiplier activement surtout au printemps, pour former du côté extérieur de nouvelles couches de liber, et du côté interne, de nouvelles couches de bois. Chaque année une zone nouvelle se forme de la sorte de part et d'autre du cambium, de façon à donner à la tige un diamètre de plus en plus considérable (fig. $47, e ; 48, g$ ).

\section{Système ligneux ou Bois.}

$1^{\circ}$ Bois prophement itt. - La coupe transversale d'un tronc d'arbre ou d'une branche de plusieurs années nous montre des couches circu-

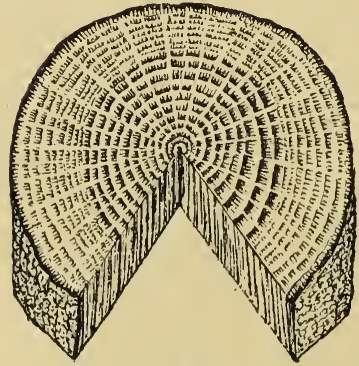

Fig. 51. - Tronçon de Chêne montrant le cœur du bois, plus foncé, l'aubier en dehors, les rayons mélullaires, allant de la périphérie au centre, et enfiñ l'écorce. laires emboîtées les unes dans les autres autour d'une partie centrale nomméc le canal médullaire. De plus, on voit que de celui-ci partent des lignes étroites qui vont en rayonnant vers la périphérie du bois, lequel se trouve par là même divisé en autant de triangles, dont la base est en dehors, la pointe en dedans. Quelle est la signification de ces particularités?

Les zones concentriques représentent chacune une année de végétation et ont toutes la même structure; il suffit donc d'en étudier une pour connaître toutes les autres (1).

(1) La disposition en zones concentriques distinctes n'est guire accusée que dans les arbres des pays froids ou tempérés, c'est-àdire ceux où la végétation offre, chaque année, une période de grande 
Quant aux lignes rayonnantes qui s'étendent de la moelle à l'écorce, ce sont les r'ayons médullaires, restes du tissu dans lequel se sont développés les faisceaux vasculaires qui constituent la plus grande partie du bois.

Ajoutons qu'assez souvent la masse du bois est en outre nettement divisée en deux régions distinctes, les zones inté-

Coeur du bois et Aubier. rieures étant d'une couleur plus sombre, d'un tissu plus serré, plıs dur; celles de l'extérieur ayant une nuance plus claire, un tissu bien moins résistant. Les premières, qui sont formées depuis plus longtemps, constituent le duramen ou cœur du bois, tandis que les autres, de formation plus récente, constituent l'aubier. Le Chêne présente d'une façon très nette cette distinction en aubier et en duramen; dans la bonne menuiserie on n'emploie que cette dernière partie. Lorsque l'arbre est jeune, la quantité d'aubier est en beaucoup plus grande proportion que plus tard; aussi convient-il de n'abattre les chênes que lorsqu'ils ont au moins 100 ou 150 ans.

Le bois se compose de fibres et de vaisseaux. Les premières offrent les caractères que nous savons appartenir à celles du liber, c'est-à-dire, qu'elles ont la forme de tubes Vaisseaux du bois. étroits terminés en pointe, soudés les uns aux autres et anastomosés pour former des réseaux (fig. 14 et 49). Elles peuvent présenter les ponctuations ou les épaississements variés décrits plus haut; enfin elles s'incrustent de ligneux et deviennent très résistantes.

Les seconds, épars au milieu des fibres, sont ponctués ou rayés ou quelquefois annelés. Avec le temps, leur's parois d'abord minces s'épaississent et leur calibre diminue par suite du dépôt du ligneux qui leur donne une grande consistance, en même temps qu'une couleur plus foncée.

2o Etur médullaire. - C'est la partie la plus interne du bois, celle qui entoure immédiatement la moelle. Des carac-

activité, laquelle va en se ra lentissant, pour être bientôt suivie d'une période de repos. Cependant, même dans ces pays, toutes les plantes n'en sont pas pourvues. Dans les pays chauds, la végétation ne subis sant pas d'arrêt, le bois ne forme qu'une masse homogène. 
tères très nets la distinguent du reste du système ligneux. Elle offre, en effet, des éléments spéciaux, à savoir, des vaisseaux annelés, que nous avons vus exister rarement dans le reste du bois, mais surtout des vaisseaux spiralés ou trachées à spire déroulable, que l'on rencontre uniquement dans cette région de la tige.

$3^{\circ}$ Rayons médullaires. - Comme il a été déjà dit, ce sont les restes du tissu cellulaire ou parenchyme, dans lequel se sont développés les éléments du bois, qui constituent les rayons médullaires. Refoulé de plus en plus, le tissu cellulaire se trouve réduit à l'état de minces cloisons interposées entre ces éléments. Les unes sont plus longues et traverscnt toute l'épaisseur du bois; les autres, plus courtes, ne comprennent qu'un nombre variable de zones ligneuses, mais partent toujours des plus extérieures pour s'avancer plus ou moins loin vers le centre.

Les cellules constituantes des rayons médullaires sont allongées de dedans en dehors (fig. 47, $h$ ), contrairement aux cellules des autres parties de la tige qui sont allongées dans le sens transversal; leur forme est généralement quadrilatère.

$4^{0}$ Moelle. - Partie la plus interne de la tige, la moelle est formée de cellules ordinairement hexagonales, remplies de suc et souvent colorées en vert dans les jeunes rameaux ; mais bientôt, par suite des progrès de la végétation, tous les sucs dont elle est gorgée sontemployés au développement des feuilles et des fleurs, et dès lors ses cellules constituantes sont desséchées, vides et décolorées. Souvent même, ces cellules se rompent, de larges cavités sont creusées au milieu d'elles, et les débris de la moelle ne se voient plus que sur le pourtour de l'étui médullaire.

La moelle renferme à l'état jeune, comme il a été dit, des substances diverses, dont plusieurs sont extraites par l'industrie, qui les fait servir à notre alimentation, ou ì d'autres usages. A insi plusieurs sortes de fécules du commerce ont celte 
provenance; la plus commune de toutes, celle de la Pomme de terre, n'a pas une autre origine. Les tubercules de cette plante sont des tiges souterraines dont la moelle énormément développée est remplie de fécule. Le papier de riz, d'une texture si fine et d'une éclatante blancheur, qui nous vient de Chine, et qui est employé à la fabrication de fleurs artificielles ou de petites images peintes, n'est. autre chose que des lames de moelle d'une plante de ce pays, l'Aralia papyrifera.

Développement et Accroissement de lá Tige.

Développenent de la tige. - Pour compléter les notions précédentes el avoir une idée d'ensemble de la composition d'une tige dicotylédone, il reste à voir comment se développent les parties qui la constituent.

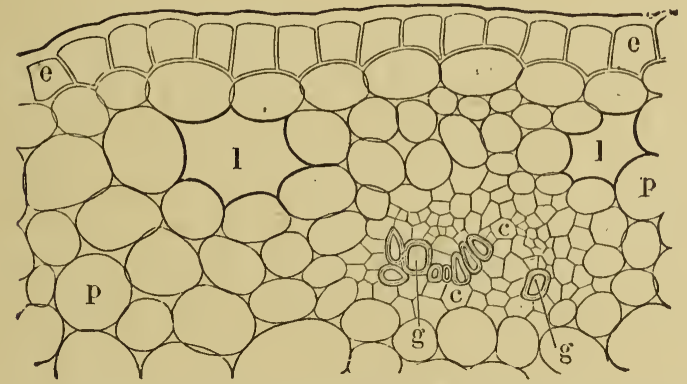

Fig. 52. - Portion d'une coupe transversale du sommet d'une tige (Rhipsalis) (gross. 300); $e$, épiderme surmonté de la cuticule; $p$, parenchyme herbacé; $c$, cellules prosenchymateuses ou fibres; $g$, vaisseaux; $l, l$, lacunes intercellulaires.

Si l'on prend un rameau en voie de formation et qu'on y pratique une coupe transversale (fig. 52), on remarque qu'il se compose d'abord de cellules ayant toutes uniquement le même aspect. Puis, celles de la périphérie 
s'aplatissent et prennent peu à peu les caractères d'un épiderme (e); au-dessous de celles-ci se reconnaît une zone

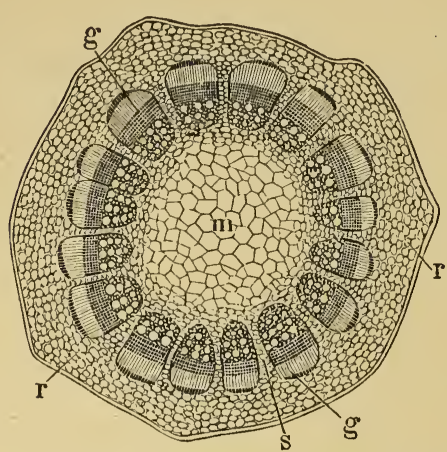

Fig. 53, A. - Coupe transversale d'une jeune tige de Dicotylédone (Kerria japonica). En dedans de l'épiderme et du parenchyme herbacé $(r)$ se voient de nombreux faisceaux $(g)$ encore isolés les uns des autres, disposés sur une ligne circulaire et présentant chacun le liber en dehors, puis le cambium ou zone génératrice, enfin des vaisseaux en dedans ; $s$, rayons médullaires; $m$, moelle. de cellules assez irrégulières et entremêlées de méats $(l, l)$ : c'est le parenchyme cortical. Plus en dedans apparaissent régulièrement disséminées dans la masse cellulaire, sur un même cercle, de petites taches plus sombres qui sont les faisceaux fibro-vasculaires (fig. 52; 53, A et $53, \mathrm{~B})$; leur forme est celle d'un triangle dont la pointe est tournée du côté du centre; la partie extérieure de chacun d'eux est formée de fibres, première indication $\mathrm{du}$ liber, tandis que la partic centrale comprend des fibres et des vaisseaux, ceux qui occupent l'extrême pointe du triangle étant des trachées déroulables; ainsi débutent les faisceaux du bois proprement dit. Entre ces deux ordres de faisceaux du liber et du bois, se trouve une étroite zone génératrice, produisant du bois du côté interne, du liber du côté externe, et destinée à former de nouveaux faisceaux intercalés entre les faisceaux primitifs qui viennent d'être étudiés.

Il peut arriver que le cambium se transforme complètement en fibres ou en vaisseaux; dès lors, le faisceau ne pouvant plus s'accroître est dit fermé (fig. 577), par opposition à celui dans lequel le cambium persiste et tend à augmenter continuellement l'épaisseur du faisceau fibro-vasculaire qui mérite le nom d'ouvert (fig. 53, B). Le premier cas, qui se rencontre exceptionnellement chez les Dicotylédones, est général chez les Monocolylédones el les Cryptogames. 
Les petites masses fibro-vasculaires sont d'abord séparées par de larges espaces de tissu cellulaire, qui diminuent peu à peu par suite de la formation de nouveaux faisceaux

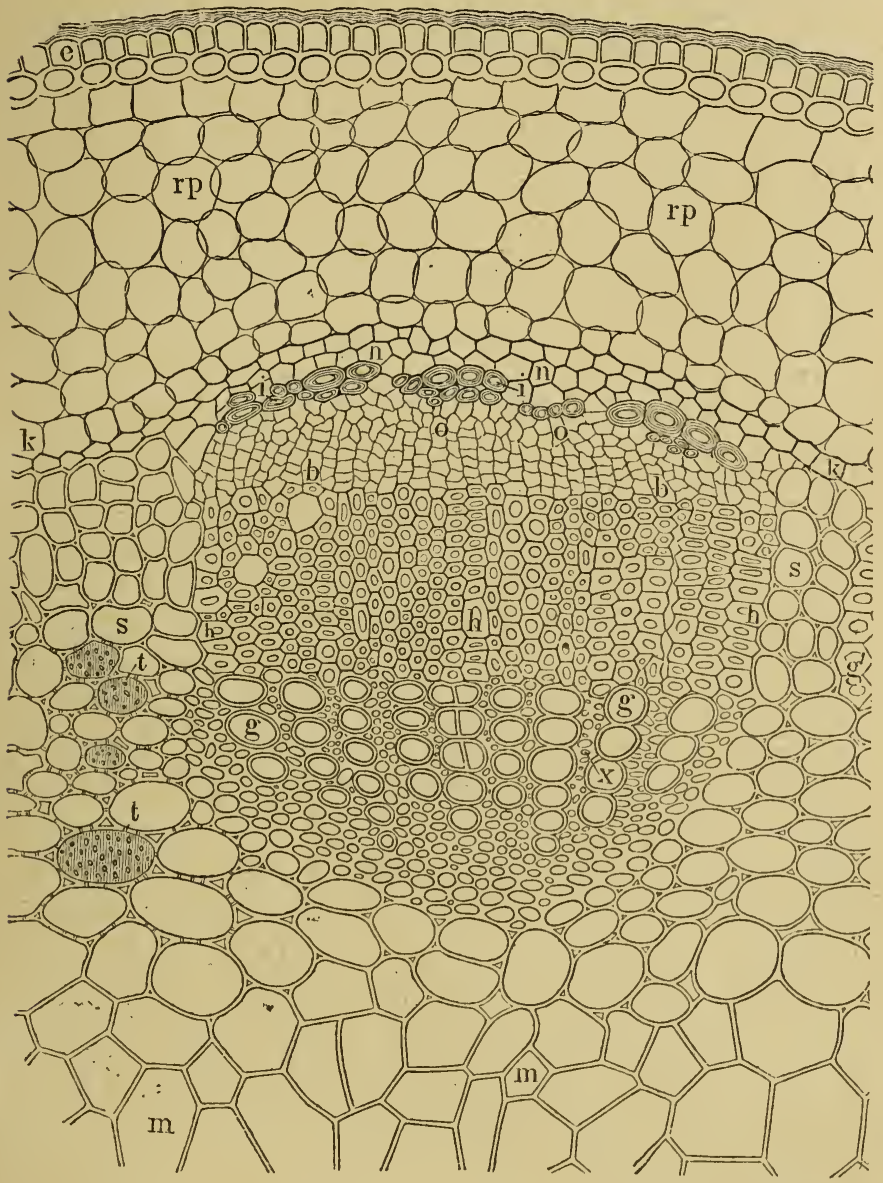

Fig. 53, B. - Coupe transversale d'un faisccau libéro-ligncux très grossi de la fig. 53, A; $e$, épiderme, recouvert d'une cuticule épaisse ; $r p$, parenchyme herbacé; $k$, assise de cellules formant une zone de séparation entre ce parenchyme et le faisceau libéro-ligneux; $n, i, o$, éléments fibreux et cellulaires de la partie libérienne du faisceau; $b, b$, cambium; $h, g, x$, fibres et vaisseaux de la partie ligneuse du faisceau; $m, m$, moelle; $s, s$, prolongements de la moelle entre les faisceaux, origine des rayons médullaires. 
et qui, de plus en plus refoulés, finiront par ne pliss former que d'ćtroites traînées de cellules rayonnant du centre à la périphérie, lesquelles ne sont autres que les rayons médullaires (fig. $53, A$, et $53, B, s$ ).

Enfin tout à fait au centre se voit la moelle $(\mathrm{m})$, d'abord tormée de cellules qui ne se distinguent pas du reste du tissu cellulaire, mais qui prend un peu plus tard, dès la fin de la première année, les caractères que nous lui avons vus sur une tige plus âgée.

Accroissemert en largeur.

Accroissement de la tige. Bourgeon terminal et ÉlongaTION DE LA TIGE. - La tige s'accroît en même temps en deux sens, à savoir, suivant son diamètre transversal el suivant sa longueur. Celle qui n'a qu'une duréc d'un an produit soil accroissement en largeur, comme il a été dit au paragraphe précédent. Mais pour celle qui vit plusicurs années, l'ćlargissement est dû à l'activité du cambium qui, comme on l'a vu (p. 64), produil chaque année une nouvelle couche de liber en dehors, une nouvelle couche de bois en dedans, de sorte que la zone ligneuse forméc la première année se trouve recouverte par une zone nouvelle qui, l'annéc suivante, sera à son tour recouverte par une troisième. Le nombre de ces zones concentriques permet même de reconnaître sur un arbre scić transversalement l’âge qu'il doit avoir.

Arcroissement en hauteur

Quaut à l'accroissement en hauteur ou à l'élongation de, la tige et des rameaux, il dépend du développement des bourgeons foliaires qui terminent ces parties.

Les bourgeons des plantes sont de deux sortes: les uns, ovoïdes renflés, volumineux, produisent des fleurs (bourgeons florifères); les autres, ovoïdes allongés, plus grêles, produisent des feuilles (bourgeons foliifères ou foliaires); c'est de ces derniers seuls qu'il est question en ce moment.

Un bourgeon foliaire n'est autre chose qu'une tige très raccourcie (fig. 54, II); portant des appendices, ou feuilles, trìs rapprochés les uns des autres; le tout est souvent protégé par des écailles, surtout dans les pays froids ou tempérés. Très fréqueinment, ces écailles, sortes de feuilles 
avortées, de consistance dure, ligneuse, sont enduites d'un vernis ou garnies d'une épaisse couche de duvet, pour mieux garantir du froid les parties

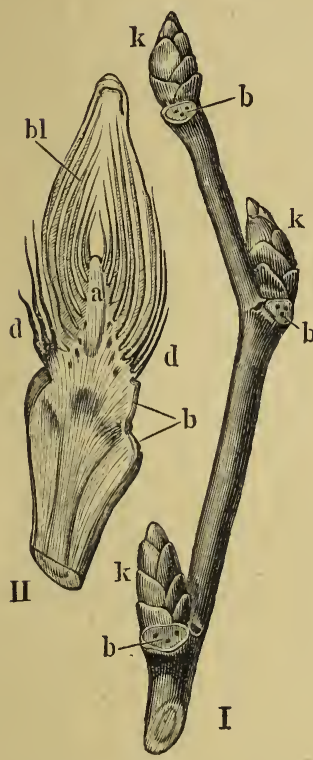

Fig. 54.-I, Une branclie d'Ormeau avec bourgcons (gr. nat.); II, coupe longitudinale d'un bourgeon. Dans les deux dessins : $k$, bourgeon; $b$, cicatrices foliaires; $a$, point ou sommet végétatif; $b l$, jeunes feuilles; $d$, écailles.

délicates qu'elles enveloppent. Ces bourgeons, en effet, doivent passer l'hiver dans un état de non activité pour se développer seulement au printemps suivant. Les feuilles rudimentaires qui entrent dans la constilution des bourgeons ont une disposition constante pour une espèce de plantes donnée, souvent même pour un même genre ou une même famille; et ces caractères, désignés sous lenom de préfoliation, sont employés à la classification. Elles peuvent, en effet, être pliées dans le sens de leur longueur ou de leur largeur, plissées à la manière d'un éventail, roulées sur elles-mêmes de différentes façons, elc.

Par l'accroissement et la division de ses cellules, le cône terminal de la lige (fig. $54, I I, a)$ s'allonge et produit au fur et à mesure de nouvelles feuilles sur ses parties latérales, de sorte qu'il ne se trouve jamais à nu, mais au contraire toujours enveloppé de feuilles qui le recouvrent complètement. En même temps, les feuilles de la base du bourgeon s'épanouissent, et l'espace qui sépare deux feuilles superposées constitue ce qu'on appelle un entre-nœud. Il peut se faire que les entre-nœuds une fois dégagés du bourgeon ne s'allongent pas, et les feuilles restent alors très serrées sur la tige. Plus souvent, chaque entre-nœud s'allonge, alor's les feuilles sont plus espacées, et la tige s’accroît 
d'autant ; cette croissance intercalaire, comme on l'appelle, vient donc ajouter ses effets à la croissance terminale, dont il a été précédemment question.

La pousse nouvelle qui résulte de cette évolution du sommet de la tige a une composition identique à celle qui a été indiquée dans l'étude faite sur une tige d'un an, c'està-dire,que l'on trouve: au centre, la moelle, sous forme d'un étroit cylindre dont la hauteur est celle de la nouvelle pousse, puis, en dehors d'elle une couche ligneuse, prolongement de celle qui s'est formée sur toute la longueur de la tige pendant cette même période végétative et qui dépasse, on le comprend, de toute la hauteur de la pousse, la couche ligneuse. de l'année précédente. Il en est de même pour la couche corticale formée en dehors de la zone ligneuse; il faut remarquer seulement, que la nouvelle formation corticale, dans la partie de la tige située au-dessous de la pousse récente, occupe la portion interne de l'écorce dont elle a repoussé en dehors toutes les couches précédemment formées.

\section{TABLEAU RÉSUMÉ DE LA STRUCTURE DE LA TIGE}

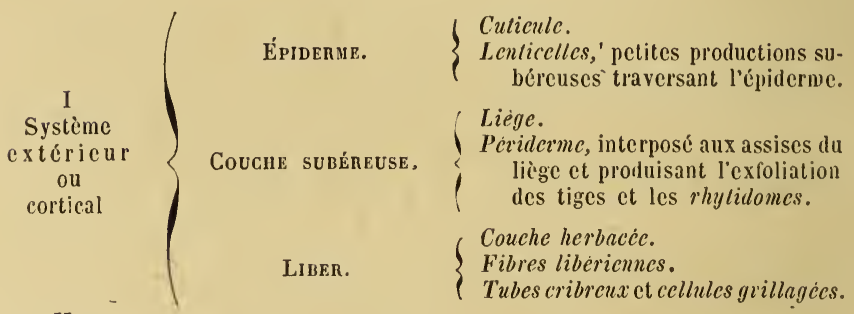

II

Zone génératrice ou Cambium, produisant le bois en dedans, le liber en dehors.

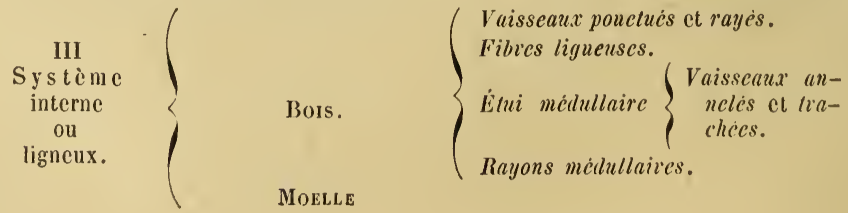


Modifications dans la structure de la tige présentées par certaines Dicotylédones. - $1^{\circ}$ Conifères. Les arbres ver's, résineux, qui constituent le groupe des Conifères,

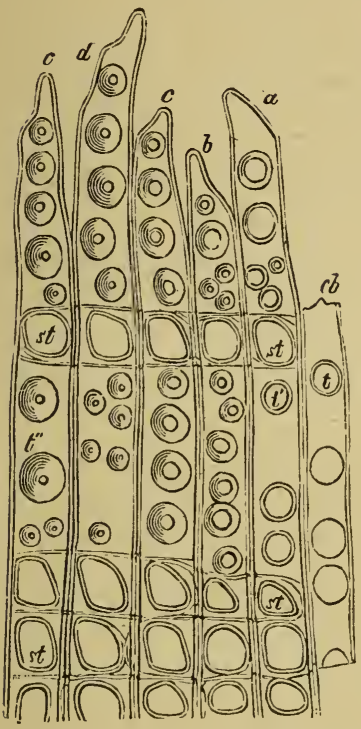

Fig. 55. - Fibres du bois des Conilères; $a, b, c, d$, extrémités des fibres; $c b$, une fibre briséc ; $s t$, $t, t^{\prime}$, ponctuations simples ; $t^{\prime \prime}$, ponctuations aréolécs. tels que les Pins, Sapins, Cèdres, Cyprès, etc., présentent dans la composition du bois des particularilés importantes.

Toute la masse de ce dernier est dépourvue de vaisseaux ; ceux-ci n'occupent que la région de l'étui médullaire et sont annelés et spiralés. Par contre, le corps ligneux est formé en grande partie de ces fibres à ponctuations aréolées (fig. כ5) dont il a été question plus haut, p.11. Ajoutons que les rayons médullaires sont extrêmement étroits et ne se composent, le plus souvent, que d'une seule ligne de cellules.

$2^{\circ}$ Lianes. Ces plantes qui habitent surtout les pays chauds et dont l'aspect est tout particulier, sont réparties en plusieurs familles naturelles qui présentent chacune des particularités de structure extrêmement singulières. Il nous suffira d'en citer ici quelques-unes. Ainsi celles qui forment le groupe des Gnétacées, au lieu d'avoir le liber disposé en une zone unique, le présentent réparti en autant de zones qu'il y a de couches annuelles de bois. Dans les Bignoniacées, l'écorce se produit avec une plus grande activité aux extrémités de deux diamètres se coupant en croix, et s'enfonce comine autant de coins dans l'épaisseur du bois, ce qui donne un aspect fort singulier à la coupe transversale de la tige. 
B. Monocotylédones.

Étude d'une coupe transversale.

Système cortical.

Parties constitutives de la tige. - La coupe transversale d'une plante monocotylédone, d'une tige de Palmier, par exemple, offre un aspect tout différent de celui que nous connaissons à la tige des Dicotylédones.

On voit à l'extérieur une zone corticale peu épaisse, et en dedans un corps ligneux, dans lequel on ne reconnaît pas de zones concentriques et qui ne présente ni rayons médullaires, ni étui médullaire, de même qu'il n'y a pas de moelle centrale bien définie.

Le système cortical offre un épiderme, un hypoderme, un parenchyme cortical ou couche herbacée.

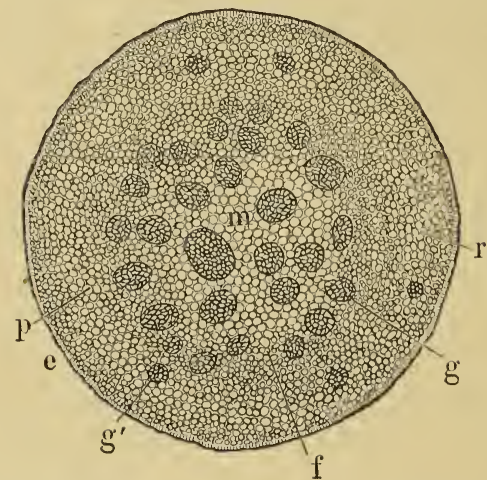

Fig. 56. - Coupe transversale d'une jeune tige de Monocotylédone (Aspidtslra) (gross. 15); $e$, épiderme; $p, r$, tissu cellulaire; $m$, moelle; $g, g$ ', faisceaux disséiinés ; $f$, tissu médullaire interoposé aux faisceaux.

Le système ligneux se montre formé d'une masse cellulaire dans laquelle paraissent disséminés un assez grand nombre de faisceaux fibro-vasculaires distincts, bien plus nombreux et rapprochés les uns des autres vers la périphérie, bien plus espacés et plus rares vers le centre, de 
sorte que, contrairement à ce qui a lieu dans les Dicotylédones, la partie extérieure est d'un tissu beaucoup plus dur que le cœur de la tige.

L'étude d'un faisceau isolé (fig. 57) montre $1^{\circ}$ que sa forme est à peu près arrondie et qu'il est composé, dans sa partie périphérique, de fibres à parois épaisses formant autour de lui un cercle complet, qui représente le liber; $2^{\circ}$ qu'en dedans est une petite masse formée de vaisseaux ponctués ou rayés mêlés de fibres ligneuses, puis de vaisseaux annelés, et_enfin tout à fait au centre, de vaisseaux spiralés ou trachées; $3^{\circ}$ qu'entre ces deux ordres d'éléments, fibres et vaisseaux, se trouve une couche de forme annulaire, le cambium, qui a servi à les former, mais dont la période d'activité est courte, de sorte qu'au lieu de continuer à produire des vaisseaux et des fibres ligneuses, d'une part, des fibres libériennes, de l'autre, il s'entoure promptement de tous côtés d'éléments définitifs $(g)$ et n'a plus aucune communication avec les rayons médullaires et la zone génératrice des faisceaux voisins; il en résulte que le faisceau ne peut augmenter de volume et mérite bien le nom qu'on lui donne, de faisceau fermé. I tes faisceaux ne s'épaississant pas, le tronc de la plante reste toujours assez grêle relativement à sa hauteur. Tels sont les renseignements que l'on peut obtenir par l'examen des coupes transversales.

Une coupe longitudinale montre la façon dont ces mêmes faisceaux se comportent dans leur trajet à l'intéricur de la tige (fig. :8). On peut observer $1^{\circ}$ que chacun d'eux aboutit à une feuille et que le nombre des premiers est exactement le même que celui des secondes; $2^{\circ}$ que, commençant en bas, par une extrémité très tenue, chaque faisceau grossit à mesure qu'on le considère à des niveaux plus élevés, disposition qui explique comment il se fait que la tige a sensiblement le même diamètre dans toute sa hauteur; $3^{\circ}$ que chacun d'eux est, à sa partie inférieure, d'autant plus voisin du centre de la tige, que la feuille à laquelle il aboutit est à un niveau moins élevé, par conséquent moins récemment fornée; $4^{\circ}$ enfin, que le trajet du faisceau, au lieu d'être rectiligne, est flexueux, de sorte

Structure des faisccaux.
Étude d'une coupe longitudiuale. 
qu’à mesure qu'il remonte, il s'incline d'abord vers la partic centrale de la tige, pour s'infléchir ensuite dans le sens opposé el finir par traverser obliquement les tissus afin d'aboutir à la feuille correspondante.

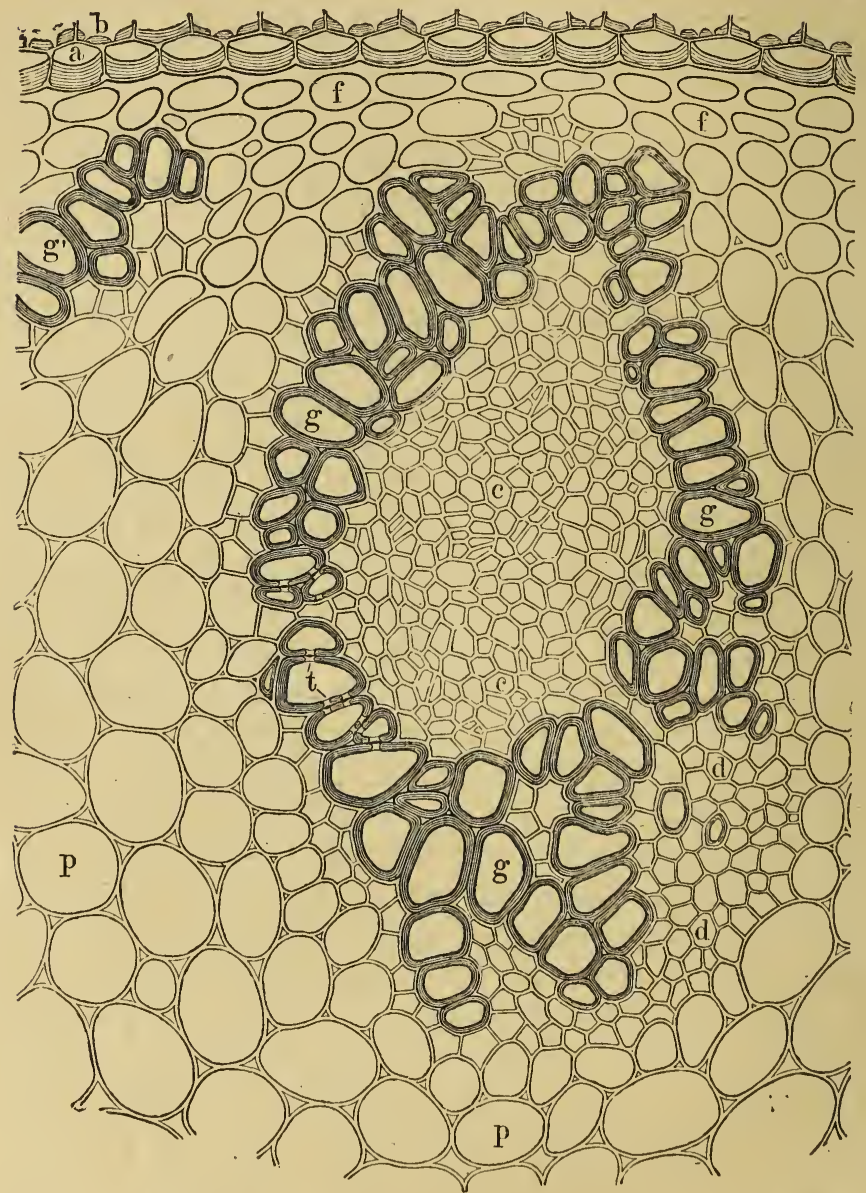

Fig. 57. - Coupe transversale d'un faisceau fermé de Monocotylédone (Muguet) igr. 450);g. cellules a parois très épaissies ou sclérenchymatcuses entourant le faisceau qui est ainsi complìtement fermé et ineapable de s'élargir; $t$, perforations dans les parois de cellules voisines; $c$, éléments du faisceau; $p$, tissu cellulaire au milieu duquel se sont développés les faisceaux; $a$, assise périphérique de cellules à parois très épaissies d'un seul côté; $g^{\prime}$, cellules sclérenchymateuses du faisecau voisin. 
Celte disposition, on le voit, est bien différente de celle des Dicotylédones (fig. 59), car chez ceux-ci les parties ligneuses les plus centrales sont les plus récemment formées et

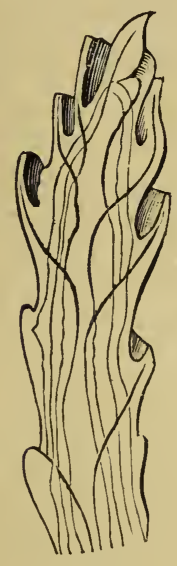

Fig. 58.

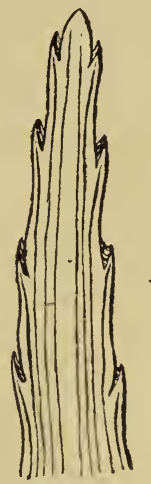

Fig. 59,

Fig. 58. - Marche des faisceaux fibro-vasculaires dans la tige des Monocotylédones.

Fig. 59. - Marche des faisceaux fibro-vasculaires dans la tige des Dicotylédones.

aboutissent par conséquent aux feuilles les plus élevées, qui sont, on le comprend, les dernières épanouies.

L'étude qui vient d'être faite de la structure de la tige des Monocotylédones suffit à expliquer le mécanisme de sa croissance. Celle-ci est très faible dans le sens transversal et due seulement à un léger épaississement des faisceaux isolés, l'élongation se faisant d'ailleurs d̀e la même façon que celle des Dicotylédones.

Modifications dans la structure de la tige présentées par certaines Monocotylédones. - La structure qui vient d'être indiquée pour la tige des Monocotylédones, n'est pas sans offrir, comme on va le voir, quelques exceptions.

$1^{0}$ Dragonniers. Ces plantes, contrairement à ce que nous venons de constater dans la majorité des Monocotylédones, ont une tige d'un diamètre souvent considérable, et nota-

Croissance en hauteur et en largeur. 
blement plus gros à la base qu'au sommet. L'accroissement en épaisseur paraît dû surtout à ce que, indépendanment des faisceaux qui se rendent aux feuilles, il s'en forme à chaque période végétative une autre zone en dehors d'eux, laquelle a pour effet d'augmenter le diamètre de ces arbres. On cite, dans l'île de Ténériffe, des Dragonniers (Dracana) qui ont jusqu'à 6 et 8 m. d'épaisseur, soit 18 à $24^{\mathrm{m}}$. de circonférence, à la base.

2. Graminées. La tige de ces plantes offre quelques particularilés intéressantes; dans presque toutes, le parenchyme central se rompt et se
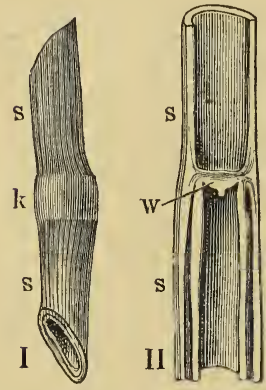

Fig. 60. - Tige fistuleuse de Roseau (Phragmiles communis). $I$, vue extérieure; $k$, nœud ; $s$, $s$, entre-nœud ; 11 , coupe longitudinale; $w$ cloison transversale. détruit par suite de l'amplification des parties extérieures; à sa place se voil un large espace vide, et la tige est dite alors fistuleuse; telles sont celles du Blé et de nos autres céréales, où elles sont connues sous le nom de chaumes. En outre, au niveau de la naissance de chaque feuille, la cavité centrale est fermée par une cloison transversale, une sorte de plancher très résistant, formé par les ramifications des faisceaux fibro-vasculaires qui s'y entrecroisent, disposition qui donne à toute la tige une bien plus grande solidité. Cette particularité anatomique est des mieux caractérisées dans le Bambou (tig. 38), qui appartient à la même famille. Ces cloisons sont assez épaisses et résistantes pour que cette lige, coupée par tronçons dans les entre-nœuds, puisse servir dę vases naturels très solides.

\section{G. Acotylédones.}

Tiges des Cryptoganes cellulaires et des Criptogames vasculaires. - $\Lambda$ u point de vue de leur structure, ces 
plantes, les plus inférieures du règne végétal, se répartissent en deux groupes, les Cryptogames cellulaires et les Cryptogames vasculaires.

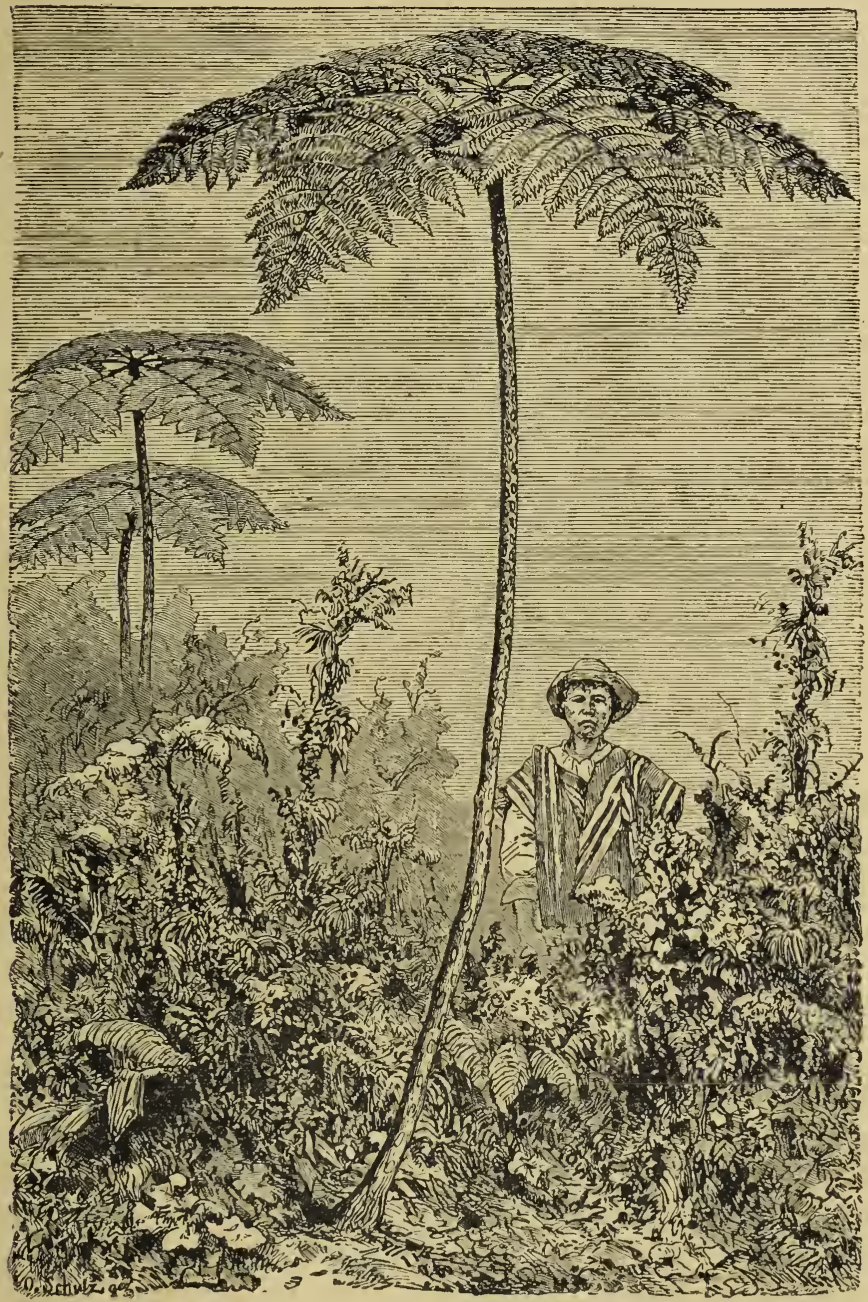

Fig. 61.- Port des Fougères arborescentes. 
Les unes, en effet, sont entièrement formées d: cellules qui peuvent se modifier légèrement, s’allonger plus ou moins, épaissir leurs parois, mais qui ne vont jamais jusqu’à prendre la forme de vaisseaux; tels sont les Lichens, les Champignons, les Algues, les Mousses.

Les autres offrent des éléments vasculaires différemment disposés suivant l'ordre auquel ils appartiennent; ce sont les Lycopodiacées, les Prêles, les Fougères, etc.

Tige des Fougères.

Prenons comme type de la structure des Acotylédones l'une de ces belles Fougères auxquelles leur port a fait donner le nom d'arborescentes. On voit à l'extérieur un

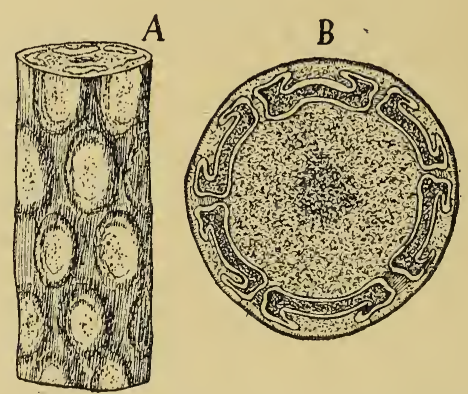

Fig. 62. - Structure de la tige des Fougères; $A$, tronçon de Fougère, sur laquelle se voient les cicatrices des feuilles après leur chute $; B$, coupe transversale de la tige, présentant, en dedans de l'écorce, des faisceaux fibro-vasculaires disposés circulairement et séparés les uns des autres de façon à laisser la moelle, qui forme la plus grande masse de la tige, en communication avec l'écorce.

épiderme, le plus souvent masqué par de larges écailles et marqué par de nombreuses cicatrices ovalaires résultant de la chute des feuilles; puis vient une écorce très résistante, formée, en dehors, de cellules polyédriques, et en dedans, de cellules allongées en fibres. On voit ensuite une zone de gros faisceaux fibro-vasculaires ayant chacun la forme d'un croissant dont les extrémités sont tournées vers l'extérieur. Tous ces faisceaux communiquent entre eux, dans leur course longitudinale, par des anastomoses, de façon à former un cylindre creux, comme treillissé par suite de la présence de fentes étroites ménagées sur sa paroi. 
Chaque faisceau, qui est limité par une zone foncée, noirâtre, de fibres à parois épaisses représentant le liber, offre, dans son centre, des vaisseaux prismatiques scalariformes très remarquables par leur volume.

Les faisceaux sont bientôt arrêtés dans leur développement, de sorte qu'ils méritent, comme ceux des Monocotylédones, le nom de faisceaux fermés (1).

Quant à la portion de la tige située en dedans de la zone des faisceaux, elle consiste en un parenchyme peu résistant, sorte de moelle qui se déchire et se crevasse lorsque la tige vient à se dessécher.

Les Prèles ou Equisétacées offrent plusieurs particularités de structure fort remarquables. Contentons-nous de Tige faire observer que la cuticule épidermique est encroûtée d'une épaisse couche de silice, qui fait employer ces plantes au polissage du bois et de l'ivoire.

(1) Lorsque la plante est jeune, les faisceaux offrent des vaisseaux annelés et spiralés, qui plus tard sont remplacés par des vaisseaux scalariformes. 


\title{
GHAPITRE IV
}

\author{
Racine.
}

Définition. Désignation des racines, $1^{\circ}$ d'après le milieu dans lequel elles vivent; $2^{\circ}$ d'après leurs caractères extérieurs. Radicelles. Racines adventives. Durée des racines. Principales modifications : Crampons, Suçoirs,Tubercules. - Structure de la Racine. Étude du sommet de la racine : la Pilorhize et le Point végétatif. Allongement de la racine. Etude de la racine à un niveau un peu plus élevé : épiderme; poils radicaux ; écorce, etc. - Différences dans la structure de la racine des Dicotylédones, des Monocotylédones et des Acotylédones. Accroissement de la racine en épaisseur.

Définition. - La racine est celle partie de la plante qui, en général, s'enfonce dans le sol pour y puiser les éléments nécessaires à la nutrition du végétal.

De forme d'abord cylindrique, elle est attachée par sa base à la tige dont elle prolonge l'axe, et va en s'effilant en cône à son sommet. Mais assez souvent, au lieu de continuer l'axe de la tige, elle part de ses parties latérales, de ses rameaux, parfois même d'une feuille.

DÉnomination des racines. - 10 D'après le milieu dans lequel elles vivent, on peut classer les racines en souterraines, de beaucoup les plus nombreuses; en aériennes, comme celles des Orchidées (voyez page 84, ce qui est dit pour les racines adventives); en aquatiques, comme celles des Lemnas ou Lentilles d'eau (fig. 68) et de quelques autres végétaux ; en épiphytes, comme celles du Gui, de la Guscute et de plusieurs autres espèces qui vivent en parasites sur diverses plantes.

$2^{\circ}$ La forme des racines et le mode de ramification servent encore plus souvent de base à leur classification. 
La racine primitive, celle qui continue directement la tige, reste ordinairement pendant longtemps, souvent même pendant toute la vie de la plante, prédominante sur celles qui

A

B

D

C
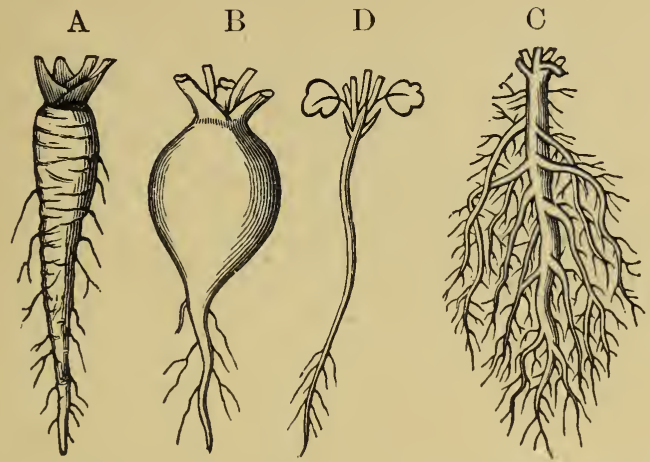

Fig. 63. - Différentes formes de racines ; $A$, racine pivotante conique (Carotte); $B$, racine napiforme (Navet); $C$, autre forme de racine pivotante, le pivot reste parfaitement distinet au milieu des racines secondaires; $D$, racine filiforme

se développent ensuite, et porte le nom de pivot; en pareil cas, la racine est désignée sous le nom de pivotante. L'accroissement, l'épaississement de la racine primitive peut se faire de différentes façons : s'il s'accuse davantage à la base de l'organe, pour aller en diminuant à mesure qu'on se rapproche de l'extrémité, la racine devient conique (Carotte, Belterave); l'épaississement devenant encore plus considérable à la base, et la racine étant peu allongée, de façon à ressembler à une toupie, comme dans le Navet, elle est dite napiforme; si l'épaississement a lieu seulement ver's le milieu de la longueur de la racine, elle devient fusiforme ou tubériforme comme celle du Dahlia (fig. 64).

Dans un assez grand nombre de cas (Cryptogames vasculaires, beaucoup de Monocotylédones, un certain nombre de Dicotylédones), la racine primitive ne s’épaissit pas; elle se détruit ou reste mince tout en s'allongeant, ce qui lui vaut le nom de filiforme (fig. 63, D).

D'autre part, il peut se faire que la racine primitive cesse de s'accroître, tandis que les racines secondaires se déve- 
loppent beaucoup et rampent ordinairement à une faible profondeur au-dessous du sol; c'est ce qu'on appelle une racine fibreuse ou chevelue (fig. 65).

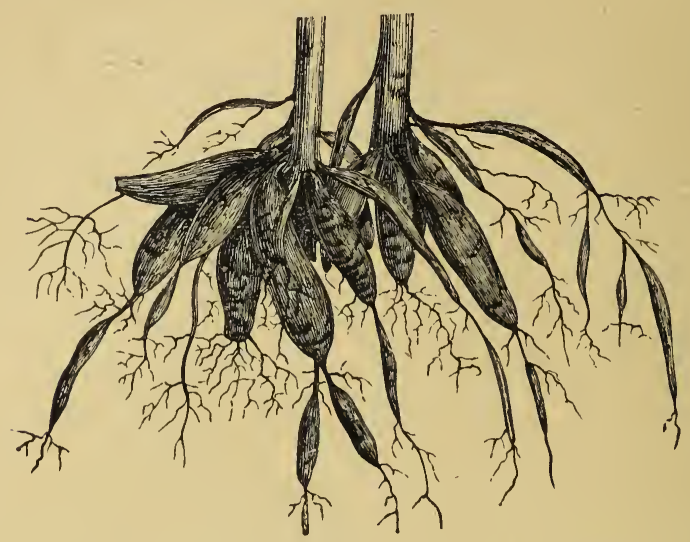

Fig. 64. - Racines fusiformes ou tubériformes du Dahlia.

Radicelles. - On entend par ce terme les racines qui se produisent secondairement, c'est-à-dire sur le pivot, surtout quand elles sont encore jeunes et grêles.

Leur naissance ne se fait pas au hasard, mais avec régularité,de façon à être disposées les unes au-dessous des autres, en un certain nombre de séries verticales qui, suivant les espèces, varie de 2 à 20 ou 30 et plus, selon la grosseur de la racine primitive. Le nombre est à peu près constant pour les plantes d'une même famille naturelle ; ainsi on compte deux séries de radicelles dans les Crucifères, les Papavéracées, etc., quatre dans les 0 mbellifères, Labiées, Malvacées, etc.

La loi qui préside à la disposition des radicelles sur le pivot s'applique également aux radicelles qui naissent des racines secondaires, tertiaires; il en est de même pour toutes les autres.

Racines abventives. - Il a été dit au commencement du chapitre que des racines se montrent assez souvent, en 
dehor's des régions normales, sur les parties latérales de la tige, les rameaux ou les feuilles : d'où leur nom de racines adventives. Mais il faut remarquer, que même dans ces cas, elles ne naissent pas toujours indifféremment en tel ou tel point.

A

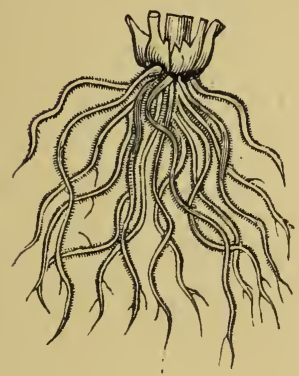

B

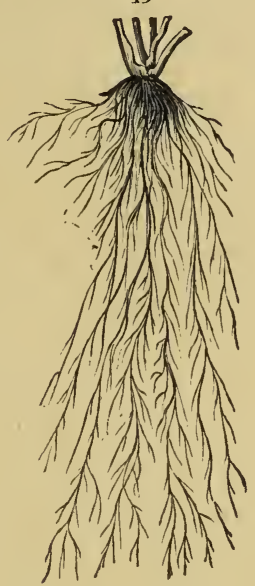

Fig. 65. - Racines fibreuses de Gramincé $(A)$ et de Légumineuse $i B)$.

Ainsi sur certaines Aroïdées, à chaque feuille se trouve régulièrement opposée une racine; dans les Prêles, au-dessous de chaque verticille foliaire se voit un verticille de racines.

Les racines adventives se montrent à des hauteurs variables sur la tige, le plus souvent à la base même, au contact du sol, dont l'humidité suflit à provoquer leur apparition. C'est sur la production adventive des racines que sont fondées les importantes pratiques connues en horticulture sous les noms de bouturage et de marcottage. La première opération consiste à détacher un rameau et à l'enfoncer dans la terre humide; des racines ne tardent pas à se développer à l'extrémité inférieure et suffisent bientôt à nourrir cette nouvelle plante. Dans le marcollage, on couche d'abord un rameau ou on le met d'une façon ou de l'autre en contact avec le sol humide; bientôt des racines se développent, et 
on sépare la branche, qui peut désormais se suffire à cllemême.

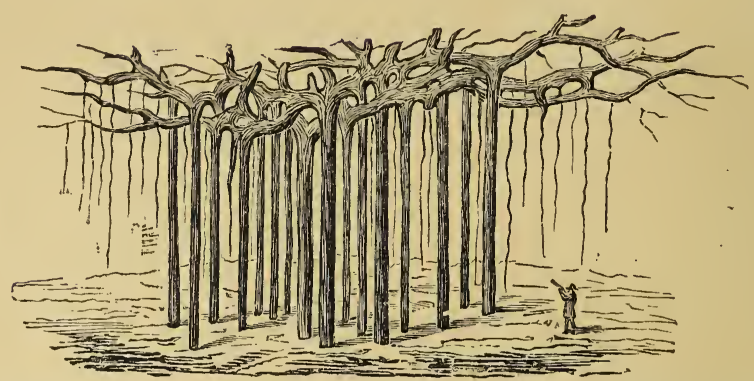

Fig. 66. - Racines adventives du Gommier; les unes sont volumineuses et profondément enfoncées dans le sol; les autres, minces, ne l'ont pas encore atteint.

Durée des racines. - Tandis que le plus souvent la durée de la racine est celle de la plante elle-même, annuelle, bisannuelle ou vivace, il est un certain nombre de cas où la racine principale et les racines secondaires se détruisent, sans que pour cela la plante périsse; c'est qu'alors des racines adventives se sont formées sur la tige à des hauteurs variables, puis enfoncées dans le sol et pourvoient à la nutrition du végétal. Les plantes à tige rampante souterraine, comme le Muguet, ou à la surface du sol, comme le Fraisier, le Lierre, nous en offrent des exemples: à mesure que leur tige se déplace par le fait de leur croissance (voy. page 5̋3), les anciennes racines meurent et de nouvelles se produisent en d'autres points. Certains arbres, le Figuier des Banians, le Gommier, entre autres, émettent de leurs branches horizontales de nombreuses racines adventives qui finisssent par atteindre le sol et fout l'effet d'autant de tiges secondaires qui soutiennent ces grands arbres et les nourrissent alors que les racines primitives sont détruites (fig. 66).

Modifications de la racine. - Crampons. Ce sont des racines de forme spéciale, au moyen desquelles certaines plantes grimpantes, comme le Lierre, se fixentaux murs 
ou autres objets voisins. Mises au contact du sol, elles s'y développent en racines ordinaires, comme on le voit quand on emploie le Lierre à faire des bordures.

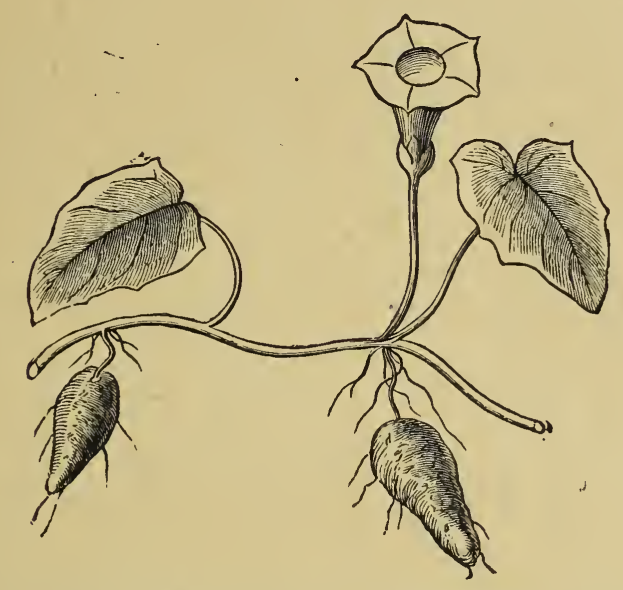

Fig. 67. - Portion d'une tige de Batate (Convolvulus balalas avec ses racines tubcrculcuses.

Suçoirs. Ce sont des racines que développent certaines plantes quand elles se trouvent au contact de certains autres végétaux. Elles enfoncent leurs racines dans les tissus mêmes de leur hôte et en pompent les sucs nutritifs. La Cuscute se comporte ainsi envers le Trèfle, la Luzerne; le Gui agil à peu près de même. (Voy. Chap. xıI, Parasitisme.)

Tubercules. Certaines racines s'arrêtent dans leur croissance et se gorgent de fécule, en prenant une forme arrondie. C'est une réserve nutritive qui sera employée dans la période de végétation suivante; le Dahlia, déjà cité, nous en offre un exemple. La figure 67 montre les racines, renflées en tubercules, de la Batate comestible. Les Orchis en offrent un autre cas bien remarquable : leur racine se compose de deux tubercules, dont l'un, déjà flétri, a épuisé sa réserve nutritive au profit de la tige qui a produit les feuilles et les fleurs de l'année; l'autre est plein de fécule et sera bientôt 
utilisé par la plante; or ce tubercule n'est autre chose qu'une réunion de plusieurs racines coalescentes, c'est-àdire, fondues en quelque sorte en une masse unique.

\section{STRUCTURE DE LA RAGINE}

Etude nu sommet de la bacine. Croissance de la bacine EN Longueur. - Pour bien se rendre compte de la disposition des tissus de la racine, il faut considérer celle-ci dans ses parties les plus jeunes, c'est-à-dire près de son extrémité. Cette région est toujours, en effet, la plus récemment formée, car la racine ne s'allonge pas indifféremment par tous ses points, mais par cette seule extrémité, laquelle

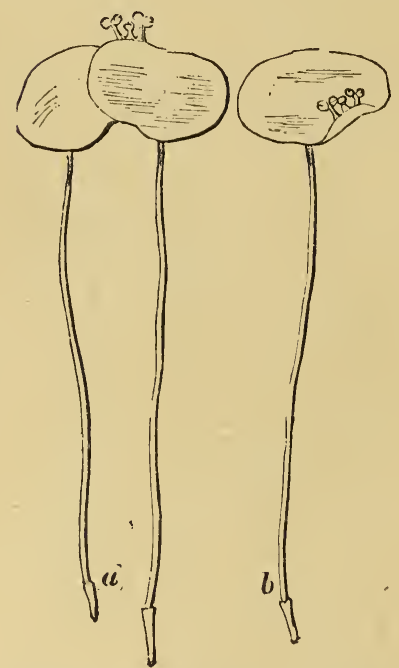

Fig. 68. - Lemnas avec leurs racines flottantes dont l'extrémité offre une coiffe ou pilorhize $(a, b)$. renferme ce qu'on appelle le point végétatif. C'est donc là que l'on doit porter son attention pour étudier la structure de la racine à son étal de plus grande simplicité.

Avant de pénétrer dans l'épaisseur des tissus, au moyen d'un instrument tranchant, si l'on examine l'extrémité bien intacte d'une racine(cet examen est surtout facile sur certaines plantes aquatiques dont la racine est flotlante, les Lemnas ou Lentilles d'eau, fig. 68, par exemple), on voit que sa fine pointe est enveloppée d'une sorte de petite coiffe ou pilo-

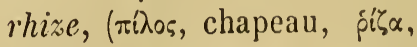
racine), formée de cellules assez résistantes, qui servent à 
protéger la partie sous-jacente, laquelle constitue le point végétatif. Cette petite coiffe, formée de quelques rangs de

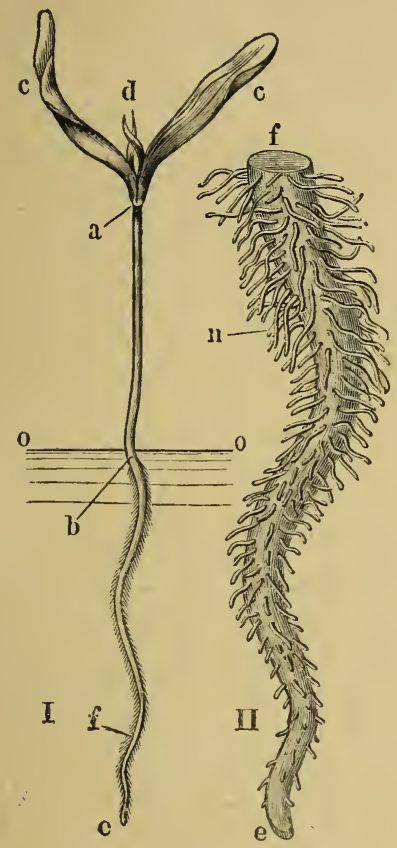

Fig. 69. - Plante en voie de développement (Érable); I, ensemble de la plante (grandeur naturelle); $I I$, extrémité de la racine (grossie 6 fois); $-a b$, tige; $b e$, racine garıic de poils; $f e$, lieu de l'allongement de la racine; $c, c$, feuilles cotylédonaires; $d$, bourgeou ou gemmule; $n$, poils radicaux; 0 o, surface du sol. cellules superposées, est un produit de l'extrémité de la racine; elle s'exfolie et se désagrège par l'extérieur, à mesure que de nouvelles couches sont produites à sa partie interne.

Le point végétatif de la ra- Point végétatif cine (fig. $70, I, i$ ), recouvert et allongement par la pilorhize, se compose uniquement de cellules à pitrois très minces, gonflées de protoplasma et douées, par conséquent, d'une grande activité; elles se multiplient continuellement par segmentation, pour produire de nouveaux tissus, d'où résulte l'allongement de la racine, laquelle ne s'accroît que par son extrémité même, comme on peut s'en assurer par une expérience bien simple, qui consiste à marquer une jeune racine de Haricot, par exemple, de lignes de couleur, distantes les unes des autres d'un centimètre, à partir de la pointe. On arrive ainsi à constater facilement que l'allongement ne se fait qu'au niveau du premier centimètre ainsi délimité.

Étude de la racine a un Niveau un peu plus Élevé. Un peu plus haut, les choses changent: on voit les cellules, naguère toutes semblables, se différencier en éléments de 
formes diverses. Une coupe transversale pratiquée dans celle région va nous les faire connaître.

Épiderme. On trouve d'abord, en allant de dehors en dedans un épiderme, qui se détruit promptement et ne se reconnaît plus sur les parties quelque peu âgées (1). Cet épiderme offre assez souvent une cuticule, comme celui de la tige; mais il présente surtout des organes d'une grande importance, ì Poils radicaux. savoir, des poils,groupés surtout vers l'extrémité de la racine, au-dessus de la pilorhize et que l'on a appelés suçoirs, en raison du rôle qu'ils doivent jouer dans la nutrition de la plante, car ce sont eux qui pompent dans le sol humide les liquides nourriciers. Ils sont ordinairement simples et unicellulaires, quelquefois ramifiés et pluricellulaires. Leur existence est éphémère; mais à mesure que les premiers formés tombent, il s'en développe d'autres vers l'extrémité de la racine qui continue à s'allonger.

Écorec. Les autres parties de l'écorce, à part le liber, se trouvent disposées comme celles de la tige; on y trouve donc, plus ou moins développés, une couche subéreuse et un paren. chyme cortical ou couche herbacée; seulement la chlorophylle manque d'ordinaire dans la racine; cependant il en existe dans un certain nombre de plantes. Quant au liber, il est disposé de telle façon qu'il parait appartenir bien plus au bois qu'à l'écorce.

Bois. En effet, on voit en dedans des parties précédentes (fig. $70, I I I)$, répartis sur deux cercles concentriques, des faisceaux de fibres libériennes $(b)$ et de vaisseaux ligneux $(g)$, de façon que l'un des premiers se trouve toujours compris entre deux des seconds, au lieu d'être placés sur leur côté extérieur, comme cela se voit dans la tige. Les faisceaux vasculaires se développent à la partie

(1) Dans la plupart des cas, les cellules épidermiques ne forment qu'une assise; mais dans les remarquables racines adventives des Orchidées on compte plusieurs assises superposées, tont les plus intéricures offrent les caractères ordinaires des cellules épidermiques, tandis que les racines extérieures sont remplies de gaz, qui leur donnent une teinte blanche, souvent brillante. Cel épiderme compliqué a reçu le nom de voile. 
interne d'une zone génératrice (s) qui laisse en dehors d'elle les faisceaux libériens. (La lettre $s$ de la fig. 70, 111, devrait être placée en face des groupes de vaisseaux.)
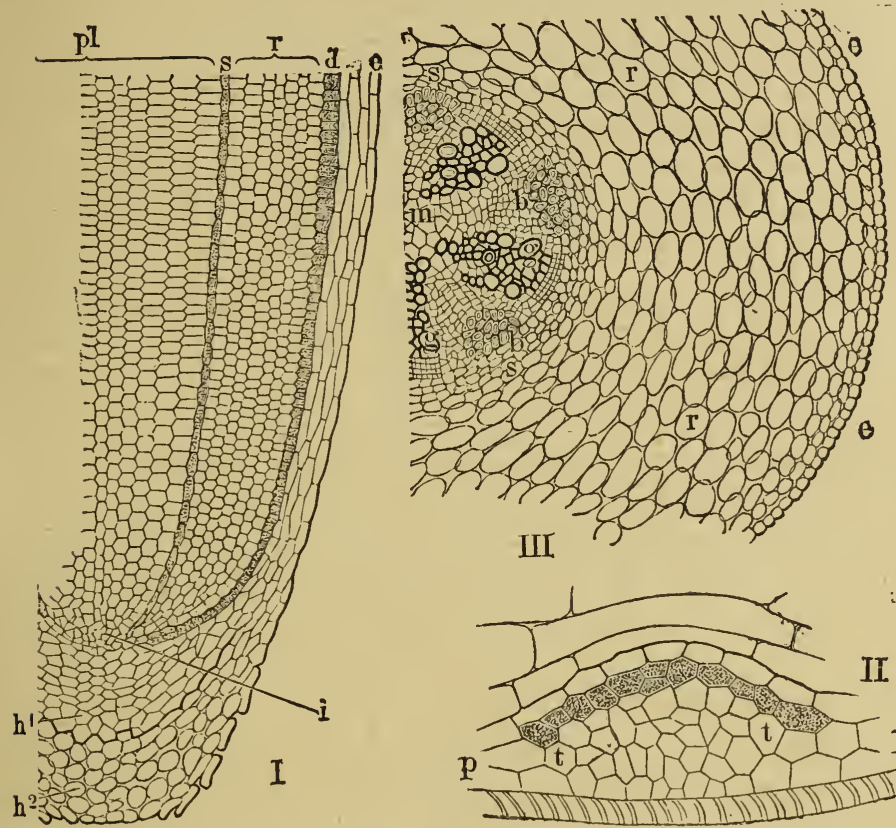

Fig. 70. - Structure de la racine de Fève; I, coupc longitudinale de l'extrémité de la racine (voy. note 1 ); $I I$, coupe passant par l'axe d'une racine secondaire en voie de formation sur la racine principale : ses tissus propres $(\iota, p)$ refoulent devaut cux les tissus de la racine principale, qu'ils finiront par traverser; $I I I$, coupe transversale d'une portion de jeune racine d'un Liseron (Ipomøa), montrant déjà les tissus définitifs. $-e$, épiderme; $r$, parenchyme cortical; $s$, péricambium ou zone génératrice; $b$, faisceaux fibreux alternant avec les faisccaux vasculaires $(g) ; m$, moclle.

\section{Le nombre des faisceaux, toujours peu considérable,}

(1) Dans la partic de ía racine en voie de formation, par conséquent à son extrémité, les tissus n'ont pas encore leur forme définitive et sont désignés par des noms spéciaux qui vont être indiqués; voyez à ce sujet la fig. $70, I: e$, assises de cellules formant la coiffe ou pilorhize $\left(h^{1}, h^{2}\right)$, et produites par la zone $d$, appelée pour cela dermatogène, qui produit aussi l'épiderme et les poils radicaux; $r$, parenchyme cortical; $s$, procambium, jouant le rôle du cambium déjà étudié dans la racine; $p l$, plérome ou còne central, qui se transformera en moelle, bois et liber; $i$, sommet végétatif, point de départ de tous les tissus, par division successive de ses cellules. 
répond à celui des séries de radicelles, lesquelles naissent sur la racine, conslamment en face des vaisseaux, du moins chez les Phanérogames (voy. p. 84); si les radicelles sont disposées sur deux séries verticales, on ne compte que deux faisceaux vasculaires, lesquels répondent chacun à une série; si les radicelles sont disposées sur $\mathbf{3}, 4, \boldsymbol{y}$ séries verticales, les faisceaux sont au nombre de 3,4 ou 5 .

Les fibres de ces faisceaux ont leur paroi épaisse mais peu résistante, comme d'ailleurs celles de l'écorce de la tige. Quant aux vaisseaux, les uns sont ponctués, d'autres rayés, etc.; il en est aussi de spiralés, mais la spiricule n'est pas déroulable. Un fait important doit être noté, c'est que les vaisseaux se forment en allant de dehors en dedans, de sorte que les plus récents sont les plus près du centre de la racine, disposition manifestement opposée à celle que la tige nous a présentée.

Moelle. Enfin, au centre de la racine se voil un tissu parenchymateux gorgé de sucs, qui représente la moelle; parfois, les faisceaux se joignant au centre, la moelle n'existe pas.

Accroissement de la racine en épaisseur.
Différences dans la structure de la racine des DicotyLÉdones, des Monocotylédones et des Acotylédones. - La structure qui vient d'être indiquée est exactement la même dans les trois embranchements du règne végétal, si l'on n’envisage la racine qu'à l'état jeune. Un peu plus tard apparaissent quelques différences.

Tandis que les choses restent en cet état chez un certain nombre de Dicotylédones, mais surtout chez les Monocolylédones et les Acotylédones (1), de sorte que leur's racines ne, s'épaississent pour ainsi dire pas, et qu'à ce point de vue il n'y a entre ces deux dernier's embranchements que des différences fort secondaires, il n'en est pas de même dans la plupart des Dicotylédones. Chez ces dernières, en effet, dans l'intervalle des faisceaux vasculaires primaires, il se

(1) Il ne s'agit, bien entendu, que des Cryptogames vasculaires, car seuls ils ont des vaisseaux, les Cryptogames cellulaires en étant totalement dépourvus. 
forme des faisceaux fibro-vasculaires secondaires, qui offrent les caractères de ceux de la tige, c'est-à-dire, qu'on y remarque, en allant de dedans en dehors, des vaisseaux, puis des fibres ligneuses, et enfin des fibres libériennes qui s'appliquent contre les faisceaux libériens primitifs. Ces faisceaux fibro-vasculaires présentent un accroissement continu, exactement comme ceux de la tige, de sorte que la racine augmente de grosseur chaque année. Telle est, dans la règle, la structure de la racine des Dicotylédones; les exceptions qu'elle présente ne doivent pas nous arrêter dans un ouvrage élémentaire.

L'utilité des vaisseaux et des fibres de la racine est considérable; comme on le verra plus loin, par les premiers les liquides que la racine puise dans le sol remontent jusque dans la tige; par les secondes, la sève élaborée redescend de la lige dans la racine jusqu'au point végétatif. 


\section{CHAPITRE V}

\section{Feuille.}

Définition et caractères des Feuilles : leur positıon sur la tige ; mode d'altache; mode de nervation; forme du limbe et ses modifications. - Structure des Feuilles : développement et croissance; épiderme et Stomates; Parenchyme foliaire; Nervures.

DÉfinition. - Les feuilles sont des organes appendiculaires, toujours portés par la tige, ordinaircment étalés en torme de lame, de couleur verte, et destinés à jouer un rôle considérable dans les fonctions vitales des plantes.

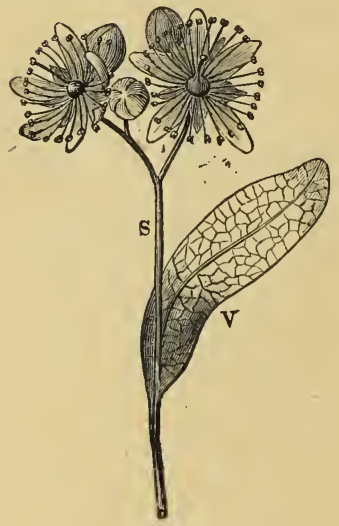

Fig. 71 .

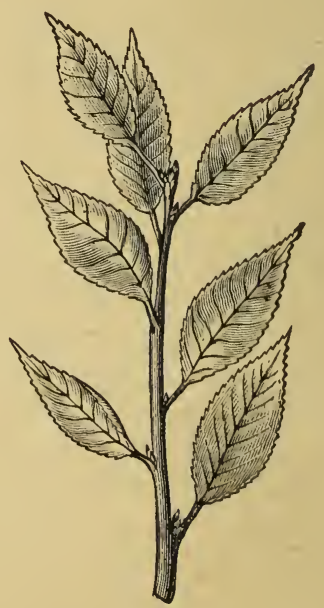

Fig. 72.

Fig. 71. - Fleurs de Tilleul $(s)$, avec une feuille transformće en bractée $(v)$. lig. 72. - Feuilles alternes (Prunus avium).

Caractères des feullles. - Une feuille se compose, en général, de deux parties, le pétiole et le limbe. 
Le pétiole ou pédoncule est le support de la feuille; il n'existe pas constamment; quand il manque, la feuille est dite sessile. Sa forme est variable : ordinairement arrondi, d'autres fois aplati, il prend dans quelques circonstances un grand développement; par exemple, lorsque le limbe

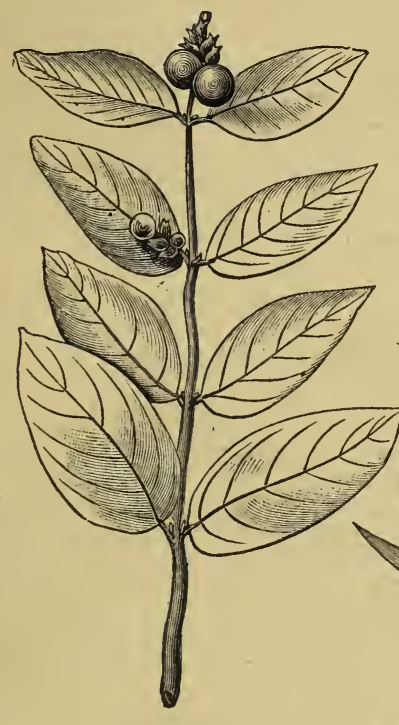

Fig. 73.

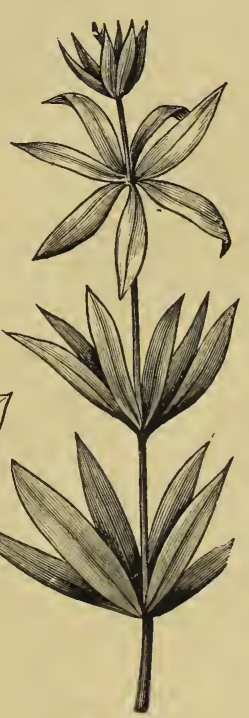

Fig. 71.

Fig. 73. - Feuilles opposées (Symphoricarpus racemosus). Fig. 74. - Feuilles verticillées (Asperula odorata).

fait défaut, il s'élargit et représente à lui seul toule la feuille, comme on le voit dans certains acacias, ce qui lui a

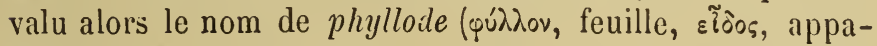
rence).

Le limbe ou partie étalée de la feuille offre les plus grandes variations de forme.

Sans entrer dans de grands détails sur les caractères présentés par les feuilles, on rappellera un certain nombre de définitions relatives à leur position sur la tige, à leur mode d'attache, aux nervures, aux particularités offertes par les bords du limbe, etc. 
Position des feuilles sur la tige.

On appelle feuilles caulinaires, celles qui naissent de la portion de tige visible au-dessus du sol; radicales, celles

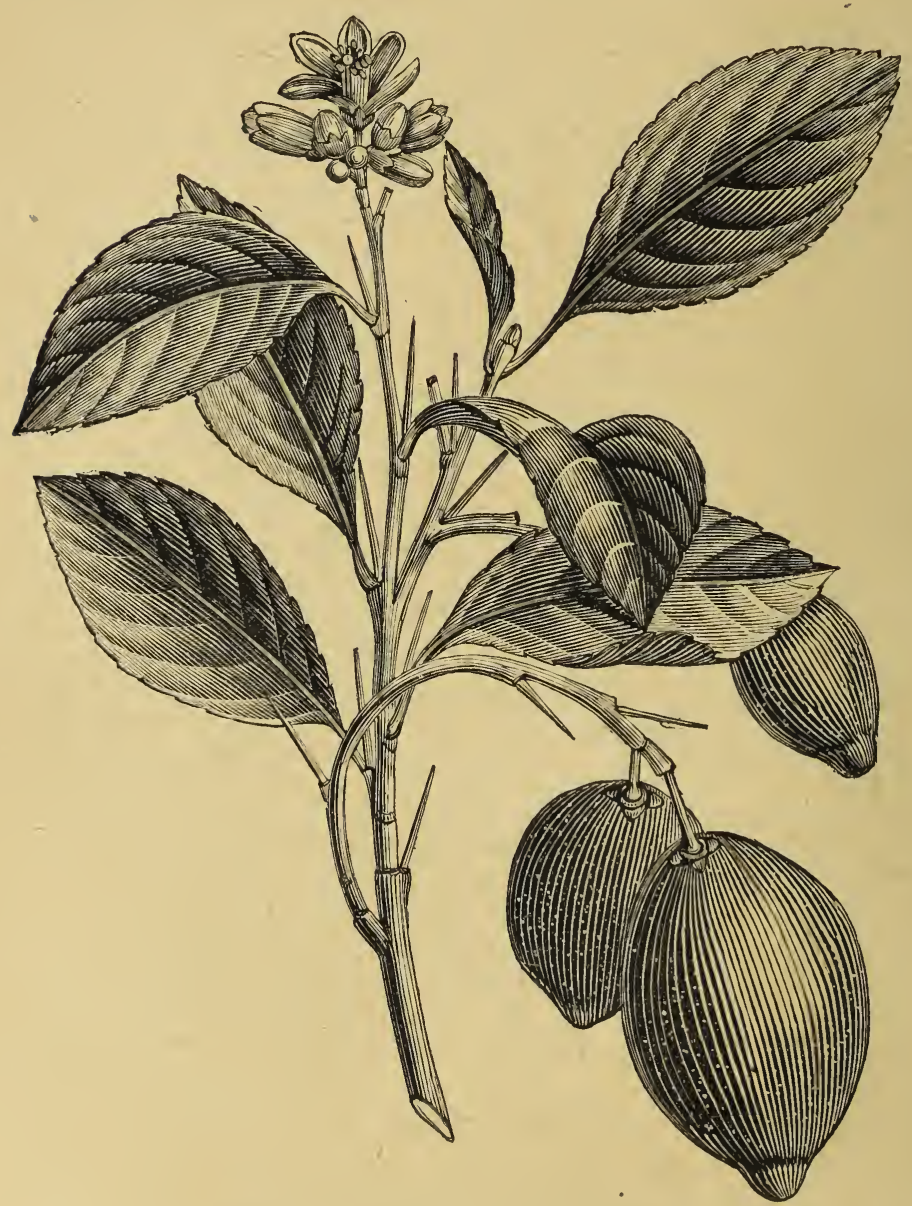

Fig. 75. - Feuilles articulées (Citronnier).

qui partent de la portion souterraine de la tige; florales, celles qui accompagnent les fleurs sans se modifier sensi- 
blement dans leur forme, par opposition aux braclées (fig. 71), qui sont des feuilles florales transformées en écailles.

Suivant les rapports de position qu'elles offrent entre elles sur la tige on les dit alternes ou opposées (fig. 72 et 73).

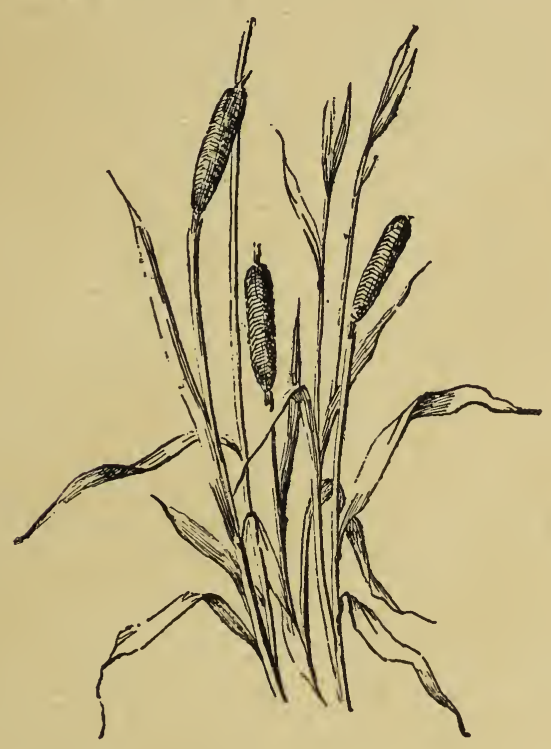

Fig. 76. - Feuilles embrassantes (Roscau).

Dans le premier cas, une seule feuille naît en un point donné; dans le second cas, deux feuilles naissent au même niveau, en deux points opposés de la tige. S'il y en a plus de deux ì partir du même niveau, trois, quatre, cinq, etc., les feuilles sont dites verticillees (fig. 74) (1).

Mode d'attache des feuilles.

On appelle feuille articulée (fig. 75), celle dont le pétiole présente à sa base un étranglement surmonté d'une sorte

(1) Cette question de la position relative des feuilles sur la tige ou phyllotaxie est traitée avec tout le développement qu'elle comporte dans le cours de la Classe de Cinquième. 
de bourrelet; embrassante ou amplexicaule(fig. 43 et 76), celle qui, avant de s'étaler en un limbe libre et flottant, enveloppe

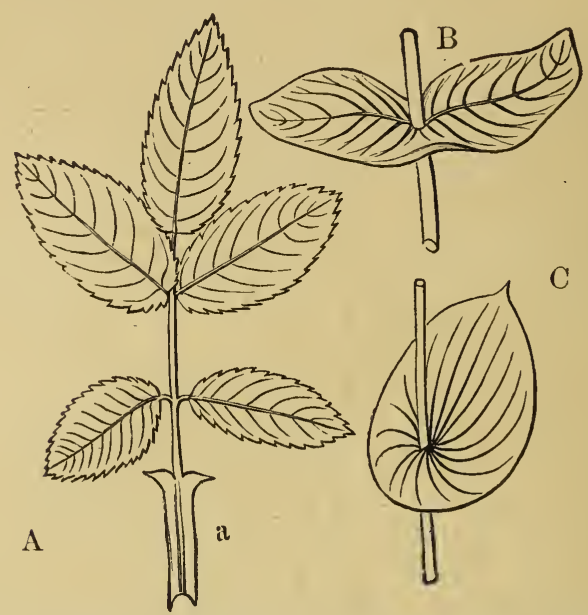

Fig. 77. - A, Feuille composée (Rosier) avec stipules $(a)$; B, Feuilles opposées soudées par leurs bases (Chèvre feuille); C, Feuille perfoliée (Buplèvre).

la tige d'une sorte de gaîne plus ou moinsallongée, cetle gaîne pouvant être fendue (Graminées), ou entière, c'est-à-dire à

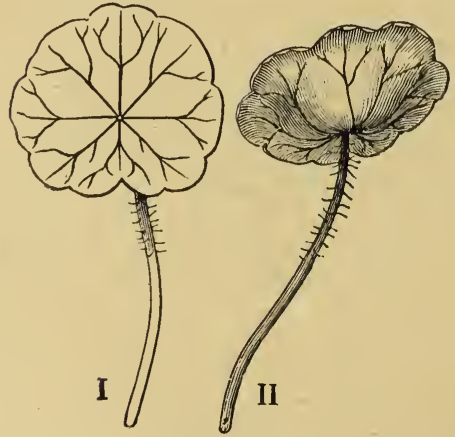

Fig. 78. - Feuille peltée (Hydrocotyle); $I$, vue en dessus; $I I$, vue en dessous. bords soudés(Carex); perfoliée, la feuille réduite à son limbe que semble traverser la tige (fig. 77, C); décurrente, celle dont les bords du limbe se prolongent en manière d'ailes le long de la tige.

Mode de nervation.

Les nervures de la feuille ne sont autre chose que les saillies linéaires produites par les faisceaux vasculaires émanés de la tige et qui ont traversé toute la longueur du 
pétiole; clles se voient surtout à la face inférieure du limbe. Il y a ordinairement dans les Dicotylédones une nervure ou côte médiane, plus grosse, puis des nervures secondaires (fig. 77, $A, B$ ) régulièrement disposées de part et d'autre de

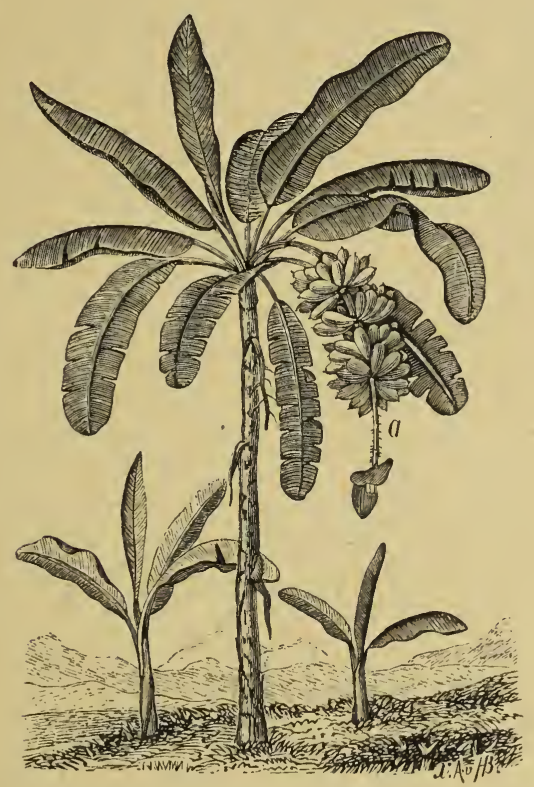

Fig. 79. - Feuilles rectinerviées de Monocotylédone (Bananier).

la précédente, des nervures tertiaires (fig. 33, 40, etc.), et la nervation est en réseau. Dans les Monocotylédones la disposition est plus simple : au lieu de se terminer par anastomoses comme les précédentes, les nervures sont, rectinerviées ou curvinerviées parallèles, entre elles, en long (fig. 43), ou en large (fig. 79); mais, dans ce dernier cas, à partir d'une côte médiane longitudinale, ou bien elles vont en divergeant à partir de la base du limbe.

Forme du limbe.

Les formes variées du limbe ont valu aux feuilles les noms d'ovales, arrondies ou orbiculaires, lancéolées, linéaires, subulèes, sagittées, réniformes, cordiformes, etc., qui s'expli- 
quent d'eux-mêmes; enfin, quand le limbe au lieu de se continuer par son bord avec le pétiole, lui donne insertion vers son milieu (fig. 78), la feuille est dite peltée (pelta, bouclier).

Particularités offertes par les bords du limbe.

La feuille est entière quand le bord du limbe est uni, sans dentelures; dentée, serrée (serra, scie), quand il est découpé en forme de dents un peu recourbées comme celles d'une scie; crénelée, quand les découpures sont larges et obtuses.

Lorsque le limbe est partagé, par des incisions, en lobes distincts, on dit la feuille bifide, trifide, quadrifide, quinquéfide ou multifide, bilobée, trilobée, etc., bipartite, tripartite, etc., ou pinnatiséquée, suivant que les divisions sont peu profondes, vont un peu plus avant, ou atteignentenfin presque la base du limbe. Si les incisions sont irrégulières, la feuille est dite laciniée, et pinnatifide quand les incisions sont profondes et les lobes disposés régulièrement à la facon des folioles d'une feuille composée-pennée; enfin, palmée, quand elles divisent le limbe en lobes réguliers disposés en rayonnant à partir du solnmet du pétiole (Ricin, Vigne, fig. 41). T'outes ces feuilles sont dites simples.

Un degré de plus dans la disposition précédente caractérise les feuilles composées, c'est-à-dire, celles qui sont formées par des lobes ou folioles nellement séparés et distants les uns des autres, tous portés par un pétiole commun ou par autant de pétioles secondaires insérés sur un pétiole primaire (fig. $77, \mathrm{~A}$ ). Les principales dispositions qu'elles affectent leur valent les noms de feuilles penriées (fig. 37), digitées (fig. 77, A), surdécomposées, les divisions primaires en portant de secondaires. Chacune de ces formes offre plusieurs variétés.

STRUGTURE DE LA FEUILLE

Développeyent et Croissance des feullles. - Le bourgeon, dont il a été déjå question à propos de l'accroissement 
de la tige en hauteur, p. 70, doit être également étudié avec soin si l'on veut se rendre compte du mode de développement des feuilles. Ce sont ces dernières qui, en somme, constituent presque toute sa masse en formant une épaisse enveloppe autour du sommet végétatif de la tige (fig. 5̌4).

Chaque feuille apparaît sous forme d'un petit mamelon arrondi, dû à une excroissance que produit la tige près de son sommet, et qui en s'allongeant, se recourbe au-dessus du point végétatif. Il est d'une structure entièrement homogène, formé de cellules toutes semblables, et dont celles qui occupent le sommet se divisent, se multiplient pour allonger la jeune feuille. Mais le développement de la feuille par le sommet ou, comme on dit, par croissance terminale, s'arrête bientôt pour faire place, ainsi qu'on va le voir, à la croissance intercalaire.

G'est toujours le limbe qui apparaît le premier, le pétiole ne se développant que plus tard. Or, la croissance du limbe peut se faire à partir d'une zone qui tantôt est placée très près de la base, et alors les parties apparaissant successivement du sommet à la base, la croissance est basipète, et tantôt placée près du sommet, les parties se développant dans ce cas de la base au sommet, et la croissance est basifuge. Enfin cette zone peut occuper une région intermédiaire, et la croissance ayant lieu tant du côté de la base que du côté du sommet, elle est dite mixte.

D'autre part, il peut arriver que le limbe se développe par tous ses points à la fois; la croissance est alors non plus successive, comme dans les cas précédents, mais simultanée.

Structure de la feuille entièrement développée. Épiderme et Stomates. - Dans la plupart des cas, la feuille se compose d'un épiderme supérieur et d'un épiderme inférieur, entre lesquels se trouve compris un tissu parenchymateux et des faisceaux fibro-vasculaires que l'on voit faire saillie à l'extérieur, sous forme de nervures.

L'épiderme n'est pas absolument identique à la face supérieure et à la face inférieure du limbe de la feuille. 
En général, il ne comprend qu'une assise de cellules; mais dans certaines feuilles, celles qui sont coriaces, par exemple, il peut en offrir deux ou trois. La forme de ces éléments est très variable, non seulement sur des végétaux différents, mais sur une même plante, d'une feuille à l'autre, bien plus, sur deux portions voisines de la même feuille. Presque toujours aplaties, tabulaires, elles sont rectangulaires, allongées, et régulièrement alignées, ou bien à bords sinueux et s'engrénant avec ceux des cellules voisines. Le plus souvent incolores, elles offrent quelquefois de la chlorophylle, par exemple, chez les Fougères.

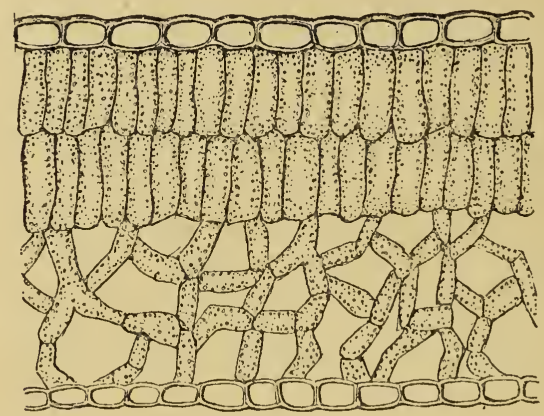

Fig. 80. - Portion d'une coupe transversale de feuille. Entre les assises supérieure et inférieure des cellules épidermiques, on voit des cellules allongées sur deux rangs (parenchyme en palissade), au-ilessous desquelles sont des cellules de forme irrégulière (parenchyme rameux), avec nombreux méats intercellulaires.

La surface de l'épiderme se cuticularise le plus souvent, c'est-à-dire, se recouvre d'une cuticule ; en outre on y voit fréquemment des poils, surtout ì la face inférieure de la feuille.

Stomates.

Une des particularités les plus importantes de la structure

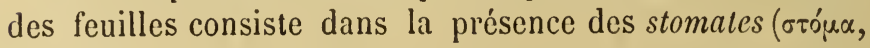
bouche), petites ouvertures par le moyen desquelles l'air almosphérique peut communiquer avec le parenchyme interne. Le plus souvent, l'épiderme supérieur en est complètement dépourvu ou n'en possède qu'un petit nombre, 
tandis que l'inférieur en offre une grande quantité. Ils sont parfois si rapprochés, qu'un millimètre carré en renferme plusieurs centaines. Rangés en lignes parallèles sur les feuilles des Monocotylédones, ils sont disposés sans ordre sur celles des Dicotylédones.

Dans les plantes aquatiques dont les feuilles flottent à la surface de l'eau, c'est au contraire la face supérieure, seule en contact avec l'atmosphère, qui en présente. Enfin dans les plantes complètement submergées on n'en trouve ni sur une face, ni sur l'autre, bien que cette règle comporte quelques exceptions.

Un stomate se compose de deux petites cellules qui se regardent par un bord concave, de façon à limiter entre

Structure des Stomates.

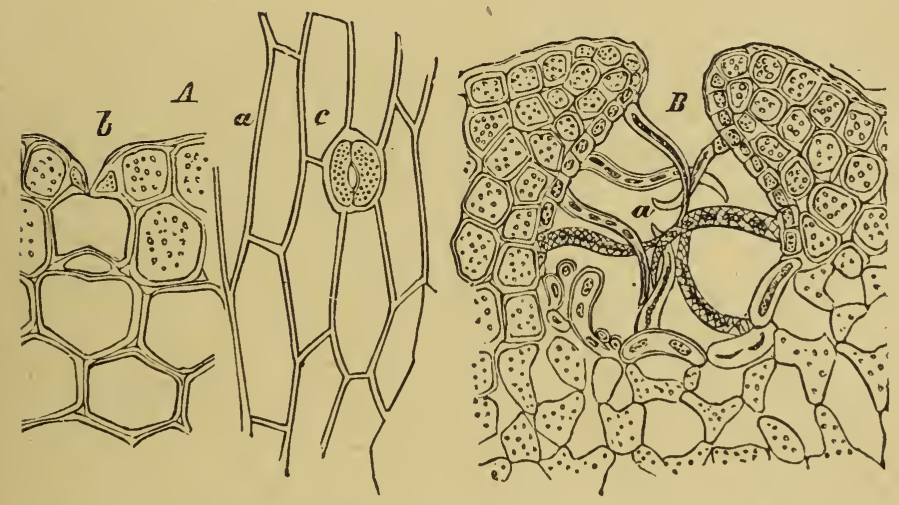

Fig. 81. - Stomates; $\boldsymbol{A}$, stomates de la Jacinthe; $a$, lambeau d'épiderme de la feuille vu de face; $c$, stomate; $b$, coupc transversale de la même feuille passant par un stomatc. $B$, coupe transversale d'une feuille de Laurier-rose passant par une cavité ou crypte dont le fond offre des stomates et dont les parois sont hérissécs de poils $(a)$.

elles un étroit orifice, qui a reçu le nom d'ostiole (fig. 81, $A, c)$. Parfois les stomates sont logés dans des cavités, des enfoncements creusés dans les couches superficielles de la feuille, disposition dont le Laurier-rose offre un exemple des plus remarquables $(\boldsymbol{B})$. Ces petites chambres peuvent renfermer un seul ou plusieurs stomates. Au-dessous de 
chaque stomate se trouve un petit espace résultant de l'écartement des cellules sous-jacentes ct qu'on appelle chambre aérienne ou sous-stomatique. Dans cette chambre débouchent, d'autre part, les méats dont le parenchyme est creusé.

Parenchyme foliaire. - C'est lui qui forme la plus grande partie du tissu de la feuille; son aspect n'est pas partout le même. Du côté de l'épiderme supérieur, il se compose de cellules allongées et serrées les unes près des autres, sans laisser d'interstices, à la manière de pieux. d'où le nom de parenchyme en palissade, qu'on lui donne souvent (fig. 80). Du côté de l'épiderme inférieur, il est formé de cellules fort irrégulières offrant des prolongements en divers sens et limitant des espaces lacuneux ou méats inlercellulaires dans lesquels circulent les gaz. Toutes ces cellules renferment de la matière verte ou chlorophylle, mais en plus grande abondance dans les cellules en palissade, ce qui, joint à ce que le tissu est plus serré dans cette région, donne à la feuille une coulcur d'un vert plus foncé sur cette face que sur l'autre. C'est dans le parenchyme que se passent les modifications éprouvées par la chlorophylle, qui font passer les feuilles de la couleur verte à ces belles teintes rouges et jaunes qu'elles présentent si souvent à l'automne.

Le parenchyme acquiert son maximum de développement dans les plantes grasses, ainsi appelées en raison de l'épaisseur de leurs feuilles molles et aqueuses, on même temps qu'elles sont peu riches en chlorophylle.

Nervures des feuilles. - Le parenchyme foliaire est traversé, sillonné par les nervures, qui vont en s'alténuant de la base de la feuille à la périphérie ct qui dessinent à sa surface des réseaux fort compliqués chez les Dicotylédones, ou bien des lignes parallèles toutes de grosseur sensiblement égale, chez les Monocotylédones. Dans les Fougères, leur disposition est assez variée pour que l'on puisse fonder sur elle de bons caractères pour la classification des genres et des espèces. 
Les nervures ne sont autre chose que des faisceaux fibrovasculaires venus de la tige à travers l'écorce et passant par le pétiole de la feuille, qui en est à peu près entièrement formé, pour s’épanouir et se ramifier dans le limbe. On y trouve, par conséquent, les mêmes éléments que nous avons vus dans la tige, à savoir, des fibres libériennes, des fibres ligneuses et des vaisseaux, avec une zone génératrice très étroite et dont l'activité est promptement limitée. A mesure que les nervures deviennent plus grêles, les fibres et les vaisseaux disparaissent successivement, les trachées persistant les dernières. 


\section{GHAPITRE VI}

\section{FONCTIONS DE NUTRITION}

\section{Absorption; Alimentation; Circulation ; Transpiration.}

Division des fonctions de nutrition. - ABsorptron. Différentes voles d'absorption : $1^{\circ}$ absorption par les racines; $2^{\circ}$ par les feuilles; $3^{\circ}$ par quelques autres parties. - AlimeNts des véGÉTAux. Ce qu'on doit entendre par aliments. Origine des principaux aliments des végétaux. - Circulation. Ce qu'il faut entendre par circulation chez les plantes. Ascension de la sève : force d'ascension de la sève; causes de la montée de la sève; composition de la sève ascendante; voies suivies par la sève ascendante. Sève descendante; voies suivies par la sève descendante. Circulation du protoplasma et du latex. - Transpiration. Nature de cette fonction. Siège de la transpiration. Activité de la transpiration. Conditions qui modifient cette fonction.

Division des fonctions de nutrition. - ' Les fonctions de nutrition sont fort compliquées chez la Plante et comparables, comme l'a surtout montré Claude Bernard, à celles de l'Animal.

Nous aurons à étudier, en effet, dans un premier chapitre, l'Absorption, les Aliments des végétaux, la Circulation, la Transpiration, et dans le chapitre suivant, la Respiration et la Fonction chlorophyllienne, la Digestion, l'Assimilation.

Absorption.

Différentes voies d'absorption. - Les plantes possèdent deux voies principales d'absorption, à savoir leurs 
racines et leurs feuilles. Mais elles peuvent encore absorber par d'autres organes, tels que les fleurs, les graines, etc., et aussi par certaines parties de la tige, d'où la division naturelle de ce chapitre en trois sections principales.

$1^{0}$ Absorption par les racines. - Les racines absorbent par leur extrémité, non pas toutefois par leur pointe même, comme on l'a cru longtemps, car celle-ci est revêtue de la pilorhize, formée de couches cellulaires inertes, mais par la portion située immédiatement au-dessus et qui présente les poils cellulaires, auxquels on a donné le nom de sucoirs (fig. 69). Ces poils radicaux sont les principaux organes de l'absorption; mais celle-ci se fait encore par les parties de la racine recouvertes d'un mince épiderme.

Les substances absorbées sont de deux ordres, des liquides et des gaz, tous les physiologistes étant unanimes Nature des substances à reconnaître que les solides, quelle que soit leur tenuité, ne sauraient être absorbés s'ils ne sont préalablement dissous.

De l'examen des observations dues à de Saussure, Liebig, Ce que l'on sait Bouchardat, Sachs, Duchartre, Baillon, etc., relativement de l'absorption. à l'absorption par les racines, plusieurs points restent acquis; il importe de les noter: 1o si l'eau dans laquelle plonge une racine tient une substance en dissolution, l'eau sera absorbée en plus grande proportion que la substance dissoute, de sorte que la solution aura tendance à se concentrer de plus en plus; $2^{\circ}$ de plusieurs substances en dissolution dans l'eau, celles-là seront absorbées en plus grande proportion qui sont à un état de fluidité plus grande ; $3^{\circ}$ la racine n'a pas la faculté de choisir telle substance soluble à l'exclusion de telle autre égale. ment soluble, celle-ci fût-elle même un poison pour la plante, laquelle n'a pas, en somme, la faculté d'élection; $4^{\circ}$ les plantes peuvent puiser dans le sol des substances insolubles dans l'eau, comme du calcaire, grâce à la propriété qu'elles ont de sécréter des substances acides, notamment de l'acide carbonique, qui rend les premières solubles, comme le prouvent les observations de Becquerel el les 
expériences de Liebig. C'est ainsi, encore, que des débris de corne se consomment plus rapidement dans un terrain où existe une riche végétation, que dans un terrain dépourvu de plantes.

Les substances colorantes en dissolution sont-elles absorbables?

Mais on est peu d'accord pour décider si les substances colorantes à l'état de dissolution sont susceptibles d'être absorbées. Ainsi, en plaçant un oignon de Jacinthe à fleurs blanches dans un vase plein d'eau colorée en rouge par le suc d'une plante, le Phytolacca decandra, on peut observer que les pétales des fleurs se colorent de traînées rouges, évidemment dues au liquide absorbé. Mais on a objecté à ce résultat qui, au premier abord, paraît si concluant, que le plateau de l'oignon qui a été mis au contact de l'eau colorée, est un tissu cicatriciel qui offre des solutions de continuité, de petites déchirures, par lesquelles le liquide pénètre dans la plante, et que si l'on a soin que les racines seules soient immergées dans le liquide, la coloration des pétales ne se produit pas.

Il faudrait donc admettre, d'après cela, contrairement à ce qui a été dit plus haut, que les racines font un certain choix même parmi les substances dissoutes; aussi plusieurs botanistes sont-ils portés à croire que dans le cas d'absorption de substances nuisibles, de poisons, il y a d'abord une altération des parties de la racine en contact avec elles, à la suite de quoi ces dernières pénètrent dans la plante par une sorte d'imbibition toute p ysique.

$2^{\circ}$ Absorption par les feuilles. - $1^{\circ}$ Absorption des $g a \%$. Il est hors de doute que les feuilles absorbent activement les fluides aériformes de l'atmosphère, tels que l'oxygène, l'acide carbonique et même des gaz ou des vapeurs nuisibles, comme les vapeurs mercurielles, qui ont d'ailleurs pour résultat de tuer les éléments cellulaires. Cette absorption ne se fait pas indifféremment par toutes les parties de la feuille; c'est seulement là où existent des stomates que la fonction s'accomplit. Toutefois, elle peut aussi avoir lieu à travers les parois 
très délicates de certains organes, celles des pétales des fleurs, par exemple.

$2^{\circ}$ Absorption des liquides et de la vapeur d'eau. Ici il existe une divergence d'opinion entre les expérimentateurs. Ainsi M. Duchartre met au contact de l'eau les feuilles d'une plante venue en pot; il la pèse avant et après l'expérience et il n'observe pas d'augmentation de poids; il en conclut que les feuilles n'absorbent pas l'eau à l'état liquide. D'autres expérimentateurs, M. Baillon, entre autres, combattent cette conclusion par quelques expériences bien simples. Les feuilles d'une plante aquatique, le Potamogeton, se desséchent et se fanent rapidement quand elle est retirée de l'eau, mais quand on les y replonge, ces mêmes feuilles reprennent très vite leur aspect primitif.

Une autre expérience due à Hales tend à confirmer l'absorption de l'eau par les feuilles. Il immerge une portion de branche portant deux rameaux feuillus, de facon que l'un de ceux-ci reste en dehors de l'eau; on peut alors remarquer que, non seulement, les feuilles immergées restent fraîches, mais aussi celles qui ne sont pas en contact avec l'eau, dans lesquelles le liquide que les premières ont absorbé est arrivé par l'intermédiaire de la tige. Dans cette expérience on a soin, bien entendu, de tenir en dehors de l'eau la surface de section de la tige.

Il faut bien remarquer d'ailleurs que l'absorption ne s'exerce pas par toutes les parties des feuilles; en effet, les endroits revêtus d'une matière cireuse ou de poils serrés qui retiennent une couche d'air, ne peuvent pas être mouillés; l'eau ne peut y adhérer, et l'absorption ne s'y fait pas. En plongeant une feuille dans l'eau, on reconnaît facilement, à première vue, les parties qui se mouillent à l'exclusion des autres, et par là même celles qui sont susceptibles d'absorber et celles qui ne le sont pas. On pourra aussi remarquer que la face de la feuille où les stomates sont le plus nombreux, est celle que l'eau a le plus de peine à mouiller.

La même divergence existe entre les observateurs au 
sujet de l'absorption de l'eau à l'état de vapeur. Tandis que les uns dénient aux feuilles la possibilité de l'absorber, les autres la lui accordent.

Faisons remarquer, en terminant cette importante question de physiologie, que l'une des causes de la différence des résultats obtenus consiste en ce que les expériences n'ont pas été faites dans des conditions identiques. S'il s'agit, en effet, de plantes gorgées de suc et n'ayant pas de pertes à réparer, il n'y a pas absorption par les feuilles; tandis que si l'on expérimente sur une plante flétrie par suite de l'épuisement de l'eau du sol dans lequel elle végète, les feuilles absorbent l'eau mise à leur contact, soit à l'état liquide soit à l'état de vapeur.

$3^{\circ}$ Absorption par quelques autres parties de la plante. - Sans nous arrêter aux phénomènes d'absorption dont certaines parties des plantes recouvertes d'un mince épiderme sont le siège, telles que les portions jeunes de la tige, les pétales des fleurs, etc., nous dirons quelques mots de l'absorption des liquides par le pollen et les graines.

Les grains de pollen absorbent très facilement les liquides au contact desquels ils sont mis; c'est grâce à cette propriété que ces petits corps peuvent germer, c'est-à-dire, produire le tube ou boyau pollinique qui s'enfonce à travers lc stigmate et le style du carpelle, pour pénétrer jusqu'aux ovules. Leur faculté d'absorption est même pousséc au point que, si le liquide est abondant ou trop aqueux, le tube pollinique éclate, son contenu se répand et la fécondation des ovules ne peut s'accomplir, c'est ainsi que la coulure est déterminée par les grandes pluies qui surviennent au moment de la pollinisation. (Voy. Chap. x.)

Enfin, on n'ignore pas que les graines au moment de la germination absorbent l'eau avec une assez grande activité, de façon à se gonfler énormément et à faire éclater les enveloppes qui les recouvrent, pour permettre à la jeune plante de se faire jour au dehors. 
Aliments des Végétaux.

LES ALIMENTS DES VÉGÉTAUX SE RAMÈNENT A QUELQUES SUBSTANCES CHIMIQUES. - L'absorption ne pouvant s'exercer que sur des substances gazeuses, liquides ou des corps solides en dissolution, les aliments des plantes doivent se présenter, pour être absorbés, sous l'un de ces ètats.

L'étude des substances qu'on trouve toutes formées dans les végétaux, lesquelles font partie de leur constitution et qu'on appelle, en un mot, leurs principes immédiats, est fort complexe, car il en existe une très grande variété; mais toutes, en somme, résultent de la combinaison d'un nomb re très restreint d'éléments chimiques auxquels se ramènent tous les aliments du végétal, et qui vont seuls nous occuper pour le moment.

CE QU'ON doit entendre par alinent. - Et d'abord, doiton considérer comme aliment toute substance qui a pénétré dans l'organisme végétal ? Assurément non. Gertains corps sy trouvent, en effet, en proportion tellement minime que leur présence peut être négligée et considérée comme accidentelle. En outre, toute substance introduite dans l'organisme végétal, même en assez notable quantité, n'est pas nécessairement un aliment; autrement on serait amené à considérer comme tel même des substances toxiques, puisque nous avons vu plus haut que la plante pouvait en absorber.

En somme, on ne doit regarder comme aliments que les substances qui concourent réellement à la nutrition de la plante.

Ces restrictions faites, les aliments ou plutôt les substances chimiques qui composent les aliments des végétaux sont le Carbone, l'Oxygène, l'Azote, l'Hydrogène, le Soufre, le Phosphore, le Calcium, le Potassium, le Magnésium, le 
Fer, auxquels on peut encore ajouter le Chlore, le Sodium, le Brome, l'Iode, etc. En somme, on trouve surtout six corps simples non métalliques, quatre métalliques, et quelques autres secondaires ou accidentels.

C'est de la combinaison de ce petit nombre d'éléments introduits dans l'organisation végétale que résultent tous les tissus et tous les produits végétaux, la cellulose, l'amidon, le sucre, les matières albuminoïdes, les alcaloïdes, les matières grasses, cireuses, les gommes, les sels, etc.

Source des principaux aliments des végétaux. - Il ne sera pas sans intérêt de rechercher l'origine des corps élémentaires des végétaux, en nous bornant aux principaux d'entre eux.

$1^{\circ}$ Carbone. C'est l'un des éléments les plus importants ; il se rencontre dans toutes les plantes, en grande proportion, aussi bien dans la paroi même des cellules et des vaisseaux, que dans les substances qu'clles renferment. Il vient de deux sources, à savoir, de l'air et du sol. L'air renferme constamment de l'acide carbonique, en très faible proportion, il est vrai, de 4 à 6 dix-millièmes; mais si l'on songe à l'étendue de l'atmosphère qui enveloppe toute la terre, on verra, comme le calcul approximatif en a été fait, que c'est à des centaines de milliards de kilogrammes qu'il faut évaluer la masse de carbone en suspension dans l'atmosphère. Ajoutons que, malgré la quantité que les plantes en prennent journellement par leurs feuilles, la masse totale ne saurait diminuer, car la destruction incessante des organismes animaux et végétaux, la respiration de tous les êtres vivants, les combustions de toute sorte ont pour effet de restituer à l'atmosphère le carbone qui lui est enlevé. D'autre part, les courants atmosphériques ont pour résultat de répartirégalement la proportion d'acide carbonique qui, sans cela, pourrait se trouver en voie de diminution dans les régions où existe une riche végétation.

L'autre source du carbone, c'est le sol ; des expériences montrent, en effet, que l'absorption du carbone par les feuilles ne rend pas suffisamment compte de l'augmenta- 
tion de la quantité de carbone acquise pendant une période végétative déterminée. Bien plus, les Champignons, et tous les végétaux sans chlorophylle, sont incapables de prendre le carbone de l'air; c'est à la terre seule qu'ils empruntent celui qui entre dans leur constitution. Toutes les plantes puisent également dans le sol, par leurs racines, une certaine quantité de carbone à l'état d'acide carbonique ou de carbonate en solution dans l'eau.

$2^{\circ}$ Oxygène. Il est pris, d'une part, directement à l'atmosphère dans le phénomène de la respiration, et aussi à l'état de combinaison avec le carbone (acide carbonique); une partie de l'oxygène de ce dernier est aussitôt rejetée, il est vrai, mais il en reste encore dans la plante 1/3 environ, d'après Sénebier et de Saussure. D'autre part, on comprend que l'eau pompée dans le sol par les racines fournit une autre proportion importante de l'oxygène nécessaire à la plante.

$3^{\circ}$ Hydrogène. Il provient également de l'eau puisée par les racines, et aussi de la décomposition des substances ammoniacales qui sont si abondantes dans les débris organiques végétaux et animaux.

$4^{\circ}$ Azole. Très répandu dans les végétaux, quoique en bien moindre proportion que chez les animaux, l'azote entre dans la constitution d'un grand nombre de principes immédiats. Il provient des matières ammoniacales et principalement des nitrates de potasse et de soude répandus à la surface du sol, contenus dans les engrais, etc.

M. Boussingault avait conclu de quelques expériences que l'azote est en partie puisé dans l'atmosphère, et même il avait cru pouvoir montrer que certaines plantes lui empruntent à peu près exclusivement leur azote, tandis que d'autres l'enlèvent au sol, de sorte que les premières auraient pour effet, au point de vue de la culture, d'améliorer la terre, comme seraient le Trèfle, les Légumineuses, tandis que les autres auraient pour résultat de l'épuiser, les Céréales, par exemple. Mais des recherches ultérieures lui prouvèrent que ses premières conclusions étaient erronées et qu'en réalité, l'azote de l'air ne joue aucun rôle dans la végétation, ou un rôle si minime qu'on doit le négliger. 
こ० Soufre. La plupart des matières albuminoïdes contiennent une certaine proportion de soufre; bien plus, certains produits végétaux en renferment une quantité considérable (huile essentielle d'Ail, de Moutarde). Il est fourni aux plantes par les sulfates solubles contenus dans le sol.

$6^{0}$ Phosphore. On le rencontre à l'état de combinaison dans un certain nombre de substances organiques végétales; il paraît provenir des phosphates du sol, tels que ccux de potasse, de soude, de chaux, de magnésic.

Autres principes alimentaires des végétaux. Parmi les cendres, résidu de la combustion des végétaux, on trouve un grand nombre de principes minéraux et salins dont il nous faut dire quelques mots. Parmi eux il en est d'indispensables à la plante, et d'autres tout à fait accessoires. Les premiers sont, comme il a été dit plus haut, le potassium, le calcium, le magnésium, le fer. Sans eux la plante paraît ne pas pouvoir se développer ; ainsi, l'on a observé que des graines de Sarrasin ou Blé noir en germination, auxquelles on fournit tous les éléments nécessaires à leur végétation, à l'exception du potassium, ne croissent pas et meurent en peu de temps. De même, une plante qui est absolument privée de fer, devient chlorotique; ses feuilles pâlissent et prennent une coloration blanche, puis la plante elle-même finit par mourir d'inanition; mais si on l'arrose d'une solution ferrugineuse, la santé lui revient avec sa couleur normale.

Quant aux autres principes qui se rencontrent dans les cendres des végétaux, ils ne semblent pas indispensables, bien qu'on ne puisse douter que plusieurs, tout au moins, soient de quelque utilité dans l'économie de la plante. Ainsi, le sodium se rencontre dans un très grand nombre d'espèces; cependant la plupart des physiologistes ne le considèrent que comme étant d'une très faible utilité. Le chlore, le brôme, l'iode ont une action assez mal définic, mais qui semble, tout au moins pour les espèces marines, n'être pas douteuse. La silice, qui est si généralement répandue dans les plantes, et parfois en proportion considérable, par exemple, dans les Graminées, les Équisélacées, 
ne paraît cependant pas indispensable à leur végétation, comme le prouve la germination el le développement sur un sol complètement dépourvu de silice, de végétaux qui en possèdent d'or'dinaire.

Remarques. - $1^{0}$ Toutes les substances qui viennent d'être passées en revue ne méritent pas, d'une façon rigoureuse, le nom d'aliments ; en effet, elles ne sont pas assimilées telles quelles par la plante et employées de suite dans la nutrition, mais constituent les éléments au moyen desquels les aliments proprement dits sont fabriqués, élaborés par elle. Ce résultat final est précédé d'une série d'ópérations organiques dont le siège principal se trouve dans les feuilles, grâce auxquelles les minéraux empruntés au sol ou à l'atmosphère entrent dans des combinaisons nouvelles et sont l'endus aptes à être ensuite assimilés, afin d'accroître le végétal et d'en renouveler les parties usées et hors de service.

$2^{\circ}$ Ajoutons que chez les plantes, comme chez les animaux, on peut, d'une façon générale, adopter une classification physiologique des aliments qui, sans être d'une exactitude très rigoureuse, est commode pour l'élude, à savoir, les aliments plastiques, qui servent surtout à l'entretien et à la formation des tissus, les aliments respiratoires, destinés surtout à être brûlés, consumés dans l'organisme végétal et qui s'y trouvent souvent accumulés à l'état de combinaisons ternaires, comme l'amidon, le sucre, etc.

$3^{\circ}$ Enfin, on peut se demander à quel règne appartiennent les aliments puisés dans le sol par les racines, autrement dit, s'ils sont à l'état de matière organique ou de matière inorganique. Liebig a vivement soutenu l'opinion que la Plante ne se nourrissait que de composés inorganiques, d'où le précepte de ne lui fournir que des engrais exclusivement minéraux. Cette manière de voir est trop absolue, bien qu'il soit parfaitement constaté que le rôle général des végétaux dans la nature soit de transformer les substances inorganiques en matières organiques et de préparer par là même la nourriture des animaux. Une expérience bien des fois séculaire a montré, en effet, que les engrais organiques, les 
fumiers, activent notablement la végétation, et qu'ils sont même préférables aux engrais minéraux.

Circulation.

Ce qu'il faut entendre par Girculation chez la plante. - Dans la Plante, comme dans l'Animal, un liquide nutritif circule et entretient la vie dans toutes les parties de l'organisme; au lieu d'être du sang, c'est de la sève. Toutefois, hâtons-nous d'ajouter que la circulation de la sève n'est pas identique à la circulation du sang. Le but est le même, mais les moyens employés pour l'atteindre sont fort différents; il faudrait donc bien se garder d'assimiler les deux phénomènes.

L'étude de la circulation de la sève se partage naturellement en deux parties, selon qu'il s'agit du mouvement de la sève brute, c'est-à-dire, des liquides puisés dans le sol par les racines et qui, de celles-ci, va se répandre dans toute la plante, ou bien de la sève élaborée, qui suit une marche inverse, redescendant des parties supérieures de la plante vers les racines.

Ascension de la sève. - Les aliments puisés dans le sol et tenus en dissolution dans l'eau doivent être distribués dans toutes les parties du végétal, pour que chaque élément cellulaire y puise ce qui lui est utile. Mais avant que le travail d'assimilation s'accomplisse, il est indispensable que ces principes subissent des moditications chimiques, se combinent entre eux de façons différentes sous l'influence vitale des cellules. Le siège de ce travail paraît être surtout dans les feuilles; c'est donc vers elles que le liquide puisé par les racines va tout d'abord se diriger.

$\mathrm{Si}$, au retour des beaux jours, quand la tiède chaleur du printemps commence à se faire sentir, on coupe une 
branche, qui naguère semblait desséchée, un rameau de Vigne, par exemple, on voit sourdre à la surface de section des gouttes d'un liquide très aqueux, qu'on appelle vulgaire ment les pleurs de la Vigne. Ce liquide n'est autre chose que la sève, c'est-à-dire, les sucs puisés dans le sol, qui gagnent de proche en proche les parties les plus élevées de la tige. Son mouvement d'ascension se continue la nuit aussi bien que le jour et il est assez rapide, un mètre environ en une heure et demie.

Force d'ascension de la sève. - Un physiologiste anglais, Hales, a calculé la force avec laquelle l'eau du sol est ainsi aspirée par les racines, de façon à la faire monter jusque dans les feuilles, si élevées qu'elles soient. Pour cela, il appliqua à la surface de section d'un rameau de Vigne un manomètre formé d'un tube recourbé en $\mathrm{S}$, dans la plus grande branche verticale duquel il versa du mercure qui vint remplir la courbure inférieure. Il vit alors que la sève qui s'écoulait du rameau coupé, en pénétrant dans le manomètre, refoulait le mercure jusqu'à une hauteur de trois pieds. Si l'on suppose le mercure remplacé par de l'eau, on voit que la poussée de la sève ferait équilibre à une colonne d'eau de près de 13 mètres. Cette même expérience faite sur le Bouleau donne un résultat presque double, soit 30 mètres environ. La figure 82 représente cette expérience : une jeune tige $(a)$, élevée en pot, est coupée à quelques centimètres au-dessus du sol et sa surface de section engagée à travers un bouchon de caoutchouc, jusque dans l'inlérieur d'un tube de verre, dont la partie supérieure rétrécie est pourvue d'un robinet $(c)$ et se termine par un entonnoir. Ce tube communique par le côté avec un manomètre à mercure $(d, f, e, n)$, maintenu en place par une pince $(g)$. Le robinet étant ouvert, on verse de l'eau par l'entonnoir, jusqu'à remplir l'appareil; puis on verse du mrercue dans le tube manométrique de façon qu'il arrive dans le réservoir jusqu'au niveau de la ligne $e n$, en refoulant l'excédant de l'eau, quise déverse au dehors par le robinet. Ce dernier est alors fermé et l'appareil abandonné à lui-même. De la 
surfice de section (a) la sève s'écoulc bicntôt et pousse le mercure, qui monte dans le tube manométrique. Bien entendu, la terre qui entoure les racines doit être tenue humide. La hautcur à laquelle arrive le mercure est variable suivant les espèces. Pour beaucoup de plantes cllc attcint une demi-atmosphère, soit $380^{\mathrm{mm}}$, , pour d'autres $760^{\mathrm{mm}}$. et plus.

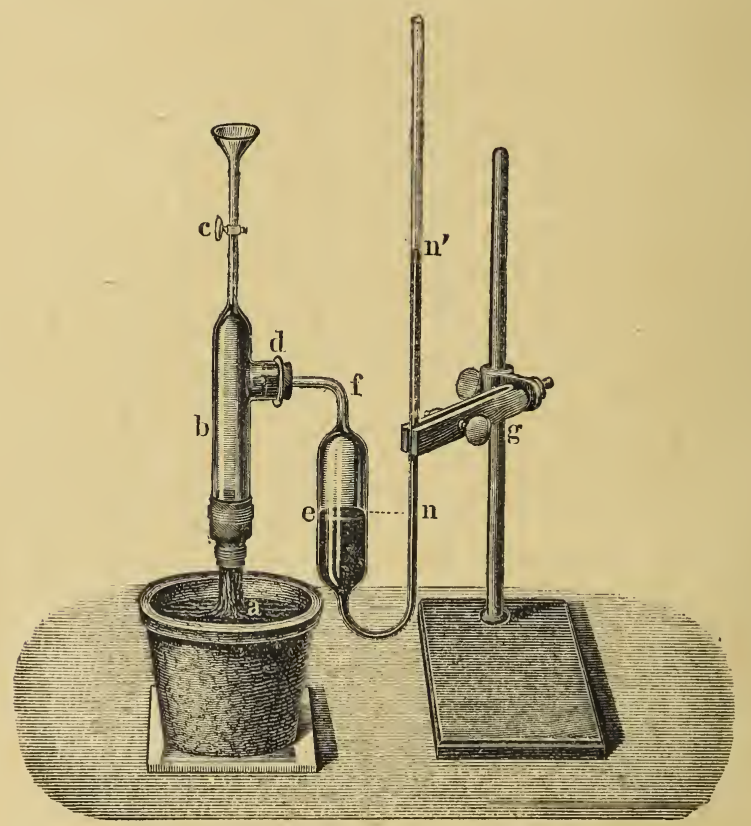

Fig. 82. - Apparcil pour la démonstration de la force d'ascension de la sìve.

Quand les feuilles commencent à apparaître et pendant qu'elles se dévcloppent, la marche de la sève devient plus active; elle est en outre favoriséc par la chaleur ambiante ct l'humidité du sol.

Mais quand les feuilles sont formées, sa marche se ralentit; c'est alors que les tissus nouvellement formés se complètent et prennent plus de consistance. 
Dans un certain nombre d'arbres, outre la montée de la sève de printemps, à laquelle se rapporte ce qui vient d'être dit, il y a une sève d'automne, laquelle coïncide avec le jaunissement des feuilles et la formation des bourgeons qui s'épanouiront en feuilles au printemps suivant; le Peuplier d'Italie, le Tilleul, entre autres, en offrent des exemples bien connus.

Cinuses de la montée de la sève. - Plusieurs théories ont été invoquées pour expliquer le mode de progression de la sève, la force à

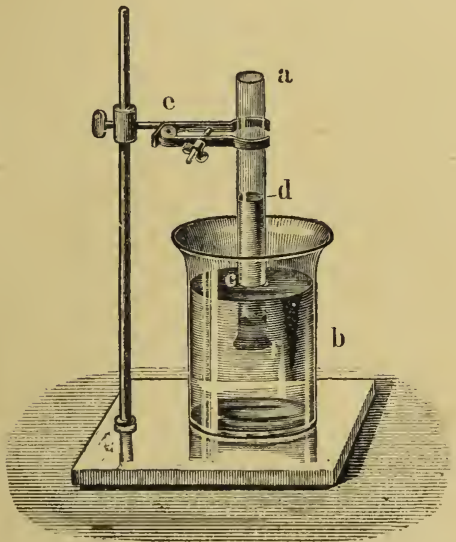

Fig. 83. - Appareil pour la démonstration des phénomènes d'endosmosc. Dans un vase $(b)$, renfermant de l'cau $(c)$, plonge un tube $(a)$, contenant une solution de gomme $(d)$, et fermé à l'extrémité inféricurc par une membrane animale $(f)$. laquelle est dû ce phénomène. Nous allons passer en revue les principales.

10 Endosmose. L'une de celles qui a eu le plus de vogue et l'une des plus satisfaisantes est due au botaniste Dutrochet; elle est toute physique. Ce savant compare ce quise passe dans la Plante aux phénomènes qui s'accomplissent dans l'endosmomètre. On appelle ainsi un appareil composé d'un tube assez large, au moins à l'une de ses extrémités et fermée par une membrane de nature animale. On verse dans cette sorte de récipient un liquide gommé ou sucré et on le plonge dans un vase contenant de l'eau pure; il s'établit aussitôt un double courant, l'un d'endosmose, qui a pour effet de faire pénétrer dans le tube, à travers la membrane qui le ferme, une forte portion de l'eau pure dans laquelle il plonge; l'autre, d'exosmose, en vertu 
duquel une très faible partie de la solution gommée ou sucrée va en sens contraire. Il y a donc, en résumé, augmentation considérable du liquide renfermé dans le tube.

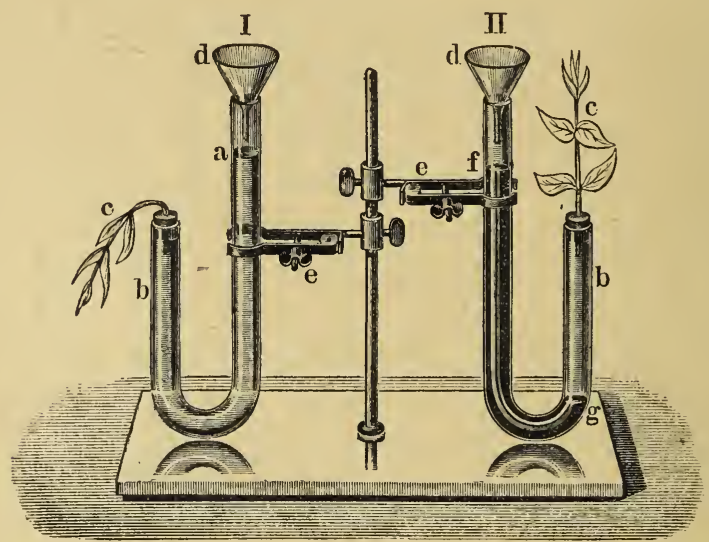

Fig. 84. - Appareil destiné à montrer comment un rameau fétri redevient turgescent en quelques secondes, quand il est mis au contact de l'eau soumise à une certaine pression.

Or, le même phénomène se passerait dans la plante entre deux cellules voisines, lesquelles ne sont séparées que par une mince membrane de nature organique ; celle qui contient un liquide plus aqueux le laisserait échapper en partie dans celle dont les sucs sont plus épais. Or la sève est un liquide essentiellement aqueux contenant une très faible proportion de substances en dissolution, et elle rencontre, en montant dans la plante, des cellules remplies d'un protoplasma épais et possédant un suc intracellulaire chargé de principes solides en dissolution, formé dans la végétation précédente. Toutes les conditions sont donc réunies pour que les phénomènes se passent comme dans l'endosmomètre; par conséquent de proche en proche, d'une cellule à une autre, le liquide est forcé de gagner des parties de plus en plus élevées de la tige.

Ajoutons que, suivant la pression à laquelle est soumis le liquide, son ascension est plus ou moins énergique, 
comme le montre l'appareil représenté dans la fig. 84. Des deux tubes recourbés, I, II, le premier contient seulement de l'eau $(a b)$, l'autre, du mercure $(f g)$ et de l'eau $(g b)$. Les petites branches sont hermétiquement fermées par un bouchon de caoutchouc traversé par un rameau feuillu $(c, c)$. Enfin la grande branche de chaque tube porte un entomnoir $(d)$ par lequel on verse l'eau ou le mercure. Dans les deux cas la surface de section des rameaux est en contact avec l'eau, mais tandis que l'un des rameaux est flétri, l'autre, sous l'influence de la montée de l'eau produite par la pression du mercure qui remplit la grande branche, est rigide et turgescent.

Cette théorie toute physique de l'endosmose est séduisante ; mais il faut remarquer qu'elle ne suffit pas à expliquer entièrement l'ascension de la sève, car on voit celle-ci se modifier si les cellules à travers laquelle elle se fait ont été tuées par une cause qui n’a cependant pas altéré leur constitution physique, ce qui montre bien qu'il y a dans ce fait autre chose qu'un simple phénomène mécanique, mais bien une véritable force qui paraît ne dépendre que de la vie même.

2. Capillarité. On a essayć de remplacer la théorie précédente par une autre de même ordre. On sait que, malgré la pression atmosphérique, l'eau monte dans des tubes capillaires, quand on y plonge leur extrémité. Les cavités des ccllules et des vaisseaux étant extrêmement étroites peuvent être comparées à ces instruments, et plusieurs physiologistes ont voulu voir là la cause de l'ascension de la sève.

$3^{\circ}$ Imbibition. On a également invoqué l'imbibition, c'est-à-dire, cette force d'ordre physique aussi, qui fait monter un liquide, comme cela a lieu dans un ruban vertical dont l'extrémité inférieure serait immergée.

$4^{\circ}$ Évaporation. Il faut signaler, sinon comme cause absolue de l'ascension de la sève, du moins comme cause adjuvante très importante, l'évaporation qui se produit à la surface des feuilles. Par suite de ce phénomène, on comprend qu'un vide tend à se produire dans les cellules qui occupent 
les parties supérieures de la plante, d'où résulte un appel incessant du contenu liquide des cellules situées au-dessous, action qui se fait sentir de proche en proche jusqu'aux cellules des régions les plus inférieures.

On pourrait donc comparer cette force à une sorte de succion ou d'aspiration exercée par les feuilles sur les parties sous-jacentes. On comprend, d'autre part, que l'évaporation agisse sur la montée de la sève d'une façon plus ou moins efficace, suivant l'élévation plus ou moins grande de la chaleur ambiante et la vivacité de la lumière, circonstances qui activent singulièrement l'évaporation.

- En résumé, il faut tenir compte, dans le phénomène de l'ascension de la sève, d'une force particulière, d'ailleurs inconnue, sans doute d'ordre vital, et en outre, de toutes les causes qui viennent d'être passées en revue, auxquelles on doit ajouter la dilatation des gaz mêlés au liquide séveux, et qui bien loin d'exister à l'exclusion l'une de l'autre, paraissent plutôt coexister et se prêter un mutuel appui.

Composition de la sève ascendante. - La sève, qui est d'abord un liquide essentiellement aqueux, se modifie peu à peu, à mesure qu'elle monte dans la tige. Vers les parties inférieures de la plante ce n'est que de l'eau tenant en dissolution des traces d'albumine, de sucre, de gomme et quelques sels. Mais à mesure qu'elle s'élève, elle rencontre des substances diverses formées par les végétations précédentes, les dissout et les emporte avec elle, comme albumine, caséine, sucre, gomme, sels divers, phosphate et tartrate de chaux, sulfate de potasse, acide carbonique libre, etc., de sorte que la sève qui arrive au sommet de la plante est bien plus riche en principes organiques et minéraux que celle que l'on pourrait retirer des parties inférieures.

C'est la sève qui, dans certaines plantes, dissout une telle proportion du sucre accumulé d'avance dans les cellules, qu'elle conslitue une liqueur fort agréable et susceptible de fournir de l'alcool par la fermentation. Ainsi, en faisant quelques incisions au trone des Palmiers 
et mieux encore aux spadices ou inflorescences de ces arbres, on obtient un liquide abondant, qui par la fermentation, devient le vin de Palme, lequel donne par la distillation l'eau-de-vie d'Arack.

Voles sujvies par la sève ascendante. - Il est facile, au moyen d'une expérience que chacun peut répéter, de constater par quelle portion de la tige la sève monte. Si, dans le temps où celle-ci commence à entrer en mouvement on perce avec une vrille les couches extérieures d'un arbre, on voit que l'écorce ne laisse écouler aucun liquide, et que pour obtenir ce résultat, il faut enfoncer l'instrument dans les couches mêmes du bois ; el ici, il y a une distinction à établir entre les arbres dont tout le corps ligneux est tendre, comme sont nos arbres à bois blanc (Peuplier, Saule, etc.) et ceux dont les parties centrales se distinguent des extérieures par une nuance plus foncée et une dureté plus grande pour constituer le cœur ou duramen (Chêne, Noyer). Dans les premiers, la sève monte à travers toute l'épaisseur du bois et surtout par les parties centrales; dans les seconds, elle ne s'élève qu'à travers les couches extérieures, c'est-à-dire l'aubier, la région du cœur étant formée de vaisseaux et de fibres à parois épaisses et imperméables.

Les vaisseaux et les fibres servent aussi bien les uns que les autres à conduire la sève, du moins pendant quelque temps; mais les vaisseaux ne jouent ce rôle que d'une façon temporaire, ou du moins, ils ne renferment un peu plus tard qu'une petite quantité de liquide mélangée d'une grande quantité de gaz, et cela jusqu'à l'entrée de l'hiver suivant, pendant la durée duquel enfin ils ne contiennent plus rien que des gaz.

Remarque. Arrivée dans les feuilles, la sève subit des modifications considérables, sous l'influence d'une triple cause, à savoir: la transpiration, qui s'opère par l'intermédiaire de ces organes, la fonction chlorophyllienne, qui joue un rôle de première importance en fournissant à la nutrition un de ses éléments essentiels, le carbone de l'air, 
enfin la respiration, cette fonction organique fondamentale.

Le moment serait donc venu d'étudier ces différentes points de la physiologie végétale. Mais pour ne pas interrompre notre étude de la circulation de la sève, nous remettrons l'examen de ces trois fonctions après en avoir fini avec celle-ci.

Sève descendante. - La sève, contenant en solution les divers principes qu'elle a rencontrés dans sa marche ascendante, puis profondément modifiée par les phénomènes dont les feuilles sont le siège, porte le nom de sève élaborée, de sève nourricière ou de sève descendante. Elle va, en effet, se répartir des régions supérieures de la plante dans toutes les parties situées plus bas, jusque dans les racines, pour fournir à tous les éléments anatomiques les matériaux de leur nutrition.

Il est bon de faire remarquer que l'expression de sève descendante n'est pas toujours rigoureusement exacte, car la marche de ce liquide peut être différente, ascensionnelle même dans certaines circonstances; c'est ainsi, par exemple, que les sucs nourriciers renfermés dans un tubercule de pomme de terre en germination, s'épuisent peu à peu, et montent dans les jeunes pousses en voie de développement, qui s'élèvent vers l'atmosphère. Il serait donc plus vrai de dire que les sucs élaborés se portent des parties où ils se sont formés ou accumulés, vers celles qui en ont besoin, quelle que soit la direction à suivre. Mais on comprend que dans la généralité des cas la marche soil descendante, puisque l'élaboration se fait d'ordinaire au niveau des feuilles.

Voie suivie Par la SÈve descendante. - De même qu'une expérience bien simple nous a fait voir le chemin que suit la sève ascendante, une autre non moins simple, mais dont le résultat se fait attendre plus longtemps, va montrer par quelle voie elle revient. Il suffit de lier fortement avec un fil de fer la tige d'un jeune arbre: après un an ou deux on verra au-dessus du lien un bourrelet circulaire, qui 
tendra à s'augmenter d'année en arnée, résultat dú manifestement à l'accumulation des sucs nutritifs en ce point, par suite de la difficulté qu'ils trouvent à se frayer un chemin. Les choses iront même au point que le bourrelet cortical finira par surplomber et recouvrir le lien de fer, puis par rejoindre la partie située au-dessous de lui; de la sorte, le fil de fer finira par être caché entre le bois et l'écorce; c'estlà l'explication de la découverte, qui a été faite plusieurs fois, d'objets divers rencontrés dans l'épaisseur même des arbres, tels que clous, cornes de cerf, etc., entièrement masqués par les productions successives de l'écorce.

G'est, en effet, à travers les tissus de la plus grande partie de l'écorce que se fait la descente de la sève, non par les fibres libériennes, dont la paroi est très épaissie, mais par les éléments pourvus de parois minces et ayant un calibre relativement large, surtout les cellules et tubes grillagés si généralement répandus dans l'écorce, et qui méritent pour cela le nom de cellules et de tubes sèveux. (Voy. p. 63.)

La circulation est particulièrement active dans la région interne de l'écorce, c'est-à-dire, au niveau de la zone génératrice ou cambium, à laquelle elle fournit des matériaux abondants pour la formation des cellules, dont la multiplication répétée donne du bois, d'une part, de l'écorce, de l'autre.

La sève concourt d'ailleurs à la formation de lous les autres éléments de la plante et à l'élaboration des substances diverses qui s'accumulent en elle et forment des dépôts plus ou moins abondants, réserves nutritives qui seront employées pendant la période végétative suivante.

Girculation du Protoplasira et du Latex.-Pour ne rien omettre d'essentiel au point de vue de la circulation des liquides dans la Plante, nous devons rappeler qu'outre le double mouvement d'ascension et de descente de la sève, il existe dans chaque cellule en particulier une véritable circulation, qui a été étudiée au Chapitre $\mathrm{I}^{\mathrm{er}}$, page 5 , sous le nom 
de courants protoplasmiques, et i laquelle on donne souvent aussi le nom de rotation ou de gyration du protoplasma. Les poils transparents de certaines plantes, ccux de Tradescantia, notamment, les cellules allongées de la tige des Chara, sont tout particulièrement favorables à ce genre d'observation.

Ajoutons, enfin, qu'il existe dans la plante un autre système circulatoire, qui paraît tout à fait indépendant de celui que nous venons d'étudier longuement; c'est celui des vaisseaux du suc propre ou laticifères, dont la structure a été examinée au Chapitre $1^{\mathrm{er}}$, page 14, et le liquide qu'ils contiennent, au Chapitre II, page 42. Bornons-nous à dire que ces vaisseaux sont le siège de courants, faciles à observer dans les plantes où le latex est coloré, comme celui de la Chélidoine ou Grande Éclaire, où il est d'un beau jaune. On peut voir alors, que les laticifères sont anastomosés entre eux, de façon à former des réseaux à mailles polygonales ou arrondies. Le latex parcourt chaque maille suivant un mouvement circulaire, mais en passant souvent, en partie, dans les vaisseaux qui appartiennent aux mailles voisines.

Transpiration.

Nature de cetre foncrion. - La transpiration, qu'on appelle quelquefois, mais à tor', évaporation, est une fonction d'ordre vilal et non pas un phémonène purement physique comme serait ce dernier. On en a la preuve dans ce fait, que la plante, tant qu'elle est vivante, exhale beaucoup moins de vapeur d'eau que quand la vie s'est éteinte en elle : ainsi une plante qui vient d'être coupée dégage une bien plus grande quantité d'eau que lorsqu'elle était sur pied. 
SiÈge de la transpiration. - Il est facile de démontrer expérimentalement la transpiration des végétaux : il suffit de recouvrir d'une cloche de verre une plante en pot ou une branche d'arbre; la vapeur d'eau qui se dégage se condense et ruisselle en gouttelettes sur les parois de la cloche.Souvent, quand l'atmosphère est chargée d'humidité, le matin, par exemple, les plantes sont couvertes de gouttes d'eau, chaque brin d'herbe en porte une à son extrémité : ce n'est là le plus souvent que le résultat de la transpiraration accomplie pendant la nuit, plutôt que le fait de la condensation de l'humidité de l'air au contact des feuilles. Les produits de la transpiration se répandent dans l'atmosphère surtout par l'intermédiaire des feuilles; mais il ne faut pas croire que cette fonction ait pour siège unique la surface foliaire. Elle s'accomplit, en effet, aussi bien dans l'épaisseur même des organes, là où se trouvent des lacunes ou méats intercellulaires, dans lesquels les cellules exhalent sous forme de vapeur une partie de l'eau qu'elles renferment. Celle-ci gagne de proche en proche la surface des feuilles ou certaines autres parties perméables; si les feuilles sont, en effet, le lieu principal de celte exhalation, les parties jeunes de la tige et des rameaux prennent part aussi au phénomène.

La transpiration est surtout abondante dans les points où les stomates sont nombreux; aussi est-elle plus aclive à la face inférieure des feuilles qu’à leur face supérieure; il ne faudrait pas croire toutefois qu'elle ne s'accomplisse que là où il existe des stomates; elle se fait sans doute à travers des pores invisibles, au niveau de tous les organes qui ne sont pas recouverts d'une couche subéreuse trop épaisse ou d'une cuticule imperméable.

Activité de la transpiration. - La quantité d'cau évaporée par les plantes est considérable. Le savant physiologiste Hales, en expérimentant sur le Grand soleil (Helianthus annuus), nota une exhalation de près de 1000 grammes d'eau, dans un intervalle de 12 heures, pendant une journée sèche et chaude. On comprend, d'après cette donnée, quelle 
énorme quantilé de vapeur d'eau doit répandre dans l'atmosphère une végétation puissante, pourquoi les pays, où elle est luxuriante, sont d'une grande humidité, comment, enfin, le boisement d'une contrée sèche el aride peut en modifier le climat: les forêts, en jetant dans l'atmosphère d'énormes quantités de vapeur d'eau, amènent son refroidissement, et les vapeurs de l'air se condensant retombent en pluie. Cette émission de vapeur d'eau est telle que les aéronautes, en passant à des centaines de mètres audessus des forêts, ressentent une impression de froid causée par ces épais tourbillons qui arrivent jusqu'à eux.

Conditions QUi nodifient cette fonction. - Plusieurs circonstances modifient la transpiration des végétaux. Ainsi elle est activée par une lumière vive, par la chaleur, bien que, cependant, à de très basses températures, à $0^{\circ}$ et même á - $25^{\circ}$ elle s'exerce encore, enfin par l'agitation de l'air. Au contraire, elle est ralentie par l'humidité du milieu ambiant et par l'obscurité; c'est pourquoi une plante en pot se conserve bien plus longtemps fraîche, sans arrosage, quand elle est renfermée dans un lieu sombre que lorsqu'elle est exposée aux rayons du soleil, même en prenant les précautions voulues pour éviter l'évaporation de l'humidité de la terre où elle végète.

D'autre part, les plantes herbacées, celles qui sont formées d'un parenchyme mou, spongieux, transpirent beaucoup plus abondamment que celles dont le tissu est sec et serré; les arbres verts transpirent moins que ceux à feuilles caduques, aussi végètent-ils facilement dans les pays chauds, où l'eau est rare en été.

Enfin on a observé qu'il se produil dans cette fonction une variation diurne, telle que le maximum d'évaporalion aurait lieu de midi à 2 heures et le minimun pendant la nuit. 


\section{GHAPI'TRE VII}

\section{Suite des Fonctions de nutrition.}

RESPIRATION ET FONCTION CHLOROPHYLLIENNE. Différences fondamentales entre ces deux fonctions. Étude comparée de la respiration et de la fonction chlorophyllienne. Conséquences de la respiration : production de chaleur; production de lumière. - DIGESTION ET assimilation. Parties de la plante où s'opère la digestion. Plantes carnivores. Nature des ferments digestifs. - Assimilation. - Réserves nutritives. - Classification des substances assimilées.

Respiration et Fonction chlorophyllienne.

Différences fondanentales entre ces deux fonctions. - Sous le nom de respiration on a longtemps confondu deux ordres de phénomènes tout à fait différents : l'un consiste dans l'absorption de l'acide carbonique de l'air, dont le carbone reste fixé dans la plante, tandis que l'oxygène retourne dans l'atmosphère; l'autre consiste, au contraire, dans l'absorption et la fixation par la plante de l'oxygène de l'air, et le rejet d'une certaine quantité d'acide carbonique.

La distinction de ces deux fonctions n'est établie avec quelque précision que depuis une époque récente, bien que, depuis assez longtemps, l'observation du rôle joué par les feuilles ait fait soupçonner la vérité. Ainsi, dans la première moitié du xvnl ${ }^{\mathrm{e}}$ siècle, Hales disait : "Les feuilles servent aux végétaux comme les poumons aux animaux. » Priestley observa que des feuilles fraîches plongées dans de l'eau de source et exposées au soleil dégageaient des 
bulles de gaz qu'il reconnut être de l'oxygène (qu'on appelait alors air déphlogistiqué, air vital); il en conclut que les plantes purifiaient l'atmosphère pour le rendre respirable aux animaux.

Ingenhouz remarqua que ces mêmes plantes qui, au soleil, purifient l'air, le vicient à l'abri de la lumière, en exhalant un gaz bien différent, l'acide carbonique. Enfin Sénebier montra que l'oxygène dégagé par la plante exposée au soleil est le résultat de la décomposition de l'acide carbonique de l'air ou bien de celui que les racines ont puisé dans le sol.

De ces observations il résulte que les végétaux se comportent vis-à-vis de l'atmosphère de deux façons bien différentes. Dans l'un des cas, en effet, il s'agit d'une fonction d'ordre purement chimique, à savoir la réduction de l'acide carbonique emprunté à l'air; dans l'autre, une fonction vitale de premier ordre entre en jeu, la respiration proprement dite.

Ce second ordre de phénomènes peut être complètement assimilé à la respiration des animaux, puisqu'il consiste dans l'absorption de l'oxygène de l'air, lequel se combine ensuite dans les tissus mêmes de la plante avec certains principes hydro-carbonés, pour former de l'acide carbonique destiné à être rejeté au dehors (1).

Il n'y a pas d'antagonisme fonctionnel entre la Plante et l'Animal.

Cette étude montre que l'antagonisme fonctionnel que l'on a voulu jadis établir entre les animaux et les plantes n'existe pas en réalité. La respiration des uns et des autres a pour résultat final de vicier l'air atmosphérique en lui prenant de l'oxygène et lui rendant de l'acide carbonique. Mais nous nous hâterons d'ajouter que la composition normale de l'atmosphère est néanmoins maintenue grâce aux végétaux, puisque chez eux, à côté de la respiration proprement dite existe une autre fonction, dont le rôle est de prendre l'acide carbonique de l'air et de rejeter de l'oxygène en échange; et même l'activité de cette seconde

(1) Voy. Anatomie et Physiologie animales, par le docteur P. MaISONNEUVE, 1885, p. 199. 
fonction est telle qu'elle compense la viciation de l'atmosphère causée par la respiration proprement dite tant des plantes que des animaux.

Nous allons voir maintenant, que non seulement le but de la respiration est différent de celui de l'action chlo-rophyllienne, mais encore que le degré de généralité de ces deux fonctions, les organes par lesquels elles s'opèrent, et par conséquent, leurs phénomènes intimes, ne sont pas les mêmes.

Étude comparée de la Respiration et de la Fonction CHLOROPHYLLIENNE. - La respiration offre un caractère de généralité absolue; elle existe chez toutes les plantes, et s'opère aussi bien à la lumière vive du jour que pendant l'obscurité de la nuit; c'est une fonction d'ordre vital, dont la Plante ne peut pas plus se passer que l'Animal lui-même.

La fonction chlorophyllienne est bien loin de nous offrir la même généralité; d'abord elle ne s'opère pas chez toutes les plantes, car celles qui sont dépourvues de matière verte ou chlorophylle, comme les Champignons, ne peuvent jamais la présenter; chez les autres, elle ne se produit que dans certaines circonstances déterminées, car elle a besoin, pour s'exercer, de la lumière solaire et se suspend complètement dans l'obscurité. Aussi, lorsqu'on laisse une plante dans un lieu très sombre pendant un certain temps, la chlorophylle n'agit plus, disparaît même, et ses tissus perdent leur consistance; la plante, s'est, comme on dit, étiolée.

Nous venons de voir que, dans les plantes, la chlorophylle a seule le pouvoir de décomposer l'acide carbonique; il en Siège de ces deux fonctions. résulte donc que les feuilles sont les organes essentiels, presque exclusifs de cette opération, car c'est en elles surtout que la matière verte est abondante.

La respiration, tout en s'exerçant également au niveau des feuilles, présente son maximum d'activité dans les parties colorées autrement que par la chlorophylle, c'està-dire dans les fleurs, notamment les étamines. Ainsi on a observé que la fleur d'une Passiflore (Passiflora serratifolia)

La respiration s'exerce vec bien plus de continuité que la fonction chlorophyllienne. 
absorbe, en 24 heures, dix-huit fois et demie son volume d'oxygène, tandis que, dans le même temps, les feuilles de cette plante n'en prennent que cinq fois environ leur volume.

Les étamines étant les parties de la fleur où cette absorption de l'oxygène est le plus active, on comprend facilement pourquoi les fleurs simples en consomment plus que les fleurs doubles.

Conditions qui activent ces deux fonctions.

Quoique la respiration s'exerce en tout teinps, elle s'opère dans les fleurs avec une bien plus grande activité à la lumière vive du soleil, qu'à l'ombre. On comprend d'appès cela qu'il soit dangereux de séjourner dans un appartement où se trouvent réunies beaucoup de fleurs fraîches, surtout si le soleil y a accès, car en même temps que l'oxygène est pris par elles en grande quantité, l'acide carbonique est rejeté en proportion corrélative.

Au contraire, des plantes vertes placées dans les mêmes conditions auraient pour effet de purifier l'air, par suite de l'absorption de l'acide carbonique et de l'exhalation de l'oxygène sous l'influence de la chlorophylle.

La respiration dans les différents organes des plantes et chez les végétaux inférieurs.
Les parties les plus différentes de la Plante respirentégalement l'oxygène, par exemple, la tige, les fruits, alors qu'ils sont mûrs; les bourgeons, au moment où ils s'épanouissent en feuilles, donnent lieu à un dégagement abondant d'acide carbonique, corrélatif à une absorption considérable d'oxygène. Il en est de même des graines au moment de la germination, sujet qui sera étudié en détail au Chapitre xır.

Les plantes, même sans chlorophylle, et notamment les Champignons, absorbent également l'oxygène et dégagent de l'acide carbonique.

Enfin, les organismes végétaux les plus inférieurs, comme les Levures, les Ferments, les Bactéries, etc., respirent aussi. Mais, selon M. Pasteur, tandis que les uns respirent, comme toutes les autres plantes, l'oxygène de l'air, ce qui leur a valu le nom d'aérobies, (ảńp, air, ßíos, vie), les autres sont tués par ce même oxygène, quand ils sont mis en sa présence, et ne respirent que celui qu'ils enlèvent à certaines matières organiques au contact desquelles ils 
se trouvent, et qu'ils décomposent par là même, d'oủ leur nom d'anaérobies ( $\alpha$ priv., ג'ńp, air, ßíos, vie).

Les phénomènes intimes de la respiration s'opèrent, bien entendu, dans l'intérieur des cellules; leur protoplasma

Nature

des fonctions respiratoire et chlorophyllienne. est le véritable siège de ce grand acte vital; mais si nous connaissons les résultats, nous sonmes bien peu renseignés sur la façon exacte dont les choses se passent.

Quoi qu'il en soit, l'oxygène respiré se combine avec les substances hydro-carbonées contenues dans la plante, et la combustion lente ainsi produite dans le sein des tissus végétaux détermine une production de chaleur, d'acide carbonique et de vapeur d'eau, qui se répandent au dehors. Une des conséquences de cette fonction est donc manifestement pour la plante une perte de substance.

La fonction chlorophyllienne n'est pas moins difficile à connaître dans son essence. On sait toutefois, que, sous l'influence de la lumière, le protoplasma des cellules à chlorophylle réduit l'acide carbonique, dont il retient le carbone et laisse dégager l'oxygène. Contrairement à ce que vient de nous montrer la fonction respiratoire, il y a donc ici un véritable gain pour la plante, une augmentation de substance. Il paraît probable que ce carbone est ensuite combiné avec de l'eau pour former un hydrate de carbone ( $\mathrm{C}, \mathrm{HO}$ ), qui est ordinairement de l'amidon ou fécule; aussi voit-on cette substance exister abondamment là où il y a de la chlorophylle, tandis qu'elle fait défaut quand celle-ci est elle-même détruite, par suite d'un séjour prolongé à l'obscurité. Dailleurs, si l'amidon disparaît complètement de la plante, celle-ci non seulement ne s'accroît plus, mais elle meurt bientôt. C'est ce qu'on peut observer pendant la germination des graines dans un local sombre : tant que la provision de fécule renfermée dans la graine n'est pas épuisée, la jeune plante grandit; mais quand toute cette réserve nutritive a été absorbée, elle ne tarde pas à mourir, à moins qu'on ne l'expose alors à la lumière, sous l'influence de laquelle il se formera promptement une nouvelle provision d'amidon.

On voit, d'après cela, combien est grande dans la vie de 
la plante, l'importance de la chlorophylle et de la fonction qu'elle est appelée à jouer, puisque d'elle dépend l'élaboration de l'amidon, l'un des aliments nutritifs les plus indispensables à l'organisme végétal.

Conséquences de la Respiration ; Production de chaLEUR. - Toute combustion produit de la chaleur; mais quand la première est très lente, la seconde est très faible; c'est ce qui a lieu le plus souvent dans les plantes, de sorte que l'élévation de température est difficilement appréciable. Il existe cependant des circonstances où elle devient manifeste, par exemple, dans la respiration de certaines fleurs, dans lesquelles de Saussure a vu l'absorption de l'oxygène atteindre un volume égal à trente fois le leur. On la met facilement en évidence quand un grand nombre de fleurs sont portées sur un même pédoncule, comme c'est le cas des Aroïdées, par exemple. Lamarck, en 1777, avait observé ce phénomène sur un Arum; Sénebier nota une élévation de $9^{\circ}$ c. au-dessus de l'air ambiant, produite par une plante du même genre; un thermomètre placé entre plusieurs Arum de Madagascar, en pleines fleurs, dénota une chaleur de $25^{\circ}$ au-dessus de la température ambiante. Des phénomènes analogues ont été observés sur nombre d'autres plantes, telles que Nénuphars, Courges, Cactus, etc.

Les graines en germination produisent également de la chaleur; il est de connaissance vulgaire, en effet, que les grandes quantités d'orge que l'on fait germer dans des espaces clos, pour la préparation du malt qui sert à la fabrication de la bière, dégagent une forte chaleur.

Un appareil bien simple permet de noter le degré de chaleur produit par des fleurs en voie d'épanouissement ou des graines en germination. On place, par exemple, une poignée de capitules de Scabieuses ou mieux encore, des haricots, dans un entonnoir engagé dans le goulot d'un flacon qui contient une certaine quantité d'une solution de soude ou de potasse, destinée à absorber l'acide carbonique qui se dégagera. Les semences de Haricot, par exemple, étant humectées d'eau, on recouvre le tout d'une cloche 
percée d'un orifice supérieur fermé seulement par un bouchon de coton à travers lequel passe un thermomètre qui plonge au milieu des graines, et qui montre que la température de l'air de la cloche s'élève de plus d'1º sous l'influence de la germination.

Phosphorescence. - Dans quelques circonstances l'absorption de l'oxygène est accompagnée d'une production de Jumière. Ainsi on connaît, en Provence, un Champignon, l'Agaricus olearius, qui montre nettement ce phénomène; et, ce qui prouve que celui-ci résulte bien de la respiration, c'est qu'il se suspend dès que cette fonction est supprimée, comme cela a lieu quand on place la plante dans un milieu privé d'oxygène. Plusieurs autres plantes inférieures sont également phosphorescentes; citons l'Agaricus igneus, d'Amboine, l'Agaricus noctilucens, de Manille, l'appareil végétatif de l'Agaricus melleus, dont on avait fait le genre Rhizomorpha (1).

Digestion. - Assimilation.

Parties de la plante ou se fait la digestion. Plantes Carnivones. - Pas plus que l'Animal, la Plante ne peut se Nécessité nourrir des substances alimentaires que, par un travail antérieur, elle a puisées dans le sol ou dans l'air, et même de celles qu'elle a accumulées en elle, si elle ne leur fait au préalable subir une certaine élaboration, ayant pour effet, de les modifier chimiquement, de les rendre solubles et

(1) Comparez ces phénomènes avec ceux de même ordre présentés par certains animaux, appelés, pour cette raison, phosphorescents. (Voyez Anatomie et Physiologie animales, par le docteur P. MaIsoNNEUVE, 1885, p. 230.) 
aptes à faire ensuite définitivement partie des tissus, si elle n'opère, en un mot, une véritable digestion.

Digestion

\section{intracellulaire.}

Le plus souvent, cette digestion s'accomplit dans l'intimité des organes de la plante et il nous est bien difficile d'en suivre les phases et les opérations dans tous leurs détails; mais nous en connaissons tout au moins les principaux résultats.

Digestion au niveau des racines.

Digestion au niveau des feuilles. Plantes carnivores.
On sait de plus que la plante a la possibilité d'opérer une véritable digestion en dehors de son organisme même, par l'intermédiaire de certaines de ses parties, telles que la racine et lles feuilles. La racine a en effet la propriété de sécréter dans la région voisine de son extrémité une substance acide. L'existence de cet acide peut se constater de plusieurs façons : en faisant croître des racines au contact d'une plaque de marbre bien polie, on voit que les extrémités délicates de ces organes s'y gravent pour ainsi dire, et y laissent leur empreinte, la partie superficielle du marbre ayant été dissoute dans ces points par suite de la sécrétion d'une petite quantité d'acide carbonique; en outre, le papier de tournesol vient confirmer d'une façon non équivoque la nature chimique de cette sécrétion. Grâce à elle, il y a véritablement digestion des phosphates, des sels de potasse, d'ammoniaque, qui se trouvent dans le sol, etc., et qui sont ainsi rendus solubles et susceptibles d'être absorbés par les racines.

Il y a quelques années, Ch. Darwin a montré par un grand nombre d'observations, que plusieurs plantes ont la faculté de digérer au moyen de leurs feuilles ou de certains autres organes, des substances azotées, telles que de petits fragments de viande, du blanc d'œuf, des insectes, d'où le nom de plantes carnivores, sous lequel on les a désignées (1).

(1) C'est au naturaliste anglais Ellis, que l'on doit la première observation scientifique des propriétés singulières de ces plantes; il les mentionne dans une lettre écrite à Linné en 1768, au sujel du Dionxa muscipula. Quant au terme de plantes carnivores, il ful employé pour la première fois par Diderot, qui avait pensé que, non seulement certaines plantes s'emparent des insectes, mais encore les digèrent et les absorbent. 
Parmi ces plantes singulières, les unes retiennent les insectes qui viennent se poser sur elles, simplement au moyen d'une sécrétion visqueuse fournie par les glandes dont leurs feuilles sont pourvues ; tel est le cas des Drosophyllum, Roridula, Byblis. Le premier habite le Portugal et se plaît sur le flanc des collines desséchées;

Drosophyllum; Roridula;

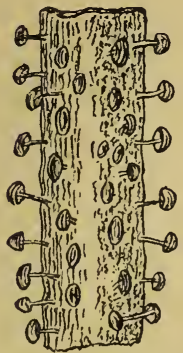

Fig. 85. - Portion de feuille très grossie de Drosophyllum. sa qualité d'attrape-mouche est si bien connue, que les paysans le suspendent dans leurs maisons pour diminuer le nombre de ces incommodes volatiles; ses feuilles linéaires sont garnies de très nombreuses glandes pédiculées terminées par un bouton qui sécrète un liquide visqueux ; l'insecte qui vient s'y poser est bientôt englué, et plus il s'agite, plus il est couvert de cette sécrétion. Le second, qui est du Cap de Bonne-Espérance, atteint une hauteur de plusieurs pieds; ses feuilles également linéaires, sont aussi pourvues de glandes pédiculées, sortes de tentacules dont les uns très longs et les autres courts sécrètent pareillement un liquide visqueux. Le troisième, que l'on rencontre en Australie, offre une disposition analogue au précédent.

D'autres plantes carnivores retiennent les insectes ou tout autre objet mis à lcur contact, au moyen, à la fois, d'une sécrétion visqueusc et de certains mouvements des feuilles ou des espèces de tentacules qui y sont fixés et qui se recourbent pour emprisonner la victime; c'est ce que l'on remarque, par exemple, dans le Drosera. Cette petite plante, appelée encore Rossolis (rosée du soleil), qui croîl dans les endroits marécageux, au milieu des Mousses, des Sphaignes, est fort répandue tant dans l'ancien monde qu'en Amérique; ce genre compte d'ailleurs une cinquantaine d'espèces. On peut facilement observer ce qui se passe sur le Drosera rotundifolia, commun dans notre pays. C'est une petite plante, pourvue seulement de quelques feuilles, 2 à 5 ou 6 , un peu plus larges que longues

Bylulis.

Drosera. 
et dont toute la face supérieure est garnie de filaments ou tentacules, dont les plus longs occupent les bords de la feuille; tous sont renflés en un bouton glandulaire. Chacun
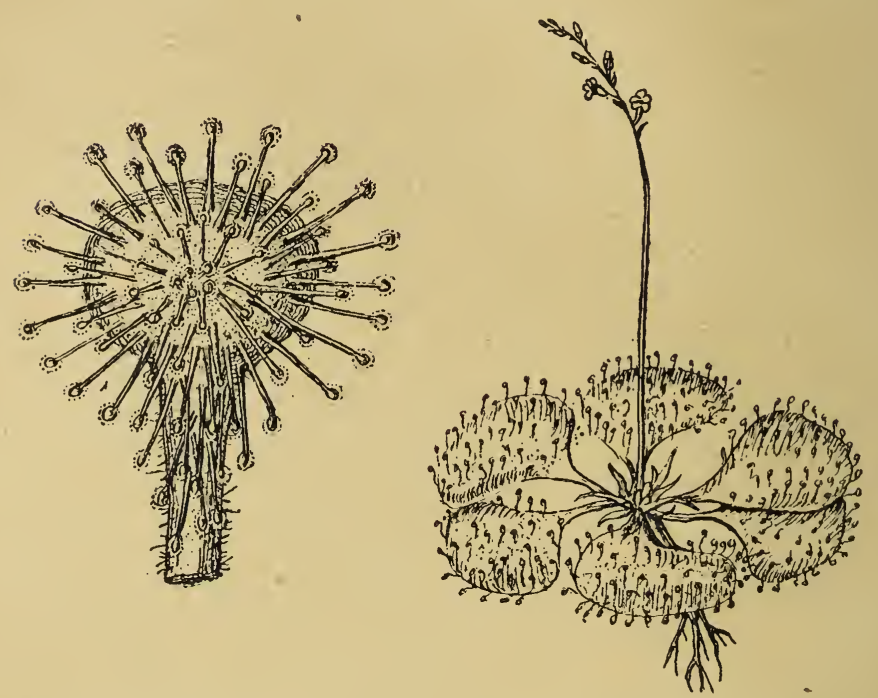

Fig. 86. - Drosera rolundifolia. A droite, vue d'ensemble de la plante, grandeur naturelle. - A gauche une feuille très grossie.

de ceux-ci étant entouré d'une gouttelette d'un liquide visqueux qui brille au soleil comme une petite perle, cette disposition a valu à la plante son nom de Rossolis. Ces poils glandulaires, de structure complexe, ont la remarquable propriété de s'infléchir, de se courber vers le centre de la feuille, sous l'influence de quelque excitation venue du dehors. Qu'un insecte vienne se poser sur une de ces feuilles, ses pattes s'engluent; il cherche à s'envoler, mais ses ailes touchent à leur tour le liquide visqueux, et sous l'influence de l'excitation causée par ses mouvements, les tentacules de la feuille se recourbent sur lui, l'enveloppent et le retiennent prisonnier; ce sont d'abord les plus voisins qui se mettent en mouvement, puis l'excitation se commu- 
niquant de proche en proche, les tentacules les plus éloignés s'inclinent à leur tour. Tout cela demande un temps fort long, de une à plusieurs heures, en général. Après un temps qui varie de 1 à 7 jours ou un peu plus, les tentacules se relèvent, la feuille reprend son aspect ordinaire el se trouve prête à recommencer son manège; mais dans l'intervalle, l'insecte a disparu, à l'exception de ses parties cornées, de ses téguments solides. Si au lieu d'un insecte on y a placé un petit morceau de blanc d'œuf cuit ou de viande, il n'en reste plus trace.

C'est qu'en effet le Drosera a la propriété de dissoudre, de digérer les substances animales et ensuite de les absorber. Cette digestion se fait grâce à la sécrétion des glandes, qui devient très acide, tandis que dans l'état de non activité elle ne l'est pas ou l'est très peu. Ajoutons qu'un objet non azoté ou même inorganique, comme un petit fragment de verre, un grain de sable, provoque les mêmes mouvements et la même sécrétion acide; mais alors la feuille

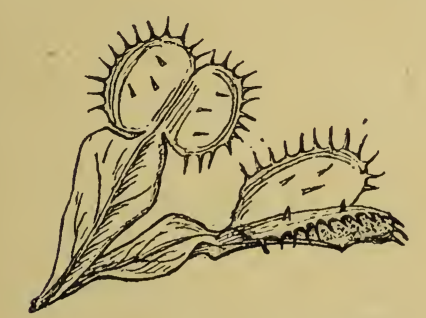

Fig. 87. - Deux feuilles de Diona'a muscipula.

reprend bien plus vite sa position première qu'au cas où on lui offre une substance qu'elle peut digérer.

Enfin, il est d'autres plantes carnivores telles que la Dionée gobemouche (Dioncea muscipula), l'Aldrovandia, qui s'emparent des insectes, grâce seulement à la rapidité des mouvements de leurs feuilles.

Le Dioncea muscipula, de la famille des Droséracées, est une plante des plus étonnantes; on la trouve seulement dans l'est de la Caroline du nord, où elle habite les endroits marécageux. Chaque feuille est formée de deux lobes ovalaires disposés à peu près à angle droil; au milieu de chacun d'eux se voient trois petites épines placées en triangle et d'une extrême irritabilité, 'car au moindre attouchement

Dionara. 
elles déterminent le ploiement de la feuille, dont les deux lobes viennent aussitột s'appliquer l'un sur l'autre. En

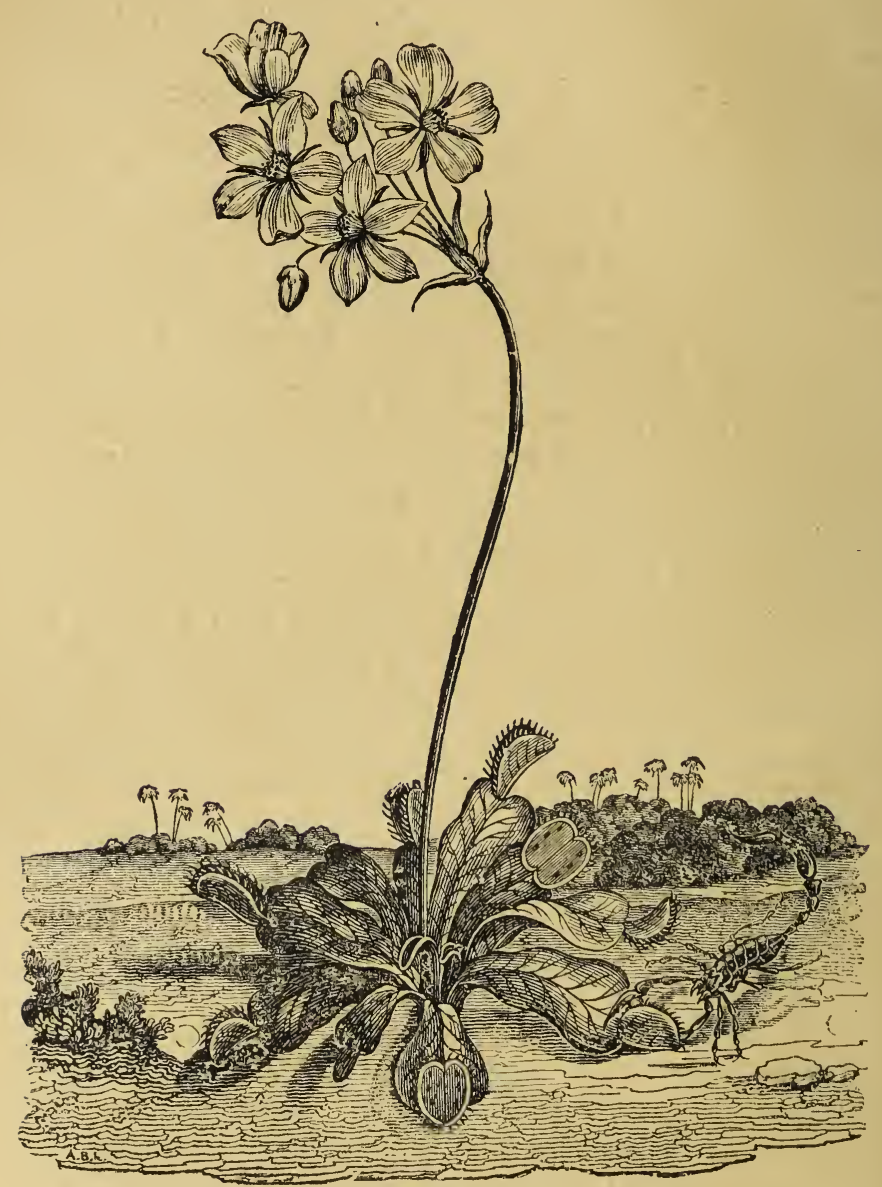

Fig. 88. - Dionæa muscipula; vue d'ensemble de la plante.

outre, les bords de la feuille sont armés d'assez longs piquants qui s'entrecroisent quand elle se ferme et forment une sorte de grillage, qu'un insecte même assez gros ne 
peut guère franchir. Enfin la surface de la feuille est pourvue d'un très grand nombre de petites glandes, d'une teinte rouge, portées par de courts pédicules. Ces glandes, contrairement à celles de Drosera, ne sécrètent un liquide que lorsqu'elles se trouvent au contact d'une substance azotée, qu'elles peuvent par conséquent dissoudre et absorber ; on peut constater, en outre, que le liquide est encore plus acide que celui de la plante précédente.

Ajoutons que les pluies et le souffle du vent ne sont pas, paraît-il, des excitants de nature à provoquer la sécrétion et les mouvements des feuilles de la Dionće, alors que tout autre excitant détermine la brusque fermeture de ses valves; le Drosera a également donné lieu à celte observation.

Le genre Pinguicula, dont une espèce est commune dans

Pinguicula. les endroits humides des pays montagneux, n'est pas moins

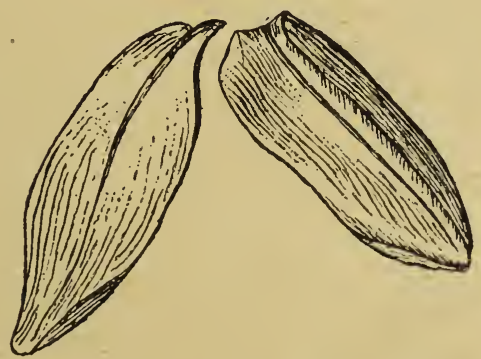

Fig. 89. - Deux feuilles de Pinguicula, celle de gauche complètement étaléc; celle de droite repliée dans toute la longueur d'un de ses bords.

extraordinaire. Ses feuilles sont oblongues, assez épaisses, d'un pouce et demi environ de longueur, avec des bords un peu recourbés et la face supérieure garnie de poils glandulaires sécrétant un liquide très visqueux. Sous l'influence de la légère irritation causée par un insecte, la feuille se replie parallèlement à son bord, de façon à recouvrir le petit animal, en même temps que la sécrétion devient fort abondante. Des substances inorganiques, de petits fragments de verre, par exemple, n'excitent nullement ou que très peu la sécrétion, qui, en tout cas, n'est alors jamais 
acide, tandis que le contact de substances azotées provoque une sécrétion abondante et très acide. Les matières animales ne sont pas seules à produire ce résultat : le pollen, les débris de feuilles ou d'autres parties des végétaux voisins déterminent la sécrétion acide et sont digérés, de sorte que cette pelite plante est, on peut le dire, à la fois carnivore et herbivore.

Aldrovandia. L'Aldrovandia, petite espèce de la mème famille, propre aux eaux douces, est pourvue de feuilles bilobées qui, ordinairement fermées, s'entr'ouvrent seulement à la manière des valves d'une moule, quand la température est assez chaude; mais alors, si un petit insecte aquatique se risque sur ses bords, ceux-ci se rapprochent aussitôt et le retiennent prisonnier. La partie concave des lobes porte des glandes et de long poils, sortes d'organes de sensibilité (1) déterminant quand ils sont touchés, l'occlusion de cette espèce de coquille.

Utriculaires. A côté de cette plante, on pourrait placer les Utriculaires

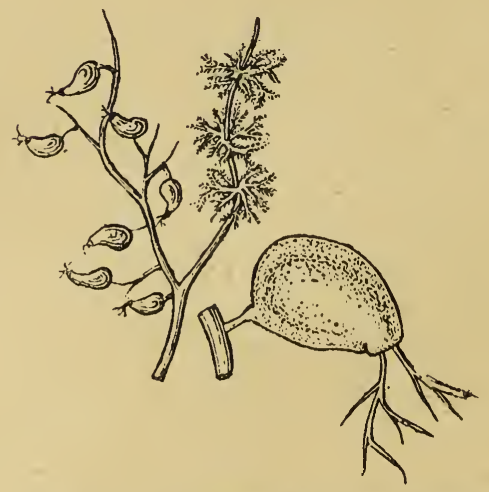

Fig. 90. - Utriculaire. A gauche, vue d'ensemble d'un rameau de la plante portant des ascidies et des feuilles. A droite, une vésicule ou ascidie trìs grossie.
(Utricularia), également aquatiques, dont les feuilles linéaires, profondément découpées, sont pourvues de nombreuses petites vésicules ou ascidies

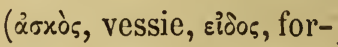
me), longues de deux millimètres et demi. Chacune de celles-ci offre à l'une de ses extrémités, garnie de longs poils, une fente fermée par une valve, laquelle est douée d'une irritabilité telle, que le contact du plus petit vermisseau la

(1) Voyez au sujet de ce qu'on doit entendre par sen sibilité chez les végétaux, le Chap. xiv. 
fait s'infléchir, et que du même coup la petite proie est précipitée dans la cavité intérieure qui va être son tombeau. Cette cavité, garnie de prolongements ou poils rameux, est ordinairement pleine d'eau et d'air. On a cru d'abord que le rôle de ces vésicules était de faire flotter la plante; mais depuis on s'est assuré qu'elles n'étaient nullement nécessaires à ce résultat et que leur fonction véritable est de capturer les insectes; presque toutes, en effet, en contien-

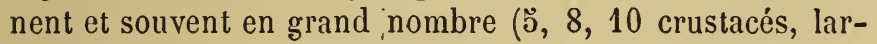
ves, etc.). Ces utricules n'ont pas la propriété, comme les feuilles des plantes examinées jusqu’ici, de digérer les substances animales, qui y ont pénétré ; mais ces substances se décomposent à la longue, et c'est seulement alors, pa raît-il, que leur masse est peu à peu absorbée, la décomposition étant sans doute activée par quelque ferment sécrété par les glandes répandues dans les vésicules.

On pourrait allonger la lisle des plantes regardées comme carnivores; les exemples précédemment cités sont les plus frappants et suffisent à faire connaître celte remarquable particularité.

Nature des ferments digestifs. - Nous avons vu que toutes les plantes digèrent dans l'intérieur de leurs cellules, la plupart aussi par l'intermédiaire de leurs racines, quelques-unes par leurs feuilles ou certains organes qui en dérivent. Nous devons étudier maintenant quels sont les agents chimiques de cette digestion. La fonction digestive s'exerçant sur des substances variables, cet agent doit, lui aussi, être différent suivant les cas.

La plante a la propriété de digérer des substances féculentes, des substances sucrées, des substances grasses et des substances albuminoïdes; il y a de même quatre sortes de principes digestifs ou ferments.

Le ferment de l'amidon ou fécule, l'un des principaux aliments des plantes, est la diastase, que l'on a découverte d'abord dans l'orge en germination. Sous son influence, l'amidon se dédouble en dextrine et en glycose, et finalement en glycose soluble et absorbable; c'est grâce à elle

Diastase, ferment des substances féculentes. 
que le tubercule de pomme de terre en germination digère l'amidon qui le compose presque entièrement, pour le faire servir, une fois modifié, à la nourriture et à l'accroissement des jeunes tiges qui en partent.

Suivant les chimistes, cette diastase végétale ne diffère aucunement de la diastase animale, l'un des éléments importants de la salive, et elle joue chez la Plante le même rôle que dans l'Animal, c'est-à-dir'e, qu'elle transforme l'amidon, insoluble, en glycose soluble.

Ferment inversil.

Le sucre de canne ou saccharose, c'est-à-dire celui que fournit la tige de la Canne à sucre, la racine de la Betterave, elc., ne peut pas servir directement à la nutrition de la plante; il doit être converti d'abord en sucre de raisin ou glycose et en lévulose (sucre incristallisable), substances qui, mélangées l'une avec l'autre, en proportions égales, constituent le sucre interverti. Le principe qui opère ces changements porte le nom de ferment inversif. La même opération se passe dans le corps des animaux pour transformer de même la saccharose en sucre interverti; c'est le suc intestinal qui contient ce ferment spécial.

Ferment émulsif.

Les substances grasses, aussi bien dans les végétaux que dans les animaux, ne peuvent être absorbées par les cellules si elles n’ont pas été d'abord émulsionnées, c'est-à-dire, divisées en gouttelettes d'une extrême finesse.Si l'on broie, par exemple, des graines oléagineuses dans de l'eau, on voit la matière grasse de celles-ci se diviser en une infinité de globules huileux d'une petitesse incroyable; ce que l'on produit ainsi artificiellement, une substance particulière l'accomplit naturellement dans la plante, c'est le ferment émulsif. Ce ferment est en même temps saponifiant, c'est-àdire, qu'il dédouble les corps émulsionnés en acides gras et en glycérine, résultat identique à celui que l'on obtient dans la fabrication des savons, d'où le nom de saponification.

Ce même ferment existe dans le suc pancréatique des animaux.

Pepsine, ferment des substances albuminoïdes.
Enfin, les plantes renferment presque toujours une certaine proportion de substances azolées, car elles se nourrissent, en partie, d'aliments albuminoïdes. Mais ces corps 
ne sont pas directement assimilables et susceptibles de faire définitivement partie de l'organisme végétal; ils doivent être d'abord assez profondément modifiés, passer à l'état de peptone (1), phénomène d'ailleurs assez mal connu dans son essence. Ce qui est acquis, c'est que ce résultat est dû, dans les plantes, comme dans le tube digestif des animaux, à l'action de la pepsine, ferment de nature azotée, qui existe dans le suc gastrique. Le suc contenu dans les laticifères de certains végétaux renferme de la pepsine ; tel est celui du Papayer ou Carica papaya, dont on a pu l'extraire, et que l'on prescrit comme médicament aux personnes dont la digestion se fail mal, au niême titre que la pepsine que l'on retire du suc gastrique des animaux (2). Cette pepsine digère les substances albuminoïdes dans les cellules des plantes, et c'est à elle que l'on croit devoir rapporter les phénomènes de digestion qui s'opèrent à la surface des feuilles des plantes carnivores. Pour que son action puisse s'exercer, il est nécessaire que les aliments soient mis en présence d'un acide; or nous avons vu que la sécrétion des glandes dont - ces feuilles sont pourvues est très nettement acide; toutes les conditions nécessaires à la digestion des substances azotées ou albuminoïdes se trouvent donc là réunies (3).

Assimilation. - Chez les végétaux comme chez les animaux, l'assimilation est la fonction qui a pour effet de rendre les substances absorbées semblables aux tissus mêmes de l'organisme, afin qu'ils puissent en faire désormais partie intégrante. Elle s'exerce soit sur des substances qui viennent d'être puisées au dehors, soit sur des matériaux introduits depuis un temps variable dans la plante, mais restés jusque-là sans emploi.

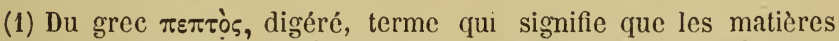
parvenues à cet état sont immédiatement aptes à être absorbées.

(2) La pepsine extraite du Carica papaya, arbre des Moluques et des Antilles, porte le nom spécial de papaïne.

(3) Comparez les notions qui précèdent avec celles qui sont exposées dans le Cours de Zoologie, Anatomie et Physiologie animales, Chap. VII. La substance vivante et ses propriètés, p. 187. 
Siège et conditions de l'assimilation.

Le siège de cette fonction est nettement indiqué; il se trouve dans les feuilles, qui contiennent de la chlorophylle. En outre, cette fonction est caractérisée par une désoxydation énergique, dont on trouve une double preuve dans les deux faits suivants : d'une part, l'activité des cellules à chlorophylle produit un dégagement considérable d'oxygène, et d'autre part, tandis que les aliments puisés au dehors sont extrêmement riches en oxygène, les tissus mêmes des plantes en sont fort pauvres.

Nous savons enfin que les phénomènes d'assimilation ne s'accomplissent que sous l'influence directe des rayons solaires.

Ajoutons, toutefois, qu'une plante renfermée dans un lieu obscur continue à croître; qu'une graine, un tubercule de pomme de terre, placés dans l'endroit le plus sombre, germent, poussent une tige, des feuilles, parfois même des fleurs. C'est qu'il intervient une autre série d'opérations qui s'exercent sur les substances déjà assimilées, mais non employées jusqu'alors à la nutrition de la plante. Ces phénomènes s'accomplissent d'ailleur's également dans les végétaux placés dans les conditions normales. Ces substances, préalablement assimilées dans les cellules chlorophylliennes, peuvent subir des métamorphoses, des changements chimiques plus ou moins complexes, qui les rendent aptes à servir à la nutrition de tels ou tels organes. Ainsi, c'est en vertu d'une opération de ce genre que l'amidon renfermé dans un tubercule de pomme de terre, même placé dans l'obscurité, est employé à la nutrition et au développement des jeunes tiges qui en partent; c'est pourquoi, jusqu'à ce que la réserve nutritive accumulée dans le tubercule soit épuisée, la plante continuera à s'accroître, bien qu'il lui soit impossible de faire des assimilations nouvelles aux dépens de matériaux étranger's, par suite de l'absence de feuilles vertes et d'exposition à la lumière. Mais lorsque cette provision alimentaire sera épuisée, la plante s'arrêtera forcément dans sa croissance et mourra bientôt.

Réserves nutrutives. - L'intervalle qui existe entre l'as- 
similation des substances alimentaires et l'emploi effectif de ces mêmes matières dans la nutrition de la plante est très variable. Ainsi, tandis que fort souvent ces deux ordres d'opérations se font conjointement ou se succèdent immédiatement l'une l'autre, il existe un grand nombre de cas où les choses se passent autrement, et alors les substances assimilées se rendent des feuilles, où elles ont été élaborées, dans certains organes qui varient d'une plante à l'autre. C'est ainsi, par exemple, qu'après la première période de végétation des plantes bisannuelles, on voit que certains organes sont énormément gonflés par les substances nutritives qui s'y accumulent et qui ne seront employées qu'au début de la période végélative suivante. Les diverses espèces ou variétés de Choux nous offrent de ces faits des exemples bien connus : dans le Chou-navet, c'est la racine qui sert de réservoir; dans le Chou-cabus, c'est le bourgeon terminal qui prend un développement énorme, et on dit alors que le Chou est pommé; ce rôle est rempli par les bourgeons latéraux dans le Chou de Bruxelles, enfin par l'inflorescence dans le Chou-fleur. Toutes ces parties gorgées de suc sont fort tendres, savoureuses et nutritives; aussi l'homme les emploie-t-il alors à sa nourriture.

Dans d'autres plantes cette accumulation de sucs se fait dans les tiges souterraines (rhizomes, tubercules), ou bien dans les bulbes et les oignons, dont les écailles se gonflent énormément. Enfin, toutes les graines renferment avec elles une certaine provision de matière nutritive qui servira au premier développement de la jeune plante; ce qu'on appelle leur albumen n'est pas autre chose qu'une réserve alimentaire dont le rôle est de pourvoir à la nutrition de l'embryon, renfermé avec lui dans les enveloppes de la semence.

Classification des substances assimilées. - Au point de vue de leur nature chimique et du rôle qu'elles jouent dans l'organisme végétal, les substances assimilées par la plante peuvent se répartir en trois groupes, à savoir : les 
substances plastiques azotées, telles que la fibrine, l'albumine, la caséine, destinées surtout à l'accroissement du protoplasma, c'est-à-dire, de la substance active par excellence de l'organisme végétal; les substances plastiques non azotées, telles que l'amidon, l'inuline, le sucre, la graisse, etc., destinées, d'une part, à l'accroissement de la paroi des cellules, et d'autre part, à fournir les aliments des combustions intraorganiques et par là même subvenir aux dépenses d'acide carbonique et de vapeur d'eau.

A ces deux groupes essenticls, il faut en joindre un troisième, pour un grand nombre de substances dont le rôle est fort incertain, ou qui même paraissent inutiles à l'organisme, et demeurent sans emploi ; tels sont le tannin, les matières colorantes rouges ou autres (mais non la matière verte ou chlorophylle), les huiles essentielles des glandes, le caoutchouc renfermé dans certains latex, la résine, nombre d'acides, la plupart des alcaloïdes, etc.

Remarque. - L'étude précèdente des phénomènes offerts par la nutrition des végétaux ne peut manquer d'attirer l'attention sur la remarquable analogie qui existe à ce point de vue entre les plantes et les animaux, soit que l'on considère les procédés mis en œuvre, soit que l'on ait égard à la nature des résultats.

Mais une chose doit frapper aussi, c'est qu'à part quelques exceptions, telles que celles que nous ont montrées les plantes carnivores et celles que nous offre la nombreuse classe des Champignons, qui se nourrissent directement de substances organiques, la Plante n'emprunte au milieu extérieur que des substances minérales, qu'elle transforme ensuite en substances organiques, contrairement à l'A nimal, qui emprunte aux végétaux des substances organiques tout élaborées et qui ne saurait exister si celles-ci venaient à lui manquer. 


\section{CHAPITRE VIII}

\section{ORGANES DE REPRODUGTION}

\section{La Fleur.}

Composition d'une fleur. - Inflorescences. - 10 Calice et Corolle : description et structure ; $2^{\circ}$ Étamines et Pollen : description, structure et développement; $3^{\circ}$ Pistil ou Gynécée ; 0vaire, Ovules, Style, Stigmate : description, structure.

Composition d'une fleur. - Une fleur complète, une Rose, un OEillet, par exemple, offre en allant de sa partie

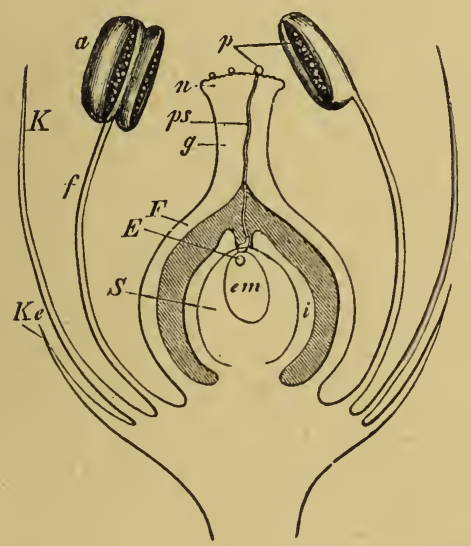

Fig. 91. - Dessin schématique d'une fleur hermaphrodite coupéc suivant sa longueur, pour montrer la situation relative des parties; $K e$, calice; $K$, corolle; $a f$, étamine; $F$, ovaire ; $S$, ovule; $E$, embryon. extérieure à son centre, des organes d'aspect et de structure différents, disposés en zones ou verticilles concentriques; ce sont le calice, la corolle, l'androcée et le pistil ou gynécée (fig. 91).

Le premier est formé par les sépales, le second par les pétales, le troisième par les étamines, le quatrième par les carpelles.

Toutes ces parties ont un rôle à jouer, mais d'importance variable, dans la reproduction de la plante. Le calice et la corolle rie sont, en effet, que des parties accessoires, les enveloppes protectrices des 
organes internes plus délicats; aussi les réunit-on souvent sous le nom commun de périanthe (1). Leurs couleurs brillantes et leur parfum attirent les insectes qui, comme on le verra bientôt, jouent souvent dans la fécondation des plantes un rôle considérable. Tandis que le calice ou la corolle ou même l'un et l'autre peuvent manquer, les deux autres sortes d'organes ont une existence constante, car eux seuls sont essentiels à la reproduction de la plante. Ils ne sont cependant pas toujours réunis sur la même fleur : celle qui ne porte que l'un ou l'autre de ces organes est dite unisexuée et appelée fleur mâle ou fleur femelle, selon qu'elle offre les étamines ou le pistil; quand les deux organes se trouvent réunis sur une même fleur, on donne à celle-ci le nom d'hermaphrodite.

La fleur peut être plus compliquée qu'il n'a été dit plus haut, par suite de la présence d'organes accessoires, notamment de bractées ou feuilles modifiées, qui forment à la fleur une enveloppe extérieure, désignée, suivant les cas, sous les noms d'involucre, de spathe, etc.

Avant d'aborder l'étude des caractères que présentent les différents organes qui viennnent d'être énumérés, il sera bon de donner quelques indications au sujet de la disposition des fleurs sur la tige.

Inflorescences. - la disposition des fleurs sur la tige et les rameaux, ou l'inflorescence, est soumise à certaines règles qui vont être brièvement exposées.

Les fleurs sont axillaires ou terminales.

Dans le premier cas, elles partent de l'aisselle d'une bractée, de sorte que le rameau qui les porte n'étant pas arrêté dans sa croissance, peul en produire un nombre illimité, d'où le nom d'indéfinies donné à ces inflores. cences.

Dans le second cas, une fleur terminant un rameau, celui-ci est, du même coup, arrêté dans sa croissance et l'inflorescence est dite définie ou terminale.

(1) IIspi, autour, $\stackrel{\alpha}{\vee} 00 \varsigma$, fleur. 
Les fleurs axillaires offrent les mêmes variétés de posilion que nous ont présentées les feuilles, c'est-à-dire, qu'elles peuvent être alternes, opposées, verticillées, être sessiles ou pédonculées, solitaires ou groupées et alors géminées, ternées, fasciculées, suivant qu'on en voit 1, 2, 3, ou tout un bouquet partir du même point.

Principales inflorescences indéfinies. - 10 Fleurs sessiles portées directement sur l'axe primaire. Celui-ci

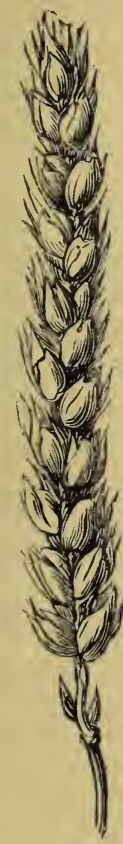

n'offre pas alors de ramifications ou axes secondaires.

A. Épi. Fleurs disposées tout autour de l'axe, de façon à former une inflorescence allangée, cylindrique (Plantain). L'épi peut-être composé (Blé).

$B$. Chaton. Réunion de fleurs" unisexuées, soit mâles soit femelles, disposées en épi; l'axe qui les porte est articulé à sa base et se détache de lui-même à un moment donné (Noyer, Coudrier, Saule).

C. Spadice. Épi dont l'axe est charnu et garni de fleurs unisexuées, le lout recouvert d'une spathe ou enveloppe membraneuse. Il est spécial aux Monocotylédones (Arum ou Pied-de-veau).

D. Cône. Sorte de chaton non caduc, dont les bractées, à l'aisselle desquelles se développent les fleurs femelles, sont de grande dimension et souvent li-.

Fig. 92. - Épi composé gneuses (Pin, Sapin, etc.), d'où le nom (Blé). de Conifères donné aux arbres verts dont cette disposition caractérise les inflorescences.

E. Capitule. Fleurs nombreuses, serrées les unes contre les autres, de façon à former une tête arrondie, groupées sur l'axe floral très raccourci et disposé en une large surface ou réceptacle (Ghardon, Grand-Soleil). L'ensemble est entouré de braclées nombreuses, stériles, c'est-à-dire, 
it l'aisselle desquelles il n'y a pas de fleurs, et constituant ce qu'on appelle un involucre. Quant aux bractées fertiles, qui accompagnent chacune des fleurs du capitule, elles sont réduites à l'état de soies délicates.

F. Sycone. Fleurs unisexuées disposées à la surface d'un réceptacle plan ou concave et plus ou moins clos, qui se charge de sucs et pent devenir comestible (Figue).

$2^{\circ}$ Fleurs pédonculées portées par des axes secondaires ou tertiaires.

G. Grappe. - C'est une sorte d'épi dont l'axe principal porte des axes secondaires terminés chacun par une fleur (Cassis, Groseiller à petits fruits). La grappe est parfois composée, par suite de la ramification des axes secondaires (Vigne); elle porte alors le nom de panicule, expression mauvaise, car elle a été encore employée pour d'autres inflorescences. Il peut se faire que les ramifications situées vers le milieu de l'inflorescence étant les plus longues, celle-ci ait une forme ovoïde allongée ; c'est ce qu'on appelle un thyrse (Lilas).

H. Corymbe. C'est encore une sorte d'épi, dont l'axe principal porte des axes secondaires d'autant plus longs qu'ils sont plus inférieurs; de la sorte, les fleurs qui les terminent arrivent toutes à la même hauteur. Le corymbe peut être simple (Poirier), ou composé, par suite de la ramification des axes secondaires (Sorbier).

I. Ombelle. Du sommet de l'axe primairepartent les axes secondaires qui, de là, vont en divergeant. L'ombelle peut être simple (Primevère, Butomus umbellatus), ou composée, chaque axe secondaire portant à son sommet un certain nombre d'axes tertiaires el terminés chacun par une fleur pour former une ombellule. Cette inflorescence caractérise la famille des Ombellifères (Carotte, Ciguë).

L'ombelle et les ombellules sont pourvues chacune d'une couronne de bractées formant un involucre à la première, des involucelles aux secondes.

INFLoRescence définie. - Il n'y en a qu'une scule, la cyme. Voici en quoi elle consiste : un rameau floral se ter- 
mine par une fleur, et dès lors il ne peut plus s'allonger; mais un peu au-dessous de cette fleur se trouvent deux feuilles ou bractées, de l'aisselle de chacune desquelles naît un axe secondaire également terminé par une fleur; de chacun de ces axes secondaires, au niveau de deux feuilles opposées, naissent de même des axes tertiaires; ainsi de suite. Il en résulte une inflorescence très régulière, et dont les fleurs apparaissent deux par deux. G'est ce qu'on appelle une cyme dichotome ou bipare (Petite Centaurée, Stellaire). Si, au lieu de deux feuilles opposées, les feuilles ou bractées axillantes forment un verticille de trois, la cyme est trichotome ou tripare, les générations de fleurs se montrant alors toujours par trois.

Mais il peut se faire que sur les deux ou trois axes d'une même génération un seul se développe, les autres avortant; la cyme est dite alors monotome ou unipare (Silene gallica).

Cette disposition conduit à une autre variété de cyme, appelée scorpioïde parce qu'elle est contournée en crosse ou queue de scorpion; toutes les fleurs se trouvent du côté convexe du support commun par suite du développement d'axes successifs d'un seul côté (Héliotrope, Myosotis). Dans cette inflorescence, il semblerait, à première vue, que c'est l'axe primaire qui se continue dans toute la longueur de celle-ci; mais il n'en est rien, elle résulte, en réalité, de la formation d'axes successifs, dont chacun usurpant à son tour la direction de l'axe précédent se termine par une fleur.

Telle est encore la cyme héliçö̈de, dont les fleurs et les bractées sont disposées sur l'axe suivant une spirale.

INFLORESCENCES Mixtes. - Enfin, les inflorescences indéfinies et définies peuvent se combiner entre elles pour former des inflorescences mixtes, des grappes de cymes, par exemple, comme dans beaucoup de Silénées.

\section{$1^{\circ}$ Calice et Corolle.}

Caractères du Calice.-Le calice se compose d'un nombre variable de sépales, lesquels peuvent rester libres, 
distincts entre eux, calice dialysépale ou polysépale, ou bien être soudés les uns aux autres, calice gamosépale ou monosépale (fig. 93, A). Dans ce dernier cas, la soudure peut être totale ou plus ou moins étendue. La partie inférieure rétrécie forme le tube du calice, la partie supérieure étalée, le limbe; la limite de séparation des deux régions est la gorge.

Le calice est régulier, quand il est formé de sépales égaux symétriquement disposés; irrégulier, dans le cas contraire.

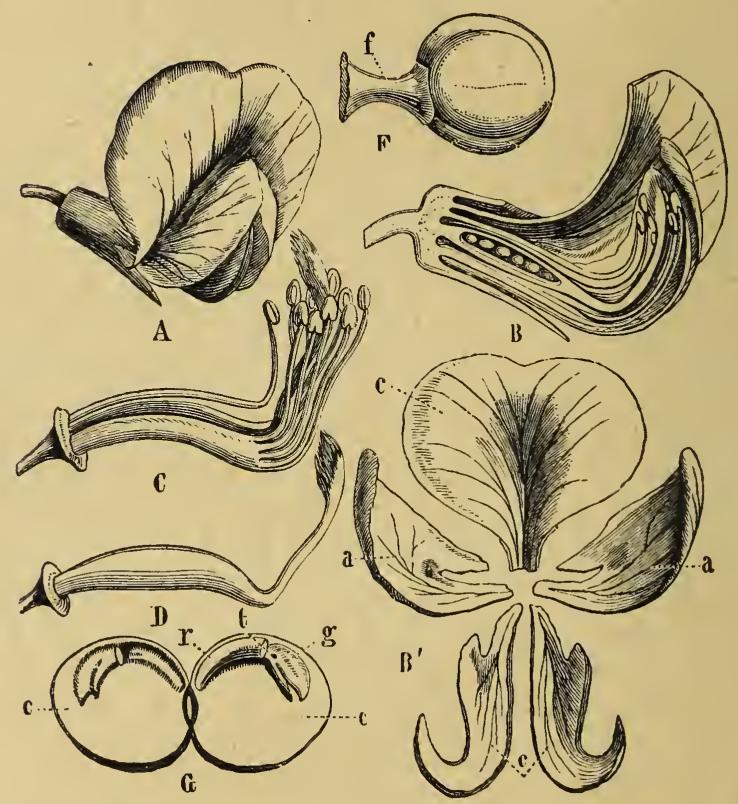

Fig. 93. - Organes floraux d'une Légumineuse (Pois); $A$, ensemble de la fleur; $B$, la même coupće en long; $B$ ', les parties de la corolle séparées; $C$, ensemble du pistil et des étamines toutes soudées entre elles, sauf une; $D$, le pistil isolé; $F$, une graine avec le funicule $(f) ; G$, graine coupée en deux; $c, c$ les cotylédons; $r$, la radicule; $\ell$, la tigelle; $g$, la gemmule.

Caractères de la Corolle. - Comme le calice, la corolle se compose d'un nombre variable de pièces et peut être dialypétale ou polypétale, c'est-à-dire, à pétales distincts, ou bien gamopétale ou monopétale, ou à pétales soudés. Dans l'un et l'autre cas, elle peut être régulière ou irrégulière. 
Les formes principales de corolle polypétale régulière sont désignées sous les noms de cruciforme (Ghoux, Navets), rosacée (Rosier, Pommier), caryophyllée (OEillet); et celle des polypétales irrégulières, sous celui de papilionacée (Légumineuses).

Dans les corolles polypétales, chaque pétale offre à considérer une partie inférieure étroite, l'onglet, et une partie supérieure dilatée, la lame. (Voy. fig. 93, B'.)

Les corolles gamopétales se composent de trois parties, le tube, la gorge et le limbe, comme on l'a vu pour le calice gamopétal.

Les principales formes de corolles gamopétales régulières sont appelées campanulée (Campanule), infundibuiiforme, (Tabac), hypocratériforme (Lilas, Jasmin), rotacée (Pomme de terre), urcéolée (Bruyère).

Parmi les corolles gamopétales irrégulières, les plus importantes sont la bilabiée (Labiées, Verveine) el la personnée (Gueule de Lion).

Structure du Calice et de la Corolle. - Les sépales, Calice. dont la réunion compose le calice, offrent une très grande analogie de structure avec la feuille, de laquelle, comme on le verra un peu plus loin, ils dérivent. On y trouve donc, entre deux couches épidermiques pourvues de stomates, un parenchyme, le plus souvent coloré en vert, au milieu duquel se trouvent de rares faisceaux fibro-vasculaires, dont le principal occupe la ligne médiane de chaque pétale et d'où partent des ramifications formées seulement de quelques trachées.

Les pétales, qui forment par leur réunion la corolle, sont composés de même d'un parenchyme compris entre deux épidermes pourvus de stomates peu nombreux. L'épiderme intérieur a souvent un aspect velouté, dans la Pensée, par exemple, ce qui tient à ce que ses cellules forment des saillies en manière de petits cônes ou papilles. En outre, les pétales sont parcourus par de fines nervures ramifićes comme celles des feuilles, mais à peu près réduites à quelques trachées.

Corolle. 
Coloration et parfum des pétales.

Le parenchyme est formé de cellules molles, remplies de liquide, contenant souvent des gaz et colorées de nuances très varićes dues à des causes diverses, suivant les cas. Ainsi les fleurs qui sont d'un blanc de lait, comme celles du Lis, doivent leur blancheur à une grande quantité de gaz renfermés dans les cellules; aussi, dès que l'on vient à les en chasser par la pression ou au moyen de la machine pneumatique, on voit la fleur perdre son éclatante blancheur. D'autrefois, la couleur est due à des liquides colorés; telle est la teinte bleue de la Campanule, dont les principes colorants sont logés dans les deux épidermes; de même, aussi, les coulenrs roses ou rouge clair, violettes, sont également dues le plus souvent à des liquides. Enfin, le jaune, le rouge foncé, le brun sont plutôt le fait de granulations colorées en suspension dans un liquide ou dans le protoplasma cellulaire.

D'ailleurs, il peut se faire que la couleur des pétales dépende à la fois de plusieurs causes, telles que le mélange ou la superposition de cellules renfermant des liquides et des grairulations solides de couleurs différentes; c'est le cas de la Petite Capucine, si vivement colorée en rouge, dans laquelle on voit plusieurs couches de cellules superposées les unes à suc cellulaire rouge, d'autres avec des granulations jaunes.

Les pétales sont également le siège le plus ordinaire des parfums qu'exhalent les fleurs d'un si grand nombre de plantes et qui consistent le plus souvent en essences, logées soit dans les cellules mêmes du parenchyme, soit dans des organes spéciaux, petits réservoirs que l'on peut parfois voir par transparence, comme cela est facile dans les pétales du Citronnier et de l'Oranger.

20 Étamines et Pollen.

Garactères des Étamines. - Une étamine complète se compose de trois parties : le filet, sorte de petit support ou 
pédicule (fig. $91, f)$; l'anthère $(a)$, partie principale de l'étamine, car c'est elle qui renferme le pollen ou poussière fécondante de la fleur ; enfin le connectif, qui fait suite au filet et qui est interposé entre les loges de l'anthère. Le filet peut manquer, et l'étamine est dite sessile.

Les étamines offrent un grand nombre de particularités dont on tient compte dans la description et la classification des espèces végétales. Elles vont être brièvement indiquées.

A. Nombre absolu. Très variable, puisqu'il va de 1 à plu sieurs centaines, on l'indique par des noms particulier's; ainsi on appelle fleur monandre celle qui n'a qu'une étamine (Valériane rouge, Balisier, fig. 94); puis, jusqu'à 10 étamines, on a successivement les fleurs diandre (Véronique), triandre (Blé, Iris), tétrandre (Caille-lait), pentandre (Belladone), hexandre (Lis), heptandre (Marronnier d'Inde), octandre (Bruyère), ennéandre (Laurier), décandre (OEillet). $\mathrm{Au}$ delà de dix, on appelle dodécandres celles qui ont de 12 à 20 étamines; passé ce nombre, les fleurs sont dites polyandres (Pavot, Chélidoine, fig. 95, I, IV).

B. Nombre relatif à celui des pièces de la corolle. Si les étamines sont en même nombre que les pétales, la fleur est isostémone; dans le cas contraire, anisostémone; on la dit enfin méiostémone ou polystémone, selon que les étamines sont moins nombreuses ou plus nombreuses que les pétales, et diplostémone, quand elles sont en nombre double de ceux-ci.

C. Variétés de longueur. Souvent toutes de même longueur, les étamines sont parfois les unes plus longues, les autres plus courtes. Ainsi dans l'Oseille, cinq étamines sont très petites et cinq beaucoup plus longues; on appelle didynames les étamines, qui, au nombre de quatre dans une même fleur, ont deux d'entre elles plus grandes (Labiées), tétradynames, celles qui étant au nombre de six, ont quatre d'entre elles plus grandes (Crucifères).

D. Variétés de situation et d'insertion. Ordinairement alternes avec les pièces de la corolle, elles leur sont assez souvent opposées. (Voy. Chap. Ix. Plan de la fleur.) Ordinairement insérées sur le réceptacle floral, entre la corolle 
et le gynécée, clles sont parfois portées par les pièces de la corolle, comme c'est la règle dans les cas de corolle gamopétale, d'autres fois par les carpelles (Orchidées, Aristoloches); et l'on donne le nom de colonne ou gynostème à l'organe qui résulte de la soudure de ces deux sortes d'organes.

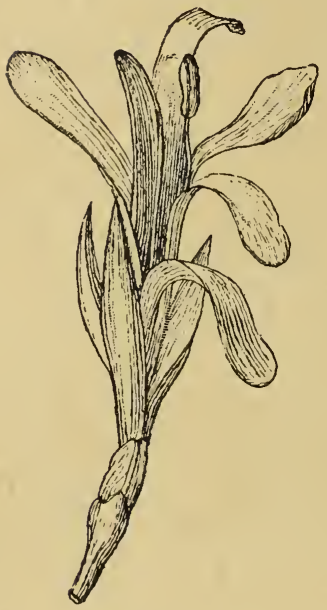

Fig. 94.

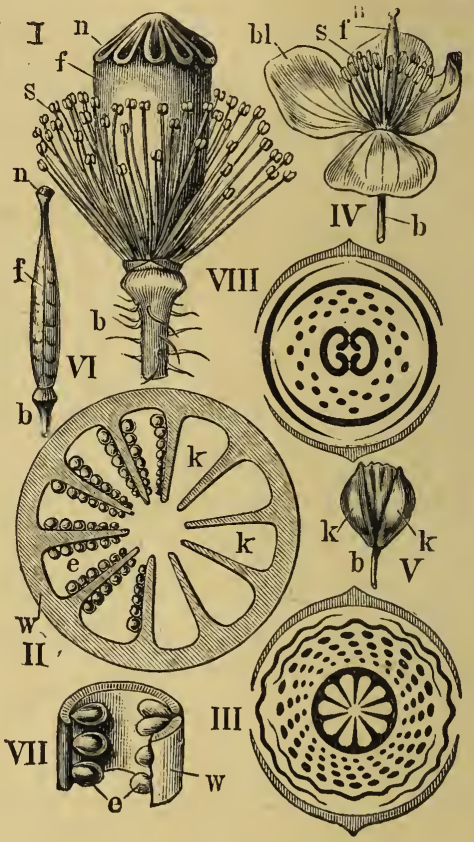

Fig. 95 .

Fig. 94. - Fleur monandre (Balisier).

Fig. 95. - I à III, Organes floraux du Pavot (Papaver rhoas); I, androcéc et pistil (gross. 2); II, coupe transversale de l'ovaire (gross. 4); III, diagramme de la fleur; IV a VIII, organes floraux de la Grande Éclaire (Chelidonium majus); IV, ensemble de la fleur; V, fleur en bouton; VI, fruit ou silique (grand. nat.); VII, segment de la silique très grossi et montrant les ovules; VIII, diagramme de la fleur. - Les mêmes lettres désignent les mêmes objets dans les difrérentes figures; $b$, pédoncule floral; $k$ (de la fig. V), calice; $b l$, corolle; $s$, étamines; $f$, ovaire; $n$, stigmate; $w$, pa roi de l'ovaire; $\mathrm{k}$ (de la fig. II), cloisons de l'ovaire

Relativement à l'ovaire, les étamines peuvent être insérées autour, au-dessus ou au-dessous de lui, de sorte qu'il y a lieu de distinguer des étamines périgynes, épigynes el 
hypogynes. Ces différents modes d'insertion caractérisent assez exactement un certain nombre de familles naturelles.

CARACTÈRES OFFERTS PAR LE FILET. - La forme extérieure du filet lui vaut les épithètes de capillaire, eylindrique, pétaloïde, selon qu'il est grêle, plus épais et arrondi, étalé en forme de pétale. (Voyez fig. 97 et fig. 98.)

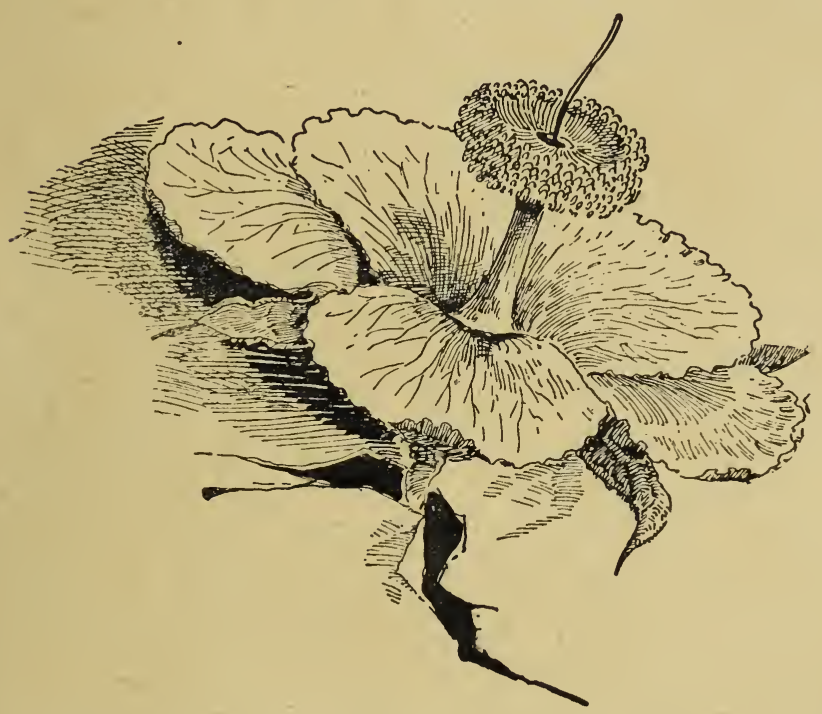

Fig. 96. - Fleur de Baobab montrant les filets staminaux formant par leur soudure un androphore (étamines monadelphes), surmonté par le style du gynécée.

Les filets staminaux peuvent se souder entre eux dans une étendue variable et constituent ce qu'on appelle un androphore; s'ils sont tous réunis de la sorte, les étamines sont dites monadelphes (Mauve, Rose trémière, Baobab, fig. 96) ; si elles forment deux groupes séparés on les appelle diadelphes (Fumeterre, Haricot, fig. 93, C); si elles en constituent plusieurs, comme dans le Ricin, elles sont polyadelphes. 
Forme. Caractères offerts par L'Anthère. - Suivant la forme extérieure de l'anthère, on la dit ovoïde, globuleuse, réniforme, linéaire, cordiforme, expressions qui se comprennent d'elles-mêmes.

Loges. $\quad$ Elle est creusée ordinairement de deux loges et dite alor's biloculaire; exceptionnellement elle n'en possède qu'une (Malvacées), ou quatre (Butome ombellé), et inérite alor's suivant le cas, le nom d'uniloculaire ou de quadriloculaire.

Insertion.

Ordinairement parallèles au filet, les loges de l'anthère s'en écartent parfois à l'une où l'autre extrémité et sont appelées divergentes, et horizontales quand elles s'en écartent

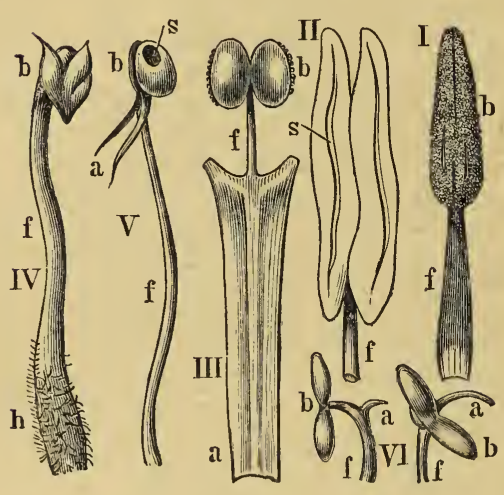

Fig. 97 .

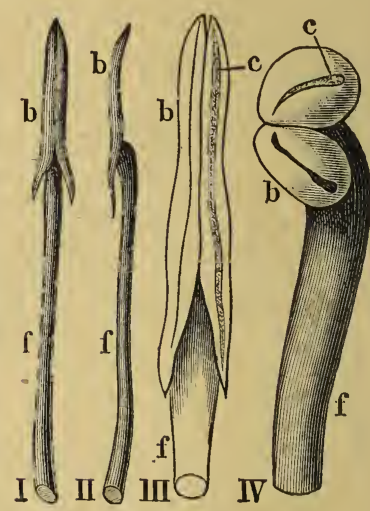

Fig. 98 .

Fig. 97.-Différentes formes d'ćtamines ; I, Tulipe (Tulipa gesneriana) (gros. 2); II, Graminéc (Phalaris arundinacea) (gros. 4); IlI, Deulsia glabra (gros. 4); IV, Orobanche (Orobanche rubens) (gros. 3); V, Bruyère (Erica tetralix) (gros. 3); VI, Pruncllier (Prunella vulgaris) (gros. 5). - $f$, filet; $b$, anthère; $s$, déhiscenec ; $a$, appendice; $h$, poils.

Fig. 98. - Étamines montrant leur mode de déhiscence; I à III, étamines de Glaïeul (les deux premières de grand. natur., la troisième gros. 3 fois); $f$, filet, $b$, anthère; $c$, fente ou ligne de déhiscenec de l'anthère. - IV, Étamine très grossie de la Gucule de Lion (Antirrhinum majus); $f$, filet; $b$, anthère; $c$, ligne de déhiscence.

au point de lui devenir perpendiculaires. Si l'anthère est mobile sur le filet au lieu de lui être solidement fixée, comme c'est le cas ordinaire, on la dit oscillante ou versatile; enfin on l'appelle basifixe, quand elle est altachéc 
par sa base, médifixe, quand elle s'insère par sa partie moyenne, apicifice, lorsque, attachée seulement par son sommet, elle y est comme suspendue.

L'une des faces de l'anthère offre, à un moment donné,

Déhisecnce, de petites ouvertures pour la déhiscence ou anthèse, c'est-à-dire, la sortie du pollen; si la face qui porte ces orifices est tournée vers le centre de la fleur, l'étamine est introrse; dans le cas contraire, elle est extrorse.

La déhiscence se fait le plus souvent par une fente longitudinale placée sur chaque loge de l'anthère, mais parfois au moyen d'un trou ou pore (déhiscence poricide), ou bien au moyen de valves ou panneaux, au nombre de 2 ou 4 par anthère (déhiscence valvaire).

Les anthères sont parfois réunies les unes aux autres de façon à former un tube par lequel passe le style du gynécée, disposition caractérisée par l'expression de synanthérée el qui a valu son nom à une nombreuse famille, celle des Synanthérées ou Composées.

Caractères du Pollen. - Le pollen (fig. 99) est constitué Forme. par des grains d'une finesse extrême, qui n'ont pas plus de vingt millièmes de millimètre dans la Betterave et même dix millièmes dans le Myosotis, mais qui dans la Belle-de-Nuit atteignent jusqu’à un septième de millimètre de diamètre. Ces grains sont ordinairement arrondis, parfois polyédriques, tantôt lisses à leur surface et tantôt marqués de dessins variés, de ponctuations, de granulations, de petits prolongements en forme de piquants, de plis, de trous parfois fermés par' un petit opercule; ces orifices, dont le nombre varic de 2 ou 3 jusqu'à 200, permettent à un moment donné la sortie de la substance intérieure. (Voy. chap. x, Fécondation.)

En général, les grains de pollen restent isolés, libres entre eux, et le pollen est dit pulvérulent. Il n'en est pas toujours ainsi; dans certaines espèces, ils sont accolés, agglutinés entre eux en une masse solide qui a la forme même de la loge de l'anthère où le pollen s'est développé (Orchidées). Parmi les Dicotylédones, les Asclépiadacées offrent également des masses polliniques (tig. $101 \mathrm{G}$ ).

Soudure. 
La structure des yrains de pollen agglutinés de la sorte est plus simple que celle des autres; ils sont dépourvus de plis et de perforations et ne possèdent, comme on le verra bientôt, qu'une seule enveloppe.

Filet.

Structure de l'Étamine et du Pollen. - Le filet offre une grande analogie de struc-

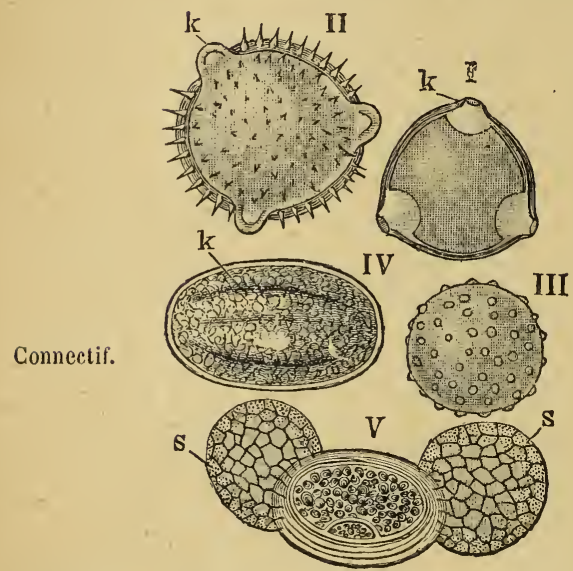

Anthère et pollen.
Fig. 99. - Difrérentes formes de grains de pollen grossis 300 fois; I, du Noisetier (Corylus avellana); II, du Tussilage (Tussilago farfara); III, du Cabaret (Asarum europoum); IV, d'Hépatique (Hépatica triloba); V, du Pin (Pinus sylvestris). - $k$, pores; $s$, vésicules pleines d'air. ture avec le pétiolè de la feuille; comme lui, il présente des éléments fibro-vasculaires entourés de cellules; le tout est recouvert par un épiderme pouvu de stomates.

Le connectif se compose d'éléments fibro-vasculaires, continuation de ceux du filet, et aussi, de cellules molles et allon-. gées.

L'anthère, pour être connue, demande qu'on en suive toute l'évolution, depuis sa première apparition, jusqu'au moment où les loges qui vont s'y creuser s'ouvrent pour laisser échapper le pollen.

Développenent de l'anthère et du pollen
G'est d'abord sous forme d'un mamelon que cette partie de l'étamine apparaît (fig. 100); ce mamelon s'élargit à droite et à gauche et se creuse d'un sillon médian longitudinal, premier indice de la formation des deux lobes dont l'anthère se composera; puis, chacun de ces lobes est divisé à son tour par un petit sillon marquant la ligne de séparation de deux logettes, dont chacun d'eux va se creuser(fig.100,A, $G$ ).

En même temps que ces phénomènes se passent ù l'extérieur de l'anthère, voyons ce qui se produit au dedans. 
Le tissu intérieur, qui jusqu'alors était formé de cellules toutes semblables, éprouve de notables changements.

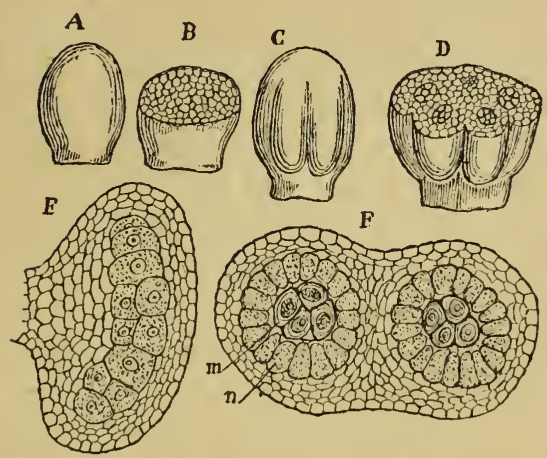

Fig. 100. - Développement de l'anthère et du pollen; $A$, étamine apparaissant sous forme d'un petit mamelon; $B$, coupe transversale de l'étamine montrant un tissu cellulaire homogène; $C$, étamine plus développée avec commencement de délimitation des loges; $D$, sa coupe transversale, montrant le début de la formation des logettes anthériques ảu nombre de quatre (d'après $\mathrm{H}$. Baillon); $F$, coupe transversale d’une anthère à deux loges; $m$, grains de pollen résultant de la division d'une cellule-mère en quatre parties; $n$, cellules destinćes à servir d'aliment aux grains de pollen; $E$, coupe longitudinale montrant les cellules-mères des grains de pollen disposées en file.

Dans chaque endroit où une logette doit se produire on voit une file de cellules superposées (fig. 100, E), qui s'agrandissent considérablement, pour se diviser bientôt en
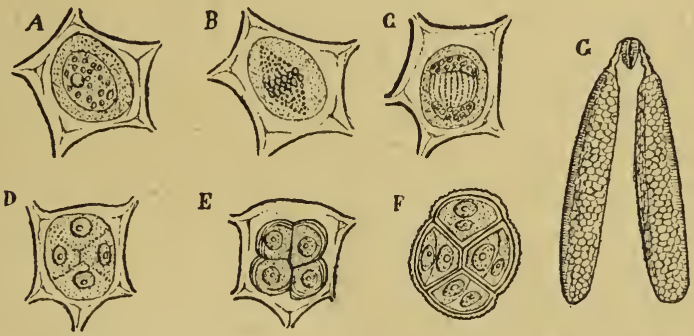

Fig. 101. - Formation des grains de pollen; $A, B, C, D, E$, phases suecessives par lesquelles passe une cellule-mère du pollen pour se diviser en quatre grains; $F$ montre chaque grain de pollen se divisant à son tour en deux parties dont la plus grosse seule doit germer, $c^{*}$ est-à-dire produire le tube pollinique $; G$, masse polliniqne d'une Asclépiadée (Asclepias floribunda); tous les grains de pollen d'une même loge restent agglutinés. 
deux, quatre parties ou plus, suivant les espèces, et aux dépens desquelles se formeront les grains de pollen, d'où leur nom de cellules-mères du pollen ou utricules polliniques. Bientôt, en effet, chaque utricule pollinique se divise en quatre autres cellules qui se partagent à leur tour par quatre pour former autant de grains de pollen, lesquels grossissent et se serrent les uns contre les autres (fig. 101). Réunis d'abord entre eux par une substance gélatineuse intercellulaire, celle-ci disparaît bientôt, et les grains se trouvent isolés; cependant il peut arriver que cette matière persiste, et alors les grains de pollen soit d'une mème utricule, soit de plusieurs, ou même tous ceux d'une même logette restent adhérents de façon à former une seule masse pollinique (fig. 101, G). Les Orchidées, les Asclépiadées offrent des exemples frappants de ce dernier cas.

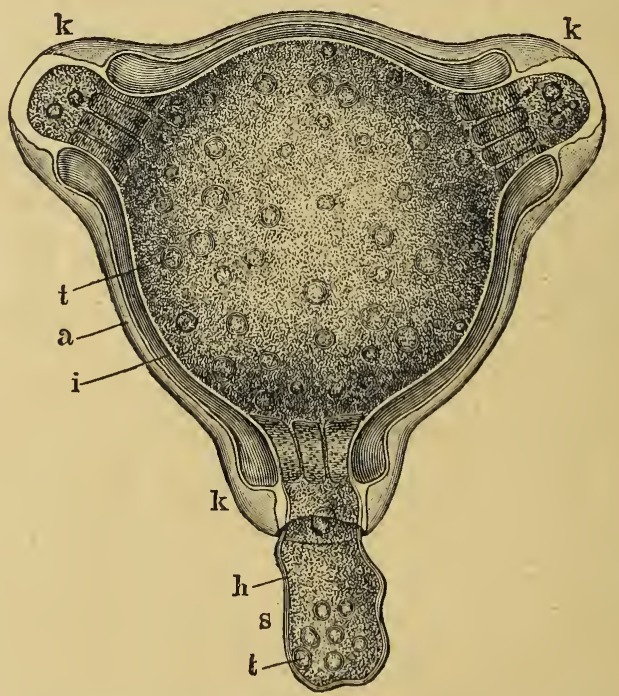

Fig. 102.- Grain de pollen, coupé transversalement et grossi 600 fois, de l'Épilobe (Epilobium angustifolium); $k$, pores ; $a$, exine ; $i$, intine; $s$, boyau pollinique; $h$, son enveloppe; $l$, gouttelettes disséminées dans la masse intéricure ou fovilla. 
cellule; il se compose par conséquent, d'une paroi ou membrane d'enveloppe et d'un contenu.

La membrane est double, c'est-à-dire qu'elle comprend deux enveloppes emboîtées l'une dans l'autre, mais ayant des caractères bien différents (fig. 102) : l'interne est molle, très extensible, élastique, c'est l'intine ou endhyménine; l'externe est résistante, inextensible, pourvue de prolongements ou de dessins variés et seule percée de pores en plus ou moins grand nombre, c'est l'exine ou exhyménine; cetle dernière manque dans certains cas, notamment dans les masses polliniques.

Le contenu du grain de pollen ou la fovilla (1) est composé de protoplasma tenant en suspension différents granules animés de mouvements variés (mouvement brownien), particulièrement des grains d'amidon et aussi des gouttelettes d'huile. Enfin, dans le protoplasma se voit un noyau.

Sous l'influence de la pénétration, par endosmose, d'une certaine quantité de liquide dans l'intérieur du grain de pollen, la fovilla augmente de volume et repousse l'intine, qui passe à travers les pores de l'exine en formant ce qu'on nomme le tube ou boyau pollinique, sur lequel on reviendra au Chapitre de la fécondation des végétaux.

Modification de structure des parois de Lanthère. Tandis que les grains de pollen se forment et grossissent, la paroi de l'anthère, formée de 3 ou 4 couches concentriques de cellules, présente d'importants changements. La couche la plus voisine des utricules polliniques (fig.100,F, $n$ ) se ramollit, devient gélatineuse et finalement se résorbe à mesure que les grains de pollen grossissent.

En dehors de cette zone s'en voit une autre qui va jusqu'à l'épiderme et qui se compose de cellules résistantes, marquées d'épaississements réticulés ou spiralés, et auxquelles on a donné bien à tort le nom de cellules fibreuses (fig. 103 et 104), car nous avons vu dans le premier Chapitre (p. 12), ce qu'il fallait entendre par fibres.

(1) On écrit aussi, et avec plus de raison favilla, mot latin qui signifie fine poussière. 
Enfin, tout à l'extérieur, se voit l'épiderme avec ses stomates (fig. 103, $w$ et 104).

D'après cela, la coupe transversale d'une étamine complètement développée montre donc une couche épidermique, une couche de cellules fibreuses placées sur un, deux,

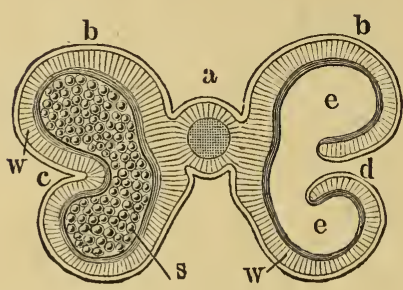

Fig. 103.

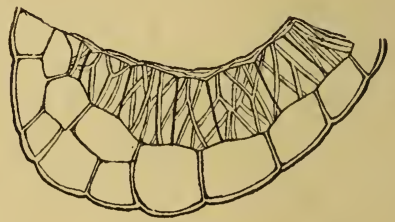

Fig. 104 .

Fig. 103. - Coupe transversale d'une antlì̀re entièrement développée de la Fritillaire (Fritillaria imperialis) (gross. 15); $a$, connectif ; $b, b$, loges de l'anthère; $c$, sillon longitudinal séparant les logettes primitives; $d$, fente de l'anthère au moment de la déhiscence; $e$, cavité dont est creusée l'anthère; $s$, grains de pollen qui la remplissent; $w$, paroi de l'anthère.

Fig. 104. - Portion très grossie de la paroi de l'anthère. En dehors, grandes cellules épidermiques; en dedans, cellules fibreuses.

ou trois rangs, et formant la paroi des logettes, dans lesquelles se trouvent entassés les grains de pollen. Dans la grande majorité des cas, les logettes sont d'abord au nombre de quatre, mais la mince cloison qui sépare les logettes d'un même côté se rompt, et les deux petites cavités communiquent l'une avec l'autre, de sorte que l'anthère n'offre plus en définitive que deux loges et est dite biloculaire.

Ajoutons que la disposition des cellules fibreuses varie avec le mode de déhiscence; ainsi dans les anthères dont la déhiscence se fait par des pores terminaux (Bruyère), elles manquent complètement; très souvent elles sont localisées dans certaines régions, par exemple, de chaque côté de la ligne suivant laquelle se fend chaque loge à la maturité du pollen. Aussi s'accorde-t-on généralement à leur attribuer un rôle prépondérant dans le phénomène de la déhiscence. 
30 Pistil ou Gynécée (1).

Parties constitutives du Pistil. - Le pistil est l'ensemble des carpelles ou organes femelles de la fleur (2).

Un carpelle se compose de trois parties : $1^{\circ}$ l'ovaire, qui renferme les ovules, $2^{\circ}$ le style et $3^{\circ}$ le stigmate.

Ovaire et Placenta. - Tantôt le gynécée ne se compose que d'un carpelle et on dit l'ovaire uniloculaire ou à une seulc loge, et tantôt de plusieurs, lesquels peuvent rester distincts les uns les autres (Pivoine), ou être soudés entre eux dans une étendue variable. Dans ce cas l'ovaire peut
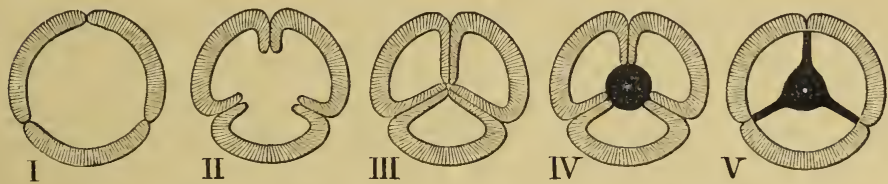

Fig. 105. - Disposition des feuilles carpellaires pour constituer l'ovaire. I, ovaire à une seule loge; II, ovaire avec cloisons incomplètes; III, ovaire divisé en trois loges; IV et V, ovaires divisés en trois loges, en partic ou en totalité au moyen de fausses cloisons.

être encore uniloculaire ou bien multiloculaire. Le plus souvent, en effet, les carpelles qui, comme on le verra bientôt, ne sont que des feuilles repliées sur elles-mêmes, soudent leurs bords l'un à l'autre, de façon à former chacun une cavité distincte, de sorte qu'il y a aulant de loges que de carpelles. Mais d'autres fois, au lieu de se replier en dedans, les feuilles carpellaires voisines se soudent bord à bord pour former une cavité unique.

(1) Pistil, de pistillum, pilon, en raison de sa forme mince et

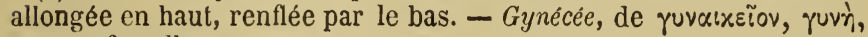
organe femelle.

(2) Carpelle, de xapròs, fruit. 
Dans tous les cas, les ovules sont insérés par l'intermédiaire d'un petit cordon de longueur variable, le funicule

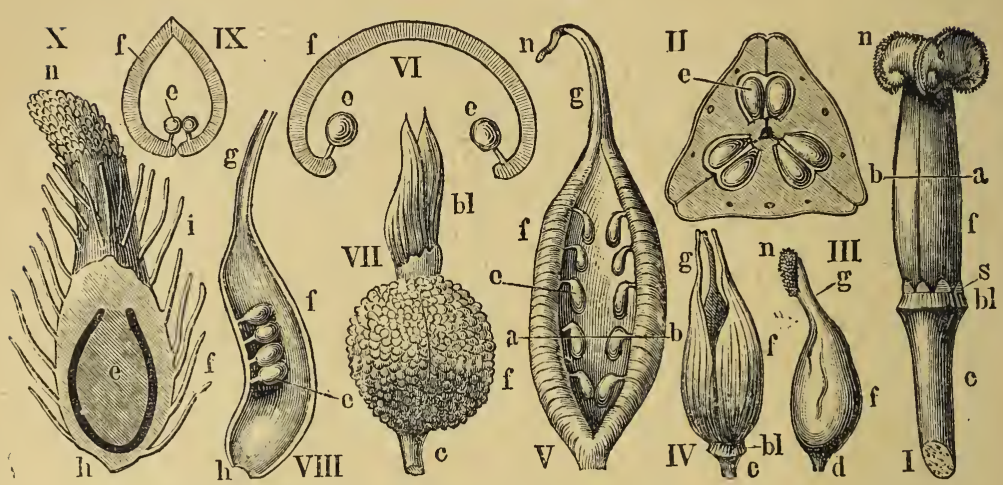

Fis. 106. - Diflérentes sortes d'ovaires montrant l'insertion des ovules. I, Tulipe (Tulipa Gesneriana) (gr. nat.); II, coupe transversale de la même, (gr.4) ; III, Pigamon (Thalictrum aquilegifolium) (gr. 5); IV, Millepertuis (Iyperieum clodes) ; V, le même, montrant ses cloisons incomplètes (gr. 7) ; VI, coupe transversale d'une feuille carpellaire du même; VII, Balisier (Canna indieca) (gr. nat.); VIII, une moitié de l'ovaire de l'Ellébore (Helleborus virillis); IX, coupe transversale du même (gr. 3); X, Hépatique (Hepatica triloba), dont une portion de la paroi a été enlevée (gr. 5 ); $-c$, pédoncule floral; $d$, pédicule de l'ovaire; $b l$, disque floral, lieu d'insertion du périantle; $s$, lieu d'insertion des étamines; $f$, ovaire ; $g$, style $; n$, stigmates $; e$, ovules; $i$, poils; $a b$, lignes sui vant lesquelles sont faites les coupes transversales.

Cloisons vraies ou fausses.

ou podosperme (1), sur un épaississement présenté par le bord des feuilles carpellaires à leur ligne de jonction, et qu'on appelle le placenta ou trophosperme (2).

L'ovaire étant souvent divisé en plusieurs loges, les cloisons qui les séparent sont dites vraies quand elles sont formées par les bords mêmes des feuilles carpellaires repliés vers le centre de la fleur; alors ¿les ovules sont

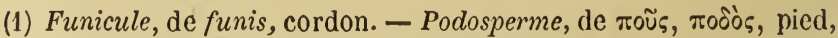
et $\sigma \pi \varepsilon ́ p \mu \alpha$, graine. On dit aussi cordon ombilical.

(2) Placenta, mot emprunté à l'anatomic animale et qui signific à proprement parler gâteau, en raison de la forme arrondic et aplatic de cet organe chez les animaux. - Trophosperme, de tpop h̀, nour-

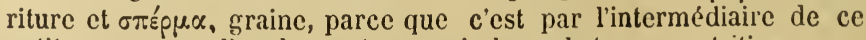
petit organe que l'ovule peut recevoir les substances nutritives. 
insérés seulement dans l'angle qui résulte de cette soudure, et le placenta qui les porte est dit axile (fig. 105, III et $107, \mathrm{I})$; on les appelle fausses, lorsque les carpelles étant
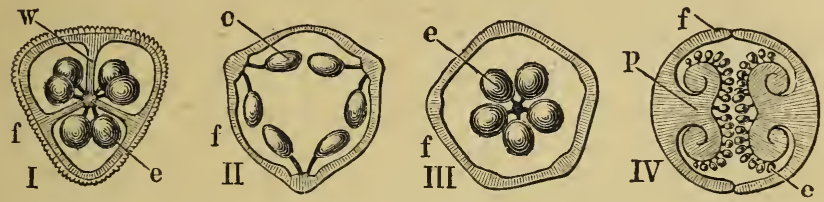

Fig. 107. - Situation des ovules dans l'ovaire; I, Balisier : ovaire à trois loges et placenta axile (gr. nat.); II, Violette : ovaire uniloculaire et placentas pariétaux; III, Primevère : placenta central; IV, Lathrée : placentas développés en fausses cloisons. - $f$, paroi de l'ovaire; $w$, cloisons ovariennes; $p$, placenta ; $e$, ovule.

disposés en une cavité unique, ces cloisons ne sont dues qu'à un développement variable des placentas (fig. 104, IV, V), qui partant de la paroi de l'ovaire, d'où leur nom de placentas pariétaux, s'avancent vers le centre de la cavité (fig. 95, II ; 107, IV); parfois ils ne font qu'une faible saillie dans l'intérieur de l'ovaire (fig. 107, II).

On distingue toujours ces fausses cloisons des premières par ce fait qu'elle sont presque entièrement recouvertes par les ovules et qu'elles alternent avec les styles et stigmates au lieu de leur être opposées.

Les cloisons vraies ou fausses sont dites complètes ou incomplètes, selon qu'elles s'avancent jusqu'au centre de la cavité ovarienne ou au contraire ne constituent qu'une saillie plus ou moins prononcée (fig. 105, II ; 106, V; 107, IV).

Par exception, les placentas peuvent former au centre de l'ovaire uniloculaire une colonne isolée, d'où Jeur nom de placenta central (Primulacées, fig. 107, III).

L'ovaire repose ordinairement par sa base sur le réceptacle; mais parfois il est porté par un support plus ou moins allongé, nommé podogyne (fig. 106, III, $d$ ).

L'ovaire est dit libre ou supère quand il est isolé du périanthe floral, non soudé avec lui, comme dans le Fraisier (fig. 108, B), et au contraire adhérent ou infère quand, situé à un niveau plus inférieur, il est soudé avec le tube du calice comme dans le Pommier $(\boldsymbol{D})$; un état intermédiaire 
est offert par l'ovaire pariétal, dont la base seulement est unie à la face interne du calice, ainsi que le montre le $\operatorname{Rosier}(C)$.
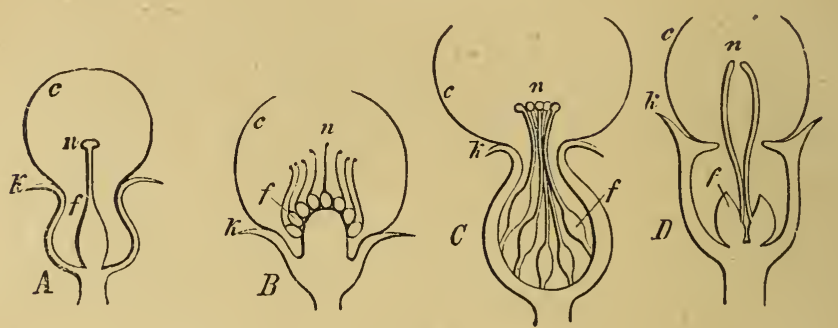

Fig. 108. - Situation de l'ovaire relativement aux autres parties de la fleur; $A$, Amandier; $B$, Fraisier; $C$, Rosier ; $D$, Pommier. $-k$, calice ; $c$, corolle; $f$, ovaire; $n$, stigmate.

Structure De L'Ovaire. - Les feuilles dites carpellaires, qui constituent les parois de l'ovaire, étant dérivées de feuilles repliées sur elles-mêmes etsoudées par leurs bords de façon à former une cavité, comme on le verra bientôt au Chapitre Ix, on comprend que leur structure ne s'éloigne guère de celle de la feuille. En effet, on y voit un épiderme extérieur et un intérieur qui tapisse la cavité carpellaire ; entre les deux existe un parenchyme traversé par des faisceaux fibro-vasculaires venus du pédoncule floral, dont les principaux se voient sur la ligne dorsale du carpelle, et qui se divisent dans son épaisseur pour aller les uns dans le style et les autres jusque dans les placentas et les ovules.

Le petit cordon ou funicule qui rattache chaque ovule au placenta est traversé dans loute sa longueur par des trachées venues de la paroi ovarienne et qui vont jusqu'à l'ovule lui-même où, d'ordinaire, elles s'arrêtent brusquement; mais lorsque ce funicule se prolonge sur le côté de l'ovule de façon à former un raphé, comme on va bientôt le voir, ces vaisseaux suivent le même trajet el se distribuent même dans le tégument extérieur de l'ovule.

Ovule. - L'ovule est un petit corps d'une importance considérable, car c'est à lui qu'échoit le rôle de repro- 
duire la plante, c'est-à-dire de devenir la graine, mais seulement après qu'il a subi l'influence fécondante du pollen. Tandis que dans certains ovaires on n'en rencontre qu'un seul, il en est où l'on en compte un nombre immense.

Il apparaît au bord des feuilles carpellaires, sur la saillie formée par le placenta, auquel il est rattaché par le funicule, quelquefois assez allongé, d'autres fois à peu près nul, de sorte qu'alors l'ovule est sessile. 11 a d'abord la forme d'un petit mamelon, tout entier formé d'un tissu cellulaire homogène. Bientôt à sa base se montre un bourrelet circulaire qui s'étend de plus en plus à la surface du mamelon, et qui finit par le recouvrir entièrement; puis un second bourrelet se montre à l'extérieur du premier et se comporte de même. Ge sont les enveloppes de l'ovule, lesquelles ont reçu les noms, l'extćrieure, de primine, l'intérieure, de secondine, tandis que la petite masse centrale qu'elles recouvrent est le nucelle (1). Les deux enveloppes, par le progrès de leur développement, rejoignent presque leurs bords au-dessus du nucelle, et ne laissent libre qu'un étroit passage; l'orifice de la primine est l'exostome, celui de la secondine l'endostome; ces deux orifices réunis constituent le micropyle (fig. 109).

L'ovule adhère à ses membranes enveloppantes seulcment par sa base, qui a reçu le nom de chalaze, tandis que le point de l'ovule où s'attache le funicule porte le nom de hile.

D'après les rapports dans lesquels se trouvent l'un par rapport à l'autre la chalaze, le hile et le micropyle, on dis- sortes d'ovules. tingue trois sortes d'ovules, à savoir : l'ovule droit ou orthotrope (2), dans lequel le micropyle occupe le point opposé à la chalaze, qui reste alors exactement superposée au hile, de sorte que ces trois parties se trouvent sur une même ligne droite qui traverserail le nucelle dans toute sa longueur

(1) On fait parfois et avec plus de raison nucelle du féminin.

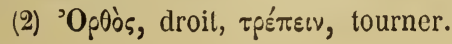

Parties constitutives. 
(Noyer, fig. 109, A) ; l'ovule courbé ou campylotrope (1), dans lequel, par suite d'un développement inégal, il se produit une courbure telle que le micropyle se rapproche du
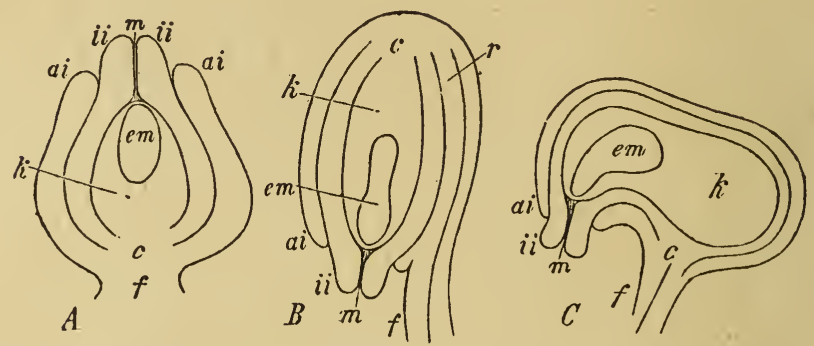

Fig. 109. - Les trois formes d'ovules : $A$, orthotrope; $B$, anatrope; $C$, campylotrope. $-f$, hile; $c$, base de l'ovule ou chalaze; $a i$, la primine; $i$, la secondine; $k$, le nucelle; $m$, le micropyle; $c m$, l'embryon ; $r$, raphé.

hile, dont les rapports avec la chalaze n'ont pas changé; autrement dit, le sommet s'est rapproché de lạ base $(C)$ (Crucifëres, Papilionacées); l'ovule réfléchi ou anatrope (2), enfin, dans lequel le nucelle semble avoir subi un mouvement complet de rotation sur lui-même, de sorte que le micropyle, se rapproche du hile, comme dans le cas précédent, mais en même temps que la chalaze s'éloigne de celui-ci et s'en trouve séparée par toute la longueur du nucelle $(B)$. Celte disposition s'accompagne d'une particularité remarquable, la production d'un raphé(3); elle consiste en ce que le faisceau fibro-vasculaire venu des parois de l'ovaire pour se distribuer à l'ovule en passant par le funicule, au lieu de traverser directement les deux membranes, s'allonge entre elles sur l'un des côtés, en formant une saillie longitudinale qui se termine au point opposé de l'ovule (fig.109, B, r). Cette disposition constante dans l'ovule anatrope ne se rencontre dans aucun autre cas (Renonculacées, Liliacées, ctc.).

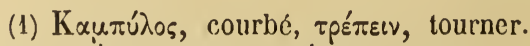

(2) 'Av'̀, en haut et $\tau \rho \rho^{\prime} \pi \varepsilon \iota \nu$, tourner.

(3) 'P $\alpha \varphi \hat{\eta}$, couture. 
Il faut remarquer que la structure de l'ovule peut être plus simple, le tégument étant unique (Gymnospermes, beaucoup de Dicotylédones gamo-

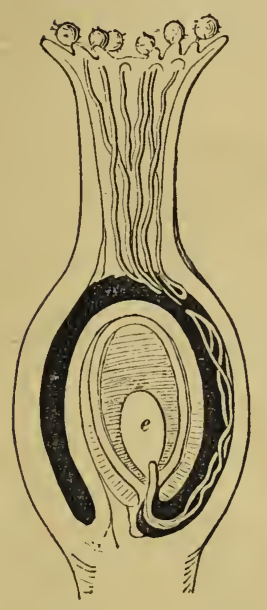

Fig. 110. - Coupe longitudinale a'un ovaire renfermant un ovule anatrope, où l'on voit le trajet que suivent les tubes polliniques pour arriver jusqu'au nucelle $(e)$. pétales et quelques dialypétales, comme les Ombellifères); il peut même manquer tout à fait, de sorte que l'ovule, réduit au nucelle, est $m u$ (Santalacées).

Des changements importants se passent dans l'ovule même avant la fécondation. Le nucelle se creuse la récondation. d'une petite cavité que l'on appelle le sac embryonnaire, de forme variable, tantôt renflée, tantôt ayant l'aspect d'un tube grêle. Vers le sommet du sac embryonnaire se montrent, comme appendus à sa voûte, trois cellules, dont deux supérieures n'ont à jouer dans le développement de l'embryon qu'un rôle secondaire et sont appelées cellules synergiques; tandis que la troisième, située immédiatement au-dessous d'elles et dans l'intérieur de laquelle se développera un peu plus tard l'embryon, est la vésicule embryonnaire proprement dite ou l'œuf, si l'on veut se servir d'une expression empruntée au règne animal.

D'autre part, à la partie inférieure du sac embryonnaire se voient trois cellules, appelées cellules antipodes.

Strue. - Cette partie du gynécée (fig. 111, g) manque Forme. parfois, et alors le stigmate, qui n'est autre que sa partie terminale, est sessile, c'est-à-dire qu'il repose directement sur l'ovaire comme c'est le cas du Pavot (fig. 95, I, $n$ ).

Sa forme est ordinairement cylindrique, grêle, quelquefois étalée en lame, pétaloïde, comme dans l'Iris.

Il y a autant de styles que de carpelles, de sorte que, malSoudure. gré la soudure de ceux-ci, on peut savoir combien il en entre

Changements qui se passent dans l'ovule

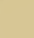


dans la constitution d'un ovaire. Mais les styles ne sont pas toujours libres; souvent ils sont soudés en totalité ou sur une longueur variable, et alors, suivant le nombre de leurs divisions, on les dit bifides, tmfides, etc.

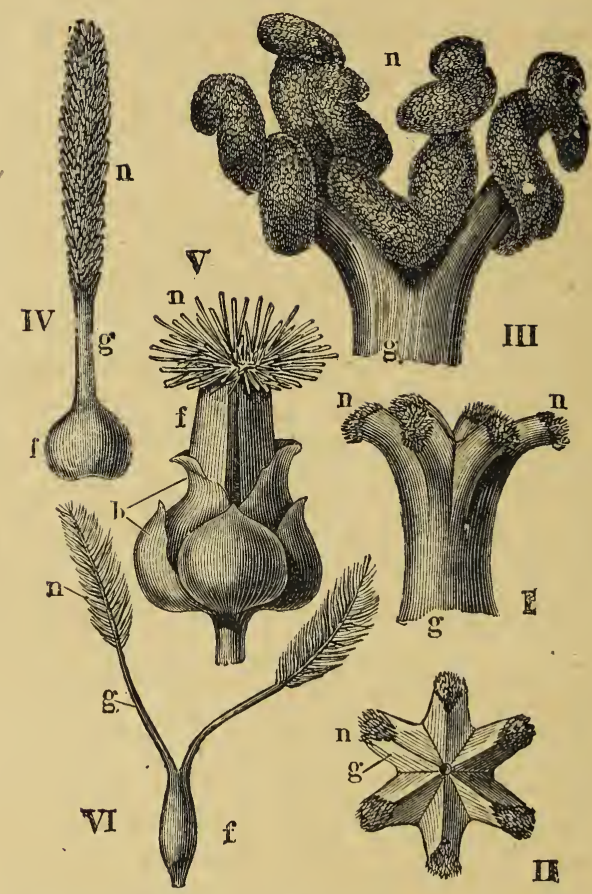

Fıg. 111. - Différentes formes de styles et de stigmatès; I, II, Cabaret (A sarum curopoum) vue de profil et de face (gr.4); III, Begonia (gr.6); IV, Plantain (Plantago major) (gr. 6); V, Troscart (Triglochin maritimum) (gr. 10); VI, Roseau (Arundo donax) (gr. 4); $-b$, périanthe; $f$, ovaire; $g$, style ; $n$, stigmates.

Situation.

Ordinairement terminal, c'est-à-dire, inséré au sommet des carpelles, qu'il continue, le style est parfois fixé à un niveau moins élevé, ce qui lui vaut le nom de latéral (Pommier, fig. 108, D); d'autres fois même, attaché à la base des carpelles, il est dit basilaire, disposition qui conduit au style gynobasique des Borraginées et Labiées, lequel apparaît en- 
foncé dans une dépression centrale creusée entre les carpelles qui seelèvent autour de lui, de sorte qu'on le dirait non plus inséré sur l'ovaire, mais directement porté par le réceptacle.

Après la fécondation, il se flétril le plus souvent, tombe, Évolution. et est dit caduc; d'autres fois il est persistant (Crucifères); parfois, enfin, il augmente de dimension, est accrescent, comme dans les Anémones, où il se développe sous forme d'une queue plumeuse, qui reste fixée au fruit et qui joue le rôle d'organe de dissémination.

Structure du Strle. - Cette partie du carpelle est composée de cellules allongées, lâchement unies entre elles et désignées sous le nom de tissu conducteur, parce que c'est entre elles, comme nous le verrons bientôt, que cheminent les tubes polliniques au moment de la fécondation, depuis le stigmate jusqu'à la cavité de l'ovaire. On y trouve aussi, comme il a été dit, des faisceaux fibrovasculaires; le tout est recouvert par l'épiderme.

Stignate. - Le stigmate (1), organe glanduleux qui surmonte le style, est sessile quand son support fait défaut; il repose alors directement sur le carpelle (fig. 9ð, $I, n$ et 111 , $V, n)$. Sa surface est comme veloutée, papilleuse et imprégnée d'une sécrétion visqueuse.

Il est simple ou multiple, selon le nombre des carpelles; quand il y en a plusieurs, les stigmates peuvent être tout à fail distincts ou bien soudés dans une étendue variable, mais toujours on peut y reconnaître des divisions qui indiquent le nombre des carpelles. Suivant le nombre et la profondeur de ces divisions, on dit les stigmates bilobés, trilobés, etc., lifides, trifides, etc.

Structure du stignate. - Le stigmate est formé de cellules prolongées en manière de papilles (fig. $111, n$ ), qui lui

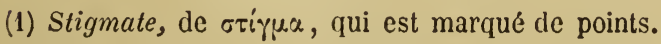


donnent son aspect velouté ou mamelonné ; au moment de la fécondation, les grains de pollen tombent sur ces papilles, où ils sont retenus par une humeur visqueuse sécrétée par ces cellules et propre à les faire germer, c'est-à-dire à provoquer la formation du tube pollinique. 


\section{CHAPITRE IX}

\section{Quelques notions de Morphologie végétale (1).}

10 Origine des parties de la Fleur; ce qu'on entend par Métamorphoses ascendante et descendante. $-2^{\circ}$ La symétrie dans la Fleur. La loi d'alternance. Diagramme et Plan de symétrie de la Fleur.

I. Origine des parties de la Fleur.

QUelqueS MOTS SUR L'HISTORIRUE DE LA QUESTION. - Le grand botaniste suédois Linné avait été frappé de ce fait que les êtres de la création offrent de l'un à l'autre, quand on les considère dans leur ordre naturel, de telles ressemblances organiques, qu'il est souvent très difficile, parfois impossible, de tracer entre eux des lignes de démarcation absolue pour les répartir en différents groupes, et de délimiter d'une façon certaine les espèces, les genres, les familles, etc.; "Natura non facit saltus », la Nature établit entre les différents êtres des transitions bien ménagées, disait-il avec grande raison.

Bien plus, cette ingénieuse remarque s'applique avec autant de vérité aux parties d'un mêrne organisme. Il semblerait que la nature répugne à produire de toutes pièces des parties nouvelles, mais qu'elle se contente souvent de modifier celles qui existent déjà pour les rendre aptes à remplir des fonctions différentes.

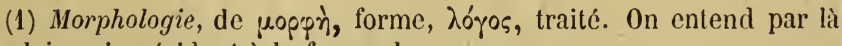
les lois qui président à la forme des organes. 
Il ne serait pas difficile de trouver dans le règne animal des preuves multiples qui justifieraient cette manière de voir. Le règne végétal va suffire à sa démonstration.

Le grand poète de l'Allemagne, Gœethe, qui était en même temps un savant naturaliste, eut le mérite de grouper en quelque sorte en un corps de doctrine les observations recueillies avant lui sur ce sujet et de fonder (1790) ce qu'il appella la théorie de la métamorphose, qu'il divise en ascendante ou progressive, et descendante ou régressive. Avant lui, un botaniste de Hambourg, Jungius (1678) avait déjà indiqué le sujet; un botaniste français, Adanson, avait émis l'avis que les sépales ne sorıt que des feuilles modifiées;; Fréd. Wolff (1759) avait admis que tous les organes que porte la tige, ${ }^{\circ}$ si variés qu'ils soient, sont en somme de même nature.

On peut, en effet, lpar des exemples bien choisis et pris sur diverses plantes, montrer que de la feuille dérivent par des transitions insensibles tous les autres organes appendiculaires, si bien que théoriquement l'on est autorisé à les considérer tous comme des feuilles modifiées, ou, si l'on veut, que les feuilles et les autres organes portés par la tige sont morphologiquement identiques.

Transformation des feuilles en sépales.
Exemples de Métamorphose ascendante. - Si l'on examine un pied de Pivoine, on voit que les feuilles en sont profondément découpées et décomposées, surtout celles de la base de la tige, et que les fleurs ont un calice dont les folioles ou sépales n'ont, au premier abord, aucun rapport avec les premières. Et cependant, à mesure que l'on envisage des feuilles placées plus haut sur la tige et se rapprochant davantage de la fleur, on les voit devenir, par degrés, plus simples et plus petites, de façon à ne plus présenter qu'un petit nombre de folioles, puis trois, puis une seule, de sorte que celles qui arrivent dans le voisinage dı périanthe sont réduites à un limbe unique, qui commence à s'élargir par sa base et tend à prendre une forme sépaloïde, tandis que sa partie supérieure se rétrécit; celles qui sont encore plus rapprochées de la fleur accusent 
davantage la forme de sépales et ne se distinguent plus de l'enveloppe extérieure de la fleur.

L'examen de la Pivoine nous fait donc assister aux modifications successives éprouvées par les feuilles pour devenir des sépales, ou si l'on veut, à la transformation progressive des feuilles en sépales.

Voyons maintenant un 'exemple du passage des sépales aux pétales. Considérons pour cela une fleur de Magnolia. Transformation
des sépales en pétales.

Nous voyons que le périanthe se compose de larges folioles qui ne se distinguent par aucun caractère essenticl les unes des autres; et cependant les plus extérieures constituent le calice, les intérieures, la corolle, de sorte que la délimitation est impossible à fixer entre ces deux sorles d'organes, qui, chez un très grand nombre de plantes, different cependant par des caractères manifestes.

On pourrait citer bien d'autres exemples démonstratifs empruntés aux Calycanthus, Hortonia, Badianes, et surtout aux Camélias, principalement ceux qui ont des fleurs doubles.

Les pétales et les étamines fournissent de nombreuses Transformation observations du même gẹnre, de sorte que ces deux espèces

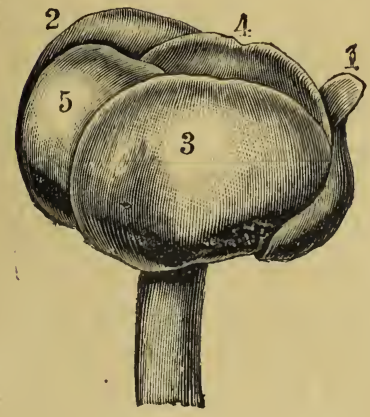

$\mathbf{I}$

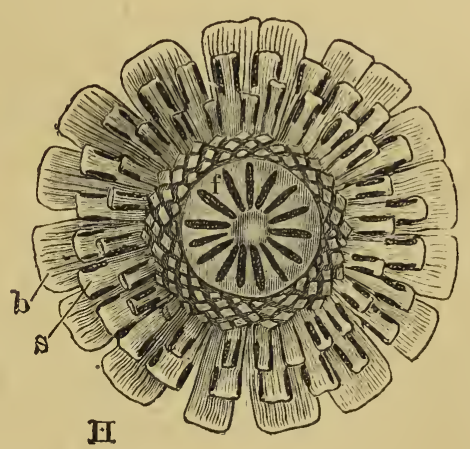

II

Fig. 112. - Fleur de Nénuphar jaune (grand. nat.); I, vue de profil, 1, 2, 3, 4, 5, les sépales; II, vue de face; $b$, pétales; $s$, organes moitié pétales et moitić étamines; $f$, ovaire.

d'organes floraux, qui semblent si essentiellement différents 
l'un de l'autre, peuvent être, en somme, considérés comme étant morphologiquement de même nature et dérivés des feuilles, l'un et l'autre.

La belle fleur du Nénuphar blanc (Nymphcea alba), ou celle du Nénuphar jaune (Nymphcea lutea, fig.112 et113), peut servir'à cette démonstration. Si l'on examine les pétales les plus intérieurs, on ne peut manquer d'observer qu'ils sont plus petits que les extérieurs, ct que l'amoindrissement s'opère par degrés de ceux-ci à ceux-là; puis, on remarque sur le sommel de certains de ces derniers une petite saillie qui devient plus développée sur les suivants, où elle offre franchement l'aspect d'une anthère; sur des pétales encore plus intérieurs, l'anthère augmente de volume et atteint enfin tout son développement normal, en même temps que son support, d'abord large et pétaloïde, se rétrécit et se réduit finalement à un étroit filet. Il résulte donc de ces faits, qu'à un moment donné, l'organe observé est moitié pétale et moitié étamine.
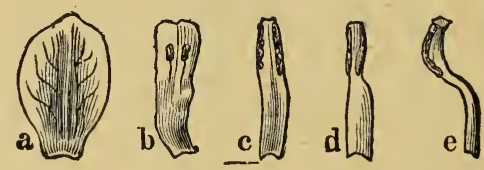

Fig. 113. - Transformation des pétales en étamines, dans le Nenuphar jaune; de $a$ à $e$ on en suit tous les passages.

Le pistil dérive également de la feuille.

Ce qui a étć dit des organes floraux étudiés jusqu'ici trouve encore son application en ce qui regarde le pistil. Comme les sépales, les pétales et les étamines, les carpelles peuvent être considérés comme étant des modifications des feuilles. Leur structure, comme nous l'avons vu dans un précédent chapitre, a une frappante analogic avec celle des organes foliaires, et si l'on suit la façon dont ils se montrent et se développent, on les voit apparaître sous l'aspect d'un mamelon, qui s'étale en forme d'une petite feuille dont les bords se rapprochent ensuite et se soudent pour former 
une cavité, en même temps que leur extrémité supérieure s'allonge pour constituer le style. D'ailleurs, les carpelles de certaines fleurs, des Sterculia, des Lunaires, par exemple, offrent d'une façon frappante l'apparence d'une véritable feuille.

Bien plus, il n'est pas rare dans les fleurs de certaines plantes de voir les étamines se transformer en carpelles; celles, par exemple, du Pavot, de la Joubarbe des toits, du Rosier; les plus intérieures sont devenues des carpelles en tout semblables aux carpelles normaux, ou bien ont un caractère mixte et portent à la fois du pollen et des ovules.

Exemples de Métamorphose descendante. - Il ne serait pas plus difficile de citer d'assez nombreux faits de métamorphose descendante ou régressive. Qui n'a vu de ces abominables roses verles, recherchées comme un objet de curiosité, et dans lesquelles les pétales et les étamines, sous l'influence de certains procédés de culture, se sont tous transformés en de petites feuilles de couleur verte?

Les étamines prennent souvent la forme de pétales; c'est en déterminant ce résultat que l'on arrive à obtenir nombre de fleurs doubles. La Rose sauvage (Rosa canina) n'a
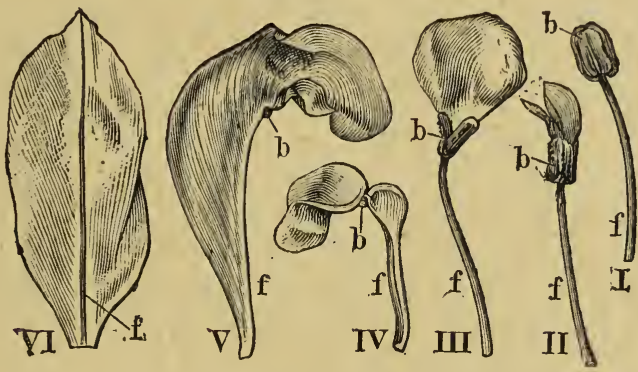

Fig. 114. - Transformation d'étamines en pétales (Scringat). On suit toutes les transitions entre ces deux sortes d'organes en examinant les dessins de I à VI; $f$, filet staminal ; $b$, anthère.

que cinq pétales et un très grand nombre d'étamines; par la culture les étamines deviennent des pétales, et il est 
très commun de voir de ces organes seulement à demi transformés. Les Anémones doubles, les Cerisiers doubles offrent constamment cette métamorphose de leurs étamines en pétales. Les carpelles, enfin, se transforment assez souvent en étamines, en pétales ou en feuilles vertes; le Trèfle en offre un bon exemple.

Remaroue. - On doit voir, d'après l'exposé de ces faits, ce qu'il faut entendre, en Botanique, par le mot,métamorphose. Il est bien clair qu'on aurait tort de le prendre dans le sens qu'on attache à cette même expression, quand il s'agit de désigner certains phénomènes qui se passent chez nombre d'animaux, où l'on voit un même être prendre successivement diverses formes. Rien de semblable, en réalité, n'a lieu dans la Plante. Nous n'y voyons pas, en effet, des organes déjà développés et de forme arrêtée qui tout à coup l'abandonnent, pour en revêtir une autre; mais nous trouvons des organes qui, au lieu de se montrer avec les caractères qu'ils offrent dans la fleur normale, présentent à un degré plus ou moins marqué la forme caractéristique qui appartient, dans la règle, à d'autres parties de cette même fleur.

Pour achever de faire comprendre la théorie de la Métamorphose, ajoutons que la fleur doit être en somme regardée comme une tige, ou sclon l'expression généralement adoptée, comme un axe pourvu d'appendices. Mais cet axe est très raccourci, tellement qu'il est obligé de s'élargir pour fournir insertion aux nombreux organes qu'il porte; c'est sur cet élargissement, qu'on appelle réceptacle, et dont la surface peut être plane, convexe ou concave (voy. fig. 108), que sont groupées et nécessairement très rapprochées les unes des autres toutes les parties de la fleur; ainsi au lieu d'être superposées comme le sont les feuilles, ces parties se trouvent disposées par zones concentriques ou suivant une spirale. En outre, ces appendices, au lieu d'avoir la forme ordinaire des feuilles, sont très diversifiés à ce point de vue, dans le but de remplir des rôles différents, de sorte qu'au premier abord la fleur paraît constituée par un 
ensemble d'organes d'une nature toute spéciale et n'ayant aucun rapport avec l'organisation des appendices foliaires portés par la tige proprement dite.

II. La Symétrie dans la Fleur

Principale loi : Alternance entre les parties de deux verticilles voisins. - De même que les feuilles, bien loin d'être placées au hasard sur la tige, sont disposées d'une façon fort régulière, les organes floraux, qui en dérivent, occupent aussi sur l'axe raccourci qui les porte des situations toujours subordonnées à certaines règles.

Si l'on prend comme type, pour fixer les idées, une fleur qui, comme celle de Grassule (fig.11วั), soit aussi régulière que

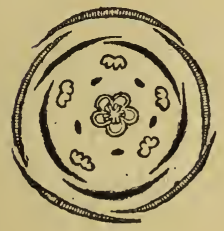

Fig. 115.

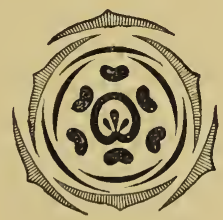

Fig. 116.

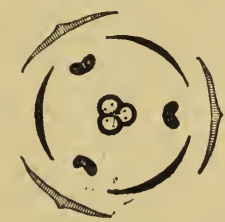

Fig. 117.

Fig. 115, - Diagramme d'une fleur de Crassule.

Fig. 116. - Diagramme d'une fleur de Berbéris.

Fig. 117. - Diagramme d'une fleur d'Iris.

possible el composée de quatre verticilles successifs, qui sont, en allant de dehors en dedans, le calice, la corolle, l'androcée ou l'ensemble des étamines et le pistil ou l'ensemble des carpelles, on voit que les parties de l'un des verticilles, quel qu'il soit, alterne avec les pièces du verticille suivant, c'est-à-dire, que l'insertion d'une pièce quelconque d'un verticille répond à l'intervalle de deux pièces du verticille adjacent. Ainsi, la fleur de Crassule offre un calice de cinq sépales, une corolle de cinq pétales alternant avec les précédents, un androcée de cinq éta- 
mines alternes avec les pétales, et un pistil de cinq carpelles alternant de même avec les étamines.

L'alternance des verticilles successifs, telle est donc la principale loi de la Symétrie florale.

lexceptions à la loi daalternance.

Mais cette loi offre de si nombreuses exceptions, elle est si souvent altérée, qu'il y a en réalité fort peu de fleurs où elle trouve son application entière et absolue. Chez un grand nombre on en observera des exemples partiels: ainsi, dans la plupart des cas, les pétales alternent avec les sépales, tandis qu'en même temps les deux autres verticilles sont opposés ou, comme on dit encore, superposés, entre eux ou par rapport aux pétales, c'est-à-dire, que l'insertion de deux pièces voisines appartenant à des verticilles différents se trouve sur une même ligne qui irait du centre de la fleur à la périphérie.

Il faut remarquer que parfois l'alternance existe, mais se trouve en quelque sorte masquée par des circonstances particulières : c'est ainsi que dans la fleur du Berberis les pétales semblent superposés aux sépales; mais un examen moins superficiel montre que le calice se compose en réalité de deux verticilles aussi bien que la corolle, et que le verticille intérieur de la corolle alterne avec celui qui est extérieur, celui-ci avec le verticille intérieur du calice, lequel alterne lui-même avec celui qui est le plus en dehors (fig. 116).

L'alternance n'est pas plus absolue pour les carpelles ; c'est ainsi que dans le Lis, la Jacinthe, l'Iris et d'ailleur's dans la plupart des Monocotylédones, les carpelles ou, si l'on veut, les loges de l'ovaire, sont opposés aux sépales extérieurs, tandis que, dans les Dicotylédones, ils sont tantôt alternes et tantôt opposés.

Il arrive même que des plantes appartenant à une même famille naturelle offrent une semblable divergence au point de vue de la situation relative des carpelles et des sépales.

Circonstances qui masquent la symétric florale.
Il ne faut pas oublier, par ailleurs, que la disposition des parties se modifie souvent avec le développement complet de la fleur, de sorte que, si l'on veut être renseigné exactementsur les relations vraies des parties,il faut les considérer avant l'épanouissement de la fleur, c'est-à-dire, dans le 
bouton. C'est ainsi que dans la fleur de l'Ancolie, où l'on voit un grand nombre d'étamines disposées sur dix rangées

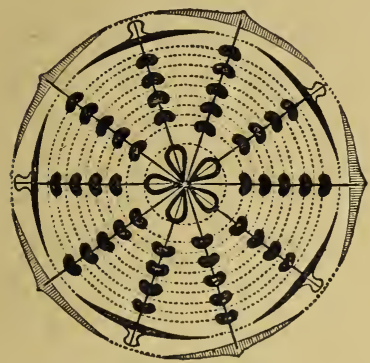

Fig. 118. - Diagramme d'unc fleur d'Ancolie.

rayonnantes, dont cinq sont opposées aux pétales et cinq alternes avec eux, l'alternance existe en réalité pour les unes comme pour les autres, car si on les étudie dans le bouton, on voit que les étamines de cette fleur sont disposées sur un grand nombre de verticilles, dont le plus extérieur alterne avec les pétales et tous les autres alternent entre eux.

Enfin, faisons remarquer, en terminant, quelques autres circonstances qui font varier d'une façon absolue la symétrie florale ou tout au moins peuvent la masquer à l'œil de l'observateur : telles sont l'augmentation du nombre des pièces d'un verticille par multiplication ou dédoublement; le Pavot (fig. 95), par exemple, qui n'offre que deux pièces au calice, en a quatre à la corolle, de sorte que la fleur semble asymétrique; mais il n'en est rien, la corolle étant en réalité formée de deux verticilles, alternant l'un avec l'autre, et l'extérieur avec le calice.

La soudure des pièces d'un même verticille entre elles, l'avortement ou l'atrophie d'une ou plusieurs pièces, ou même la disparition d'un verticille tout entier, sont encore autant de causes qui viennent souvent modifier la symétrie de la fleur.

Diagrame et Plan de symétrie de la fleur. - Les botanistes, pour figurer la position relative des parties d'une fleur, ont adopté, comme on l'a vu déjà, un dessin fort simple, qui se prête à toutes les modifications possibles. C'est ce qu'on appelle un diagramme (1), lequel n'est

(1) $\Delta\llcorner\alpha ́ \gamma p \alpha \mu \mu \alpha$, dessin, figure géométrique. 
autre chose qu'une projection, sur un plan, des verticilles de la fleur, dont chaque partie est représentée par un trait de convention.

Le diagramme d'une fleur de Grassule (fig. 115), déjà citée comme très régulière, offre un premier cercle de lignes en forme de croissant, qui figurent le calice, puis un second concentrique au premier, d'une autre teinte, représentant la corolle; en dedans de celui-ci, le cercle des étamines, dont les anthères biloculaires sont indiquées; entre deux étamines voisines se trouvent de petits organes grandulaires (disques) qui sont également figurés par un petit ovale; enfin au centre du diagramme se voient les loges de l'ovaire, dans l'angle de chacune desquelles se remarquent deux ovules.

Un tel diagramme permet non seulement de reconnaître le mode de préfloraison, c'est-à-dire, la façon dont les organes se recouvrent dans la fleur avant l'épanouissement, le mode d'insertion des différentes parties relativement les unes aux autres, a utrement dit, leur situation alterne ou opposée, la direction introrse ou extror'se des étamines, à savoir, si le côté où se fait la déhiscence regarde en dedans ou en dehors de la fleur, etc.; mais il fait de plus reconnaître, au premier coup d'ceil, la symétrie de la fleur,

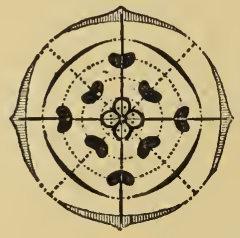

Fig. 119.

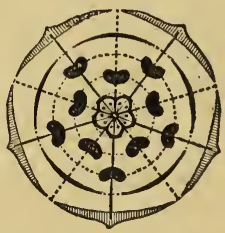

Fig. 119. - Diagrammes? de fleurs dicotylédones sur le type quatre et sur le type:cinq.

Fig. 120. - Diagramme de fleur monocotylédone sur le type trois.

c'est-à-dire, dans quelle direction il faut faire passer un plan vertical, pour que la fleur soit divisée en deux parties égales. Dans une fleur, comme celle qui nous sert de type, on peut voir qu'il existe un grand nombre de plans symé- 
triques, car toute section qui passera par le centre de la fleur et par le milieu d'un sépale ou dans l'intervalle de deux sépales divisera cette fleur en deux moitiés parfaitement comparables entre elles; il y aurait donc dans ce cas dix plans de symétrie. Cette fleur est pentamère, chaque verticille se composant de cinq parties; dans une fleur régulière trimère ou à trois divisions par verticille, on aurait de même six plans de sy-

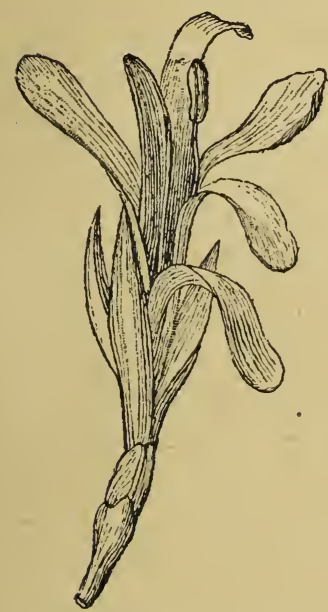

Fig. 121. - Fleur asymétrique de Balisier. métrie, et huit dans une fleur tétramère.

Il n'en est plus de même dans un grand nombre de fleurs qui sont plus ou moins irrégulières, les pièces des verticilles n'étant pas en nombre égal ou en progression multiple. Le seul plan de symétrie, qui existe alors, est le plus souvent celui qui passe à la fois par le centre de la fleur, par le milieu de la tige qui la porte, et par le milieu de la feuille ou bractée à l'aisselle de laquelle cette fleur a pris naissance.

Enfin, il y a des fleurs qui n'offrent aucun plan de symétrie, c'est-à-dire, dont la structure est tellement irrégulière, qu'il est impossible de les diviser en deux parties égales, dans quelque sens que l'on en pratique la coupe : telle est celle du Balisier. 


\section{CHAPITRE X}

\section{Fécondation des Végétaux.}

Pollinisation; procédés divers au moyen desquels cette opération s'accomplit. Germination du pollen et Fécondation proprement dite. Évolution de l'ovule après sa fécondation. - Fécondation et développement de l'Embryon chez les Gymnospermes. - Hybrides et Métis.

Pollinisation; Procédés mis en oeuvre pour son accomplissement. - Au moment où se produit la déhiscence des anthères, les grains de pollen s'échappent au dehors en nombre immense. Si beaucoup sont perdus, dispersés par le vent ou la pluie, un certain nombre d'entre eux tombent sur le stigmate, où ils sont retenus par la sécrétion visqueuse de cet organe, d'où ils iront bientôt féconder les ovules.

La pollinisation, c'est-à-dire le contact du pollen avec le stigmate, étant une opération très importante, voyons par quels moyens son succès est assuré dans la nature.

Pollinisation dans les fleurs hermaphrodites.

Dans un grand nombre de plantes les étamines el le pistil sont réunis sur la même fleur (fleurs hermaphrodites), de sorte que le 'pollen n'a pas grand chemin à faire pour aller trouver le stigmate et que, dans beaucoup de cas, l'autofécondation, c'est-à-dire la fécondation de la fleur par elle-même, ne présente pas de difficulté. Bien plus, dans certaines de ces fleurs, celles de la Rue, du Berbéris, on voit les étamines s'incliner d'elles-mêmes ou sous l'influence de la moindre irritation vers le pistil pour y déposer leur pollen; d'autres fois, le pistil en s'allongeant frotte nécessairement contre les anthères disposées 
en un tube étroit qui l'embrasse, comme dans le Haricot, et retient aveclui une certaine quantité de pollen.

Mais il faut se hâter de reconnaître que de semblables dispositions sont loin d'être le résultat d'une règle absolue, à ce point que, même dans ces fleurs hermaphrodites, l'autofécondation est souvent très difficile.

Ainsi, il arrive assez souvent que les étamines et le pistil ne se trouvent pas, au même moment, dans les conditions favorables à la fécondation, les unes arrivant à leur maturité avant l'autre ou inversement, particularité qui a été désignée sous le nom de dichogamie. En pareil cas, la pollinisation ne peut être qu'indirecte, c'cst-à-dire, ne peut se produire qu'entre deux fleurs voisines, dont le pistil de l'une et les étamines de l'autre se trouvent dans un état de maturité convenable.

D'autres fois, dans une fleur dressée, par exemple, le pistil, très long, dépasse beaucoup les étamines, de sorte que leur pollen ne saurait l'atteindre; ou bien, dans une fleur renversée, la disposition contraire a lieu, les étamines dépassant notablement le stigmate. Dans tous ces cas et dans bien d'autres la pollinisation directe est très difficile.

Un très grand nombre de plantes sont monoïques, c'està-dire que, sur une même plante, certaines fleurs ne portent Pollinisation dans les fleurs que des étamines et d'autres que des pistils. Dans ces cas, on le comprend, la pollinisation ne peut être qu'indirecte.

A plus forte raison en est-il de même pour les espèces dioïques, c'est-à-dire, celles dont les fleurs mâles et les fleurs femelles sont portées par des individus différents.

Dans de semblables circonstances la pollinisation ne peut être accomplie que par l'intermédiaire d'agents étrangers. Le vent joue un rôle considérable dans la dissémination de la poussière fécondante, qu'il transporte même à de grandes distances. Pour n'en citer qu'un exemple, il suffira de mentionner l'Aucuba du Japon, arbuste ornemental à feuillage d'un beau vert, répandu dans nos jardins depuis un petit nombre d'années. Cette espèce est dioïque et les individus mâles sont beaucoup moins répandus que les individus femelles, ce qui n'empêche pas ceux-ci d'être

Agents étranger's à la fleur qui favorisent la pollinisation. 
fécondés et de mûrir leurs jolis fruits rouges comme des cerises. Parfois, le vent entraîne à des distances plus ou

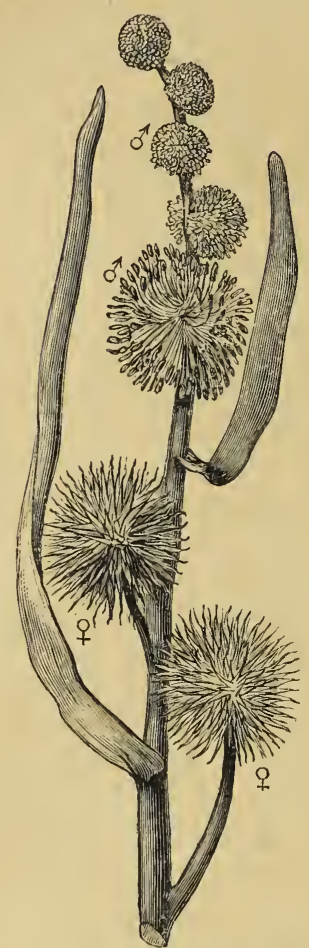

Fig. 122. - Inflorescence de Sparganium (fam. des $\Lambda$ roödées) dont les fleurs mâles ( $\left.\sigma^{1}\right)$ occupent la partic supéricure, les lleurs femelles ( $Q$ ) la partic inféricure. moins considérables des masses de pollen enlevées aux arbres des forêts et les répand dans les campagnes, où on les désigne vulgairement sous le nom de pluies de soufre.

Certaines dispositions particulières favorisent la pollinisation dans les plantes monoïques ou dïoïques. Ainsi dans le Maïs, les Carex et d'autres, lesfleursfemelles sont placées au-dessous des fleurs mâles sur un même rameau, et le pollen, en tombant des premières sur les secondes, vient les féconder.

Ia Vallisnérie spirale, plante aquatique, mérite entre toutes une mention spéciale; elle est dioïque, et ses fleurs sont toutes submergées. A un moment donné, les fleurs mâles rompent leurs pédoncules, et, devenues libres, remontent à la surface de l'eau; d'autre part, les pédoncules des fleurs femelles s'allongent considérablement pour permettre ì celles-ci d'arriver à la surface, au milieu des précédentes, qui les fécondent alors. Lorsque la pollinisation est accomplie, les pédoncules des fleurs femelles se contractent en forme de spirale, ce qui oblige celles-ci à revenir au fond de l'eau, où elles mûrissent leurs fruits.

Rôle Mais les agents les plus actifs peut-être de la pollinisades Insectes. tion, ce sont les Insectes, qui, en passant vingt fois dans une minute d'une fleur à l'autre, pour sucer le liquide 
sucré produit dans les nectaires (fig. 123), transportent avec eux le pollen qui s'altache à leur corps. Leur rôle paraîl
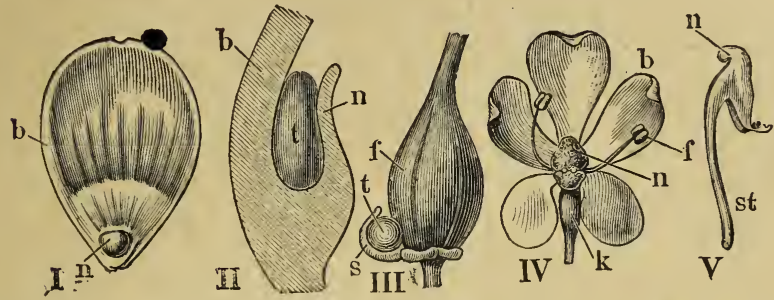

Fig. 123. - Nectaires de diverses fleurs; I, de Renoncule (Ranunculns acer) (gros. 3) ; II, le même, en coupe tongitudinale (gros. 8); lII, de Rhinanthe (Rhinanthus major) (gros. 3); IV, d'Anthrisque (Anthriscus sylvestris) (gros. 6) ; V, d'Aconit (Aconitum napellus) (grand. nat.); - $n$, nectaire; $b$, pétale; $t$, liquide sucré; $f$ (de la fig. III) et $k$, ovaire; $f$ (de la fig. IV), étamine; $s l$, pédoncule.

incontestable dans la fécondation des plantes dioïques, monoïques, et dans beaucoup d'espèces même hermaphrodites, comme celles qui présentent la dichogamie, dont il a été question plus haut, ou bien une disposition telle que le pollen ne peut se répandre facilement sur le pistil, comme la Sauge des prés (fig. 124) en offre un exemple remarquable.

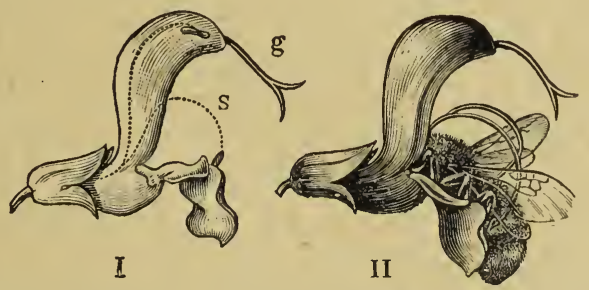

Fig. 124. - Fécondation de la Sauge des prés par l'intervention d'un Bourdon; I, fleur de Sauge, dont on voit le style très proéminent $(g)$, tandis que les étamines $(s)$ sont bien plus courtes et cachées daus la fleur; II, le Bourdon s'introduit dans la fleur pour en sucer le nectar, et fait basculer les étamines, dont les anthères viennent frotter sur le dos de l'insecte et y déposer le pollen qui, lorsque celui-là pénétrera dans une autre fleur semblable, sera facilement mis au contact du stigmate.

La fécondation artificielle des Figues, telle qu'on la pratique en Kabylie, où ces fruits constituent une partie importante de l'alimentâtion, est un exemple de fécondation par l'intermédiaire des insectes, bien que, pendant longtemps, on 
n'ait pas su expliquer ce qui se passait dans cette méthode. Les fleurs du Figuier sont, comme on le sait, réunies en grand nombre sur un réceptacle commun, concave, à l'orifice duquel se trouvent les fleurs mâles, tandis que la cavité est occupée par les fleurs femelles. Or, les habitants du pays s'imaginent qu'en suspendant aux branches de leurs Figuiers des figues sauvages, dont la maturité est beaucoup plus précoce, ils obtiennent des fruits plus gros et plus succulents. Cette croyance n'est pas sans fondement, et voici à quoi est dê un semblable résultat. Les figues sauvages servent de retraite à une quantité de petits Hyménoptères du genre Cynips, qui pénètrent bientôt dans les inflorescences du Figuier cultivé, et par leurs mouvements assurent mieux le contact du pollen avec les stigmates des fleurs femelles; d'où il suit que la fécondation a lieu pour un plus grand nombre des fleurs de cette inflorescence, ce qui amène un développement plus considérable du fruit.

Dans les pays chauds les Oiseaux-mouches, les Colibris au long bec, jouent un rôle analogue à celui des Insectes dans nos climats.

Parfois, enfin, la pollinisation est assurée par les soins mêmes de l'homme. C'est ainsi que depuis un temps immémorial, puisqu'il faut remonter au delà d'Hérodote, les habitants du Sahara, pour assurer la complète récolte des dattiers, coupent les fleurs mâles de ces arbres et en introduisent la poussière fécondante dans la spathe qui enveloppe les fleurs femelles, portées par d'autres individus.

Une opération analogue se pratique à la Réunion, pour la fécondation des fleurs de la Vanille.

Enfin, lesjardiniers pratiquèrent assez souvent la fécondation artificielle, pour certaines plantes, les Orchidées, par exemple, qui ne seraient que difficilement fécondées par elles-mêmes, ou bien quand ils veulent maintenir certaines races dans toute leur intégrité primitive, ou au contraire obtenir des variétés, des hybrides, etc.

Germination du pollen et Fécondation proprement dite. - Lorsque les grains de pollen se trouvent mis en rapport 
avec le stigmate, ils ne restent pas inactifs. En effet, tandis que le contact de l'eau pure rompt les membranes du pollen

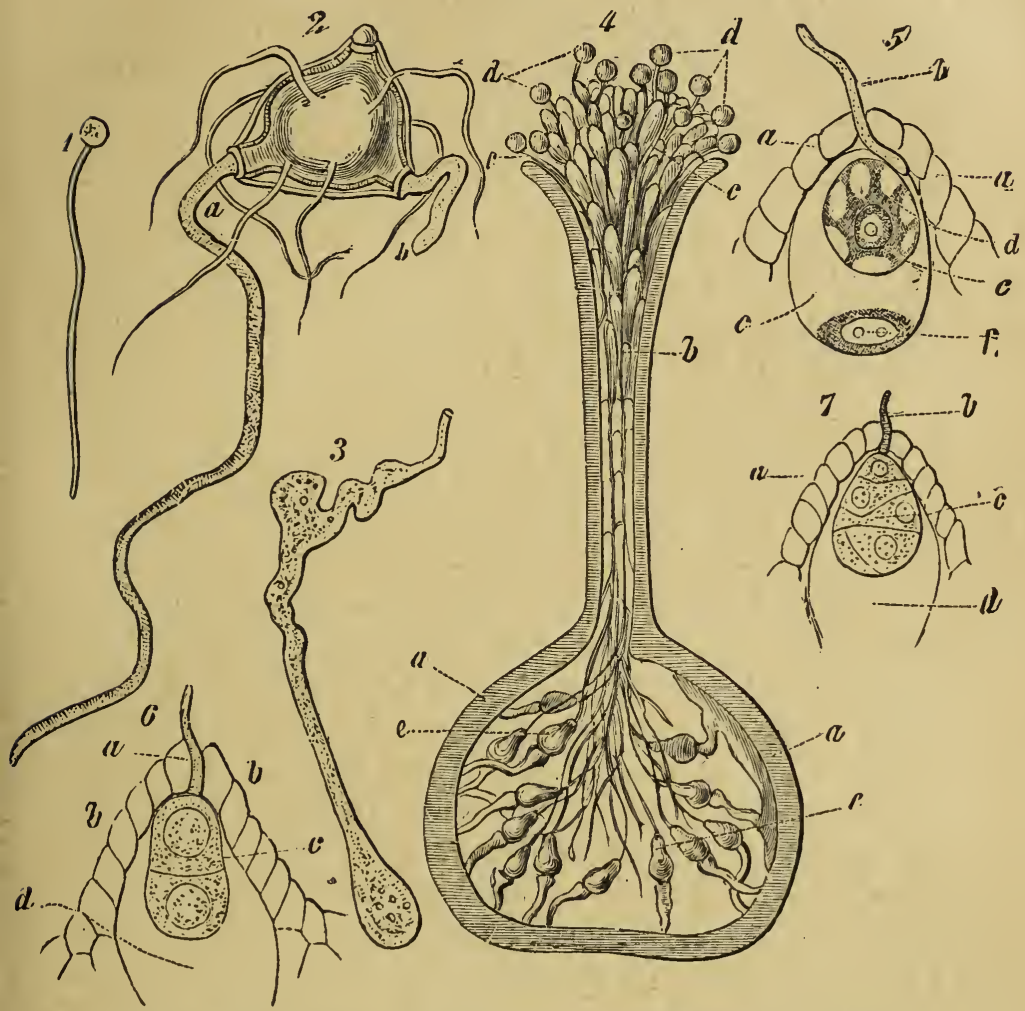

Fig. 125. - Germination du pollen et marche des phénomènes de la fécondation; 1, grain de pollen en voie de germination; 2, grain de pollen triédrique de l'Epilobium hirsutum, hérissé de longs filaments et pourvu de pores qui donnent passage à deux tubes polliniques $(a, b) ; 3$, un tube pollinique de Momordica elaterium, présentant des renflements irréguliers; 4, coupe longitudinale d'un pistil d'Hélianthême; $a$, paroi de l'ovaire ; $b$, tissu conducteur ; $c$, papilles stigmatiques; $d$, grains de pollen en germination ; $e$, ovules auxquels arrivent les tubes polliniques ; 5 , fécondation de l'ovule dans la Fritillaire impériale; $a$, paroi du nucelle; $b$, un tube pollinique; $c$, le sac embryonnaire; $d$, $e$, la cellule embryonnaire, qui deviendra l'œuf végétal; les deux synergides, qui n'ont pas été figurées, devraient la surmonter; $f$, cellules antipodes ; 6 , cellule cmbryonnaire après la fécondation ; elle s'est déjä divisée en deux cellules $(c)$; $a$, tube pollinique; $b$, paroi du nucelle; $d$, albumen ou endosperme; 7, embryon de Pistia ovata, dont le développement est plus avancé.

et laisse échapper en pure perte la fovilla qu'elles renfer- 
maient (ce qui a lieu fatalement quand, au moment de la floraison, des pluies abondantes se produisent), les ovaires n'étant pas fécondés ne grossissent pas; c'est cet accident que les jardiniers désignent sous le nom de coulure. Au contraire, le contact du suc visqueux qui imprégne le stigmate détermine la germination des grains de pollen. Ceuxci absorbent par endosmose une certaine quantité de liquide, lequel a pour effet de distendre leurs parois ; bientôt, au niveau d'un ou plusieurs des pores qu'ils présentent et où la membrane externe ou exhyménine manque, se fait une sorte de petite hernie, une saillie de l'endhyménine distendue par la fovilla, qui s'allonge de plus en plus, pour former le boyau ou tube pollinique.

Cette germination sc fait parfois alors que le pollen est encore renfermé dans l'anthère, surtout quand le stigmate étant très voisin de celle-ci, il peut l'humecter de sa sécrétion.

Les tubes polliniques s'enfoncent dans les interstices des papilles stigmatiques et suivent toute la longueur du style formé par le tissu conducteur, en se nourrissant de la substance des cellules au contact desquelles ils se trouvent. Ils arrivent ainsi, en plus ou moins grand nombre, jusquà la cavité de l'ovaire, selon la quantité d'ovules à féconder, car chacun de ceux-ci doit recevoir un tube pollinique.

La marche des tubes polliniques à travers le style est plus ou moins rapide; ainsi, dans le Safran, dont le style a une longueur de 5 à 10 centimètres, ils ne mettent qu'un à trois jours, tandis que pour celui de l'Arum, qui n'a que 2 ou 3 millimètres, il leur en faut cinq. Il est même des plantes, les Orchidées, où ils emploient des semaines et des mois pour arriver à destination.

Phénomènes intimes de la fécondation.

Quant le tube pollinique rencontre le sommet de l'ovule, il s'insinue par le micropyle et arrive au nucelle, au contact duquel il s'aplatit. La fovilla traverse alors la paroi du tube où elle était enfermée, puis la mince mombrane de l'une des synergides ou vésicules synergiques (voy. p. 173), pour pénétrer dans celle-ci. $\Lambda$ son tour, cette dernière laisse passer une partic de son contenu dans la cellule embryon- 
naire sous-jacente. Bientôt il se forme dans cette dernière un noyau, qui peu auparavant, n'y existait pas. On est porté à croire que ce noyau n'est autre que celui que renfermait le tube pollinique lui-mème, lequel se serait diffusé pour pénétrer à travers la membrane de la cellule embryonnaire et se condenserait aussitôt après. Quoi qu'il en soit, ce noyau se réunit bientôt à celui de la vésicule embryonnaire elle-même, pour n'en former qu'un seul.

A partir de ce moment, la fécondation est accomplie, l'ovule s'est transformé en cuf végétal, pour nous servir L'ovule devenu l'œuf végétal. d'une expression empruntée au règne animal; et dès lors il est susceptible de se développer en un nouvel individu semblable à celui dont il procède. Il va aussitôt se passer en lui toute une série de changements, qui aboutiront d'abord à sa transformation en graine.

Peu après les phénomènes qui viennent d'être exposés, l'une des vésicules synergiques, celle qui n'a pas pris part à la fécondation el dont le rôle est nul, se détruit, de sorte qu'il n'en reste plus trace.

Quant aux vésicules antipodes (voy.p.173), leurs fonctions dans le phénomène de la fécondation sont encore complètement inconnues.

Dès que l'ovule est fécondé, le micropyle se resserre, s'oblitère et étrangle le tube pollinique qui se résorbe sans laisser de traces.

Le rôle de la fleur est désormais achevé; le périanthe perd ses brillantes couleurs, se flétrit et tombe le plus souvent; parfois cependant il persiste, le calice surtout, qui même à partir de ce moment, peut prendre un plus grand développement, être, comme on dit, accrescent, et faire partie intégrante du fruit. Les étamines et le style se dessèchent et tombent, du moins dans la règle générale, car assez souvent celui-ci persiste et devient un organe de dissémination des graines.

Dès lors la fleur a cessé d'exister et le fruit commence son évolution (1).

(1) Pour les phénomènes chimiques qui accompagnent la floraison et la fécondation, voyez le Chapitre de la Respiration.

Evolution des autres partics de la fleur. 
Embryon

et filet suspenseur.

Parties constitutives de l'embryon.

Évolution de l'ovule a Partir de sa FÉCONDATION. - L'Ovule fécondé, devenu par conséquent un nouvel organisme, rudimentaire, il est vrai, l'œuf végétal, en un mot, ne tarde pas à s'allonger et à se diviser en deux cellules, par production d'une cloison transversale (fig. 125, 6, c). Le plus souvent, la cellule inférieure contribuera seule à former l'embryon, l'autre se transformant en un petit filet, dont la partie supérieure est fixée à la voûte du sac embryonnaire, tandis que l'inférieure porte l'embryon; on l'appelle, pour cette raison, le suspenseur.

L'embryon proprement dit ne tarde pas, par suite de son accroissementet de divisions intérieures successives, à montrer deux petites saillies (Dicotylédones) ou une seule (Monocotylédones), première trace des cotylédons, en même temps que d'autres cloisons divisent sa partie centrale en

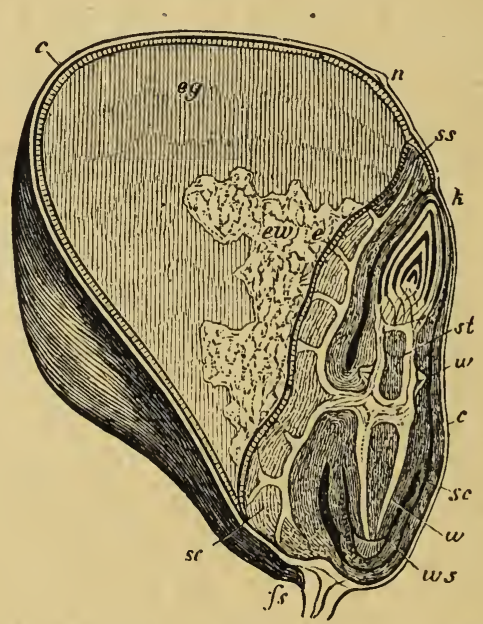

Fig. 126. - Coupe longitudinale du fruit très grossi du Maïs; $c$, péricarpe doublé du tégument de la graine; $n$, point où s'insérait le style $; s$, pédicule du fruit ; $e g, c w$, albumen, dur et janne à la périphérie, mou et blane vers le centre; $s s, s c$, cotylédon unique enveloppant l'embryon ; e, épiderme du cotylédon ; $s t$, tigelle ; $w$, radicule; $w s$, gaîne de la radicule; $k$, gemmule. Les lignes blanches qui parcourent l'embryon et le cotylédon représentent les faisceaux fibro-vasculaires.

plusicurs couches emboîtées les unes dans les autres représentant l'épiderme, l'écorce et le bois de la nouvelle 
plantule. Enfin, celle-ci s'allonge par ses deux extrémités et présente, d'un côté, la radicule et de l'autre, la tigelle, où l'on peut souvent même distinguer déjà un rudiment de bourgeon foliaire, la gemmule (fig. 126).

En outre, aussitôt la fécondation accomplie, le sac embryonnaire est le siège d'un travail, qui a pour effet d'y former un dépôt parfois très abondant d'une réserve nutritive qui servira au développement de l'embryon, et qu'on appelle albumen ou endosperme. Si l'embryon l'absorbe complètement pour subvenir à sa croissance, il n'en reste pas trace dans la graine mûre; mais souvent il en persiste une quantité plus ou moins considérable, dont l'aspect et la consistance lui ont valu les désignations de farineux ou amylacé (Graminées), oléagineux ou charnu (Ricin, Pavot), corné (Dattier, Caféier, Phytelephas); c'est même l'albumen de ce dernier qui constitue l'ivoire végétal, employé aux même usages industriels que l'ivoire animal.

Lorsque l'embryon devient très volumineux, il remplit tout le sac embryonnaire et digère tout l'albumen; mais les cotylédons qui se sont gorgés de matières nutritives aux dépens de celui-ci constituent à leur tour une réserve que l'embryon absorbera au fur et à mesure de ses besoins, au moment de la germination.

Indépendamment de cet albumen interne ou endosperme, formé dans le sac embryonnaire, il peuts'en former un second en dehors du premier, aux dépens du nucelle. Celui-ci, en effet, qui disparaît souvent en entier avant même la fécondation, persiste parfois et peut s'accroître en se gorgeant de substances nutritives; on donne à cet albumen le nom de périsperme. Il est tantôt de durée éphémère et promptement absorbé par l'embryon, comme dans l'Amandier, et tantòt plus durable, comme dans le Balisier. Enfin, l'endosperme et le périsperme peuvent exister simultanément; la graine est alors pourvue de deux albumens emboîtés l'un dans l'autre (Pipéracées, Nymphéacées).

Fécondation et développement de l'Embryon chez les Gymospermes. - L'histoire de la fécondation telle qu'elle 
vient d'être exposée, s'applique à la plupart des plantes phanérogames; mais il est un groupe spécial, qui offre des particularités importantes qu'on ne peut passer sous silence.

Ce groupe est celui des Conifères ou Arbres verts, encore désignés sous le nom de Gymnospermes (rup.vòs, nu, et $\sigma \pi \varepsilon ́ p \mu \alpha$, scmence), parce que leurs ovules ne sont pas renfermés dans des ovaires clos de toutes parts, contrairement aux plantes qu'on a eu en vue jusqu'ici, les Angiospermes,

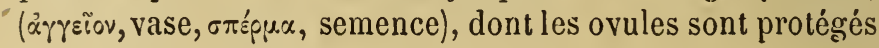

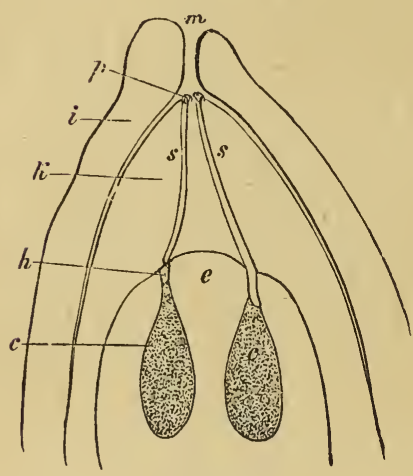

Fig. 127 .

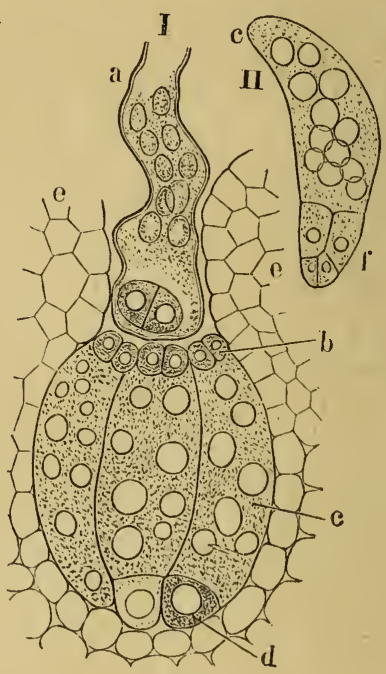

Fig. 128.

Fig. 12\%. - Vue schématique de la fécondation d'unc Conifère; $m$, micropyle $i$, enveloppe de l'ovule; $p$, grains de pollen envoyant chacun un tube pollinique $(s, s)$ aux corpuscules $(h, c) ; e$, albumen.

Fig. 128. - I, Corpuscules de Genévricr (Juniperus communis) (gross. 300 fois); $a$, tube pollinique; $b$, cellules en rosette des corpuscules; $c$, corpuscules; $d$, cellules embryonnaires; $e$, endosperme ; II, un corpuseule isolé $(c)$ avec l'cmbryon $(f)$ en voie de développement.

par une enveloppe complète. Aux Gymnospermes appartiennent les Pins, Sapins, Thuya, Cyprès, etc.

Leurs fleurs sont toujours unisexuées, leurs ovules toujours orthotropes et pourvus d'un tégument unique. Le 
nucelle offre un sac embryonnaire volumineux, dans lequel, longtemps avant la fécondation (ce qui établit une grande

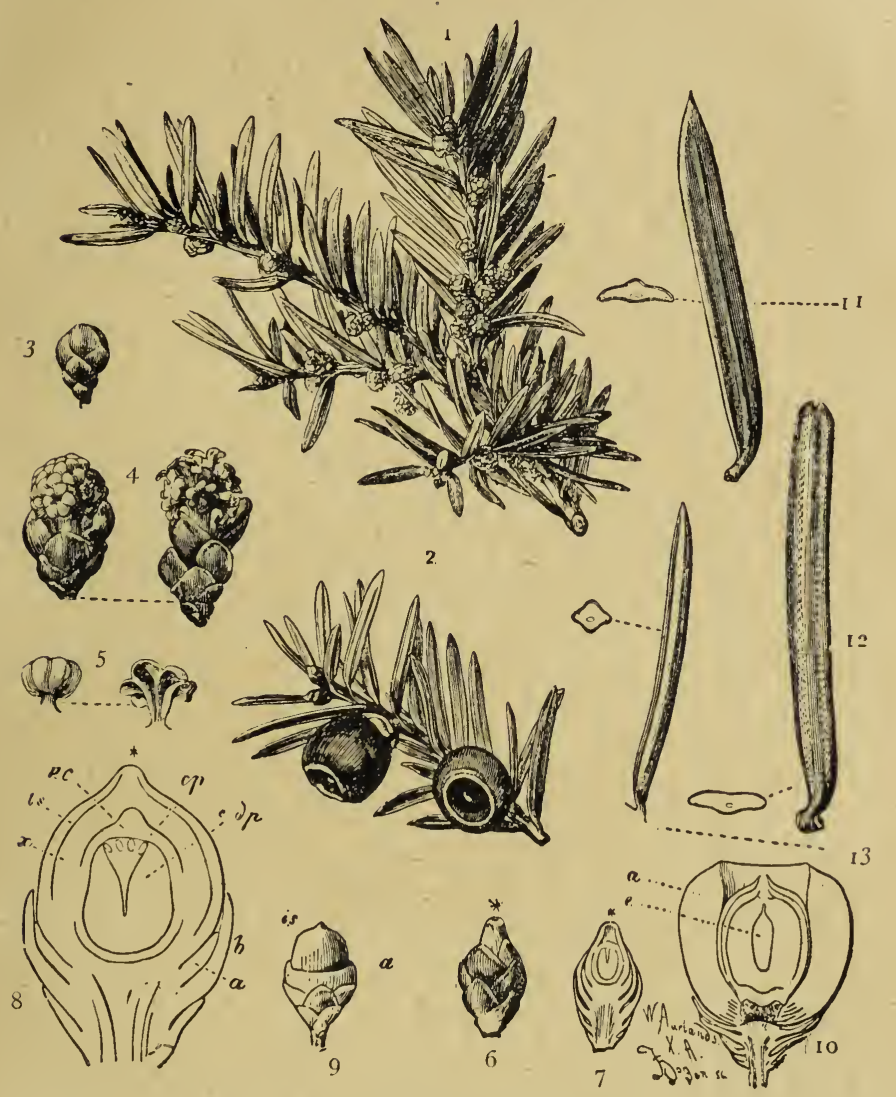

Fig. 129. - Organes de la reproduction d'une Conifère, l'If (Taxus baccata); 1, rameau chargé de fleurs mâles ; 2 , rameau portant deux fruits mûrs ; 3 , bouton de fleur mâle; 4, fleurs mâles épanouies montrant leurs étamines; 5 , étamines, l'une encore fermée, l'autre ouverte ; 6 , fleur femelle ; 7 et 8 , la même, fendue en long, vue sous deux grossissements différents; $l s, x$, enveloppe de l'ovule devenant en partie ligneuse; $n c$, sac embryonnaire, dans lequel se forme l'albumen $(e d p)$, et où l'on voit les corpuscules $(c p) ; b$, écaille, à l'aisselle de laquelle s'est formée la fleur; 9 , fruit à demi formé ; 10 , le même, fendu en long ; $a$, disque charnu de couleur rouge, qui s'élève autour du fruit proprement dit; $e$, le germe; 11 , aiguille ou feuille de l'If avec sa coupe transversale; 12, aiguille du Sapin; 13, celle du Pin. 
albumen ou endosperme abondant. Au milieu de cet endosperme plusieurs cellules se distinguent par leur volume considérable; souvent groupées les unes près des autres, chacune est surmontée par une rosette de quatre petites cellules interposées entre elle et la membrane du sac embryonnaire. On désigne sous le nom de corpuscule chaque grande cellule accompagnée de sa rosette de quatre petites (fig. 128,I,c,b); chaque corpuscule est destiné à devenir un embryon. Il n'y a rien de plus dans le sac embryonnaire, ni vésicules synergiques, ni vésicules antipodes.

Suivant les espèces, un seul tube pollinique ou plusieurs pénètrent jusqu'au nucelle, dont l'accès est facile ; et là, ils restent stationnaires pendant un temps variable, tout en gardant leur entière activité, tandis que l'ovule achève d'arriver à maturité pour devenir apte à être fécondé.

Ainsi, dans les Conifères qui mûrissent leurs fruits en un an, cet état stationnaire a une durée de quelques semaines à quelques mois, tandis qu'elle est d'une année pour ceux qui, comme le Genévrier, le Pin sylvestre, emploient deux ans à mûrir leurs fruits.

Évolution de

L'ovule fécondé subit bientôt des cloisonnements formant 'ovule fécondé. plusieurs étages superposés de cellules. Le corps qui en résulte porte le nom de pré-embryon. Bientôt, en effet, sa petite masse va se diviser en deux parties distinctes, dont la supérieure constituera le suspenseur, tandis que l'inférieure sera l'embryon proprement dit (fig. 128, I, $d$ ).

Ce dernier en se développant allonge sa tigelle, autour de laquelle se voient souvent plus de deux et parfois un grand nombre de cotylédons (3 à 14), disposés en un seul verticille (fig. 135).

Chaque corpuscule devenant embryon, il en résulte qu'un même nucelle donne naissance à plusieurs embryons ; mais, comme dans les autres plantes phanérogrames, il ne se pro duit, en réalité, qu'un seul embryon susceptible de se transformer en graine, les autres s'atrophiant à un moment donné et servant à la nourriture de celui qui prend le dessus. L'endosperme contribue aussi au développement de ce dernier, qui, d'ailleurs, n'absorbe jamais complètement cette réserve 
nutritive, dont il reste toujours une notable portion dans la graine arrivée à sa maturité.

Modifications des autres parties de l'ovule. - Tandis que les parties profondes de l'ovule subissent les changements considérables qui ont été décrits, les parties extérieures se modifient également.

Des deux enveloppes de l'ovule (primine et secondine), l'interne se résorbe, et l'externe seule persiste, pour devenir le légument de la graine. Quand au funicule et au hile de l'ovule, ils deviennent le funicule et le hile de la graine.

Mais ces organes extérieurs et même certaines parties de la graine deviennent parfois le point de départ de pro-

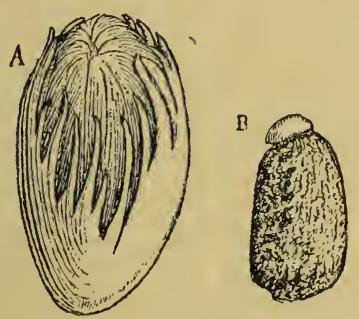

Fig. 130. - A, arille de la Muscade, découpé en bandelettes; $B$, arille en forme de caroncule, de la graine du Ricin. ductions singulières, d'excroissances, que l'on désigne sous le nom d'arilles. Leurs dimensions sont très variables; elles peuvent consister en une simple caroncule, comme dans le Ricin, ou acquérir un développement énorme, de façon à recouvrir entièrement la graine, comme cela a lieu pour la Muscade, où l'arille, connu sous le nom de macis, a la forme de longues bandelettes charnues de couleur jaune rougeâtre.

Hybrides; Métis. - On a vu plus haut, au sujet de la pollinisation, autrement dit, de la mise en contact du pollen avec le pistil, le rôle joué par les courants atmosphériques, les insectes, parfois même les oiseaux. On conçoit facilement que ces mêmes causes déterminent le transport du pollen de certaines fleurs sur d'autres d'espèce différente.

Et alors, de deux choses l'une, ou bien il ne se produira pas de fécondation, et telle est la règle dans la majorité des cas; ou bien il y aura fécondation, comme cela se rencontre 
quand les deux espèces sont, par leurs caractères, voisines l'une de l'autre et appartiennent à un même genre.

Les graines qui en résultent produisent ce qu'on appelle des hybrides.

Métis. Si la fécondation peut se produire entre fleurs d'espèces différentes, à plus forte raison peut-elle s'accomplir entre les diverses variétés d'une même espèce; on réserve plutôt le nom de métis aux individus obtenus par cette sorte de croisement.

Les horticulteurs, en imitant les procédés tout à fait fortuits de la nature, ont obtenu de la sorte un nombre infini de variétés. Malheureusement, elles ne donnent que rarement des graines, les hybrides, du moins, et il est nécessaire pour leur conserver leurs caractères, de les multiplier par greffe, boutures ou marcottes.

Les plus belles fleurs de nos jardins sont obtenues par les procédés qui viennent d'être indiqués; ainsi les Roses, les Jacinthes, les Pensées, les Tulipes, en sont des exemples bien connus. L'hybridation a, en effet, pour résultat, de donner des fleurs plus belles, mieux nuancées, plus hâtives, en même temps que la tige et les feuilles sont plus développées et que la plante est plus rustique.

Les variétés obtenues par hybridation ou métissage tiennent à la fois de l'un et de l'autre parents, non pas que les caractères des deux soient pour ainsi dire fondus ; ils sont plutôt juxtaposés; de façon que l'on peut distinguer tant ce qui appartient à l'un, que ce qui revient à l'autre.

Une conséquence de cette disposition, c'est qu'à un moment donné, surtout si on apporte quelque négligence dans la culture de ces variétés, il y a retour vers l'un ou l'autre des producteurs, les caractères de l'un d'eux prenant le dessus, tandis que ceux de l'autre disparaissent complètement. Il y a là comme une barrière posée par la nature pour empêcher la variation indéfinie, une sorte de force qui oblige la plante à revenir au type spécifique. 


\section{CHAPITRE XI}

\section{Fruit et Graine.}

Orıgine et parties constitutives du Fruit; Loges et cloisons. Parties accessoires du Fruit. Déhiscence. Classification des fruits. Constitution et structure de la Graine. Dissémination des fruits et des graines.

Origine et parties constitutives du Frutt. - Comme il a été dit au chapitre précédent, page 195, le fruit n'est autre chose que l'ovaire lui-même, qui subit d'importantes modifications après la fécondation de l'ovule, lequel, de son côté, se transforme en graine.

Dans l'étude du fruit, nous devons considérer successivement le péricarpe, qui dérive de la paroi de l'ovaire; les loges et les cloisons qu'il présente; le mode de déhiscence, quand celle-ci a lieu; enfin, les différentes sortes de fruits ou leur classification.

PÉricarpe. - Le péricarpe n'étant autre chose que la paroi de l'ovaire, nous en connaîtrions déjà la structure, s'il n'y survenait pas de modifications pendant la période de la maluration; mais l'épiderme extérieur ou épicarpe, le parenchyme intermédiaire ou sarcocarpe et l'épiderme interne ou endocarpe peuvent être le siège de changements importants, sur lesquels nous allons jeter un coup d'œil en prenant pour types un fruit sec, la noisette, et un fruit charnu, la pếche.

Dans le premier cas, le péricarpe, formé des deux épidermes interne et externe et du parenchyme intermédiaire confondus entre eux, devient une masse dure, résistante, par suite de l'épaississement des parois des cellules constitutives, 
qui s'incrustent de matière ligneuse, en même temps que leur cavité se rétrécit beaucoup; le péricarpe n'est autre chose, par conséquent, que la coquille du fruit.

Dans le second cas, les parties du péricarpe restent plus

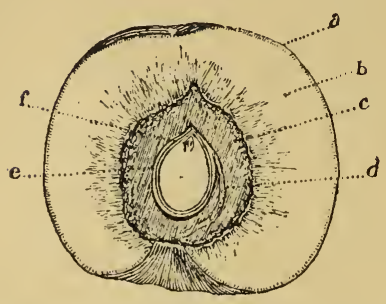

Fig. 131. - Fruit à péricarpe charnu (Pêche); $a$, épiderme; $b$, parenchyme ou sarcocarpe; $c$, endocarpe ou noyau; $d$, raphé; $e$, tégument de la graine; $f$, embryon. distinctes : l'épiderme extérieur s'épaissit, devient duveteux et constitue ce qu'on appelle la peau du fruit; le parenchyme prend un énorme accroissement, par suite de la multiplication et de l'agrandissement de ses cellules primitives, dont les parois restent fort minces et se remplissent de sucs savoureux. Quant aux vaisseaux qui parcouraient les parois de l'ovaire, on les retrouve disséminés comme de très fins filaments au milieu de la pulpe du fruit.

Ajoutons que, tandis que ces modifications anatomiques s'opèrent dans les tissus du fruit, il s'en passe d'autres de nature chimique; c'était d'abord de la fécule, du tannin, des acides, qui se trouvaient accumulés dans les cellules, et le fruit avant la maturité était fade et âcre ; mais par suite de la transformation de ces substances en glycose, le fruit est devenu doux et savoureux.

Quant aux couches les plus internes du parenchyme de la Pêche, jointes à l'épiderme interne, elles ont subi une toute autre modification. Elles ont pris, en effet, une consistance remarquable, leurs cellules ont épaissi leurs parois par accumulation de matière ligneuse et rétréci leur cavité pour former un tissu dont la dureté égale celle de la pierre, et qui a reçu le nom de noyau. C'est une modification analogue, qui se produit partiellement dans plusieurs espèces de poires, où l'on voit certaines cellules, par suite de l'épaississement et de l'incrustation de leurs parois, acquérir la dureté de la pierre, d'où le nom de poires pierreuses, qu'on leur donne. 
Loges et Cloisons. - La disposition des loges que présente l'intérieur des fruits, et celle des cloisons qui limitent les premières, rappellent le plus souvent la disposition qu'elles offraient dans l'ovaire; mais il y a d'assez nombreuses exceptions, le nombre des loges pouvant diminuer par avortement de certains carpelles. C'est ainsi que, tandis que l'ovaire du Noisetier a deux loges, celui du Ghêne, trois, et celui du Châtaignier, six, le fruit de tous ces arbres n'en a qu'une, est uniloculaire.

Il peut se faire, d'autres fois, que, par suite de la formation de cloisons surnuméraires, le nombre des loges se trouve augmenté.

Nous retrouvons dans les loges du fruit les placentas, avec la situation qu'ils occupaient dans l'ovaire, mais ayant souvent açquis un volume beaucoup plus considérable, au point qu'ils remplissent parfois toute la cavité de l'ovaire et forment une masse dans laquelle les graines sont enfoncées.

Parties accessolres du fruit. - Il arrive assez souvent que le pistil n'est pas la seule partie de la fleur qui prenne de l'accroissement après la fécondation. Le calice, comme on l'a déjà dit, est assez souvent dans ce cas, et forme autour du fruit une enveloppe plus ou moins complète, sèche ou charnue; le Groseiller du Cap (Physalis) offre un exemple bien connu de la première transformation; dans le Mûrier en arbre, il se gorge de sucs savoureux et englobe chacun des nombreux petits fruits dont l'ensemble constitue la mûre. D'autres fois, c'est l'espèce de coupe formée par la base du calice et de la corolle réunis, comme on le voit dans le cas des ovaires infères, le Rosier, par exemple, qui devient accrescente et charnue.

Enfin, le réceptacle lui-même peut en se développant considérablement contribuer à augmenter le volume et la saveur du fruit ; ainsi dans le Fraisier, c'est lui qui forme ce cône allongé autour duquel sont disposés les petits fruits nombreux dont l'ensemble constitue la Fraise; dans le Figuier, le réceptacle profondément concave porte dans sa 
cavité une multitude de petits fruits secs, et c'est lui-même, qui se gonflant de sucs savoureux, fait de la Figue le fruit excellent que l'on connaît.

Déhiscence des rnuits. - Arrivés à leur maturité, les fruits d'un grand nombre d'espèces s'ouvrent pour laissel échapper leurs graines, mais, le mode de déhiscence est très variable. D'autre part, un grand nombre de fruits ne sont pas déhiscents; il en est ainsi pour la plupart, de ceux qui sont charnus, comme la poire, la pomme, la pêche, et aussi de ceux qui sont secs el renferment une seule graine, comme le blé, l'orge, le seigle, etc.

La déhiscence peut se faire au moyen de pores (Pavot), par fente transversale (Mouron); mais, dans la règle, elle a lieu par fente longitudinale. Les parties de l'enveloppe du fruit, séparées par suite de la déhiscence, portent le nom de valves.

Cette déhiscence se fait parfois très brusquement, avec une grande force, de façon à projeter les semences au loin; la Balsamine en offre un exemple des plus connus. Dans ce cas, elle est due à la présence d'une couche de cellules aplaties, à contours variables, qui traversent le péricarpe dans toute son épaisseur et qui, lorsqu'elles viennent à perdre une certaine quantité d'eau, se resserrent, reviennent sur elles-mêmes et déterminent la fente du péricarpe dans toute sa longueur.

Différents modes de délisiscence.

Les différents modes de déhiscence, qui servent à caractériser les diverses sortes de fruits, seront exposés dans la classification ci-dessous; nous allons seulement définir ici trois grands modes de déhiscence, que l'on observe dans les fruits résultant de la réunion de plusieurs carpelles soudés entre eux.

Dans de semblables fruits, on doit d'abord distinguer des sutures ventrales, formées par l'accolement des bords de chaque feuille carpellaire repliée, et qui occupent le centre du fruit; des sutures dorsales, répondant à la nervure moyenne de chaque feuille carpellaire et situées à la partie extérieure; des sulures pariétales, formant, à la surface du fruit, des lignes enfoncées là où s'arrête l'accolement de deux 
carpelles voisins. Enfin, si les carpelles au lieu de se replier en dedans vers le centre du fruit, se soudent l'un à l'autre, bord à bord, de façon à former une cavité unique, les lignes suivant lesquelles ils sont accolés 'sont dites sutures marginales.

Or, la déhiscence se fait toujours suivant l'une ou l'autre de ces sutures.

Si elle a lieu suivant les sutures dorsales, chacune des valves qui en résultent offre, fixée au Déliscences : loculicide;

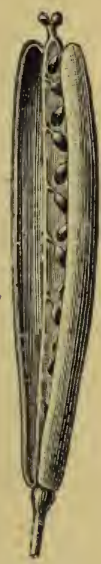

Fig. 132. - Exemple de fruit sec à déhiscence longitudinale (Silique). milieu de sa paroi interne, une cloison résultant de l'accolement des faces latérales adjacentes de deux carpelles voisins, et la déhiscence est dite 10culicide; tel est le cas de la Tulipe.

Si elle a lieu par les sutures pariétales, la cloison formée par l'accolement des parois adjacentes des deux carpelles voisins se dédouble, et chacun de ceux-ci se trouve par là même isolé ; la déhiscence est alors appelée septicide; le Ricin en offre un exemple.

$\mathrm{Si}$ la déhiscence a lieu encore au niveau des sutures pariétales, mais de façon que les cloisons restent intactes au lieu de se dédoubler, la paroi externe seule du fruit se sépare en autant de valves qu'il y a de carpelles constituants, et la déhiscence est dite septifrage; on l'observe dans le $D a$ tura ou Pomme-épineuse.

Classification des fruits. - D'après le nombre èt les relations des carpelles qui entrent dans la composition des fruits, on peut répartir ceux-ci en quatre grands groupes :

Fruits simples, formés par un seul carpelle ;

Fruits multiples ou polycarpés, formés par plusieurs carpelles distincts ;

Fruits soudés ou syncarpés, résultant de la réunion de plusieurs carpelles accolés provenant d'une même fleur.

septifrage.

septicide ; 
Fruits composés ou synanthocarpés, dus à la soudure de plusieurs carpelles appartenant à des fleurs différentes.

Dans le tableau ci-joint sont résumés les caractères des principaux fruits qui méritent un nom particulier.

1. Fruits secs.

Indéhiscents.

Akène, fruit monosperme, péricarpe distinct du tégument de la graine (Chardon, Oseille).

Caryopse, akène dont le péricarpe est soudé au tégument de la graine (Graminées).

Samare, akène pourvu d'appendices en forme d'aile (Frêne, Orme).

Capsule, fruit sec déhiscent ne rentrant pas dans les espèces suivantes (Tulipe) ; si la déhiscence, au lieu d'être longitudinale, se fait par de petits trous, on dit la capsule poricide (Pavot).

Follicule, formé par un carpelle unique dont les bords accolés se disjoignent à la maturité ( $\mathrm{Pi}-$ voine).

En long. Gousse ou Légume, consiste en un carpelle unique, se séparant à la maturité en deux valves par une double fente, l'une ventrale, l'autre dorsale (Légumineuses).

Silique, sorte de gousse qui s'ouvrant par quatre fentes, deux ventrales et deux dorsales, ayant pour effet d'isoler du reste du fruit les placentas développés en une fausse cloison qui porte les graines (fig. 132). En travers. $\left\{\begin{array}{l}\text { Pyxide, fruit simple, unilocu- } \\ \text { laire, se divisant en deux valses } \\ \text { superposées par une ligne de } \\ \text { déhiscence circulaire (Mouron, } \\ \text { Jusquiame). }\end{array}\right.$

2. Fruits charnus.

Indéhiscents. Baie, pas de noyau (Raisin, Groseille).

Déhiscents. Capsule charnue (Balsamine, Marronnier).

$3^{\circ}$ Fruits mi-partie secs et charnus.

Drupe, fruit charnu à l'extérieur, renfermant Indéhiscents. $\quad \begin{aligned} & \text { un noyau dur uniloculaire (Cerisier, Prunier, } \\ & \text { Pêcher). }\end{aligned}$ 
Déhiscents. $\quad\left\{\begin{array}{c}\text { Capsule drupacée ou noix, diffère du fruit } \\ \text { précédent par son péricarpe plus coriace, } \\ \text { (Amandier, Noyer, Cocotier). }\end{array}\right.$

Gonstitution et structure de la Graine.

Graine; Ses enveloppes; L'Embryon. - L'ovule, en passant à l'état de graine sous l'influence de la fécondation et en arrivan ı peu à peu à la maturité, éprouve, comme on l'a vu précédemment, des changements considérables, le plus souvent par augmentation du nombre de ses parties constituantes, plus rarement par leur réduction. Ainsi, tandis que dans la règle l'ovule se compose d'une petite masse centrale, le nucelle, et de deux enveloppes, il peut arriver qu'à la maturité, par suite du dédoublement de l'une d'elles, il en existe trois ou même quatre, comme cela se voit pour le Ricin. Par contre, il peut se faire que l'enveloppe de la graine se compose d'un tégument unique.

Le tégument de la graine ou épisperme (fig. 131,e), peut être sec et membraneux, comme darıs le Pêcher, le NoiseTégument de la graine. tier, le Noyer, ou dur et épais, comme dans le Pin, ou bien charnu, ainsi que le montre la Grenade.

Sa surface est ordinairement lisse, mais assez souvent marquée de dessins en relief, de lignes sinueuses, de côtes ou de ponctuations. Les cellules qui entrent dans la constitution de l'épisperme offrent une grande variété au point de vue de leur dimension, de leur forme et du nombre de leurs couches superposées. Souvent, les plus superficielles sont transformées en poils, qui peuvent acquérir une grande dimension, comme dans le Cotonnier, où elles constituent le coton; parfois elles sont groupées seulement en certains points, sous forme d'aigretles, qui servent à la dissémination des graines. Enfin, dans l'épaisseur du tégument se ramifient des faisceaux libéro-ligneux.

La partie renfermée sous le tégument de la graine est l'amande(fig.131), qui se compose de l'embryon seul ou accom-

Amande. 
pagné d'un endosperme ou d'un périsperme, ou même des deux à la fois.

Embryon. L'embryon (fig. 126), est formé d'un petit cylindre, la tigelle (st), présentant une extrémité conique, la radicule $(w)$, tandis que l'autre extrémité est très renflée, ovoïde ou aplatie, souvent facile à séparer en deux parties, les cotylédons (fig. 135, I, c), entre lesquels se voit un très petit cône végétatif qui surmonte la tigelle et qui présente souvent un bourgeon foliaire microscopique, la gemmule (fig. 126, k). Dans les Monocotylédones (fig. 126), il n'y a qu'un cotylédon $(s s, s c)$, qui offre sur un de ses bords une petite fente au fond de laquelle est logé le cône végétatif de la tigelle. Chez les Conifères, il y en a souvent plusieurs.

Les cotylédons sont épais et charnus, ou minces, membraneux et différemment repliés et plissés selon, que l'albumen a disparu ou qu'il a au contraire persisté. Lorsque l'albumen existe, l'embryon y est ordinairement plongé, mais de façon, en tout cas, ,à être très rapproché du micropyle de la graine, vers lequel se trouve toujours dirigée la radicule (fig. 135, I, y); quelquefois, au contraire, il est situé en dehors de cette réserve nutritive, autour de laquelle s'étale un des cotylédons.

Agents de

Dissémination des fruits et des graines. - Les agents la dissemination: de dissémination des fruits et des graines sont fort variables, comme on va le voir. Un cerlain nombre de fruits sont le vent; pourvus d'organes légers, grâce auxquels ils peuvent être soulevés par le vent et emportés à des distances souvent considérables. Ainsi ceux des Chardons, des Bluets, des Salsifis, des Pissenlits (fig. 133), portent des aigrettes de forme variable, sortes de parachutes qui leur permettent de flotter en l'air. Toutes les espèces de la famille des Composées sont ainsi pourvues de semblables organes de dissémination. Dans d'autres plantes, c'est la graine elle-même qui offre une particularité analogue; telle est celle du Saule. D'autres fois, au lieu d'une aigrette, le fruit est pourvu d'un organe étalé en manière d'aile, et qui sert à jouer un rôle identique : ceux de l'Érable et de l'Orme, que l'on voit 
tournoyer au vent, nous en offrent des exemples bien connus.

Un autre ordre de causes de dissémination réside, non plus dans les courants aériens, mais dans les cours d'eau, qui transportent à des distances parfois considérables cer-

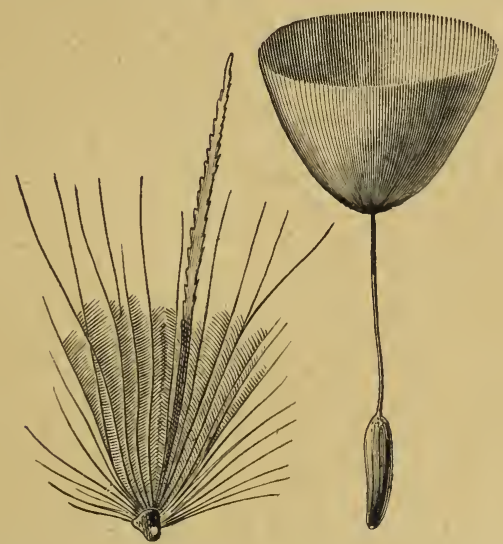

Fig. 133. - Organes de dissémination; à gauche, un fruit de Graminée (Pennise(um); à droite, une graine de Pissenlit.

tains fruits ou graines, lesquels peuvent résister, souvent pendant bien des jours, sans altération, à l'action de l'eau, et être conduits au loin par les courants marins ou les fleuves.

Les animaux, surtout, constituent une cause active de les animaux; l'extension géographique des plantes. Ainsi, un certain nombre de fruits, qui ont leur surface hérissée d'aspérités leur permettant de s'accrocher aux poils et surtout à la laine des animaux, se trouvent fortuitement transportés d'un pays dans l'autre, en même temps que les toisons de ces animaux auxquels ils se sont fixés. C'est ainsi que jadis à Port-Juvénal, près de Montpellier, et aujourd'hui aux environs du port de Marseille, par suite de grands arrivages de laines de différents pays, on a pu observer l'existence d'une flore toute particulière et très changeante suivant les temps, laquelle a pour origine la cause qui vient d'être signalée.

D'autre part, différents animaux, des oiseaux surtout, transportent avec leur bec les fruits ou les graines de cer- 
taines plantes et les laissent tomber çà et là; c'est ainsi qu'une plante parasite bien connue, le Gui, passe d'un arbre à l'autre; ou bien, ces animaux mangent les fruits, dont les noyaux résistants traversent leur tube digestif sans que l'amande en soit altérée. On explique par ce moyen de dissémination l'existence de ces végétations nombreuses qui couronnent le sommet des vieux monuments mème les plus élevés. G'est en grande partie à cette cause que l'on doit attribuer la richesse de la flore que l'on est surpris d'observer à Rome, sur les ruines du Colisée, et qui ne comprend pas moins de 260 espèces.

l'homme. Enfin, l'homme lui-même est de tous l'agent le plus actif de la dissémination des plantes, soit volontairement, soit d'une façon fortuite. Ainsi, les migrations des peuples, les déplacements de tribus nomades ont constamment pour conséquence l'apparition d'espèces végétales dans des lieux où elles étaient complètement inconnues auparavant. Ia Pomme-épineuse (Datura stramónium), par exemple, qui est commune au bord des chemins, dans beaucoup de parties de notre pays, nous a été apportée de l'Inde par les Bohémiens. Le séjour des Russes et des Cosaques en France, en 1815, a été suivi de l'apparition de végétaux qui ne croissaient auparavant que sur les bords du Don. Le célèbre voyageur Auguste Saint-Hilaire rapporte que nombre de nos plantes indigènes se sont naturalisées au Brésil, par suite des rapports commerciaux que nous avons eus avec ce pays ; cilons, entre autres, la Violette, la Bourrache, le Fenouil, plusieurs Géraniums, l'Avoine, des Mauves, etc. Le Chardon-Marie, si connu chez nous, abonde dans les plaines du Rio de la Plata et de l'Uruguay.

D'autres plantes, enfin, sont importées avec intention formelle en raison des avantages que l'on peut retirer de leur culture au point de vue industriel ou alimentaire; citons entre autres, comme l'une des plus célèbres, le Caféier, originaire de l'Arabie et qui, transporté aux Antilles, il y a un peu moins de deux siècles, fournit presque tout le café consommé en Europe. 


\section{GHAPITRE XII}

\section{Germination.}

La vie latente de la graine. Conditions nécessaires à la germination. Phénomènes morphologiques 'et phénomènes chimiques de la germination.

La vie latente de la graine. - La graine est un être vivant; les manifestations vitales, quoique très faibles, n'y sont pas entièrement suspendues et se traduisent encore par une légère absorption d'oxygène et un faible dégagement d'acide carbonique. La vie peut ainsi sommeiller dans la graine pendant des années et parfois même pendant des siècles, pour se réveiller ensuite. C'est ainsi qu'après des remaniements de terrain, des travaux de terrassement, on voit apparaître dans un pays des plantes depuis longtemps inconnues, dont les graines, enfouies pendant un grand nombre d'années, se sont mises à germer aussitôt qu'elles ont été placées dans des conditions convenables. Bien plus, on a pu faire lever des graines trouvées dans des tombeaux gallo-romains, et même, dit-on, des graines venant des tombeaux des anciens Egyptiens et remontant, par conséquent, à plusieurs milliers d'années.

Conditions nécessaires a la germination. - Pour être susceptible de germer, il faut que la graine soit bien conConditions intrinsèques. formée, qu'elle soit bonne, comme on dit généralement. Il arrive assez souvent qu'une graine, tout en ayant son volume normal, a quelqu'une de ses parties essentielles avortée; en général, ces mauvaises graines se reconnaissent assez facilement à la particularité qu'elles présentent de 
surnager quand on les jette dans l'eau, contrairement aux bonnes qui, plus lourdes, tombent bientôt au fond.

Une autre condition indispensable à la germination, c'est que la graine soit mûre, état qui coïncide, en général, avec la maturité des parties extérieures du fruit, mais qui peut la précéder, comme cela a liẹu dans un grand nombre de plantes de la famille des Légumineuses, ou la suivre, comme c'est le cas de celles du Pêcher, du Rosier, etc., qui doivent attendre souvent deux années avant de pouvoir entrer en germination.

De plus, les graines ne doivent pas être trop vieilles. La durée de la faculté germinative est d'ailleurs très variable, car si quelques-unes peuvent germer au bout de plusieurs centaines d'annćes, comme on l'a dit un peu plus haut, il en est qui doivent être semées dans l'année même de leur récolte, si l'on veut qu'elles se développent. Ainsi les graines qui, comme celles du Café, des Ombellifères, ont un albumen corné, perdent promptement par la dessication leur faculté germinative. Les graines oléagineuses la conservent plus longtemps, mais l'huile qu'elles contiennent venant à rancir, elles ne sont plus bonnes au bout de quelques années. Les graines amylacées ou farineuses sont celles qui, de toutes, résistent le plus longtemps.

Les circonstances extérieures peuvent être nuisibles aux graines à des degrés divers : le froid est, en général, sans action sur elles, quand elles sont bien sèches; la chaleur est égálement bien supportée, du moins quand elle n'est pas humide; ainsi le Blé, le Maïs supportent sans altération une température de $100^{\circ}$, à l'air sec; mais l'eau chauffée à $50^{\circ}$ environ les tue promptement.

Conditions extrinsèques.

Trois conditions extérieures sont indispensables à la germination : la chaleur, l'humidité, l'oxygène.

La température nécessaire varie suivant les espèces; quelques-unes germent à 0 o, la plupart entre $20^{\circ}$ et $25^{\circ}$.

L'humidité est tellement indispensable, que c'est à son absence que l'on doil la conservation presque indéfinie de certaines semences; ainsi des graines de Tabac ou autres, conservées pendant deux siècles dans des herbiers 
maintenus á l'abri de l'humidité, n'ont germé que quand on les a mises au contact de l'eau. Des graines placées dans une terre bien sèche sont incapables de germer, bien que la température soit suffisamment élévée.

L'oxygène, enfin, doit être fourni aux semences en suffisante quantité, sous peine de ne pas les voir se développer. Ainsi, plongées dans un vase hermétiquement clos, renfermant de l'acide carbonique ou del'azote, avec un peu d'eau, et en présence d'une température convenable, elles ne germent pas; mais remplace-t-on ces gaz par de l'air, elles se mettent bientôt à lever. De même, si on place des graines dans de l'eau privée d'oxygène par la distillation ou l'ébullition, elles n’éprouvent aucun changement. Si l'air ou l'oxygène se trouve à une pression considérable, 5 à 6 atmosphères, la germination ne se fait pas davantage. Il en est de même si l'on met les graines en contact avec des vapeurs anesthésiques, telles que celles du chloroforme ou de l'éther.

On a remarqué que le chlore, le brome, l'iode favorisent la germination des graines même très vieilles ou en partie altérées. Ce résultat paraît dépendre du dégagement, sous l'action de ces agents chimiques, d'une certaine quantité d'oxygène naissant, dû à une décomposition de l'eau du sol.

Enfin, comme circonstances secondaires, on doit ajouter que la lumière et surtout l'électricité paraissent favoriser notablement la germination.

Phénomènes morphologiques de la germination. - On peut établir, comme règle, quatre phases dans la germination de la graine ou la transformation de l'embryon en plantule. (Voy. fig. 135.)

La radicule s'allonge et sort au niveau du micropyle, en produisant une déchirure des tissus de l'enveloppe ramollis et distendus par le gonflement de l'amande. Elle se recourbe aussitôt vers le sol $(A, B)$, en vertu de la force connue sous le nom de géotropisme (1). (Voy. ch. xiv, p. 250.)

(1) $\Gamma \tilde{\eta}$, terre, $\tau \rho \varepsilon ́ \pi \varepsilon เ v$, tourner; c'est-à-dire qui se dirige vers la terre. 
Peu après, la tigelle s'allonge à son tour en se dressant verticalement en haut, dans la prolongation de l'axe de la racine; elle entraîne dans son mouvement les cotylé-

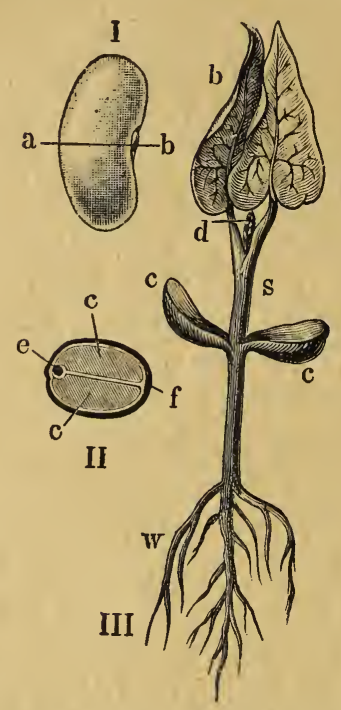

Fig. 131. - Germination du Haricot ; I, la graine entière; $a b$, ligne suivant laquelle elle est coupéc pour en montrer la structure, comme on la voit en II ; $c, c$, les cotylédons ; $e$, embryon ; $f$, tégument séminal; III, la plantule ; $c, c$, cotylédons; $s$, la jeune tige; $d$, cône végétatif; $b$, feuilles séminales; $w$, racines. dons, qui la surmontent, d'où son nom de tige hypocotylée (fig. 135, $h c$ ).

Ensuite, les cotylédons se gonflent de plus en plus, de façon à faire éclater les enveloppes de la graine; ils s'écartent l'un de l'autre et s'épanouissent en constituant les deux premières feuilles vertes de la plante, appelées feuilles séminales (fig. 134, b).

Plus tard, enfin, l'extrémité de la tige ou cône végétatif, nu ou enveloppé de la gemmule ou bourgeon terminal $(d)$, s'élève au-dessus des cotylédons, pour former la tige épicotylée (fig. 134,s).

Il y a de nombreuses exceptions à cette marche classique du développement de la plantule; ainsi, les cotylédons, au lieu de s'épanouir dans l'atmosphère sous forme de feuilles vertes, restent souvent enfouis dans le sol, et après le développement de la radicule, la gemmule seule s'élève au-dessus de la terre, en se faisant jour par l'orifice de sortie de la radicule. Quand les cotylédons restent ainsi cachés, on les dit hypogés, tandis que dans le premier cas, ils méritent le nom d'épigès.

Phénomènes chimiques de la germination. - Pendant sa germination, la plantule absorbe de l'oxygène, dégage de l'acide carbonique et émet de la vapeur d'eau; en même 
temps, elle perd de son poids en matières solides. Cette perte a pour objet le carbone, l'hydrogène, l'oxygène, mais

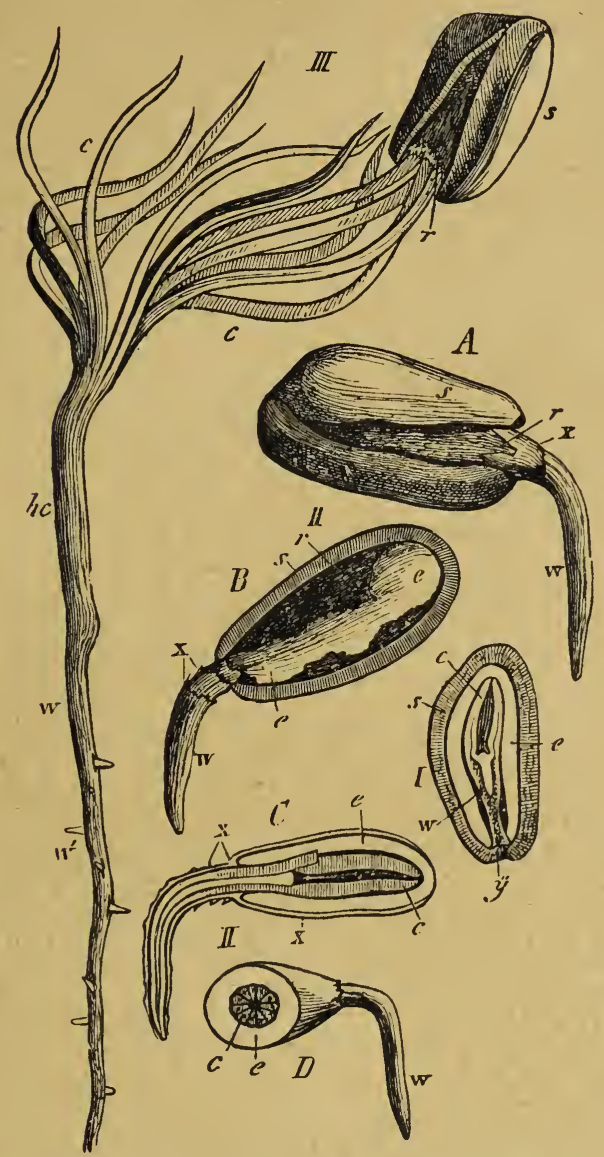

Fig. 135. - Germination d'une graine de Conifère, Pin (Pinus picea); I, la graine fendue en long; II, début de la germination; $A$, enveloppe séminale $(s)$, déchirćc par le gonflement des parties internes et laissant sortir la racine; $B$, la même, avec une moitié de l'enveloppe enlevée et montrant l'endosperme $(e) ; C$, coupe longitudinale; $D$, coupe transversale de la même, montrant de nombreux cotylédons; III, fin de la germination, tout l'endosperme étant épuisé; les cotylédons achèvent de sortir des enveloppes de la graine; $-c$, cotylédons; $e$, endosperme ou albumen; $h c$, tigelle liypocotylée; $w$, racine terminale; $w$, radicelles $; r$, couclie interne rouge du tégument; $s$, couche externe ligneuse du même; $x$, sac embryonnaire, que l'on voit déchiré en $x(B)$, sous la poussée de la radicule ; $y$, micropyle. 
non l'azote ni les matières minérales. Le dégagement d'hydrogène et d'oxygène se faisant dans les proportions qui

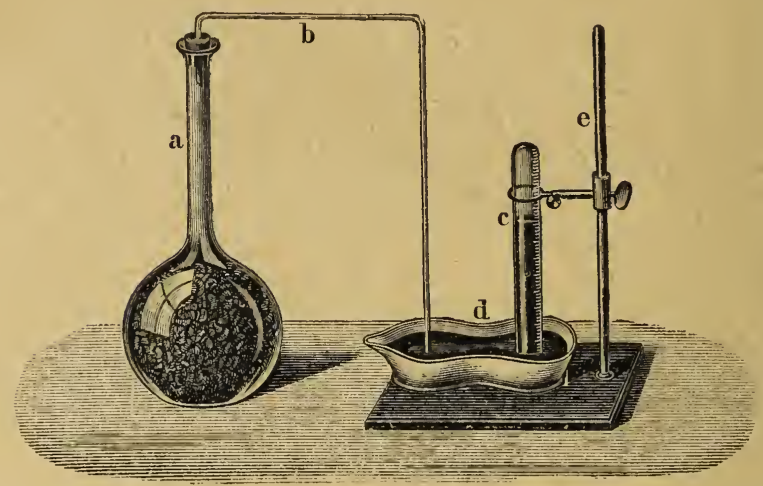

Fig. 136. - Appareil destiné à recueillir les gaz et vapeurs qui se dégagent lors de la germination des graines. Des semences de Fève un peu gonflées par l'eau sont placées dans un ballon de verre $(a)$, d'où part un tube $(b)$, qui aboutit à une éprouvette graduée $(c)$, pleine de mercure et maintenue par le montant $(e)$ dans une cuvette à mercure $(d)$. Au bout d'un certain temps on analyse les gaz qui se sont accumulés dans l'éprouvette en refoulant le mercure, et on pìse, après les avoir desséchées, les semences qui sont devenues des plantules, afin de comparer leur poids à celui des semences sèches préalablement pesées.

constituent l'eau, on peut dire, en résumé, qu'il y a perte d'eau et de carbone.

En outre, il y a dégagement de chaleur, comme on peut le démontrer en engageant un thermomètre dans une masse de graines en voie de germination, tandis qu'un autre thermomètre indique par comparaison, la température de l'air ambiant. (Voy. Chap. de la Respiration.)

Emploi des réserves nutritives.

En même temps, les aliments de réserve accumulés dans la graine se digèrent et se transforment; ainsi les substances amylacées se changent en glycose, en passant par l'état de dextrine et de maltose; il en est de même des matières sucrées, qui se dédoublent d'abord en glycose et lévulose. Finalement, toutes les substances sucrées ou anylacées sont transformées en glycose soluble et assimilable. Cette opération chimique se fait sous l'influence d'un ferment spécial, la diastase, due à la transformation d'une certaine quantité de substances albuminoïdes. 
Si la réserve nutritive se compose de matières grasses, celles-ci se saponifient, c'est-à-dire, qu'elles se dédoublent en glycérine, qui est aussitôt absorbée et assimilée, et en acides gras, qui se dédoublent et se transforment finalement en hydrates de carbone, lesquels deviennent, pour la plus grande partie, de l'amidon ou fécule.

Quant aux aliments albuminoïdes de réserve, ils s'hydratent et se transforment en peptones, puis vont s'accumuler dans les cellules végétales surtout sous forme d'asparagine, laquelle disparaîtra quand la matière verte fera son apparition (1).

(1) L'asparagine, dont la formule est $\mathrm{C}^{8} \mathrm{H}^{8} \mathrm{Az}^{2} 0^{6}$, est une substance d'une grande importance dans l'économie de la plante. Elle se forme constamment, mais se détruit au fur et à mesure de sa production, ou plutôt se transforme, en se combinant avec des composés ternaires, pour constituer des composés quaternaires ou albuminoïdes. Elle ne s'accumule dans les organes que dans le cas où les substances ternaires ne lui sont pas fournies en assez grande abondance pour pouvoir donner lieu aux combinaisons nouvelles qui viennent d'être indiquées. La formation de l'amidon, substance ternaire, étant liée à l'existence de la matière verte ou chorophylle, on comprend pourquoi l'asparagine disparait quand cette dernière se montre. 


\section{CHAPITRE XIII}

Reproduction et Formes alternantes des Gryptogames. Parasitisme.

Modes de reproduction des Cryptogames.Reproduction asexuée par Spores et par Rajeunissement cellulaire. Reproduction sexuée : Conjugation; Fécondation. - Formes alternantes chez les Mousses, les Prêles, les Fougères, les Lycopodiacées, les Rhizocarpées et les Champignons. - Alternance de générations chez les Phanérogames. - Parasitisue. Exemples pris chez les Phanérogames et les Cryptogames ; Cryptogames épiphytes et Cryptogames épizoïques.

DifféRents modes de reproduction chez les CryptoGaMes. - Les Cryptogames, qui occupent les degrés inférieurs du règne végétal, pour être la plupart de faible taille, souvent même microscopiques, n'en ont pas moins un rôle considérable à jouer dans la nature, leur nombre immense venant compenser l'exiguité de leurs dimensions.

Tantôt leur structure est tellement simple que les seuls éléments que l'on y rencontre sont des cellules; tantôt leur organisation, un peu plus compliquée, montre une différenciation des éléments anatomiques, dont un certain nombre affectent la forme de vaisseaux, d'où la distinction des Cryptogames cellulaires et des Cryptogames vasculaires. Les premiers comprennent les Algues, les Champignons, les Lichens et les Mousses; les scconds, les Prêles, les Fougères, les Lycopodiacées et quelques autres groupes.

Un des caractères les plus remarquables des Cryptogames, c'est l'absence apparente des fleurs. Rien, chez eux, en effet, qui rappelle ces riches coulcurs, ce parfum des organes floraux que nous offrent les Phanérogames. Aussi, 
pendant longtemps a-t-on cru qu'ils en étaient dépourvus, et leur mode de reproduction restait un mystère, d'où leur nom même de Gryptogames (xpútròs, caché, ráuos, union). Du reste, aujourd'hui encore, pour un certain nombre d'entre eux, on ne sait que d'une façon incomplète cornment s'opère la reproduction.

Les organes essentiels des fleurs y existent néanmoins, mais petits, peu apparents, coinme cachés; malgré ces conditions, en apparence défavorables, leur reproduction est assurée par des moyens beaucoup plus variés que celle des plantes phanérogames; en outre, le cycle du développement d'un très grand nombre d'entre eux est bien plus compliqué.

Les différents procédés que l'on rencontre dans la multiplication naturelle de ces végétaux se réfèrent à l'un ou l'autre de ces deux grands modes : la reproduction asexuée et la reproduction sexuée. Dans cette dernière, une plante donne naissance à une autre, à condition qu'il y ait préalablement union intime d'élérnents de nature différente, formés par des organes spéciaux portés soil par deux individus distincts, soit par le même sujet. Dans le premier cas, au contraire, la naissance de la nouvelle plante n'est précédée, comme nous allons le voir d'abord, par rien de semblable.

Reproduction asexuée. - Les procédés employés en pareil cas par la nature sont très variés. Les Mousses, par exemple, peuvent se multiplier par stolons, c'est-à-dire au moyen de rameaux partis de la base de la tige et s'enracinant au contact du sol humide, comme nous le voyons dans certaines plantes phanérogames, notamment le Fraisier; par bourgeons, qui se détachent de la tige et peuvent se fixer au sol en produisant des racines et une tige, ou bien rester flottants dans certains liquides qui leur conviennent, comme font un certain nombre de ferments, entre autres celui de la levure de bière ou Saccharomyces cerevisice (fig. 137), par propagules, petits corps arrondis ou fusiformes portés par la tige ou les feuilles, et susceptibles de végéter par eux-mêmes ; par bulbilles, sortes de bourgeons adven- 
tifs qui se développent sur les poils radicaux de ces petites plantes.

Mais deux modes particuliers de reproduction asexuée

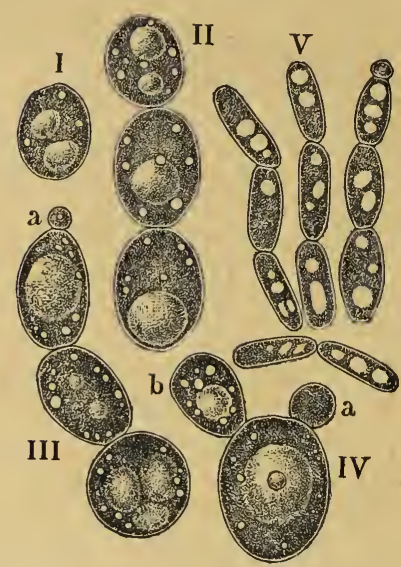

Fig. 137. - Levure de bière (Saccharomyces cercoisio); I, II, cellules isolées ou réunies; III, cellules en voie de bourgeonnement $(a)$; IV, cellule portant deux bourgeons, $a, b ; \mathrm{V}$, levure ou ferment du vinaigre (Saccharomyces mycoderma). méritent plus spécialement d'attirer l'attention; ce sont la formation des spores et le rajeunissement cellulaire.

$1^{0}$ Formation asexuée des spores. - Les Champignons vont nous servir de type pour l'étude de la formation des spores ou semences par voie asexuée. On doit savoir d'abord que ce qu'on appelle le Champignon, dans le langage vulgaire, n'est, en réalité, qu'une partie du végétal, à savoir, l'ensemble de la fructification; tandis que la plante proprement dite, la partie végétante, en un mot, est sous forme de filaments très tenus, rampants sous le sol, et dont l'ensemble porte le nom de mycélium.

Dans les Agarics, tels que Champignon de couche ou Champignon rose, par exemple, et dans bien d'autres genres, de la partie inférieure de l'espèce de chapeau, si variable dans sa forme, que tout le monde connaît, pendent des lamelles, des pointes ou des tubes serrés les uns contre les autres et dont l'ensemble constitue l'hyménium (ย̣uñv, membrane). Chacun de ces prolongements ou lamelles porte perpendiculairement à sa surface de microscopiques colonnettes ou basides (fig. 138), dont un grand nombre sont terminées par de petits renflements, qui ne sont autres que des spores. Gelles-ci, sont en quantité prodigieuse, et peuvent donner naissance à autant d'individus nouveaux, c'est-à-dire de 
mycéliums, qui, à un moment donné, fructifieront à leur tour et produiront de nouvelles spores.

Les dispositions secondaires présentées par les organes

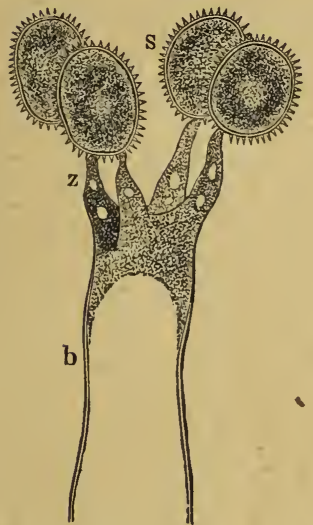

Fig. 138.

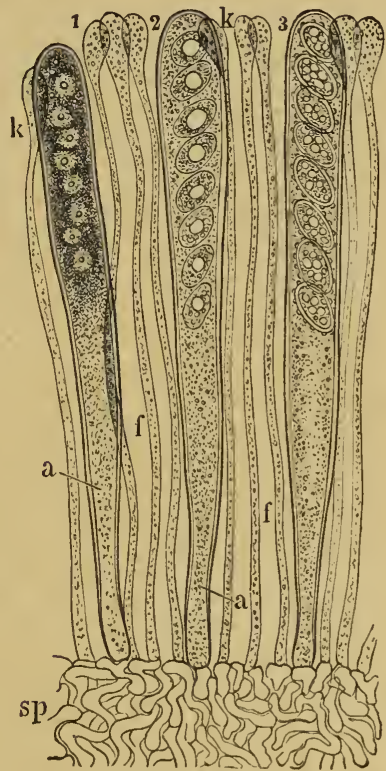

Fig. 139.

Fig. 138. - Une baside $(b)$, arec (quatre spores $(s)$ portées par autant de pédicelles ou stérigmates $(z)$.

Fig. 139. - Formation des spores dans des cavités eloses, en forme de tubes, d'un Champignon du groupe des Discomycètes (Pesizu aurantium). Petite portion de l'hyménium très grossie; $1,2,3$, cavités allongées ou asques contenant les spores; $a$, leur partie inférieure dépourvue de spores; $f, k$, sorte de poils ou paraphyses qui les séparent; $s p$, tissu sous-hyménial.

qui portent les spores sont loin d'être toujours les mêmes. La fig. 139 montre comment les choses se passent chez les Discomycètes, auxquels appartient la Morille. Chez les Gastéromycètes, ils sont renfermés dans une sorte de sac volumineux divisé en un grand nombre de chambres par des cloisons portant une énorme quantité de spores. Quand celles-ci sont mûres, le sac s'ouvre à sa partie supérieure et la moindre pression les fait sortir sous forme d'une lé- 
gère fumée, comme ont le voit dans la vulgaire Vesse-deloup (Lycoperdon bovista).

Le mode de reproduction qui vient d'être exposé est très général dans la classe des Champignons.

Les Truffes elles-mêmes, dont la nature a été pendant longtemps méconnue, se multiplient également par le moyen de spores renfermées au milieu de leur tissu dans de petites

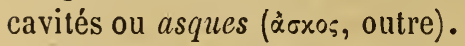

Cependant, quelques groupes, parmi les Champignons les plus inférieurs, présentent des faits de génération sexuée. De plus, on verra bientôt les phases compliquées par lesquelles passent certaines espèces pour arriver à former leurs spores reproductrices.

Dans les Lichens, qui encroûtent le tronc des arbres, ou qui s'étalent à la surface des pierres, des rochers, sur les vieux murs, sur nos toits ou sur le sol, les phénomènes de la reproduction sont identiques, dans leurs traits fondamentaux, à ceux des Champignons. Cela ne surprendra pas, quand on saura que ces êtres ne sont pas autre chose que des Champignons parasites d'Algues, ou, si l'on veut, que le Lichen résulte de l'association intime d'un Champignon avec une Algue, et qu'il est possible de reconnaître tant ce qui appartient à l'un, que ce qui fait partie de l'autre.

$2^{\circ}$ Rajeunissement cellulaire. - Voici en quoi consiste l'autre mode de reproduction asexuée, que l'on désigne sous le nom de rajeunissement cellulaire. Dans une cellule d'Algue filamenteuse, une Vauchérie, par exemple, le contenu protoplasmique se divise sous forme de deux petites masses, l'une plus condensée à la partie supérieure de la cavité cellulaire, l'autre un peu moins, à la partie inférieure. Entre les deux se trouve un espace plus clair rempli de suc cellulaire. Bientôt la petite masse protoplasmique supérieure traverse la paroi même de la cellule en se rétrécissant pour passer à travers une étroite déchirure, puis, une fois sortie, prend la forme d'un petit corps ovoïde, pourvu d'une partie plus aiguë ou rostre; il présente, en outre, des cils vibratiles et se meut dans l'eau avec agi-

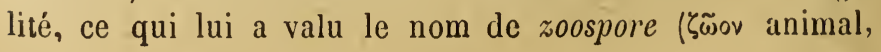


$\sigma \pi \circ \alpha^{\prime}$, graine). Bientôt, cette zoospore se fixe et devient le point de départ d'un nouvel individu semblable à celui qui lui a donné naissance.

Rephoduction sexuÉE. - Ici, encore, nous trouvons deux modes principaux de multiplication, dont l'un porte le nom de conjugation et l'autre celui de fécondation.

10 Conjugation. -- Une Algue va encore nous servir d'exemple pour la repro-
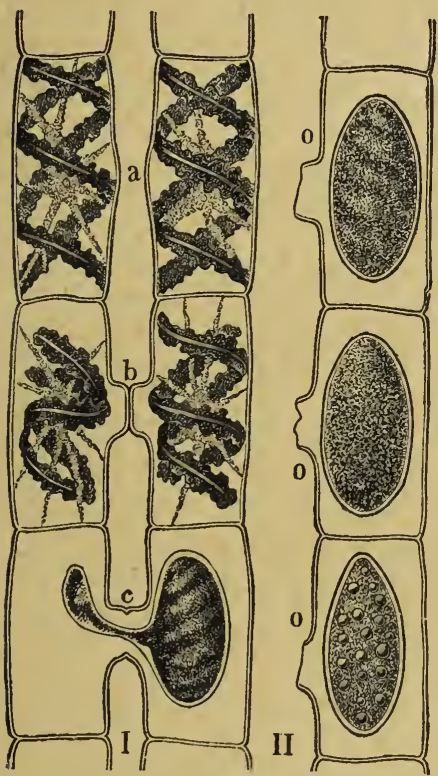

Fig. 140. - Reproduction d'une Algue, pary'conjugation (Spirogyra); I, deux filaments placés vis-à-vis l'un de l'autre et montrant les progrès de la conjugation en $a, b, c$; II, formation de la zygospore, en allant de haut en bas $(0)$. duction par conjugation. Les Spirogyres sont des Algues de nos fossés, formées de cellules mises bout à bout, dans lesquelles une partie du protoplasma, colorée en ver't par de la chlorophylle, est dis. posée en rubans spiralés. Deux cellules juxtaposées appartenant à deux filaments voisins présentent chacune, à un moment donné, un renflement vers leur milieu. Les deux petites saillies, remplies par le protoplasma, s'accusant de plus en plus arrivent à se toucher; puis les deux parois cellulaires, qui se sont d'abord soudéesl'une à l'autre, se résorbent; il en résulte un orifice de communication, par lequel le contenu d'une cellule passe dans la cavité de l'autre (fig. 140, I).

La petite masse protoplasmique qui résulte de la fusion des deux corps cellulaires devient une spore ou plutôt, comme on dit, pour marquer son mode de formation, une zygos- 


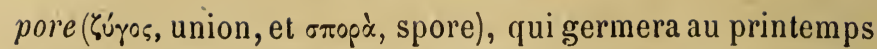
suivant, après avoịr passé l'hiver dans le repos le plus complet (fig. 140, II).

La conjugation s'observe également chez un certain nombre de Champignons les plus inférieurs.

$2^{\circ}$ Fécondation. - La reproduction des Cryptogames par fécondation'se rapproche du mode ordinaire observé chez les Phanérogames. Seulement, les organes reproducleurs ont une forme différente et portent d'autres noms.

Nous allons en indiquer les principaux traits, en prenant d'abord les Algues pour type.

Soit une espèce des plus simples et des plus communes, une Vauchérie(fig.141), qui nous a déjà servi pour la description de la reproduction par rajeunissement. A la surface des cellules d'une de ces Algues filamenteuses on voit se développer une petite saillie recourbée en forme de crochet, la cornicule; c'est l'organe fécondateur ou anthéridie, dont le

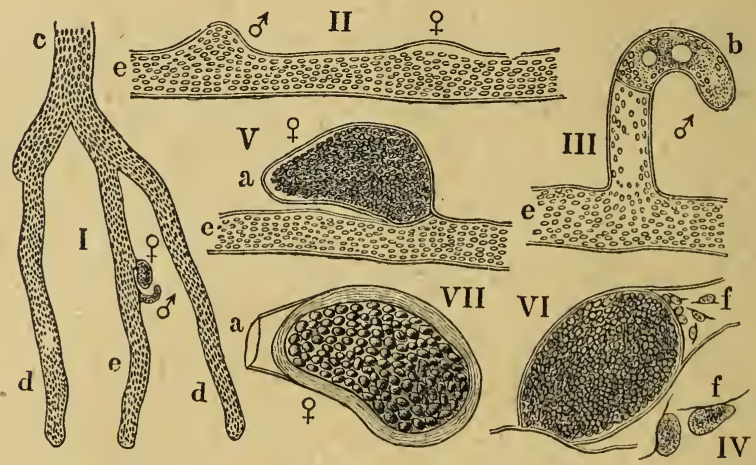

liig. 141. - Marche des phénomènes de la fécondation dans une Algue verte filamenteuse, unicellulaire quoique ramitiée (Vauchérie); I, portion de l'Algue ramifiéc $(c, d, e)$, portant des organes reproducteurs mâle ou cornicule $\left(\sigma^{1}\right)$, et femelle ou sporange (Q), entièrement développes ; II, début de la formation de ces organes; III, la cornicule ou anthéridie entièrement formée et isolée, vers son milieu, du reste du filament par une membrane transversale; IV, les anthérozoïdes $(f)$, auxquels elle a donné naissance; V, le sporange entièrement développé, dont la pointe ou bec (a) s'ouvrira bientôt; VI, le même, dont le bec déchiré laisse passer les anthérozoüdes $(f)$, qui vont féconder son contenu; VII, le sporange fécondé, renfermant de nombreux grains de chlorophylle.

nom rappelle, comme on voit, son analogie avec l'anthère des 
Phanérogames, qui contient le pollen. A côté, sur une autre ccllule, apparaît une saillie de forme différente, ovalaire, le sporange, qui est l'analogue de l'ovaire des Phanérogames. Ces deux productions s'isolent bientôt du reste du filament par suite de la formation, à leur base, d'une cloison de cellulose. Puis, l'extrémité du sporange amincie en forme de bec se ramollit et s'ouvre; il en sort une gouttelette qui laisse un espace vide, dans lequel viennent se précipiter de petits corpuscules très mobiles qui se sont formés dans la cornicule et qu'on appelle des anthérozoïdes

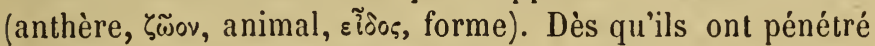
dans le sporange, l'orifice de celui-ci se ferme d'une mombrane de cellulose. Peu après, la spore qui résulte de ces opérations épaissit sa membrane, puis s'isole, et dès lors est susceptible de germer pour devenir une Algue nouvelle.

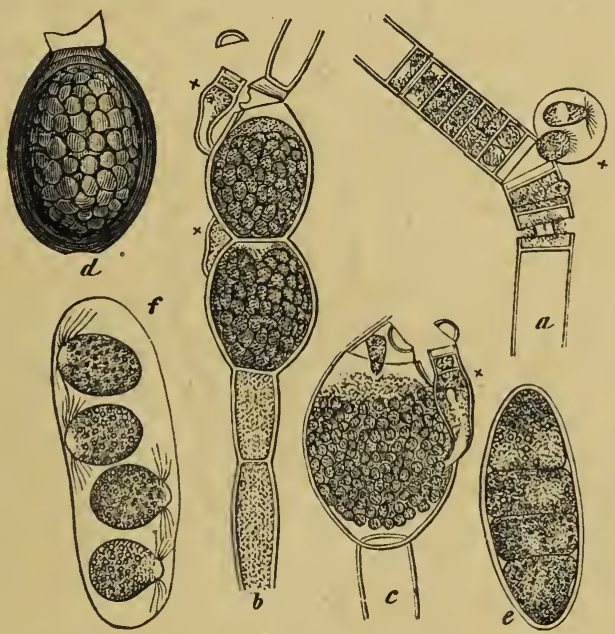

Fig. 142. - Marche de la fécondation dans une Conferve (OEdogonium); a, une portion d'Algue, de laquelle sortent des spores motiles qui deviendront des androspores $(+) ; b$, autre portion portant deux gros sporanges, sur lesquels se sont fixés deux androspores (+), qui vont devenir des anthéridies et fermés par un couvercle qui se lève à un moment donné, comme on le voit sur cette figure et mieux sur la fig. $c$, plus grossie, qui montre en outre un anthérozoïde pénétrant dans le sporange; $d$, la spore fécondée qui s'est aussitôt entourće d'une membrane; $e$, la même, déjà partagée par plusieurs cloisons pour former un nouveau filament d'Algue; $f$, anthérozoïdes.

La fig. 142 nous faiı assister à la marche singulièrement 
compliquée des phénomènes de la reproduction dans une autre espèce d'Algue filamenteuse, commune dans nos fossés, l'OEdogonium ciliatum.

Voici un autre cas : tandis que l'Algue (Vauchérie) dont on vient d'étudier la reproduction est monoïque, les deux sortes d'organes étant portés par le même individu, en voici une autre qui est dioïque, à savoir, le Fucus vesiculosus, qui abonde sur les côles de l'Océan, où il est vulgairement connu sous le nom de Varech (fig. 143).

Sur certains individus on rencontre de petites cavités, les conceptacles (fig. 144), dans lesquelles sont renfermés, au milieu d'un grand nombre de poils ou paraphyses, un certain nombre d'anthéridies. Sur d'autres individus on rencontre également des conceptacles, mais qui ne renferment que des sporanges (fig. 145).

Chaque sporange développe dans son intérieur huit

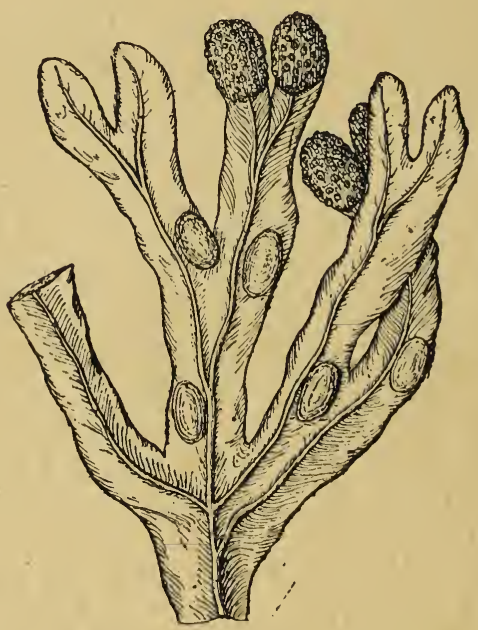

Fig. 143. - Fragment de Fucus vesıculosus, portant des vésicules pleines d'air, et au sommet des frondes des organes reproducteurs.

petites masses ou oosphères, qui, par rupture de leur enveloppe commune, se trouvent mises à nu à un moment donné. 
D'autre part, dans les anthéridies se sont formés des anthérozoïdes qui, au contact de l'eau sont animés de vifs mou-
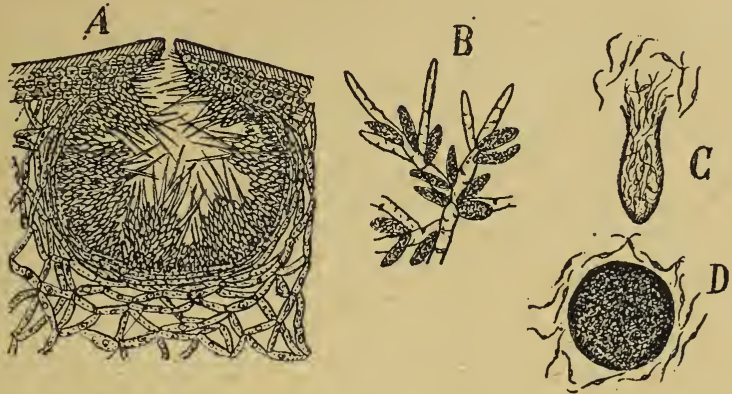

Fig. 144. - Organes fécondateurs du Fucus vesiculosus; $A$, coupe verticale d'un conceptacle renfermant de longs poils portant des anthéridies; $B$, un de ces poils rameux avec nombreuses anthéridies ; $C$, une anthéridie, d'où sortent des anthérozoïdes $; D$, anthérozoïdes fécondant une spore mûre.

veménts, viennent tourbillonner dans le voisinage des premières et les fécondent. Chaque oosphère devient alors une
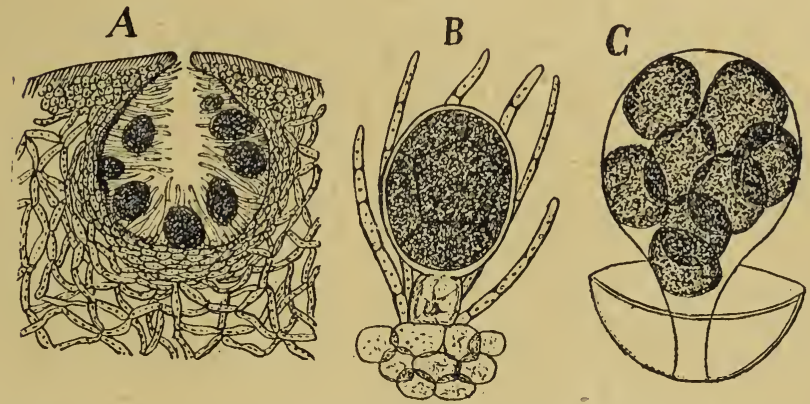

Fig. 145. - Organes femelles du Fucus vesiculosus; $\boldsymbol{A}$, conceptacle renfermant des sporanges portés par de longs poils; $B$, poils portant un sporange; $C$, les huit spores d'un sporange sorti déjà de son enveloppe externe, mais encore renfermé dans son enveloppe interne.

oospore, c'est-à-dire une sorte d'œuf ou de graine, capable de germer et de devenir un individu nouveau. 


\section{FORMES ALTERNANTES CHEZ LES GRYPTOGAMES}

Ce qU'on entend par Formes alternantes. - Un grand nombre de végétaux inférieurs offrent dans leur évolution des phénomènes analogues à ceux que nous présentent un
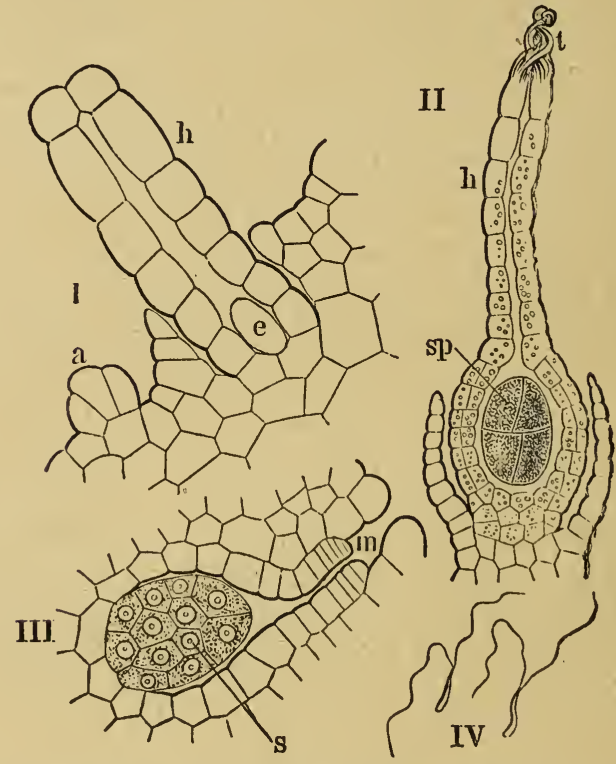

Fig. 146. - Organes reproducteurs des Muscinćes (Hépatiques); I, archégones jeunes de Riccia ciliata, d'après Kny (gross.410 fois); a, apparition d'un arclı́gone ; $h$, col d'un archégone plus développé; $e$, oosphère; II, archégone de Marchantia polymorpha, d'après Sachs (gross. 300 fois); $h$, col de l'archégone; $s p$, l'oosphère, devenue l'oospore par la fécondation et qui commence a se segmenter; $t$, le sommet de l'archégone desséché; III, jeune anthéridie de Riccia glauca, d'après Hofmeister (gross. 500 fois); $s$, cellules-mères des anthérozoïdes; $m$, orifice de l'anthéridie; IV, anthérozoïdes de Marchantia polymorpha, d'aprìs Sachs (gross. 800 fois).

certain nombre d'espèces du règne animal, phénomènes 
désignés sous le nom de formes ou générations alternantes (1).

On entend par là qu'un individu donne naissance à un autre être qui ne lui ressemble nullement et qui, au bout d'un temps plus ou moins long, donnera à son tour naissance à un nouvel être qui ressemblera au premier et non à celui dont il provient directement. Il en résulte, par conséquent, dans le cycle de développement de ces plantes, une alternance de formes souvent très nettement caractérisée. Dans la règle, l'un des termes de la série doit son origine à une génération sexuée, et l'autre à une génération asexuée. La reproduction des Mousses, des Prêles et des Fougères va nous fournir des exemples du premier cas; les Champignons nous en présenteront du second.

Reproduction des Mousses. - Les Mousses, qui possèdent, on l'a vu plus haut, des modes si variés de multiplication, se reproduisent surtout par fécondation (fig. 146). Sur des pieds isolés ou sur un même pied se trouvent des anthéridies ou appareil fécondateur et des archégones (àpyìn, commencement, yòvos, naissance), qui renferment des organes analogues aux ovules. L'anthéridie, à son complet développement, a 'la forme d'un petit corps arrondi ou ovalaire porté sur un court pédicule. Elle est remplie de cellules dont chacune devient un anthérozoïde, sous forme d'un filament enroulé en spirale, avec une extrémité renflée, tandis que l'autre, plus effilée, offre deux longs cils vibratiles. L'archégone a la forme d'une petite bouteille dont la parlie supérieure se prolonge en un long col: Dans la cavité intérieure est une grosse cellule, l'oosphère, surmontée de cellules plus petites, qui se dissociant à un moment donné, permettent aux anthérozoïdes de pénétrer jusqu'à la cellule centrale, laquelle, feécondée, devient l'oospore. Celle-ci, au lieu de fournir directement par sa germination

(1) Voyez le Cours de Zoologie, Anatomie et physiologie animales, p. 129. 
une nouvelle Mousse semblable à celle qui a donné naissance à l'oospore, s'accroît sur place considérablement, sallonge en un filet ou soie (fig. 147, s), dont la croissance force l'archégone à se rompre transversalement vers sa base, de sorte que la soie porte à son sommet la plus grande partie de l'archégone sous forme d'une coiffe $(\mathrm{IV}, m)$, au-dessous de laquelle se trouve ce qu'on appelle le fruit de la Mousse, la capsule $(b)$, où se forment des spores. Celles-ci flottent dans un liquide qui remplit la capsule, sauf sa partie centrale occupée par une petite colonne de tissu solide, la columelle,

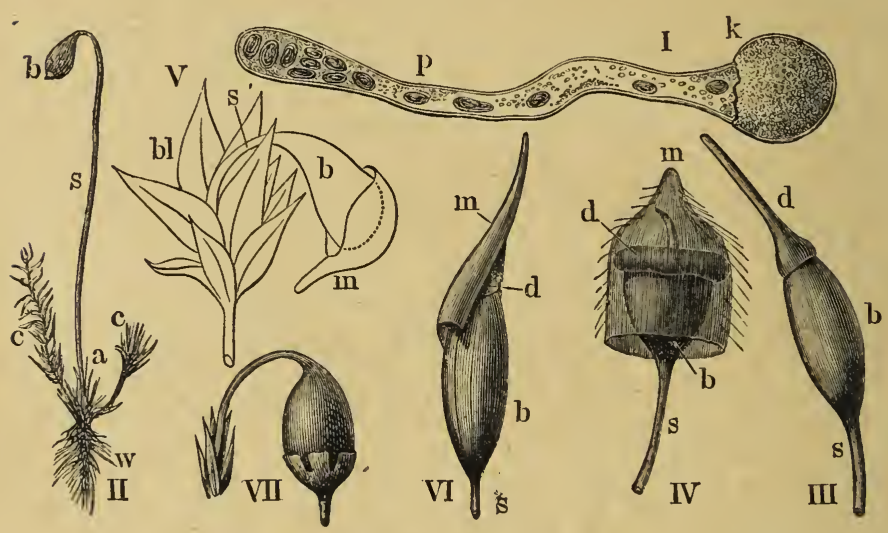

Fig. 147. - Fruit et germination des Mousses; I, spore de Ceratodon purpureus, $(k)$, en voic de germination (gross. 600 fois); $p$, procmbryon; II, tige fructifère de Bryum Warneum (grand. nat.); $w$, rhizoïdes; $c$, ramcaux feuillus ; $s$, soie; $b$, fruit; III à VII, capsules ou fruits de différentes espèces de Mousses (gross. 10 fois) : Ceratodon purpureus, Pogonatum namum, Physcometrium curvisetum, Cynodontium gracilescens, Racomitrium patens; $b$, capsule; $d$, opercule; $m$, coiffe; $b l$, feuilles; $s$, soic.

qui s'épanouit à sa partie supérieure en une sorte de couvercle ou opercule $(d)$, lequel tombe à un moment donné, de sorte que le fruit a la forme d'une urne, à bords ordinairement dentelés et qui laisse sortir les spores ou séminules. La séminule $(\mathbf{I}, k)$, placée sur le sol dans des conditions convenables absorbe l'humidité, se gonfle et germe. Mais la nouvelle production n'est pas encore la plante définitive : c'est un protonema ou proembryon $(p)$, composé de filaments plus 
ou moins nombreux, sur lesquels naîtra enfin la plante définitive sous la forme d'un bourgeon, qui produira des racines par la base, une tige feuillée par le haut (II).

Le cycle de végétation et de reproduction des Mousses est donc singulièrement compliqué. En effet, si nous partons de la spore, nous voyons successivement se développer le prothalle ou proembryon, la plante feuillée ou la Mousse proprement dite, sur laquelle apparaissent ensuite des organes reproducteurs, qui donnent naissance à une oosphère fécondée; enfin vient la génération asexuée des spores, que nous avons prises pour point de départ de tout le cycle du développement.

Reproduction des Prêles ou Equisétacées. - Ces Cryp-

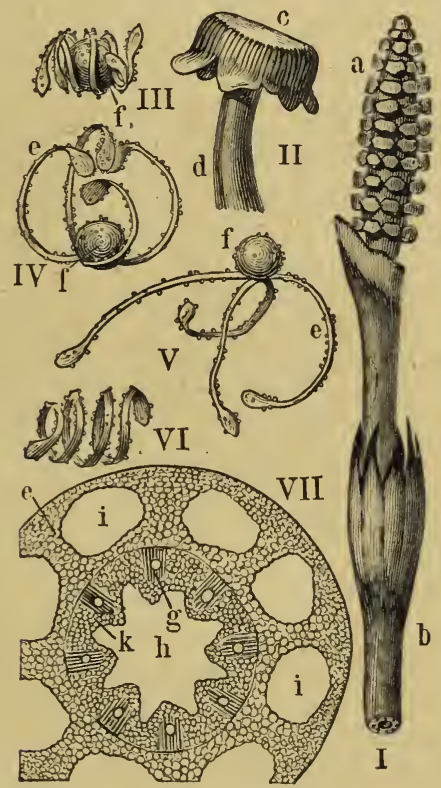

Fig. 148. - Reproduction des Prêles (Equisetum arvense) (grand. nat.); I, rameau fructifère; $a$, les fruits ; $b$, gaîne; Il, un sporange grossi recouvert d'une sorte de bouclier (c), et porté par un pédicule $(d)$; III à VI, spores $(f)$, et leurs ćlatères $(e)$ diversement repliés (gross. 200 fois); VII, coupe transversale de la tige de l'Equisetum limosum (gross. 10 fois); $h$, lacune centrale ; $i, g$, lacunes sur deux rangées alternes entre elles et creusées les unes dans lé cylindre externe ou cortical (lacunes valléculaires), les autres dans le cylindre central (lacunes carénales); $k$, faisceaux tibro-vasculair es. 
togames, à qui leur port tout spécial a valu le nom de Queues de cheval, et qui abondent sur les talus de nos fossés offrent leurs organes reproducteurs disposés en épis à l'extrémité des tiges. On y voit de nombreux sporanges disposés en verticilles serrés et dont chacun offre la forme d'un

clou dont la tête, recouverte d'une

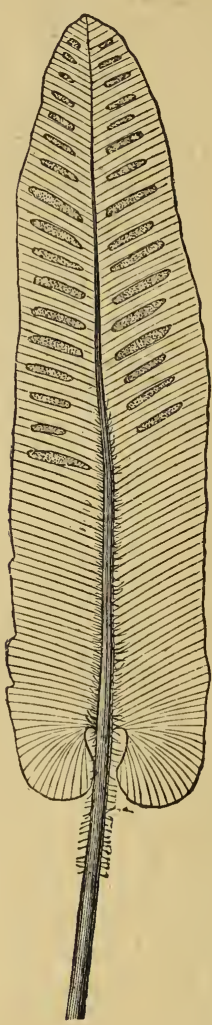

Fig. 149. - Fronde de Fougère (Scolopendrium offi(inarum), avec nombreux sporanges disposés le long des nervures.

plante définitive. sorte de bouclier, serait dirigée en dehors. Les spores ou séminules renfermées dans ces sporanges et qui à un moment donné sont mises en liberté, sont très remarquables; elles sont recouvertes de trois membranes, dont l'externe se divise en quatre petits rubans disposés en croix et qui restent adhérents à la séminule au point de leur entrecroisement. Ces filaments ou élatères sont très hygrométriques et s'ouvrent ou se ferment avec rapidité sous l'influence du moindre changement dans le degré d'humidité de l'air, comme on l'observe facilement au simple contact de l'haleine. La séminule en germant se transforme en un prothalle ou proembryon, en forme de ruban, qui produira des anthéridies et des archégones. Les anthérozoïdes, formés dans les premières, ont la forme d'un ruban spiral portant un paquet de cils vibratiles à une de ses extrémités. Quant aux archégones, ils renferment chacun une oosphère qui, fécondée, devient l'embryon, point de départ de la

En partant de la séminule, nous rencontrons donc successivement un proembryon, sur lequel se forment les 
organes sexuels, anthéridies et archégones, d'où résulte l'embryon, puis la plante définitive qui, par génération asexuée, donne naissance aux séminules.

Il y a donc cette différence avec le cycle évolutif des Mousses, qu'ici c'est le proembryon qui porte les organes mâles et femelles, tandis que dans les Mousses c'est au contraire la plante définitive, c'est-à-dire, l'individu né du proembryon.

Reproduction des Fougères. - A la face inférieure des frondes ou feuilles des Fougères (fig. 149), on remarque souvent, le long des nervures, de petits corps régulièrement disposés. Ce sont des sporanges, qui réunis plusieurs ensemble et souvent recouverts d'une excroissance de l'épiderme de la fronde (indusie), constituent des sores (fig. 150). Dans les sporanges se développent des spores qui, placées sur un sol humide, germent au bout d'un
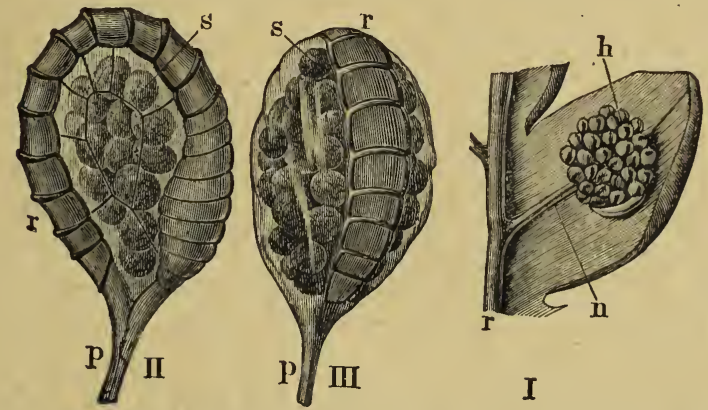

Fig. 150. - Fruits des Fougères; I, foliole d'une fronde de Fougère (Polystichum spinulosum), avec un groupe de sporanges $(h)$, développé sur une nervure secondaire $(n) ; r$, nervure principale (gross. I0 fois); II, un sjorange, vu de face; III, le même, vu de côté; $p$, pédicule; $r$, anneau qui entoure le sporange et qui en se resserrant le déchire pour mettre les spores en liberté; $s$, spores (gross. 200 fois).

temps souvent fort long. Le résultat de la germination ne sera pas la Fougère proprement dite, mais une production intermédiaire, un prothalle ou proembryon, sous forme 
d'une teuille étalée et aplatie (fig. 151), de petite dimension, sur laquelle se formeront, d'une part, des anthéridies $(a)$, d'autre part, des archígones $(b)$. Les premiers produisent

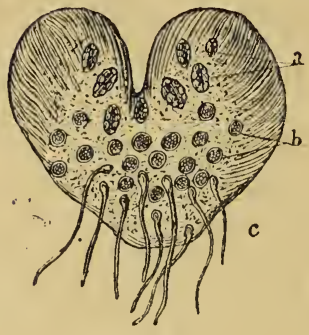

Fig. 151.

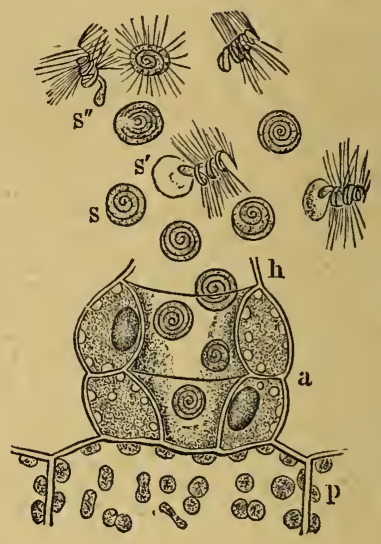

Fig. 152.

Fig. 151. - Prothalle ou proembryon de Fougère; $a$, archégones; $b$, anthéridies; $c$, poils absorbants.

Fig. 152. - Anthéridie de Fougère (Pteris serrulata), dont la paroi (a), a été ouverte, et d'où sont sortis un grand nombre d'anthérozoïdes, dont les uns $(s)$ sont encore enroulés sur eux-mêmes, dont d'autres $\left(s^{\prime}\right)$ sont déroulés, montrent leurs cils vibratiles vers une extrémité et une vésicule à l'autre bout, d'autres enfin ( $s$ ") à leur état définitif, sous forme d'un ruban en spiral.

des anthérozoïdes contournés en tire-bouchon, avec l'extrémité antérieure amincie, pourvue de cils vibratiles, à extrémité postérieure renflée entraînant avec elle une petite vésicule autour de laquelle l'anthérozoïde était naguère enroulé (fig.152). Ces anthérozoïdes pénètrent dans le col des archégones et vont féconder l'oosphère, qui devient l'embryon, lequel formera bientôt, d'un côté, des racines, de l'autre, une tige feuillée, en un mot, la plante définitive.

Il y a donc ici encore alternance de forme ou de génération, puisque nous voyons les spores, développées sans le concours de la fécondation, donner naissance à un proembryon, qui porte à la fois des organes mâles et des organes femelles, de l'union desquels résulte l'oospore qui donne 
naissance à la plante définitive, destinée, comme il vient d'être dit, à porter des spores asexuées.

Reproduction des Rhizocarpées ét Licopodiacées. Les Rhizocarpées, qui sont de toutes petites plantes aquatiques de nos étangs, offrent un cycle de développement analogue à celui des Fougères. On trouve, par exemple, chez les Pilularia, à l'aisselle des feuilles, de petits corps arrondis ou fruits sporifères, renfermant deux sortes de corps reproducteurs : les uns, moins nombreux, et formés de grosses cellules, sont les macrospores ; les autres, bien plus petits et plus nombreux sont les microspores. Celles-ci, en se divisant, forment un prothalle mâle de très faible dimension, qui donnera naissance à des anthérozoïdes sous forme de filaments quatre ou cinq fois enroulés sur eux-mêmes. De même, les inacrospores se développent chacune en un prothalle femelle ou archégone, qui renferme une oosphère, laquelle, fécondée,

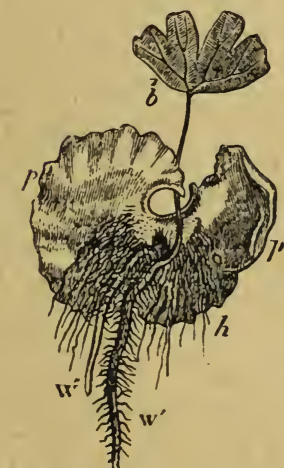

Fig. 153. - Prothalle et plantule de Fougère (Adiantum capillus Veneris); $p$, prothalle; $h$, poils absorbants; $b$, première feuille de la plantule définitive; $w^{\prime}, w^{\prime}$, ses racines.

Champignonsinférieurs, qui vivent en parasites sur d'autres plantes, et dans lesquels la reproduction sexuée ne se montre pas, mais où l'on rencontre seulement plusieurs 
formations successives de spores par voie asexuée. Tantôt, les différentes formes qu'ils présentent dans le cours de leur évolution se produisent sur la même espèce végétale, et tantôt, sur autant d'hôtes d'espèce différente.

Ainsi la famille des Mucédinées est tout entière formée d'espèces qui offrent de semblables particularités. Examinons le développement de l'une d'elles, la Puccinie du Blé (Puccinia graminis); vulgairement appelée la rouille du Blé, en raison de la couleur jaune des taches sous lesquelles elle se montre.

En été, si l'on examine la tige et les feuilles du Blé atteint de cette maladie, on voit, sous leur épiderme, de nombreux filaments serrés, perpendiculaires à leur surface et terminés chacun par une spore ovoïde, formant des bourrelets rougeâtres parallèles aux nervures, et mis à nu par la déchirure de cet épiderme. Les spores, en se détachant, tombent sur les plantes voisines ou sur d'autres parties de la même plante et germent en quelques heures, sous forme de tubes qui pénètrent par les orifices des stomates et se ramifient dans les espaces intercellulaires des tissus. Au bout de quelques jours, apparition de nouvelles spores comme celles qui viennent d'être décrites et répétition des mêmes phénomènes pendant tout l'été, où la rouille orangée, comme l'appellent les cultivateurs, gagne de proche en proche les plantes voisines. A l'automne, au lieu des spores précédentes, il s'en produit d'une forme différente, plus allongées et divisées en deux par une cloison, avec une membrane enveloppante plus épaisse et de couleur brune; c'est la rouille noire des cultivateurs. Ces spores restent inertes pendant tout l'hiver; mais au printemṕs, les deux cellules constituantes de la spore germent et poussent des filaments qui portent bientôt des spores nouvelles ou sporidies, lesquelles enlevées par le vent sont disséminées sur les plantes voisines. Mais il n'y a alors à se développer que celles qui tombent sur une espèce unique, à savoir, l'Épinevinette (Berberis vulgaris). Elles enfoncent des filaments à travers le parenchyme des feuilles; au bout de quelques jours, ces filaments portent des spores de deux sortes, les 
unes, du côté de la face supérieure des feuilles, les autres, du côté de la face inférieure. Or, ces deux sortes de spores ne se comportent pas de la même façon. Les premières, logées dans de petites cavités en forme de bouteille, dont le fond est tout hérissé de poils disposés en un pinceau qui fait saillie à la surface de la feuille, sont, à un moment donné, emportées par le vent; et alors, celles qui tombent dans un milieu convenable germent et produisent à leur tour des sporidies, lesquelles semées sur les feuilles d'Épine-vinette se comporteront comme les précédentes.

Quant à celles de la face inférieure des feuilles, elles sont logées dans des cavités en forme de petites coupes assez larges; disséminées dans l'air, elles ne viennent à germer que si elles tombent sur une feuille ou une tige de Blé. Elles s'allongent en tubes ou filaments, qui s'introduisent par les ouvertures des stomates et s'y développent en un thalle, comme il a été dit au commencement de celte description.

Voilà donc toute une série de formes distincles donnant chacune des spores reproductrices, bien que l'on se trouve en présence d'une seule et même espèce. Ces formes sont tellement différentes, qu'à l'époque où l'on n'avait pas encore suivi toute cette évolution compliquée, on se croyait en présence de genres distincts; ainsi, l'état désigné sous le nom de rouille orangée, qui se voit sur les Graminées pendant l'été, s'appelait Uredo linearis, et celui qui constitue la rouille noire, à l'automne, était le Puccinia graminis, seul nom qui ait été conservé définitivement pour désigner ce singulier végétal protéiforme; les productions en forme de bouteille des feuilles d'Épine-vinette étaient l'Acidiolum exanthematum, et celles en forme de coupe, l'Acidium berberidis.

On pourrait citer bien d'autres espèces du même genre qui subissent des phases de développement analogues; citons-en quelques-unes: le Puccinia straminis, qui constitue une rouille des plus redoutées pour les Graminées et qui passe le printemps sur les Borraginées ; le Puccinia coro- 
nata, qui passe l'été sur l'Avoine, et le printemps sur la Bourdaine et le Nerprun.

D'autres espèces de Puccinies développent, au contraire, leurs quatre appareils reproducteurs successifs sur la même espèce de plantes, par exemple le Puccinia compositarum, sur les Composées ; le Puccinia discoidarum, qui depuis une vingtaine d'années cause en Russie, de grands ravages sur les cultures de Grand-Soleil, dont on retire de l'huile. Enfin, dans certains cas, l'évolution offre une moins grande complication, une, deux ou même trois des phases ordinaires étant supprimées, comme cela se voit, par exemple, pour le Pucciniı prunorum, qui attaque les Pruniers et qui ne produit qu'une sorte de spores, celles d'automne, lesquelles passent tout l'hiver dans l'état de repos; l'on n'observe plus, dans ce cas, qu'une seule forme, qui reste toujours la même.

La famille des Mucédinées renferme d'ailleurs bien d'autres genres dont le développement offre la plus grande analogie avec celui des Puccinies.

Alternance des formes chez les Phanérogames. L'alternance des formes ne se rencontre pas absolument que chez les Cryptogames. Les végétaux les plus élevés présentent des traces non douteuses de semblables phénomènes; mais ce n'est plus, pour ainsi dire, qu'un souvenir, qui même passerait inaperçu si l'on n'y prêtait pas une sérieuse attention. Ge souvenir suffit cependant à établir un pont, une sorte de transition entre les phénomènes du développement de ces deux grands groupes, pourtant si différents du règne végétal, les Cryptogames el les Phanérogames, en passant, comme nous allons le voir, par les Gymnospermes ou Conifères.

Si l'on se rappelle les phénomènes de la reproduction chez ces derniers (Voy. p. 197 et suiv.), on peut se figurer un de leurs grains de pollen comme une spore, l'analogue d'une microspore des Rhizocarpées ou des Lycopodiacíes, qui serait l'aboutissant d'une génération asexuée ou par gemmation, de même que le sac embryonnaire serait une 
autre sorte de spore, l'analogue de la macrospore des Cryptogames qu'on vient de nommer et résultant également d'une génération asexuée.

L'une et l'autre sorte de spores germent: le grain de pollen se divise en plusieurs cellules et forme un prothalle mâle ou anthéridie, dont la cellule terminale émet un tube pollinique, qui au point de vue fonctionnel est l'équivalent de l'anthérozoïde; d'autre part, le sac embryonnaire se développe, avant toute fécondation, en un prothalle femelle, l'endosperme ou albumen, dans lequel se forment les corpuscules, qui jouant le rôle des archégones, renferment l'oosphère, laquelle, à la suite de la fécondation, deviendra l'embryon; celui-ci, en se développant, constituera la plante définitive.

Enfin, chez les Monocotylédones et les Dicotylédones elles-mêmes, le grain de pollen gerne également, mais pour produire simplement le tube pollinique; en est-il de même du sac embryonnaire, qui produit la vésicule embryonnaire; l'un et l'autre sont dus à une génération asexuée. Mais ici, l'anthéridic et l'archégone font défaut; ou du moins, leurs parties essentielles seules existent, à savoir, d'une part, la matière fécondante, renfermée dans le tube pollinique et représentant l'anthérozoïde, d'autre part, la vésicule embryonnaire, représentant l'oosphère et devenant par la fécondation l'oospore ou œuf végétal; celui-ci se développera en embryon, puis en plante définitive, laquelle commencera un nouveau cycle généra teur en produisant, par voie asexuée, comme il vient d'être dit, le pollen et le sac embryonnaire.

\section{PARASITISME}

Faits de Parasitisne chez les Phanérogames. - Un grand nombre de végétaux, parmi les plus inférieurs, vivent en parasites, c'est-à-dire, implantés sur d'autres êtres organisés soit végétaux, soit animaux, aux dépens desquels ils se nourrissent.

Parmi les plantes qui occupent un rang plus élevé, les 
faits de parasitisme sont au contraire bien plus rares, n'ont lieu qu'aux dépens d'autres végétaux et jamais d'animaux. Nous en citerons quelques-uns.

Le Gui (Viscum album), de la famille des Loranthacées, qui se développe sur différentes espèces d'arbres, notamment sur le Pommier, où sa graine a été déposée par quelque oiseau, germe et enfonce sa jeune racine dans l'écorce de l'arbre; arrivée au contact du bois, celle-ci produit des racines secondaires, qui se dirigent en tous sens ontre le bois et l'écorce. D'autres racines enfin, formées sur les précédentes pénètrent, à travers le bois, jusqu'au cœur de l'arbre et sucent la sève destinée à nourrir la plante qui le porte. Mais, comme ce parasite est abondamment pourvu de feuilles vertes, il décompose l'acide carbonique de l'air et y puise une partie des éléments nécessaires à sa nutrition, de sorte qu'il ne fait, en somme, qu'un tort médiocre à la plante sur laquelle il a élu domicile.

La Cuscute (Cuscuta europoea), de la famille des Convolvulacées, qui fait tant de dégâts dans les cultures de Luzerne, de Trèfle et de Chanvre, germe sur le sol, mais enlace cesplantes de sa tige fine et souple. Dans les points où elle se trouve en contact immédiat avec elles, on voit se produire de petites élevures, qui pénètrent jusqu'au centre de la tige hospitalière. Ce sont des racines adventives, qui remplacent la racine primitive, laquelle se détruit de bonne heure. Toutes les racines formées ainsi par la Cuscute sont autant de suçoirs qui pompent les sucs destinés à la croissance de la plante sur laquelle elle a trouvé asile et qui en souffre beaucoup dans son développement, d'autant plus que la Cuscute étant dépourvue d'organes verts, ne peut, comme le Gui, décomposer l'acide carbonique de l'air.

L'Orobanche est de même une plante parasite, de la classe des Personnées, et dépourvue de chlorophylle : elle se développe sur les racines de Luzerne et cause à cette plante fourragère un dommage considérable.

Le Mélampyre, l'Euphraise, le Rhinanthe, tous trois de la famille des Scrofulariacées, vivent également en parasites sur les racines de Graminées, lorsque leurs racines 
secondaires arrivent, en végétant dans le sol, à rencontrer celles de ces plantes, et s'y transforment en suçoirs. Mais comme elles continuent à emprunter au sol la plus grande partie de leur nourriture, elles ne sont que peu nuisibles.

Faits de Parasitisne chez les Cryptogames. - Comme il a élé dit plus haut, le parasitisme, qui est rare chez les Phanérogames, est au contraire extrêmement fréquent chez les Cryptogames. On peut établir ici deux classes de parasites, selon que l'hôte est un végétal ou un animal, et diviser par conséquent les Cryptogames parasites en épiphytes et en épizoïques.

Les Algues renferment parmi leurs espèces les plus petites un très grand nombre de parasites.

Les Champignons surtout, qui étant tous privés de chlorophylle sont incapables de décomposer l'acide carbonique de l'air, doivent emprunter leurs aliments tout formés à des êtres vivants, ou bien végètent sur des débris organiques plus ou moins décomposés, comme les Moisissures, qui se montrent si fréquemment sur nos produits alimentaires liquides ou solides, en sont un exemple vulgaire.

Il a élé question plus haut (p. 224) du singulier parasitisme que nous offrent les Lichens, lesquels résultent, en ṡomme, de l'association d'un Champignon avec une Algue, association qui ne paraît, du reste, être nuisible ni à l'un ni à l'autre.

On a décrit aussi, à l'occasion des formes alternantes des Champignons, le parasitisme de certaines espèces du groupe des Urédinées, bien connues sous le nom de rouille. Il est inutile d'y revenir.

A côté de cette famille se trouve celle des Ustilaginées, qui vivent en parasites sur les Phanérogames. Ainsi, une espèce, la Carie du Blé (Tilletia caries), se développe sur cette Graminée et forme ses spores dans les ovules, qui sont par là même détruits. Le Charbon du Maïs (Ustilago maidis) envahit les ovules et les ovaires du Maïs et les détruit. Le Charbon des céréales n'est autre chose que les Ustilago carbo et destruens, qui attaquent et détruisent la fleur tout entière des Céréales. 
Tous ces Champiguons parasites germent à la surface de leur hôte, enfoncent leurs filaments germinatifs à travers l'épiderme, s'allongent dans les méats intercellulaires, puis produisent leurs spores dans des organes souvent essentiels à la vie de l'individu qui les porte.

On a observé que la Carie du Blé est tuée par le sulfate de cuivre, d'où il suit que pour éviter de semer le parasite avec les grains qui en sont atteints, il suffit de plonger ceux-ci pendant douze à quinze heures dans une solution de sulfate de cuivre à un-demi pour 100 et de semer ensuite.

Le Champignon connu vulgairement sous le nom d'Ö̈dium, qui cause de si grands dégâts dans les vignobles en s'attaquant surtout aux grains des raisins, qu'il enlace de ses filaments, dont il durcit et dessèche l'épiderme et qu'il empêche de s'accroître, est heureusement combattu par la fleur de soufre répandue sur les vignes qu'il a envahies.

La maladie des Pommes de terre, qui parfois se développe avec une telle intensité que des récoltes entières sont détruites, est encore causée par un Champignon, le Péronospora ou plutôt Phytophthora infestans, dont le thalle envoie ses rameaux à travers les tissus des tubercules, s'y ramifie en perçant la paroi des cellules et y envoyant de petits suçoirs.

Un grand nombre d'espèces voisines de la précédente causent des ravages considérables à d'autres plantes, par exemple, le Peronospora viticola, qui détermine dans la vigne la maladie connue sous le nom de mildew (1), le $C y s-$ topus candidus, qui provoque la rouille blanche des Crucifères, el combien d'autres, qui envahissent un grand nombre de nos plantes alimentaires, Laitues, Épinards, Betteraves, Oignons, etc.

La famille des Champignons pyrénomycètes renferme un grand nombre d'espèces parasites des végétaux, plantes herbacées ou ligneuses (Graminées, racines de Luzerne, Trèfle, Betterave, Garotte, Garance, Asperge, Tilleul, Orme, Olivier, etc.). Citons surtout celle, qui à un de ses

(1) Prononcez mildiou. 
états de développement, bien connu sous le nom d'ergot de seigle (Claviceps purpurea) forme sur les épis du Seigle ou des autres céréales ces productions noires que l'on a comparées à un ergot de coq, et qui développées à la place des grains et mêlées à la farine sont très nuisibles à la santé de l'homme.

Les animaux vivants sont fréquemment envahis par des Cryptogames plus ou moins nuisibles. Ainsi, à la base des

Cryptogames épizoïques. dents, surtout chez les personnes qui par un défaut de propreté laissent s'accumuler celte substance calcaire qui porte le nom de tartre dentaire, on trouve une petite Algue, du reste complètement inoffensive, le Leptothrix buccalis (fig. $154, C$ ). Une autre Algue, voisine des Bactéries, mais ayant la forme de petites masses cubiques régulières, la Sarcine, se rencontre fréquemment dans l'estomac de l'Homme et
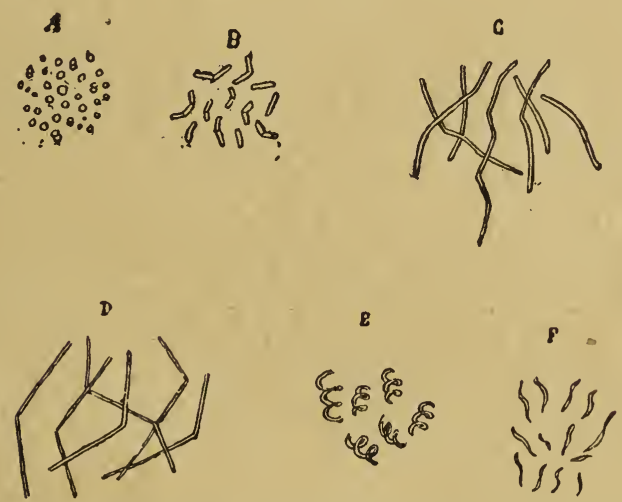

Fig. 154. - A, Micrococcus; B, Bacterium termo; C, Leptothrix buccalis, D, Bacillus anthracis; E, Spirillum; F, Vibrio lineola.

des Mammifères, surtout dans les affections chroniques de cet organe.

Enfin, les infiniment petits qu'on appelle Bactéries ou Microbes, se développent en abondance dans le sang de l'Homme ou des animaux, y pullulent parfois avec une incroyable activité et paraissent être le principe de ces redoutables maladies contagieuses connues sous les noms 
de fièvres pernicieuses, fièvres éruptives, rage, choléra, variole, typhus, charbon, etc.

Un autre Cryptogame, du groupe des Champignons, se rencontre également dans la bouche, mais chez des personnes très affaiblies ou malades, et surtout chez les enfants en bas-âge qui ne reçoivent pas une nourriture convenable. Cette espèce, qui a reçu le noin d'Oidium ou Saccharomyces albicans, caractérise la maladie connue sous le nom de Muguet. Comme elle ne peut se développer que dans un milieu acide, il faut, pour la détruire, employer des lotions alcalines, notamment le borax. Les différentes sortes de teigne sont le résultat du développement d'autres Champignons, Achorion Scheinlenii, Trichophyton tonsurans, etc.; ceux-ci se composent de filaments, qui végètent entre les cellules de l'épiderme, surtout celui de la tête.

D'autres, enfin, attaquent exclusivement les Insectes et notamment plusieurs espèces fort utiles à l'homme, par exemple le Ver à soie, dont la maladie, pour lui si redoutable de la muscardine, est due au Botrytis bassiana.

On a constaté, d'autre part, que des invasions considée rables de Chenilles, qui menaçaient de tout dévorer, ont été arrêtées el réprimées par le développement rapide d'une autre espèce de Champignon, le Cordiceps militaris, de la même famille que celui de l'ergot de seigle, qui les a envahissant, elles et leurs chrysalides, a arrêté leurs ravages.

Comme conclusion à cet aperçu concernant la grave question du Parasitisme, nous dirons que, toutes les fois qu'un animal ou une plante tend à se multiplier outre mesure, de façon à rompre en quelque sorte l'équilibre voulu par la nature, celui-ci tend à se rétablir par la multiplication des ennemis de cet être qui est porté à prédominer, que ces ennemis soient des animaux qui lui demandent leur nourriture, ou des parasites qui, outre la nourriture, y prennent aussi leur domicile. Et c'est là qu'il faut chercher la cause d'une foule de maladies qui sévissent sur nos végétaux les plus utiles, ceux que nous cultivons sur une plus vaste échelle. 


\section{CHAPITRE XIV}

\section{Phénomènes de Mouvement chez les Végétaux.}

Ce qu'il faut entendre par mouvement et sensibilité chez les Végétaux. Mouvements des Végétaux inférieurs. Mouvements de la Racine et de la Tige. Mouvements des Feuilles; Sommeil des feuilles. Mouvements des Fleurs. Influence des anesthésiques sur les mouvements des Plantes.

Ce qu'il faut entendre par Mouvement et Sensibilité CIIEZ LES vÉGÉTAux. - La sensibilité et le mouvement volontaire sont les caractères physiologiques prédominants des Animaux, ceux qui permettent le mieux d'établir une distinction entre eux et les Végétaux; les premiers seuls possèdent d'une façon non douteuse ces propriétés, dites pour cela propriétés animales.

Toutefois, les Végétaux présentent certaines manifestations, qui offrant une réelle analogie avec ces propriétés animales, sont capables de faire illusion. On connaît, en effet, nombre d'organismes végétaux, parmi les plus inférieurs, qui se déplacent comme s'ils étaient mus par une volonté intérieure, leur déplacement semblant dirigé vers un but utile; en outre la plupart des plantes, même de l'organisation la plus parfaite, exécutent dans telle ou telle de leurs parties des mouvements souvent fort remarquables.

Mais dans tous ces cas, il est manifeste que la volonté, la spontanéité fait défaut ; bien loin que ce soit une force intérieure indépendante qui détermine ces mouvements, ce sont toujours les mêmes circonstances extérieures, les mêmes agents, les mêmes excitations qui en provoquent le retour; de sorte que la plante n'est pas libre de les produire ou de s'y soustraire. Qu'il s'agisse, par conséquent, 
de végétaux inférieurs ou de leurs corpuscules reproducteurs que l'on voit nager avec agilité dans un liquide, ou bien d'espèces d'une organisation plus élevée, enfonçant avec persistance leur racine vers le centre de la terre, au lieu de la diriger vers l'atmosphère, l'inclinant vers un endroit humide pour s'écarter d'un point plus aride, etc., dans ces différents cas, il est impossible d'admettre l'exercice d'une volonté et l'existence d'une sensibilité proprement dite, sentie, si l'on peut ainsi parler. Les agents extérieurs, lumière, chaleur, oxygène, etc., agissent sur la substance vivante de ces organismes, sur leur protoplasma, et y déterminent une réaction, d'ailleurs encore assez mal expliquéc, mais qu'assurément on ne peut caractériser du nom de sensation.

Les mouvements exécutés par les végétaux sont de deux ordres, les uns généraux, les autres partiels. Dans le premier cas, la plante se déplace en totalité ; on en rencontre de nombreux exemples dans les espèces les plus inférieures. Dans le second cas, ce sont seulement certaines parties qui subissent des changements dans leur direction, comme un grand nombre de végétaux d'organisation plus parfaite nous en fournissent des exemples. Nous allons ćtudier successivement les uns et les autres.

Mouvements exécutés par les Végétaux infÉrieurs. - Un grand nombre d'or'ganismes inférieurs, que l'on croit devoir ranger dans le règne végétal, auquel ils appartiennent bien, en réalité, par l'ensemble de leurs propriétés, jouissent d'une locomotilité très marquée. Ainsi, sur le tan ou tannée, dont se servent les corroyeurs pour apprêter les cuirs, on rencontre souvent des masses larges de plusieurs décimètres et épaisses de quelques centinìtres, qui se déplacent en rampant et peuvent monter même le long de parois verticales. Ce sont les Myxomycètes, sortes de Champignons muqueux, d'organisation très simple, formés d'un protoplasma très actif, qui émet des espèces de bras ou pseudopodes, servant à fournir des points d'appui à l'organisme, pour permettre à toute la masse de se déplacer. 
MOUVEnents EXÉcutés par la RACINe et la tige 249

Ges infiniment petits, connus sous le nom de Microbes, Bactéries, répandus dans tous les liquides où ont infusé des substances organiques, les milieux oủ se trouvent des substances végétales ou animales en décomposition, sont doués de mouvements variés d'oscillation, d'ondulation ou de rotation sur eux-mêmes, au moyen desquels ils cheminent souvent avec rapidité.

Dans l'eau de nos ruisseaux, de nos mares, se trouvent des Algues filamenteuses, d'un beau vert, formées de cellules placées bout à bout sur une seule file, et qui exécutent des mouvements lents et continus d'oscillation, ce qui leur a valu le nom d'Oscillaires.

Les Diatomées, charmantes Algues microscopiques, revêtues d'une carapace siliceuse et extrêmement abondantes dans nos fossés, exécutent des mouvements singuliers de reptation, au moyen desquels elles se déplacent.

D'autres, les Volvocinées, par exemple, petites Algues de forme arrondie, tourbillonnent avec rapidité dans l'eau.

Les mouvements, enfin, qu'accomplissent nombre de corpuscules reproducteurs d'Algues, sont tellement vifs, paraissent, dans certains cas, si spontanés et sont si manifestement appropriés à un but déterminé, qu'on se croirait en présence d'organismes animaux, d'où le nom de zoospores qu'on leur a donné. Les anthérozoïdes ou corpuscules reproducteurs mâles des Gryptogames exécutent des évolutions non moins intéressantes à étudier.

Ces divers mouvements opérés par les végétaux d'ordre inférieur ou par leurs corpuscules reproducteurs sont difficiles à expliquer. Un certain nombre d'entre eux sont pourvus, il est vrai, de cils vibratiles qui sont des prolongements de la substance vivante intérieure ou protoplasma cellulaire et dont le mouvement détermine le déplacement des petits organismes qui les portent. Mais encore faudrait-il expliquer en vertu de quelle force s'agitent ces prolongements vibratiles. En outre, beaucoup d'espèces n'offrent aucune disposition de ce genre et sont cependant fort agiles.

Mouvements exécutés par la Racine et la Tige. - Un 
des mouvements les plus constants chez les Végétaux est celui en vertu duquel la racine se dirige vers le centre de la terre, et qu'on appelle le géotropisme positif, tandis que la tige s'élève dans l'atmosphère, sous l'influence d'une autre force appelée le géotropisme négatif. On ne sait pas à quoi attribuer sûrement cette direction en sens inverse des deux extrémités de l'axe végétatif. L'action de la pesanteur paraît toutefois y jouer le rôle prédominant, car si on vient à la neutraliser en faisant germer des plantes sur une roue disposée verticalement et mise en mouvement de façon à exécuter environ 150 tours par minute, les racines des différentes plantes en expérience, sous l'influence de la force centrifuge qui se substitue à l'action de la pesanteur, se dirigent toutes vers l'extérieur, toutes les tiges, au contraire, se portant vers le centre de la roue. Si l'expérience a lieu avec une roue tournant horizontalement, la tige se dirige encore vers le centre, mais les racines, obéissant, à la fois, à la force centrifuge et à l'action de la pesanteur qui n'est plus complètement annihilée, se portent encore en dehors, en s'inclinant toutefois vers le centre de la terre, sous un angle d'autant plus près de l'angle droit que la rotation de la roue est moins rapide.

Il existe quelques exceptions à la direction ordinaire des racines : ainsi, celles des Orchidées et des Aroïdées qui, nées à une hauteur variable de la tige, sont flottantes dans l'air, se recourbent souvent vers le haut, au lieu de se diriger constamment en bas; les racines adventives de plusieurs plantes aquatiques amènent leur extrémité jusqu'à la surface de l'eau. De plus, ce qui vient d'être dit ne s'applique qu'au pivot de la racine, et à un degré un peu moindre aux racines de second ordre qui en naissent. Quant aux racines du troisième degré, elles sont complètement soustraites au géotropisme et se dirigent dans le sol suivant toutes les directions.

Un mouvement spécial à certaines tiges seulement, est celui qui consiste dans leur enroulement autour d'un support, ce qui leur a valu le nom de tiges volubiles. La plupart s'enroulent constamment dans le même sens, les unes 
à droite, comme le Haricot, le Liseron, les autres à gauche, comme le Ghèvre-feuille; quelques-unes indifféremment dans un sens ou dans l'autre, par exemple, la Douce-amère. nombre de végétaux demi-ligneux sont pourvus; ce sont comme autant de mains qui se cramponnent, s'enroulent autour des supports voisins et se contournent en spirale pour soutenir la plante, comme on le voit dans la Vigne, la Bryone, les Pois; et, chose remarquable, si les vrilles ne réussissent pas à s'accrocher, elles ne sont plus que des organes inutiles, qui se flètrissent et tombent.

Qu'il s'agisse d'une tige grimpante ou d'une vrille, on observe que le côté qui se trouve en contact immédiat avcc le corps étranger qui sert de support s'accroît moins, tandis que la partie opposée s'allonge davantage, d'où résulte une courbure, la partie interne étant concave et l'externe convexe. Ge phénomène d'accroissement inégal connu sous le nom de nutation est, on le comprend facilement, la cause même qui détermine l'enroulement de l'organe.

Pour se diriger vers la lumière, les différentes parties de Héliotropisme. la tige modifient leur direction. C'est ainsi que les plantes renfermées dans un appartement allongent leurs rameaux et leurs feuilles du côté de la fenêtre. C'est ce qu'on appelle: l'héliotropisme, qui est dit positif, dans les cas où les choses se passent comme il vient d'être indiqué, et négatif, quand les parties de la plante se dirigent en sens opposé, fuyant la lumière, comnie le Lierre en offre un des rares exemples que l'on connaisse.

Mouvements des Feuilles. - Sans insister sur les effets du géotropisme des feuilles, ordinairement positif, en vertu duquel elles se dirigent en haut, et de leur héliotropisme, en vertu duquel elles se portent vers le point le plus vivement ćclairé, nous passerons à ce qu'on a appelé . leur état de veille et leur sommeil.

Dans un grand nombre de plantes, les Légumineuses sommeil des notamment, les feuilles ont, pendant le jour, une direction

Nutation. 


\section{2ङ2 PIĹNOMĖNES DE MOUVEMENT CHEZ LES VÉGÉTAUX}

différente' de celle qu'elles affectent dans la nuit. Dans le premier cas, elles sont épanouies, étalées, tandis que, dans le second, elles se replient les unes sur les autres, se rapprochent des rameaux, qui paraissent alors moins garnis. Les unes se dirigent en haut, comme dans la Luzerne, le Taboc, le Mouron, tandis que les autres s'abaissent, celles du Lupin, de la Surelle, par exemple.

Or, le siège, le point de départ de ces mouvements semble résider dans un renflement que l'on trouve à la base des pétioles et qu'on appelle renflement moteur.

La Sensitive (Mimosa pudica), plante légumineuse très abondante au Brésil, offre l'exemple le plus souvent cité du mouvement des feuilles. Non seulement, en effet, à l'approche de la nuit, les folioles de ses feuilles composéespennées se rabattent les unes sur les autres pour s'appliquer toutes le long des rameaux, de sorte que la plante paraîl flétrie, mais de plus, on peut, par un simple attouchement de ses feuilles, de leurs pétioles et surtout de leurs renflements moteurs, déterminer en elles un effet semblable. Il faut même moins encore: l'ébranlement du sol par le galop d'un cheval, un nuage qui passe, un souffle de l'air suffisent à le produire.

Explication de ces mouvements.

De ce fait que certaines plantes relèvent leurs feuilles, malgré l'action de la pesanteur qui les sollicite en sens inverse, tandis que d'autres les abaissent pendant ce qu'on est convenu d'appeler leur sommeil, il est manifeste qu'il n'y a rien là de comparable au sommeil des animaux. Ce n'est pas un temps de repos; c'est une période pendant laquelle les circonstances extérieures étant différentes, les manifestations vitales se modifient simplement. On va voir, en effet, que bien loin que leurs tissus soient dans un état de : relâchement, de flaccidité, en pareille circonstance, il y a, au contraire, augmentation de leur rigidité, exagération de leur tension. Le mécanisme semble résider entièrement dans la structure du renflement moteur qui, pendant la nuit, devient turgescent, gonflé d'eau, et suivant qu'une structure particulière permet que sa partie supérieure ou sa partie infé- 
rieure se gonfle davantage, car toujours l'une des deux l'emporte sur l'autre, la courbure de la feuille se fait vers le bas, ou au contraile, vers le haut. Quant à la cause même du gonflement du nœud moteur, on l'attribue à ce que la transpiration de la feuille venant à diminuer lorsque la lumière s'affaiblit et disparaît, l'eau qui afflue vers la feuille gorge le pétiole et notamment le renflement en question. De plus, la substance sucrée, la glycose, que la plante a formiée pendant le jour, sous l'influence de la matière verte, s'accumule dans certains organes, notamment dans les nœuds moteurs, pour servir à la nutrition de la plante pendant la nuit. En vertu de son grand pouvoir endosmotique, cette substance attire l'eau en quantité et amène la tension du ressort à son plus haut degré. Cet état est surtout marqué pendant les premières heures de la nuit, puis diminue vers minuit ou une heure du matin, pour disparaître peu à peu à mesure que le jour approche.

Un certain nombre de plantes présentent dans leurs feuilles d'autres mouvements très intéressants sous l'influence d'excitations diverses; telles sont, en particulier, les plantes dites carnivores, comme les Droséra, Dionée gobemouche, etc. (Voy.p. 135 et suivantes.)

Mouvements des Fleurs. - Les différentes parties d'un grand nombre de fleurs exécutent aussi des mouvements. Ainsi, le périanthe en offre assez souvent de périodiques, qui consistent dans son épanouissement à une heure déterminée et son occlusion à heure fixe. Linné a pu établir qu'à chaque heure de la journée, un certain nombre de fleurs s'épanouissaient, et dresser de la sorte l'Horloge de Flore. Ainsi l'ornithogalum umbellatum est vulgairement connu sous le nom de Dame d'onze heures pour la régularité avec laquelle cette plante épanouit ses fleurs à l'heure que son nom indique. Certaines autres, comme le Pissenlit, ne s'épanouissent pas à une heure déterminée d'avance, mais dès le matin, si le temps est beau, dans l'aprèsmidi seulement, si le temps est gris, et restent même complè ement closes, si le ciel est tout à fait sombre.

Périanthe. 
Étanines. Les étamines d'un certain nombre de plantes se penchent vers le pistil à un moment donné, pour y déposer lo pollen (Marronnier d'Inde, Capucine, Fraxinelle, Géranium).

Quelques-unes présentent cetle propriété à un degré poussé fort loin. Ainsi, on voit celles de la Rue s'incliner alternativement une à une et dans un ordre parfait, suivant la place qu'elles occupent, vers le pistil et y rester quelque temps en contact avec lui, pour se relever ensuite et être remplacées par les suivantes.

Les étamines des Berberis et des Mahonia ont une telle irritabilité qu'il suffit de toucher leur filet avec une aiguille, pour les déterminer à se courber aussitòt ver's le pistil et amener le contact de l'anthère avec le stigmate.

Pistil. Enfin, le pistil lui-même, dans son style ou ses stigmates est parfois le siège de mouvements divers qui semblent avoir pour but de le rapprocher des anthères et de favoriser par là même la fécondation. Dans les Nigelles, par exemple, le style se courbe pour que les stigmates puissent venir au contact des élamines situées bien plus bas qu'eux.

Influence des Anesthésiques sur les Mouvements des plantes. - Chose remarquable! plusieurs savants, notamment l'illustre physiologiste Claude Bernard, ont montré que l'espèce de sensibilité ou plutôt d'irritabilité manifestée par un grand nombre de plantes était modifiée, supprimée, comme celle qui est propre aux animaux, par l'action des agents anesthésiques. L'effet de ceux-ci est seulement moins rapide : ainsi, un Oiseau mis sous une cloche où l'on glisse ensuite une éponge imbibée de chloroforme, devient insensible au bout de 4 ou $\boldsymbol{5}$ minutes, tandis que, dans les mêmes conditions, la Sensitive ne perd son irritabilité qu'après un temps cinq fois plus long. On a beau alors toucher la plante dans ses parties naguère les plus sensibles, celles qui réagissaient le mieux au contact des excitants, c'est-à-dire au niveau des renflements moteurs, les feuilles restent étalées au lieu de se rétracter. Si l'on supprime l'action des vapeurs anesthé- 
siques, au bout d'un temps qui se trouve être notablement plus long que pour l'oiseau, notre Mimosa devient de nouveau excitable.

Ces observations qui ont été également faites sur d'autres espèces que la Sensitive (Berbéris, Centaurées, etc.), établissent non pas, il est vrai, une assimilation, mais un rapprochement manifeste entre les deux règnes vivants. Elles trouvent sans doute leur explication dans ce fait que le protoplasna, c'est-à-dire, cette matière que l'on peut appeler la substance vivante, parce que sans elle aucune manifestation vitale n'est possible, le protoplasma, disons-nous, est doué de propriétés essentielles semblables chez l'Animal et chez la Plante, qu'il est contractile, mobile et sensible, ou pour parler plus exactement, irritable, autrement dit, susceptible de réagir sous l'influence d'excitations diverses.

C'est ainsi que se trouve une fois de plus justifiée cette vue profonde que Linné avail portée sur l'ordre magnifique que le Créateur a établi dans ses cuvres, et que le grand naturaliste suédois avait exprimée par ces mots restés célèbres : "Natura non facit saltus" que nous pouvons traduire ainsi : "Tout s'enchaîne dans la nature. " 



\section{TABLE DES MATIÈRES}

Pages.

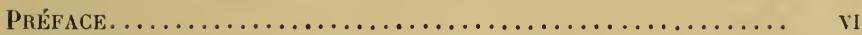

Introduction : Caractères généraux des Végétaux......... xIII

\section{ANATOMIE}

\section{ET PHYSIOLOGIE VÉGÉTALES}

Chap. I. - Éléments anatomiques des Végétaux.

La Cellule est la base de tout organisme végétal. Constitution de la Cellule : Protoplasma, Vacuoles et Courants protoplasmiques; Suc cellulaire ; Noyau et Nucléole. Membrane d'enveloppe; ses modifications de structure : cellules ponctuées, rayées, aréolées, etc. - Fibres. - Vaisseaux. - Laticifères. - Les Tissus des végétaux..................

Chap. II. - Substances diverses contenues dans les cellules végètales.

Classification des produits cellulaires. Amidon; Inuline; Dextrine; Chlorophylle; Substances albuminoïdes cristallisées, Alcurone ; Matières grasses ; Matières sucrées; Tannin; Matières colorantes ; Matières gommeuses ; Concrétions minérales solides; Gaz. - Organes sécréteurs et Secrétions proprement dites : Essences, Résines, etc.; Latex.......... 


\section{ORGANOGRAPHIE VÉGÉTALE}

OU

\section{ANATOMIE DESCRIPTIVE DES ORGANES DES PLANTES}

\section{I - ORganes DE NUTRITION}

\section{Chap. III. - Tige.}

Pages.

Division des fonctions des végétaux. - Division des organes de la nutrition. - Tige. Définition; notions générales sur la Tige. Tiges aériennes : ramification, forme, durée, consistance, port. Tiges souterraines : Rhizome, Bulbe, Tubercule. - Structure de la tige des Dicotylédones : système cortical; zone génératrice ou Cambium; système ligneux ou Bois. Développement et accroissement de la Tige. Tableau résumé de la structure de la Tige. Modifications offertes par la tige de certaines Dicotylédones. - Structure de la tige des Monocotylédones. Modifications offertes par la tige de certaines Monocotylédones. - Structure de la tige des Acotylédones ......................................

\section{Ghap. IV. - Racine.}

Définition. Désignation des racines : $1^{\circ} \mathrm{d}$ 'après le milieu dans lequel elles vivent; $2^{\circ}$ d'après leurs caractères extérieurs. Radicelles. Racines adventives. Durée des racines. Principales modifications : Crampons, Suçoirs, Tubercules. Structure de la Racine. Étude du sommet de la racine: la Pilorhize et le Point végétatif. Allongement de la racine. Étude de la racine à un niveau un peu plus élevé : épiderme ; poils radicaux; écorce, etc. - Différences dans la structure de la racine des Dicotylédones, des Monocotylédones et des Acotylédones. Accroissement de la racine en épaisseur.....

\section{Chap. V. - Feuille.}

Définition et caractères des Feuilles; leur position sur la tige; mode dattache; mode de nervation; forme du limbe et ses modifications. - Structure des Feuilles; développement et croissance; Épiderme et Stomates; Parenchyme foliaire ; Nervures..............................

\section{FONCTIONS DE NUTRITION}

Ghap. VI. - Absorption; Alimentation; Girculation; Transpiration.

Division des fonctions de nutrition. - $A$ BSORPTION. Diffé- 
rentes voles d'absorption: $1^{\circ}$ absorption par les racines; $2^{\circ}$ par les feuilles; $3^{\circ}$ par quelques autres parties. - AliMENTS DES vÉGÉTAUX. Ce qu'on doit entendre par aliments. Origine des principaux aliments des végétaux. - Circulation. Ce qu'il faut entendre par circulation chez les plantes. Ascension de la sève : force d'ascension de la sève; causes de la montće de la sève; composition de la sève ascendante ; voies suivies par la sève ascendante. Sève descendante; voies suivies par la sève descendante. Circulation du protoplasma et du latex. - Trarspiration. Nature de cette fonction. Siège de la transpiration. Activité de la transpiration. Conditions qui modifient cette fonction.........................

\section{Chap. VII. - Suite des fonctions de nutrition.}

RESPIRATION ET FONGTION CHLOROPIYLLIENNE. Différences fondamentales entre ces deux fonctions. Étude comparće de la respiration et de la fonction chlorophyllienne. Conséquences de la respiration : production de chaleur; production de lumière. - Digestion et assimilation. Parties de la plante où s'opère la digestion. Plantes carnivores. Nature des ferments digestifs. - Assimilation. - Réserves nutritives. - Classification des substances assimilées....................

\section{II - Organes et Fonctions de Reproduction}

\section{Ghap. VIII. - La Fleur.}

Composition d'une fleur. - Inflorescences. $-1^{\circ}$ Calice et corolle : description et structure ; 20 Étamines et Pollen: description, structure et développement ; $3^{\circ}$ Pistil ou Gynćcée ; 0vaire, Ovules, Style, Stigmate : description, structure.....

\section{Ghap. IX. - Quelques notions de Morphologie végétale.}

$1^{\circ}$ Origine des parties de la fleur; ce qu'on entend par métamorphoses ascendante et descendante. - Exemples de l'une et de l'autre. - 2० La symétrie dans la fleur. La loi d'alternance. Diagramme et Plan de symétrie de la fleur.........

\section{Chap. X. - Fécondation des Végétaux.}

Pollinisation. Procédés divers au moyen desquels elle s'accomplit. Germination du pollen et fécondation proprement dite. Évolution de l'ovule à partir du moment où il est fécondé. - Fécondation et développement de l'embryon des Gymnospermes. - Hybrides et Métis.................. 
Pages.

\section{Chap. XI. - Fruit et Graine.}

Origine et parties constitutives du fruit. Loges et cloisons. Parties accessoires du fruil. Déhiscence. Classification des fruits. - Constitution et structure de la Graine. Dissémination des fruits et des graines..................

\section{Ghap. XII. - Germination.}

La vie latente de la graine. Conditions nécessaires à la germination. Phénomènes morphologiques et phénomènes chimiques de la germination.........................

\section{Chap. XIII. - Reproduction et Formes alternantes des Cryptogames. - Parasitisme.}

Différents modes de reproduction. Reproduction asexuée par spores; Rajeunissement cellulaire. - Reproduction sexuée : Conjugation; Fécondation. - Formes alternantes chez les Mousses, les Prêles, les Fougères, les Lycopodiacées, les Rhizocarpées et les Champignons. - Alternance de générations chez les Phanérogames. Parasitisme. - Exemples pris parmi les Phanérogames et les Cryptogames; Cryptogames épiphytes et Cryptogames épizoïques................................

\section{Chap. XIV. - Phénomènes de Mouvement chez les} Végétaux.

Ce qu'il faut entendre par mouvement et sensibilité chez les Végétaux. - Mouvements chez les végétaux inférieurs. - Mouvements de la racine et de la tige. - Mouvements des feuilles. Sommeil des feuilles. Mouvements des fleurs. - Influence des anesthésiques sur les mouvements des Plantes..................... 


\section{ERRATA ET ADDENDA}

Page 5, ligne 8, au lieu de : "comparable à celle ", lisez : " comparable à celles $"$. - Même page. Note du bas de la page, au lieu de : "Chap. xiI ", lisez : "Chap. xiv" .

Page 6, ligne 28, par ces mots : "Le suc cellulaire est le milieu....... ", on doit surtout entendre : le suc ou liquide cellulaire qui remplit la cavité de la cellule lorsque le protoplasma en a disparu. C'est ce liquide qui renferme désormais les substances que contenait le protoplasma, notamment l'huile et l'amidon, qui sont mis là en réserve, en attendant leur emploi.

Page 9, au bas de la page. Le ligneux, encore appelé lignine ou vasculose, a pour formule $\mathrm{C}^{19} \mathrm{H}^{12} 0^{10}$ et contient par conséquent plus de carbone et d'hydrogène que la cellulose, dont la formule est $\mathrm{C}^{12} \mathrm{H}^{10} 0^{10}$.

Page 14, ligne 32, au lieu de : "Chap. v ", lisez : "Chap. vi ".

Page 17, par erreur, la fig. 18 représente une espèce d'Algue de la famille des Conjuguées, au lieu d'une Conferve, comme le voudrait le texte. Or, dans les Conjuguées la faculté de se diviser appartient à toutes les cellules du filament, et non pas seuleme̊nt à la cellule terminale. Comme exemple de Conférves, on peut se reporter à la fig. 142, page 227.

Page 20, l'idée exprimée dans la dernière phrase est trop absolue ; beaucoup de parties âgées des végétaux phanérogames et cryptogames vasculaires sont composées de tissu cellulaire.

Page 22, ligne 20, au lieu de : "chlorure de zinc ", lisez : " chloroiodure de zine ».

Page 34, ligne 25, au lieu de : "Chap. vir ", lisez : "Chap. viI ". 
Page 39, ligne 20, au lieu de : "comme c'est le csa ", lisez : "comme c'est le cas $)$.

Page 41, ligne 26, au lieu de: "résine animée ", lisez : "résine animé ).

Page 42, ligne 21, au lieu de : "sont utiliscées à des usages », lisez : " sont employées à des usages ".

Page 56, fig. 43. Des botanistes considèrent les renflements souterrains du Platanthera bifolia, aussi bien que ceux des 0rchidées, comme résultant de l'union intime, de la coalacence de plusieurs racines et par conséquent comme un faux bulbe, plutôt que comme un bulbe plein, lequel s'entend toujours d'une tige modifiée.

Page 70, ligne 34, au lieu de: " n'est autre chose qu'une tige très raccourcie (fig. 54, II); portant des appendices ", lisez : " n'est autre chose qu'une tige très raccourcie (fig. 54, II), portant des appendices ".

Page 99, ligne 8, au lieu de : "les nervures sont, rectinerviées ou curvinerviees parallèles, entre elles ", "lisez: les nervures sont rectinerviées ou curvinerviées, parallèles entre elles ». 






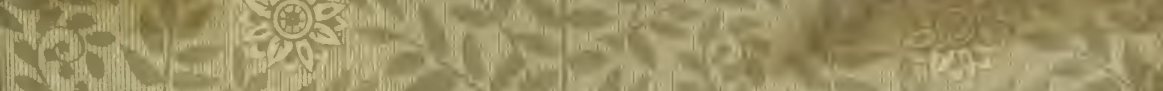

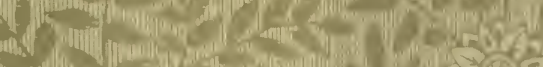

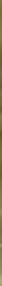

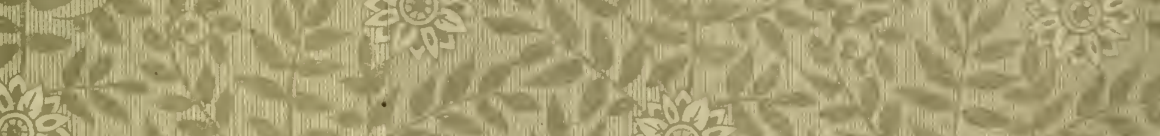

(1)

(3)

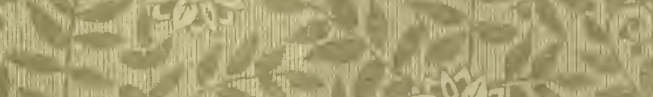

1.

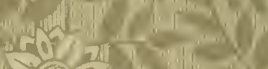

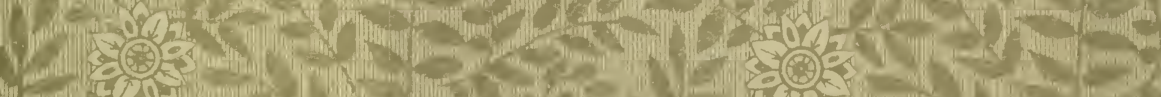

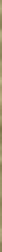
a) 1. if

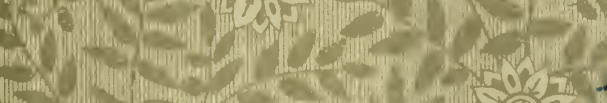

(1)

(i)

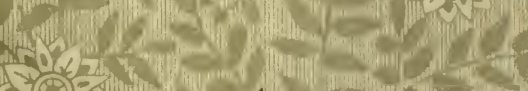
(3) $=3$ int

Tic

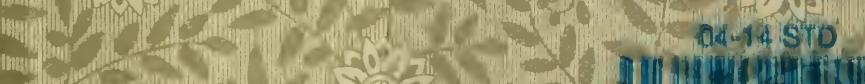

It

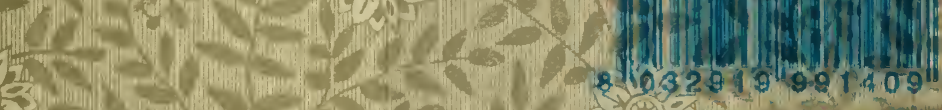

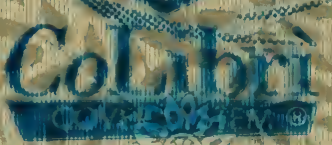

misericing $1501=-13$ 
